\title{
Image Reconstruction in EIT Using Advanced Regularization Frameworks
}

by

\author{
Tao Dai
}

Thesis submitted to the

Faculty of Graduate Studies and Research

In partial fulfillment of the requirements

For the degree of Doctor of Philosophy in

Electrical Engineering

Ottawa-Carleton Institute for Electrical and Computer Engineering

Department of Systems and Computer Engineering

Faculty of Engineering

Carleton University

(C) Tao Dai, Ottawa, Canada, 2008 


$\begin{array}{ll}\begin{array}{l}\text { Library and } \\ \text { Archives Canada }\end{array} & \begin{array}{l}\text { Bibliothèque et } \\ \text { Archives Canada }\end{array} \\ \begin{array}{l}\text { Published Heritage } \\ \text { Branch }\end{array} & \begin{array}{l}\text { Direction du } \\ \text { Patrimoine de l'édition }\end{array} \\ \begin{array}{l}\text { 395 Wellington Street } \\ \text { Ottawa ON K1A 0N4 } \\ \text { Canada }\end{array} & \begin{array}{l}\text { 395, rue Wellington } \\ \text { Ottawa ON K1A 0N4 } \\ \text { Canada }\end{array}\end{array}$

Your file Votre référence ISBN: 978-0-494-43891-6

Our file Notre référence

ISBN: 978-0-494-43891-6

NOTICE:

The author has granted a nonexclusive license allowing Library and Archives Canada to reproduce, publish, archive, preserve, conserve, communicate to the public by telecommunication or on the Internet, loan, distribute and sell theses worldwide, for commercial or noncommercial purposes, in microform, paper, electronic and/or any other formats.

The author retains copyright ownership and moral rights in this thesis. Neither the thesis nor substantial extracts from it may be printed or otherwise reproduced without the author's permission.
AVIS:

L'auteur a accordé une licence non exclusive permettant à la Bibliothèque et Archives Canada de reproduire, publier, archiver, sauvegarder, conserver, transmettre au public par télécommunication ou par l'Internet, prêter, distribuer et vendre des thèses partout dans le monde, à des fins commerciales ou autres, sur support microforme, papier, électronique et/ou autres formats.

L'auteur conserve la propriété du droit d'auteur et des droits moraux qui protège cette thèse. $\mathrm{Ni}$ la thèse ni des extraits substantiels de celle-ci ne doivent être imprimés ou autrement reproduits sans son autorisation.
In compliance with the Canadian Privacy Act some supporting forms may have been removed from this thesis.

While these forms may be included in the document page count, their removal does not represent any loss of content from the thesis.
Conformément à la loi canadienne sur la protection de la vie privée, quelques formulaires secondaires ont été enlevés de cette thèse.

Bien que ces formulaires aient inclus dans la pagination, il n'y aura aucun contenu manquant.

\section{Canada}




\section{Abstract}

Electrical Impedance Tomography (EIT) is an impedance imaging technique which calculates the internal conductivity distribution of a medium from electrical measurements made at a series of electrodes on the medium boundary. EIT is hard because the reconstruction of internal conductivity or conductivity change requires solving a severely ill-conditioned nonlinear inverse problem from noisy data. To solve this, regularization techniques are widely used. EIT is limited by insufficient reliability such as poor spatial resolution and artifacts due to model errors. This is the main obstacle impeding EIT being widely adopted in medical clinical applications. Current research strives to enhance imaging algorithms, robustness against disturbances and reliability of data acquisitions.

The aim of this thesis is to enhance EIT reconstruction performance and robustness using advanced regularization algorithms. This aim is realized through: (1) Determining temporal correlations between images to establish temporal regularization. (2) Exploring $4 \mathrm{D}$ regularization algorithms using both the temporal and a $3 \mathrm{D}$ spatial correlations to improve reconstruction quality. (3) Reconstructing both the conductivity change and the electrode movements from the temporal sequence of EIT measurements to improve both the image quality and the robustness against measurement error. (4) Developing an iterative regularization method in which $\ell_{1}$ and $\ell_{2}$ norm minimizations can be flexibly chosen on the data residue and/or image prior parts. This thesis focuses on addressing the four objectives with results that illustrate enhanced imaging qualities and error/disturbance robustness. Additionally, one variable step size affine projection algorithm and one bioimpedance spectroscopic modeling scheme are developed as related researches of iterative reconstruction and multifrequency EIT. 


\section{Acknowledgements}

The first person I should thank is my research advisor, Andy Adler. For the past three years that I have been working in his lab, it has been his insight, creativity and energy that have been the inspiration for this thesis. I am grateful for the time he spent throughout the whole thesis progress and all publications in my $\mathrm{PhD}$ period. He is also a warmhearted friend who has ensured that my time here in Ottawa was very delightful. By his generous financial support I was able to assist supporting my family and fulfill my study.

I would also like to acknowledge the people who have directly contributed to this work. In particular, professors Manuchehr Soleimani, Behnam Shahrrava, and my colleague Camille Gómez-Laberge, for their kindly encouragements and admirable help on my research works. I would also like to thank my lab colleagues, Camille Gómez-Laberge, Li Peng Xie, Ran Klein, Richard Youmaran, and David Bryan for their friendship. Thanks go also to professors Robert Laganiere, James Green and Yuu Ono for their commitment to improving the quality of this work as members of the thesis committee.

A special thank is to my beloved wife, Yi Lin, for her support and encouragement during these years abroad. Especially the birth of our son, Steven (YiFan) Dai, has made life wonderful and memorable. Also, most sincere thankfulness to my mother, my brother and sister for their unlimited love and support.

Finally, I would dedicate this work to my father, my spiritual support, the most solicitous person to my $\mathrm{PhD}$ convocation but can never see this moment. Because of him, I did not give up even in the hardest time. 


\section{Contents}

Abstract

$\begin{array}{ll}\text { Acknowledgements } & \text { iii }\end{array}$

$\begin{array}{ll}\text { Contents } & \text { iv }\end{array}$

List of Abbreviations $\quad$ viii

List of Symbols $\quad$ X

$\begin{array}{lc}\text { List of Figures } & \text { xii }\end{array}$

List of Tables $\quad$ xiv

1 Introduction $\quad 1$

1.1 Problems . . . . . . . . . . . . . . . . . . 2

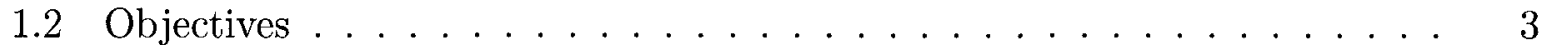

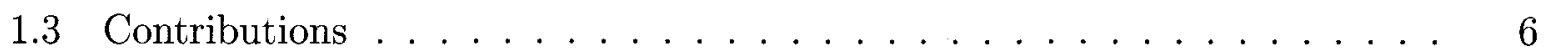

1.3.1 Contributions by Objectives . . . . . . . . . . . 6

1.3.2 Miscellaneous contributions ............... 8

2 Background $\quad 10$

2.1 Bioimpedance Basics . . . . . . . . . . . . . . . . . . . . 10

2.2 Bioimpedance Applications . . . . . . . . . . . . . . . . . . . . 13

2.2 .1 Summary . . . . . . . . . . . . . . . . 13

2.2.2 Bioimpedance method for tissue identification . . . . . . . . . 14

2.2.3 Bioimpedance method for tissue monitoring . . . . . . . . . 16

2.3 Electrical Impedance Tomography . . . . . . . . . . . . . . . . . . . . 17

2.3 .1 History . . . . . . . . . . . . . . . . 17 
2.3.2 Principle of EIT . . . . . . . . . . . . . . . . . . 18

2.3.3 Applications . . . . . . . . . . . . . . . . 23

2.4 Inverse Problem Theory . . . . . . . . . . . . . . . . . 23

2.4 .1 Inverse Problem . . . . . . . . . . . . . . . . . 23

2.4 .2 Inverse Problem on EIT . . . . . . . . . . . . . . . 26

2.4.3 Regularization of EIT . . . . . . . . . . . . . . 27

3 Temporal Image Reconstruction in EIT 32

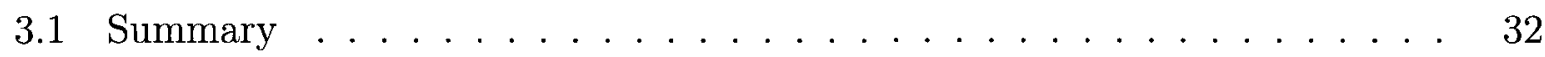

3.2 Introduction . . . . . . . . . . . . . . . . . . 33

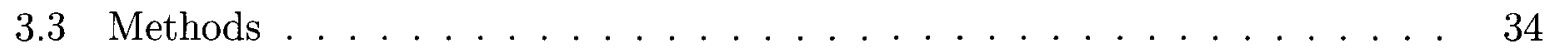

3.3.1 One-step linear GN(Gauss-Newton) solver . . . . . . . . . . . . 35

3.3.2 One-step linear GN solver with weighted data . . . . . . . . . 37

3.3.3 Kalman solver . . . . . . . . . . . . . . . . 37

3.3.4 Temporal one-step solver . . . . . . . . . . . . . . . 38

3.3 .5 Noise figure . . . . . . . . . . . . . . . . . . . . . 40

3.4 Results . . . . . . . . . . . . . . . . . . 42

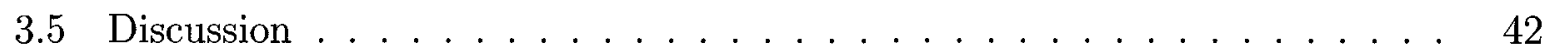

4 EIT Image Reconstruction with 4-D Regularization $\quad 47$

4.1 Summary . . . . . . . . . . . . . . . . . . . . 47

4.2 Introduction . . . . . . . . . . . . . . . . . 48

4.3 Methods ......................... 50

4.3.1 One-step linear Gauss-Newton solver . . . . . . . . . . . . . 51

4.3 .2 Temporal solver . . . . . . . . . . . . . . . . 52

4.3.3 3-D spatial prior with full model correlation . . . . . . . . . . 54

4.3.4 Methods: Simulations . . . . . . . . . . . . . 57

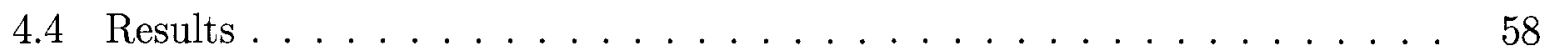

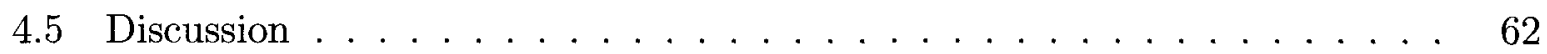

5 Temporal Reconstruction of Conductivity and Electrode Movement $\quad 66$

5.1 Summary ............................. 66

5.2 Introduction . . . . . . . . . . . . . . . . 67

5.3 Methods . . . . . . . . . . . . . . . . . . 68

5.3.1 One-step linear Gauss-Newton (GN) solver . . . . . . . . . . . . . 69

5.3.2 Reconstruction of conductivity change and electrode movement . . 71 
5.3.3 Temporal one-step solver . . . . . . . . . . . . . . . . . . 72

5.3.4 Temporal reconstruction of conductivity and electrode movement . 73

5.3 .5 Method: experiments . . . . . . . . . . . . . . 73

5.4 Results . . . . . . . . . . . . . . . . . . . 74

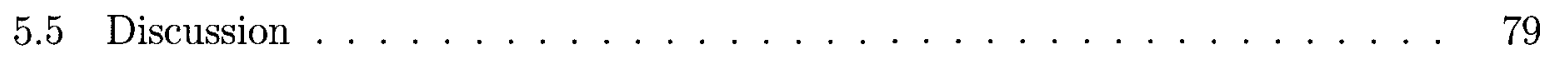

6 Iterative $\ell_{1}$ Norm Regularization of EIT $\quad 81$

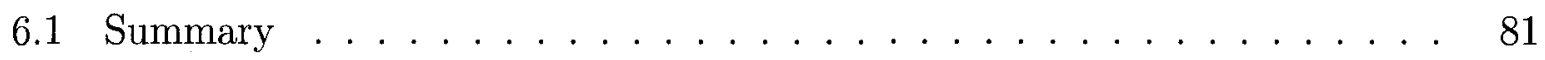

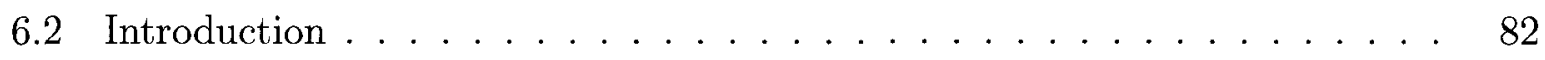

6.3 Methods . . . . . . . . . . . . . . . . . . 83

6.3 .1 Least Squares $\left(\ell_{2}\right.$ norm) solution $\ldots \ldots \ldots \ldots \ldots$

$6.3 .2 \ell_{1}$ norm solution $\ldots \ldots \ldots \ldots \ldots \ldots \ldots$

6.3.3 Generalized $\ell_{1}$ and $\ell_{2}$ regularization with iterative method . . . . 85

6.4 Simulation . . . . . . . . . . . . . . . . . . 86

6.5 Results . . . . . . . . . . . . . . . . . . . . . 88

6.6 Discussion . . . . . . . . . . . . . . . . . . . . 89

$\begin{array}{lll}7 & \text { In Vivo Blood Characterization from BIS } & 90\end{array}$

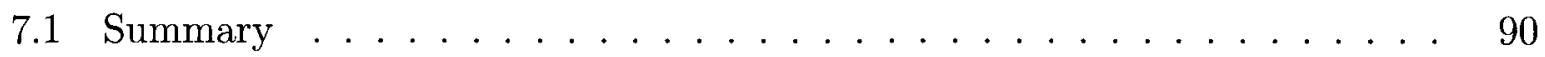

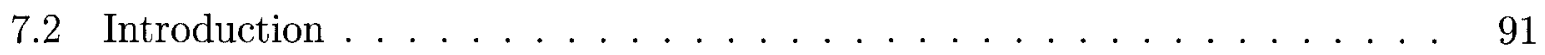

7.3 Methods . . . . . . . . . . . . . . . . . . . . . 92

7.4 Methods: experimental . . . . . . . . . . . . . . . 95

7.4 .1 Experimental Phantom . . . . . . . . . . . . . . . 95

7.4.2 Equivalent Circuit Modeling and Simulation . . . . . . . . . 96

7.5 Results . . . . . . . . . . . . . . . . . . 97

7.5 .1 Experimental Phantom . . . . . . . . . . . . . . 97

7.5.2 Equivalent Circuit Modeling and Simulation . . . . . . . . . 98

7.6 Discussion . . . . . . . . . . . . . . . . . . . . . . . 101

8 Conclusion and Future Work $\quad 106$

8.1 Conclusions . . . . . . . . . . . . . . . . . 106

8.2 Future work . . . . . . . . . . . . . . . . . . . . . . . 107

\section{Appendices}


A VS-APA with a Weighted and Regularized Projection Matrix

A.1 Summary . . . . . . . . . . . . . . . . . . . . 110

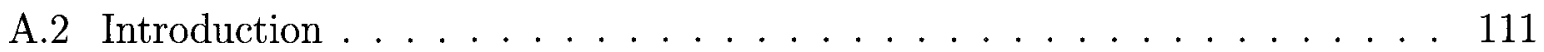

A.3 Methods . . . . . . . . . . . . . . . . . . . . . . 112

A.3.1 Optimal Variable Step-Size APA _. . . . . . . . . . . . 112

A.3.2 Optimal Variable Step Size APA with Forgetting Factor . . . . . 113

A.4 Algorithm Stability Analysis . . . . . . . . . . . . . . . . 115

A.5 Regularization of the Weighted Projection . . . . . . . . . . . 117

A.6 Simulation Results . . . . . . . . . . . . . . . . . . 118

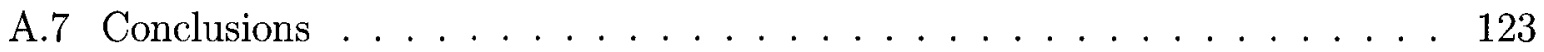

B Formulation of $\ell_{1}$ Norms Regularization $\quad 126$

B.1 Exponent on D . . . . . . . . . . . . . . . . . . . . . . 126

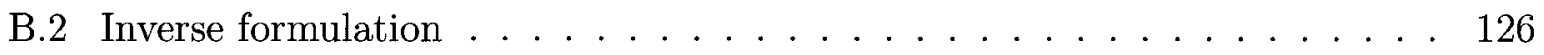

B.3 Data formulation for inverse . . . . . . . . . . . . . 127

$\begin{array}{lr}\text { Bibliography } & 142\end{array}$ 


\section{List of Abbreviations}

\begin{tabular}{|c|l|}
\hline Abbreviation & \multicolumn{1}{|c|}{ Details } \\
\hline APA & Affine Projection Algorithm \\
BEM & Boundary Element Method \\
BIA & Bioelectrical Impedance Analysis \\
BIS & Bioelectrical Impedance Spectroscopy \\
CT & Computed Tomography \\
ECF & Extracellular Fluid \\
ECFC & Extended Cole-Fricke-Cole \\
ECG & Electrocardiogram \\
EIDORS & Electrical Impedance and Diffuse Optics Reconstruction Software \\
EIT & Electrical Impedance Tomography \\
EM & Electrode Movement \\
FAP & Fast Affine Projection \\
FEM & Finite Element Model \\
GN & Gauss-Newton \\
ICF & Intracellular Fluid \\
IRLS & Iteratively Reweighted Least Squares \\
LM & Levenberg-Marquardt \\
LMS & Least Mean Squares \\
LS & Least Squares \\
MER & Maximum Entropy Regularization \\
MIT & Magnetic Induction Tomography \\
MFBIA & Multi-Frequency Bioelectrical Impedance Analysis \\
MFEIT & Multi-Frequency Electrical Impedance Tomography \\
\hline
\end{tabular}




\begin{tabular}{|c|l|}
\hline Abbreviation & \multicolumn{1}{|c|}{ Details } \\
\hline MRI & Magnetic Resonance Imaging \\
MSE & Mean Squared Error \\
NF & Noise Figure \\
NLMS & Normalized Least Mean Squares \\
NOSER & Newton's One-Step Error Reconstructor \\
NSR & Noise Signal Ratio \\
PD-IPM & Primal Dual Interior Point Methods \\
RLS & Recursive Least Squares \\
SNR & Signal Noise Ratio \\
SVD & Singular Value Decomposition \\
TBW & Total Body Water \\
TV & Total Variation \\
VS-APA & Variable Step-Size Affine Projection Algorithm \\
VS-APA-FF & Variable Step-Size Affine Projection Algorithm with Forgetting \\
& Factor \\
VS-APA-FF-REGU & Regularized Variable Step-Size Affine Projection Algorithm with \\
\hline
\end{tabular}




\section{List of Primary Symbols}

Symbols are globally defined as below, except otherwise defined at their presence as different indications. The general notation of the thesis: matrices are in bold and uppercase; vectors are in bold and lowercase; scalars are in lowercase; the $(i, j)^{t h}$ entry of a matrix A is $A_{i j}$; the $i^{\text {th }}$ entry of a vector $\mathbf{x}$ is $x_{i}$.

\begin{tabular}{|c|l|}
\hline Symbols & \multicolumn{1}{|c|}{ Description } \\
\hline $\mathbf{A}$ & state transition matrix, or diagonal matrix of volume/area \\
$\mathbf{B}$ & Cole distribution constant \\
$\mathbf{C}$ & linearized one-step reconstruction matrix \\
$d$ & error covariance matrix \\
$\mathbf{d}$ & half width of temporal window, or desired signal \\
$\delta$ & vector of desired signal \\
$\mathbf{e}$ & inter-frame delay \\
$\varepsilon$ & dielectric permittivity \\
$\varepsilon_{r}$ & relative permittivity \\
$\eta$ & spatial correlation constant \\
$f_{c}$ & characteristic frequency \\
$\gamma$ & temporal correlation constant, or exponential decay factor \\
$\mathbf{\Gamma}$ & temporal weight matrix \\
$\mathbf{I}$ & identity matrix \\
$J$ & current density \\
$\mathbf{J}$ & Jacobian matrix \\
$\mathbf{K}$ & Kalman Gain \\
$\lambda$ & regularization parameter, or hyperparameter \\
$\mu$ & step size \\
$\mathbf{n}$ & noise sequence \\
$n_{E}$ & number of electrodes \\
\hline
\end{tabular}




\begin{tabular}{|c|l|}
\hline Symbols & \\
\hline$n_{M}$ & number of data points \\
$n_{V}$ & number of effective measurements from a single stimulation \\
$\Omega$ & medium body under investigated \\
$\phi$ & potential \\
$\mathbf{p}$ & projected error vector \\
$\mathbf{P}$ & inverse of $\mathbf{R}$ \\
$p_{n}$ & norm type value of data residue \\
$p_{x}$ & norm type value of image prior \\
$\mathbf{r}$ & a 3D point vector \\
$R_{0}$ & impedance at very low frequency \\
$R_{\infty}$ & impedance at very high frequency \\
$\mathbf{R}$ & prior matrix, inverse of normalized $\boldsymbol{\Sigma}_{x}$ \\
$\sigma$ & conductivity, or standard deviation \\
$\sigma_{n}$ & average amplitude of channel noise \\
$\sigma_{x}$ & a priori amplitude of conductivity change \\
$\boldsymbol{\Sigma}_{\mathbf{n}}$ & covariance matrix of the measurement noise $\mathbf{n}$ \\
$\boldsymbol{\Sigma}_{\mathbf{X}}$ & covariance matrix of the desired image $\mathbf{x}$ \\
$\boldsymbol{\Sigma}_{\mathbf{y}}$ & covariance matrix of the data $\mathbf{y}$ \\
$t$ & time index \\
$t_{F}$ & acquisition time of one single frame \\
$U$ & input matrix \\
$\mathbf{v}$ & measurement vector \\
$\mathbf{V}$ & inverse of $\mathbf{W}$ \\
$\mathbf{w}$ & coefficent/weight vector of the linear adaptive filter \\
$\mathbf{W}$ & weight matrix for data residue, inverse of normalized $\mathbf{\Sigma}_{n}$ \\
$\mathbf{x}$ & image vector \\
$\mathbf{x}$ & desired conductivity changes \\
$\mathbf{y}$ & data vector \\
& impedance \\
\hline
\end{tabular}




\section{List of Figures}

1.1 Image sequence with temporal correlation $\ldots \ldots \ldots \ldots$

1.2 Four dimensional correlation model . . . . . . . . . . . . . . . . 4

1.3 reconstruction of both electrode movements and conductivity changes . . . 5

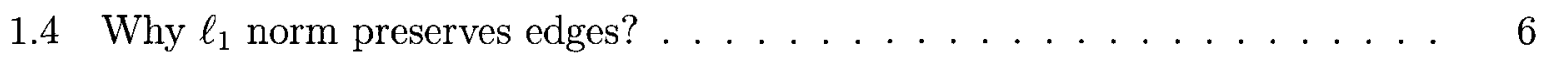

2.1 A piece of cylindric material to illustrate bioelectrical properties . . . . . 11

2.2 dielectric dispersion of biomaterials $\ldots \ldots \ldots \ldots \ldots \ldots$

2.3 Typical Cole-Cole curves of some tissues. . . . . . . . . . . . . . . 15

2.4 A block diagram of EIT system _ . . . . . . . . . . . . . . . . . . 19

2.5 A typical difference EIT image: Lung ventilation image sequence . . . . . 21

2.6 Principle of CT image reconstruction by back-projection . . . . . . . 28

2.7 Principle of EIT image reconstruction by back-projection . . . . . . . . . 28

2.8 Iterative reconstruction of EIT . . . . . . . . . . . . . 30

3.1 Algorithms are compared using a high hyperparameter . . . . . . . . . . 43

3.2 Algorithms are compared using a low hyperparameter . . . . . . . . . . 44

4.1 The 3-D forward model . . . . . . . . . . . . . . . . . . . . . 59

4.2 The trajectory of the target in the forward model . . . . . . . . . 60

4.3 Comparison of algorithms with no noise . . . . . . . . . . . . 61

4.4 Comparison of algorithms with noise $\ldots \ldots \ldots \ldots \ldots$

$4.5 \gamma$ as a function of target speed and noise level $\ldots \ldots \ldots \ldots$

5.1 2D numerical model simulation and saline phantom measurement $\ldots \ldots 75$

5.2 Effect of boundary distortion on EIT reconstruction . . . . . . . . 76

5.3 Images reconstructed from simulated data . . . . . . . . . . . . 77

5.4 Images reconstructed from measured data of a saline phantom $\ldots . . .78$

5.5 Pulmonary monitoring of a human subject (maximum expiration) $\ldots . .79$ 
6.1 Forward FEM . . . . . . . . . . . . . . . . . . . . . . . . 87

6.2 Image reconstruction with different $\ell_{1}$ and $\ell_{2}$ norms $\ldots \ldots \ldots 88$

6.3 Images reconstructed with different data norms (electrode error added) . . 89

7.1 A tetrapolar BIS sensor applied to a forearm model . . . . . . . . . . . 92

7.2 The forearm measured is modelled as three components in parallel . . . . . 93

7.3 Cole-Cole plot in the complex impedance plane . . . . . . . . . . . . 94

7.4 Tissue phantom with a tetrapolar BIS sensor applied . . . . . . . . . 95

7.5 The equivalent circuit of a cylindric biomaterial compartment _ . . . . . 97

7.6 Bioimpedance spectroscopy modelling using Cole-Cole curves . . . . . . . . 99

7.7 Blood impedance curve is fitted using Cole-Cole model (noisefree) $\ldots . . .100$

7.8 Blood impedance curve is fitted using Cole-Cole model $(n s r=0.05) \ldots 101$

7.9 Estimation error with respect to noise levels . . . . . . . . . . . . . 102

7.10 A Preliminary Blood Pooling Experiment on Human Arm . . . . . . . . . 103

$7.11 \ldots \ldots \ldots \ldots \ldots \ldots$. . . . . . . . . . . . . . 104

A.1 System identification model . . . . . . . . . . . . . . . . . . . . . . . . . . 119

A.2 Comparison between VS-APA-FF and VS-APA, G2 colorization . . . . . 120

A.3 Comparison between VS-APA-FF and VS-APA, G1 colorization . . . . . 121

A.4 Algorithms with small $\lambda$, G2 colorization . . . . . . . . . . . . 122

A.5 Algorithms with small $\lambda$, G1 colorization . . . . . . . . . . . 123

A.6 Algorithms with large $\lambda, \mathrm{G} 2$ colorization . . . . . . . . . . . . . . 124

A.7 Algorithms with large $\lambda$, G1 colorization . . . . . . . . . . . 125 


\section{List of Tables}

2.1 Typical Cole-Cole parameters of some tissues. . . . . . . . . . . . . . 14

4.1 planar positions of reconstructed target $\ldots \ldots \ldots \ldots$

7.1 Estimated $(\hat{m})$ v.s Original $\left(m_{0}\right)$ Cole-Cole Parameters $\ldots \ldots \ldots$ 


\section{Chapter 1}

\section{Introduction}

This chapter provides a brief introduction to the field of study with the goal of clarifying the problems to be addressed, and the contributions of this thesis. The background material is reviewed in more detail in chapter 2 , where detailed references to the literature are provided.

Electrical impedance tomography (EIT) is a non-invasive imaging modality that can be used to image conductive subjects. In EIT the internal conductivity distribution of the subject is reconstructed based on electrical measurements from electrodes attached around the boundary. In medical applications, due to the differences in bioelectrical properties between tissues, the conductivity distribution can show the structural and functional properties of the subject. For example, different organs of human body show a contrast in bioimpedance imaging. Further, physiological variations, such as increased blood volume in lungs, cause bioelectrical property changes which can be imaged as a varying conductivity distribution.

In EIT, electrodes are attached on the surface of a subject and a certain current pattern is injected into the subject through stimulation electrodes. Normally alternating current is used as stimulation whose amplitude is usually several $\mathrm{mA}$ with frequency between $1 \sim 100 \mathrm{kHz}$. The voltages are measured using voltage measurement electrodes. An image reconstruction method is then used to calculate the internal conductivity distribution from the boundary data.

EIT equipment has relatively low cost and good portability and it is easy to be operated and maintained. The current stimulation is not hazardous to humans in contrast to 
exposure to x-ray or radioisotopes in nuclear medicine. The data acquisition speed is very high so that it can capture fast-varying physiological activities. EIT shows potential to be used as a bedside realtime monitoring system which is affordable in clinics.

\subsection{Problems}

The reconstruction problem of EIT is to recover an unknown impedance distribution from boundary data. This process is severely ill-conditioned. In order to solve an ill-conditioned problem, certain special methods are required. A regularization technique is a process which can stabilize the inversion process by imposing additional constraints from a priori knowledge of the true solution.

The spatial resolution in EIT is relatively poor compared with other modalities, such as Magnetic Resonance Imaging (MRI) and Computed Tomography (CT). However, EIT has very good temporal resolution. The fastest EIT system can provide as many as 1000 images per second. Thus EIT is an excellent imaging modality to perform real-time monitoring on fast-varying physiological activities, e.g., cardiopulmonary status. Adjacent EIT images, as well as data frames, are highly correlated. This temporal correlation provides additional information content to that from a single frame. This information can be used to increase the quality of the image reconstruction. Currently most EIT reconstruction algorithms solve data frames independently, although iterative methods, e.g., Kalman filter based algorithms, reconstruct images based on current and previous frames. This thesis proposes the first one-step linear reconstruction algorithm that utilizes temporal correlations formulated in terms of the regularization prior.

Besides the ill-conditionness of EIT, reconstruction quality of EIT is highly sensitive to model errors, e.g., incapability of modeling the real subject perfectly. For example, the shape of the reconstruction model is more or less different from the real subject being measured. Another example is the medium deformation involved during in vivo measurement, e.g., thoracic cage movement due to breathing. Electrode uncertainty is another significant degradation of EIT performance. For example, electrode movement, variable electrode contact impedance, and drift in the electronics can produce dramatic artefacts in images. Other than hardware improvements, reconstruction algorithms need to be upgraded to compensate model errors and be robust against electrode uncertainty. This is 
a prerequisite to bring EIT, a promising medical imaging modality, into routine clinical applications.

\subsection{Objectives}

The thesis investigates novel reconstruction algorithms in order to improve EIT imaging performance in terms of image quality (spatial resolution and noise level) and robustness. This aim is addressed in terms of:

1. Investigating the inter-frame correlation of adjacent data frames and determining the inter-image correlation and use it to improve EIT image performance. The temporal correlation of images is illustrated as Figure 1.1.

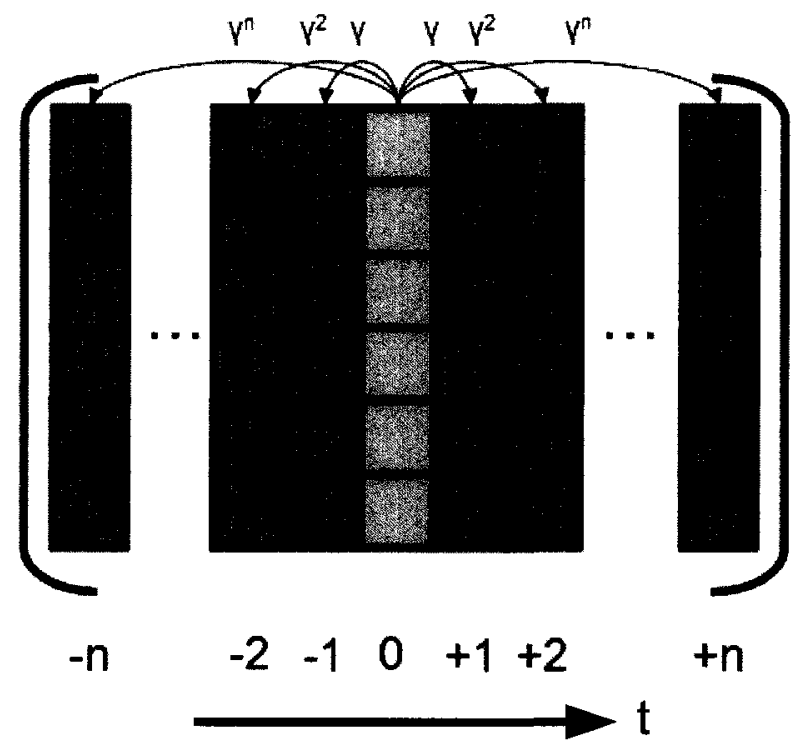

Figure 1.1: Image sequence with temporal correlation. One image vector is described as a block column. The current image is at $t=0 . \gamma^{k}$ is the inter-image correlation between two images with delay/leading of $k$, where $\gamma$ is temporal correlation constant.

2. Developing a general approach to model the 4D (temporal and 3D spatial) correlations in the regularization prior of a single step Gauss-Newton type linearized reconstruction. The 4D correlation model is illustrated as Figure 1.2. 


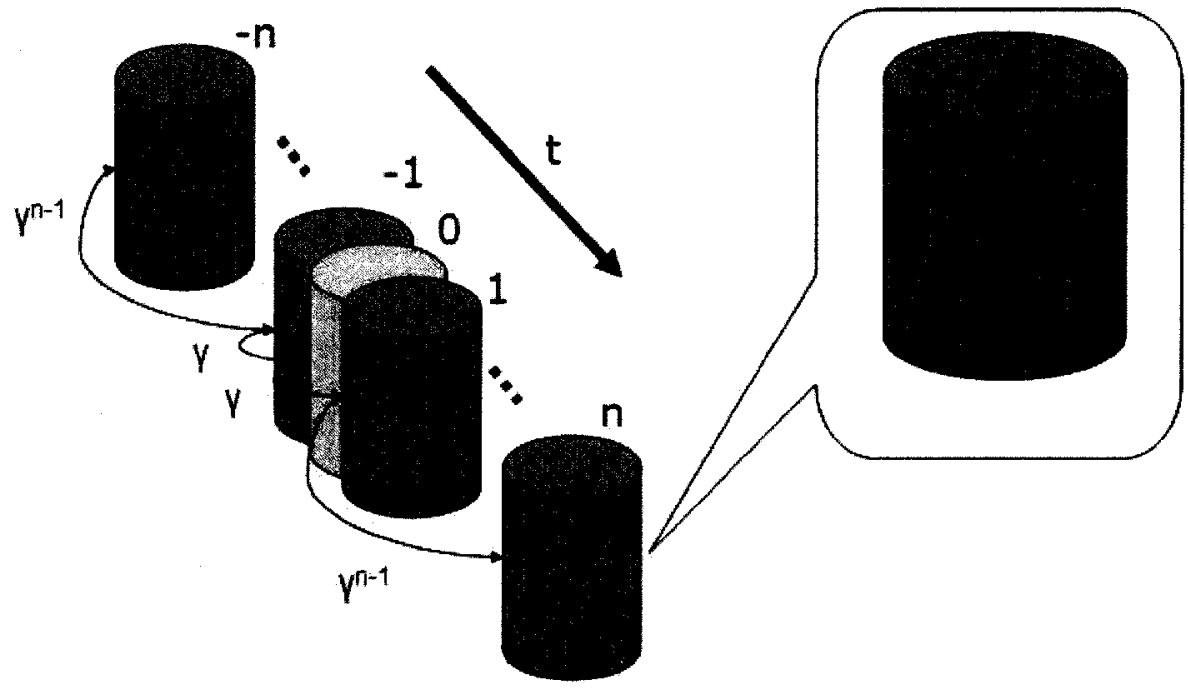

Figure 1.2: Three dimensional image sequence with temporal correlations. Three dimensional image is described as a cylindric subject. The current image is at $t=0 . \gamma^{k}$ is the inter-image correlation between two images with delay/leading of $k$. Two arbitrary elements (marked as red circles) within a $3 \mathrm{D}$ image has spatial correlation $\eta^{d}$, where $\eta$ is spatial correlation constant and $d$ is distance between any two elements.

3. Investigating the applicability of temporal regularization techniques for enhancing imaging quality and robustness against model error. It explores the temporal modeling on both the image elements and electrode movements to reconstruct them within a single inverse. As the general forward model of EIT is $\mathbf{y}=\mathbf{J x}$, the upgraded forward model is illustrated as Figure 1.3; the temporal correlation model is described as same as Figure 1.1 .

4. Proposing a generalized iterative linearization method for EIT reconstructions which uses the $\ell_{1}$ norm minimization on both the regularization term and the residue error term.

$\ell_{1}$ norm calculates the sum of amplitude of a function. Generally, the $\ell_{1}$ norm of a function $f$ on a domain $\Omega$ is

$$
\|f\|_{1}=\int_{\Omega}|f|
$$

The $\ell_{1}$ norm regularization is advantageous over least squares methods by preserving discontinuities in the reconstructed profiles. This intrinsic property can be simply illustrated by a $1 \mathrm{D}$ special case in Figure 1.4. Suppose that there are multiple pathes between two 


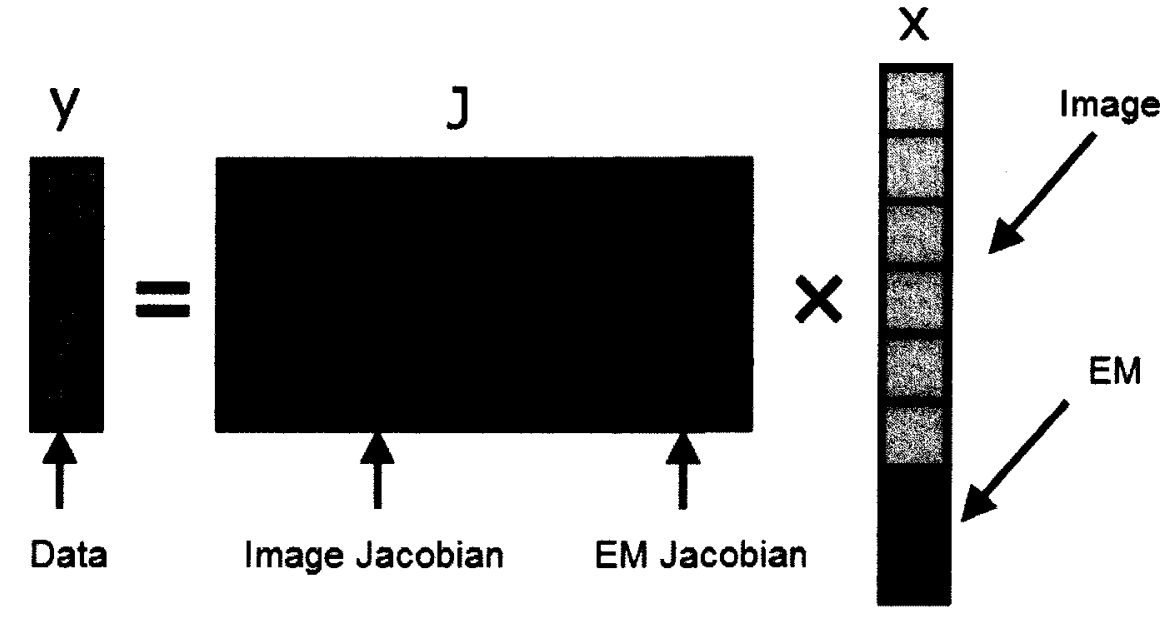

Figure 1.3: The augmented forward model for the reconstruction of both electrode movements and conductivity changes within a single inverse (Soleimani et al., 2006a). The electrode movement (EM) Jacobian and EM parameters are augmented to image Jacobian and image elements, respectively.

points $\mathrm{A}$ and $\mathrm{B}$, noted as functions $f_{1}(x), f_{2}(x)$ and $f_{3}(x)$, each of which is monotonically decreasing. By means of $\ell_{2}$ norm, the least squares solution is $f_{3}(x)$ due to the fact that the euclidean distance is the shortest distance between two points along any path. Thus for $\ell_{2}$ norm minimization on image prior term, any sharp edges of the type $f_{1}(x)$ will always be "smoothed" as $f_{3}(x)$ like transitions with largely decreased contrast. However, the $\ell_{1}$ norm of these pathes are identical:

$$
\|f\|_{1}=\int_{A}^{B}\left|f^{\prime}(x)\right| d x=f(A)-f(B)
$$

Therefore, being used as the image prior penalty of the Tikhonov regularization, $\ell_{1}$ norm minimization can preserves edges in original images.

Another promising advantage is that by applying $\ell_{1}$ norm minimization on the residue term, the solution has greater robustness against data outliers. Both advantages are achieved by applying $\ell_{1}$ norm minimization on both residue and regularization terms in a generalized $\ell_{1} / \ell_{2}$ iterative algorithm. 


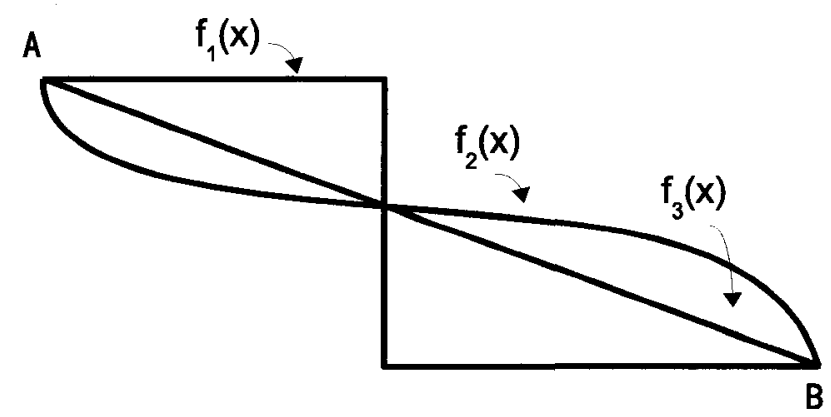

Figure 1.4: There are multiple paths between two points, $\mathrm{A}$ and $\mathrm{B}$. By means of $\ell_{2}$ norm minimization, the straight segment has the minimum value. However, there is no difference between difference pathes be means of $\ell_{1}$ norm solution.

\subsection{Contributions}

\subsubsection{Contributions by Objectives}

1. Determine the inter-image correlation and use it to improve EIT image performance

In this thesis, a novel temporal regularization for EIT is proposed. This approach reconstructs the image at frame $t_{0}$ from a frame window $t_{-d}$ to $t_{d}$ (as illustrated in Figure 1.1). The inversion is formulated as an inverse problem with a regularization prior which accounts for temporal correlations among images. For low noise solutions (low hyperparameter) its behaviour approximates that of Gauss-Newton reconstruction, while for cases of high noise level and high frame rates where large hyperparameters are adopted, it is advantageous by reconstructing higher resolution images. It improves over Kalman filter based algorithms by allowing an explicit control over the regularization prior and the data weighting methods. The temporal algorithm is recommended for cases where data is noisy and the signal (underlying conductivity changes) is slow with respect to the frame rate.

This work is presented in Chapter 3. Additionally, it is published as "Temporal Image Reconstruction in Electrical Impedance Tomography", Andy Adler, Tao Dai, William R.B. Lionheart, Physiol. Meas., 28:S1-S11, 2007 (Adler et al., 2007). My contribution was to partly derive the algorithm, write codes and conduct simulations.

As an extension, the temporal solver is also applied to the Magnetic Induction To- 
mography (MIT)(Soleimani et al., 2008). The inverse problem of MIT was treated as a dynamical problem that images non-static objects. The dynamic magnetic imaging of a molten metal flow is estimated with the aid of direct temporal imaging method. Through real data, it is proved that the temporal solver achieves better resolution than the conventional GN method especially for high noise cases.

2. Four dimensional (temporal and 3D spatial) correlations in the regularization prior of the GN one-step linearized reconstruction.

This algorithm considers the a priori spatial and temporal correlations in EIT imaging. The subject to be reconstructed is considered in 4D: as illustrated in Figure 1.2, elements of the 3D subject are correlated by an exponential model; the elements in the time axis are temporally correlated. For spatial correlations, a novel exponential model is presented based on: 1) sensitivity weighting, 2) an exponentially weighted model of inter-element correlations, and 3) a smoothness constraint for poor sensitivity regions. In order to avoid dependence on mesh element density, a closed form approximation to the integral is developed. For the temporal correlations, an exponential model is presented of inter-frame correlations (based on an exponential decay factor $\gamma$ ), and an automatic approach to determine $\gamma$ from the measurements is developed.

This work is presented in Chapter 4. Additionally, it is accepted as "EIT Image Reconstruction with 4-D Regularization", Tao Dai, Manuchehr Soleimani and Andy Adler, in Medical and Biological Engineering and Computing, 2008. (Dai et al., 2008b)

3. Temporal regularization on both the image elements and electrode movements to enhance imaging quality and robustness against model error.

This work is an extension and improvement to the temporal regularization and the electrode movement regularization approaches (proposed by Soleimani et al. 2006a), in this part, we develop an approach to reconstruct both the conductivity change and the electrode movements from the temporal sequence of EIT measurements. The electrode movement reconstruction is a powerful approach to reduce artefcats from boundary deformation. It calculates electrode displacements in addition to conductivity changes and is demonstrated to have high robustness against electrode mis-position. The reconstruction of both electrode movements and conductivity changes within a single inverse is illustrated 
in Figure 1.3. The electrode movement (EM) Jacobian and EM parameters are augmented to image Jacobian and image elements, respectively. The temporal inverse is formulated as an inverse problem with a regularization prior which accounts for both spatial and temporal correlations among image elements and electrode movements. The method is verified by a numerical simulation, saline phantom data and in vivo human measurement. Results show that this method improves quality of reconstructed images, both on noise performance and target resolutions.

This work is presented in Chapter 5. Additionally, it is published as "Reconstruction of Conductivity Changes and Electrode Movements Based on EIT Temporal Sequences", Tao Dai, Camille Gómez-Laberge and Andy Adler, Physiol. Meas., 29:S77-S88, 2008. (Dai et al., 2008a)

4. A generalized iterative linearization method for EIT reconstructions which use the $\ell_{1}$ norm regularization.

It is widely recognized that the $\ell_{1}$ norm regularization is good at recovering discontinuities in the true model while the least squares or $\ell_{2}$ solution is prone to smooth edges out. An efficient $\ell_{1}$ norm regularization method is of interest. However, the solutions of the $\ell_{1}$ norm regularization normally needed to solve an inverse problem as a minimization of a non-differentiable function which is computationally difficult. In this thesis, a generalized iterative reconstruction method for EIT is proposed. By choosing norm type values of $p_{n}$ and $p_{x}, \ell_{1}$ and $\ell_{2}$ norm minimization can be flexibly chosen on the data residue and/or image prior parts. Numerical simulations show the flexibility of this algorithm and merits of the $\ell_{1}$ norm solution on both imaging quality and robustness against sensor error.

This work is presented in Chapter 6. It is accepted as a conference presentation (Dai and Adler, 2008c), and is in progress to be submitted as a peer reviewed publication.

\subsubsection{Miscellaneous contributions}

Beside the primary research contributions described above, several correlated/preliminary researches are conducted: 
1. In bioimpedance imaging, spectroscopic modeling is a popular tool for bioelectrical characterization and parametric image establishment. In multifrequency EIT, modeled parameters can be used in stead of impedance values as elements. This thesis also conducted research on one implementation of bioimpedance spectroscopy. We develop a novel in vivo measurement technique to calculate bioelectrical properties of blood while excluding the contributions from surrounding tissues. This method is based on analysis of the difference spectroscopy measured from blood pooling. The spectroscopic data are fitted to a Cole model by Levenberg-Marquardt (LM) nonlinear curve fitting method. This approach is verified by an equivalent circuit simulation, a phantom experiment and a preliminary human test.

This work is presented in Chapter 7 and it is accepted to publish by IEEE Transactions on Instrumentation and Measurement, 2008. Authors are Tao Dai, Andy Adler.

2. As part of iterative system identification research, a temporally weighted and regularized optimal variable step size affine projection algorithm (APA) is proposed. This method proposes a forgetting weighted scheme on a projection matrix for variable step-size affine projection algorithms. It uses a forgetting processed input matrix as the projection matrix to estimate system deviation. This method introduces temporal weights into the projection matrix for the purpose of tracking the latest behaviour of error signal. This algorithm shows evident improvements, as tested by independent trials with coloured input signals and various parameter combinations.

This topic is discussed in Appendix A. This work is submitted to International Journal of Signal Processing, by Tao Dai, Andy Adler and Behnam Shahrrava, 2008. 


\section{Chapter 2}

\section{Background}

This chapter presents a review of knowledge pertinent to the thesis. Bioimpedance basics are discussed in terms of bioelectrical properties of biological tissues and primary applications of bioelectrical methods. The background of EIT is presented in terms of history, theory, and applications. Inverse problem basics and regularization techniques for EIT are discussed at the end of this chapter.

\subsection{Bioimpedance Basics}

Bioimpedance is a passive electrical property of a biological tissue which describes the opposition to current flow. For a stimulation current $I$ with a given frequency, the response voltage measured on the tissue is $V$. Both of them are complex numbers and can be expressed by polar forms, $I=|I| e^{j \angle I}, V=|V| e^{j \angle V}$. The electrical impedance, $Z=R+j X$, is a complex number which is calculated by the Ohm's law: $Z=V / I$, where $|Z|=|V| /|I|$ and $\angle Z=\angle V-\angle I$. The real part of $Z$ is the resistance $R$ and the imaginary part is the reactance $X . R$ causes the power attenuation and $X$ causes the phase difference between voltage and current.

In order to illustrate bioimpedance, consider a piece of cylindric material in which the impedance is measured through two ideally conductive plate electrodes at opposite sides, as illustrated in figure (2.1).

$$
Y=G+j B=G+j \omega C=K(\sigma+j \omega \varepsilon)=K\left(\sigma+j \omega \varepsilon_{r} \varepsilon_{0}\right)
$$

where symbols are explained below (units are in the parenthesis):

- $Y$ : the admittance which is the inverse of the impedance $Z(S)$ 


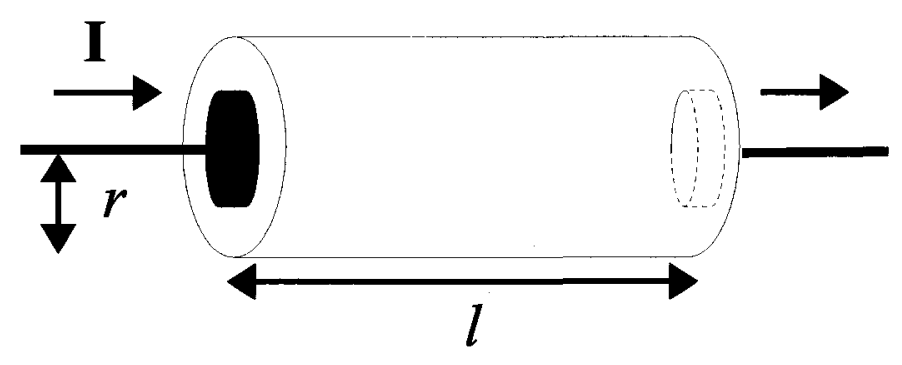

Figure 2.1: A piece of cylindric material to illustrate the relationship between bioelectrical properties and system geometry. This is formulated as the equation (2.1)

- $G$ : the conductance $(S)$

- $B$ : the susceptance $(S)$

- $C$ : the capacitance $(F)$

- $K$ : the scaling factor of the material measured(area/lengh $\left.=\pi r^{2} / l\right)(\mathrm{cm})$

- $\sigma$ : the conductivity of the material $(S / \mathrm{cm})$

- $\varepsilon$ : the permittivity of the material $(F / c m)$

- $\varepsilon_{r}$ : the relative permittivity of the material

- $\varepsilon_{0}$ : the permittivity of the vacuum as a constant $8.8 \times 10^{-14}(\mathrm{~F} / \mathrm{cm})$

Many electrical properties of tissue are related to polarization. Polarization is the electric field-induced disturbance of the charge distribution in a region. In a time varying electric field, the charge distribution is considered to change in time phase with the applied field. The time dependence can be characterized by the concept of "relaxation". Relaxation is the process of a system to relax to a new equilibrium after excited by a step signal. The time for the system to reach a new equilibrium is described as a parameter called "relaxation time".

The permittivity in biological materials typically diminishes with frequency increasing. The intrinsic reason is that the capability of charges following the variation of external electric field gets lower when frequency increases. As a correspondence of relaxation which 
describes biomaterial electrical properties with respect to time, the frequency dependence is described by "dispersion". Schawn (1957) introduced the concept of dispersion in the field of dielectric spectroscopic analysis of bio-materials. Dispersion can be understood as frequency dependence of permittivity according to certain biophysical mechanisms that are related to, such as cell membranes, intracellular organelles, bi-layer counterion relaxation, electrokinetic effects. Based on the observed permittivity dispersions, three dielectric dispersion regions $(\alpha, \beta$ and $\gamma)$ for the biological material were defined (as illustrated in figure 2.2)

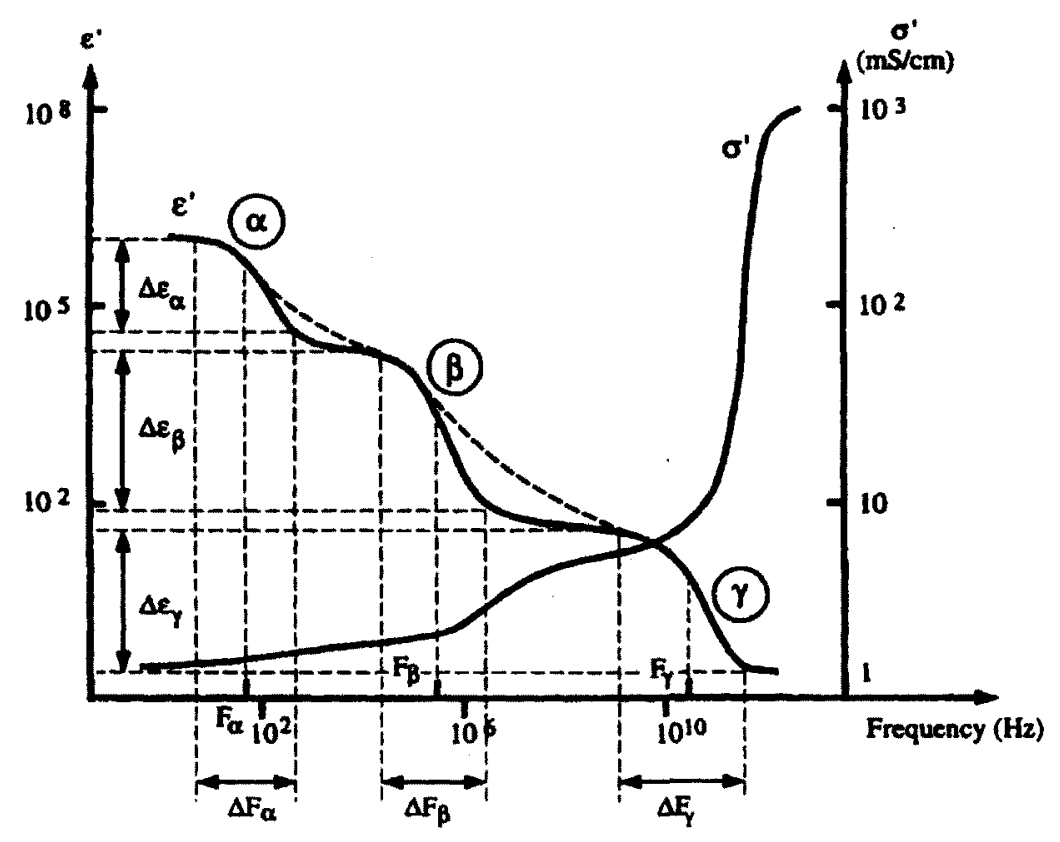

Figure 2.2: dielectric dispersion of biomaterials (from Valentinuzzi 1996). $\varepsilon^{\prime}$ is the relative permittivity (no unit) and $\sigma^{\prime}$ is the conductivity (unit: $m \sigma / \mathrm{cm}$ ).

Dielectric dispersions are generally considered to be related to these biophysical mechanisms (Foster and Schawn, 1989; Grimnes and Martinsen, 2000):

- The $\alpha$ dielectric dispersion (ranges from several $m H z$ to tens of $k H z$ ) is generally considered to be associated with counterion effects, active cell membrane effects, intracellular structures, the diffusion processes of the ionic species.

- The $\beta$ dispersion (ranges from $0.1 \mathrm{MHz}$ to $100 \mathrm{MHz}$ ) is related to the cell membranes, the extra-cellular and intra-cellular fluids. 
- The $\gamma$ dispersion (ranges from $0.1 \mathrm{GHz}$ to $100 \mathrm{GHz}$ ) region is attributed by the aqueous content of the biological species and small molecules.

The advantage of dispersion concept is that it is a general, phenomenological concept which facilitates researchers to focus on specific tissues in specific frequency band without considering detailed mechanisms (Gabriel et al., 1996a,b,c; Schepps and Foster, 1980).

\subsection{Bioimpedance Applications}

\subsubsection{Summary}

In medical area, bioimpedance method can be applied for many areas such as diagnostic and therapeutic applications, laboratory experiments and perception/hazard analysis. These application have advantages such as low-cost, easy to apply, portability and capable of on-line monitoring. Some existing applications are illustrated as below (Grimnes and Martinsen, 2000; Bourne et al., 1996):

- Cellular measurement

This method is to use impedance methods in the cellular field, for counting the amount of cells in a suspension, or estimating the concentration of dielectric particles in a conductive solution if the shape and size of the particles is known. For example, blood analyzers uses this approach to measure hematocrit.

- Body volume change and body composition measurement

As described in Section 2.1, the bioimpedance of a biomaterial is not only related with its properties but also depends on its geometrical dimensions. Therefore, as long as the material electrical properties are known in advance, it is possible to measure and calculate material sizes or volumes based on data. For example, impedance plethysmography uses bioimpedance data to estimate the blood volume in the extremities (Nyboer, 1970). In Chapter 7, a blood pooling based bioimpedance method for estimating blood bioelectrical properties is presented. There are some other similar examples like impedance cardiography and impedance pneumography.

The total body water can also be estimated by this method which calculates relative volumes of extra and intracellular spaces from bioimpedance measurement (Siconolfi et al., 1997; Thomas et al., 1992). The amount of body fat can be computed using the same techniques of the hydration monitoring. 
- Tissue identification and monitoring

Because different tissues exhibit different electrical properties, in addition, tissue electrical properties change with respect to tissue status evolution. Thus it is easy to conceive that bioimpedance method can be applied to identify and monitor tissues. This is discussed in details in Section 2.2.2 and Section 2.2.3.

- Electrical impedance tomography

Electrical Impedance Tomography (EIT) is the expansion of bioimpedance method to 2D/3D/4D applications by adding spatial and/or temporal resolution. EIT provides a mapping of the impedance distribution in a tissue layer or volume. This is the main topic of the thesis and detailed introduction is in Section 2.3.

\subsubsection{Bioimpedance method for tissue identification}

Since different tissues exhibit different bioelectrical characteristics, the bioimpedance method can be adopted to characterize tissues (as illustrated in figure 2.3 and table 2.1). For characterizing different tissues, one popular method is to fit data to the Cole-Cole model (Cole and Cole, 1941). The Cole equation models the behavior of permittivity and conductivity as a function of frequency:

$$
Z(f)=R_{\infty}+\frac{R_{0}-R_{\infty}}{1+j\left(f / f_{c}\right)^{1-\alpha}}
$$

where $R_{0}$ and $R_{\infty}$ are the limiting values of resistance at low and high frequencies; $f_{c}$ is the characteristic frequency of relaxation; and $\alpha$ is the constant that characterizes the Cole distribution function and ranges from 0 to 1 .

Table 2.1: typical Cole-Cole parameters of some tissues. From Rigaud et al. (1995).

\begin{tabular}{lllllll}
\hline & Muscle & Liver & Lung & Spleen & Intestine $(\alpha)$ & Intestine $(\beta)$ \\
\hline$f_{c}(\mathrm{kHz})$ & 27 & 72 & 140 & 373 & 520 & 2700 \\
$1-\alpha$ & 0.71 & 0.54 & 0.39 & 0.64 & 0.50 & 0.52 \\
$R_{0}(\Omega)$ & 640 & 605 & 390 & 525 & 135 & 110 \\
$R_{\infty}(\Omega)$ & 70 & 110 & 65 & 60 & 108 & 45 \\
\hline
\end{tabular}

One of the most attractive applications of bioimpedance characterization is cancer detection (Surowiec et al., 1988; da Silva et al., 2000; Halter et al., 2007). The cancerous 


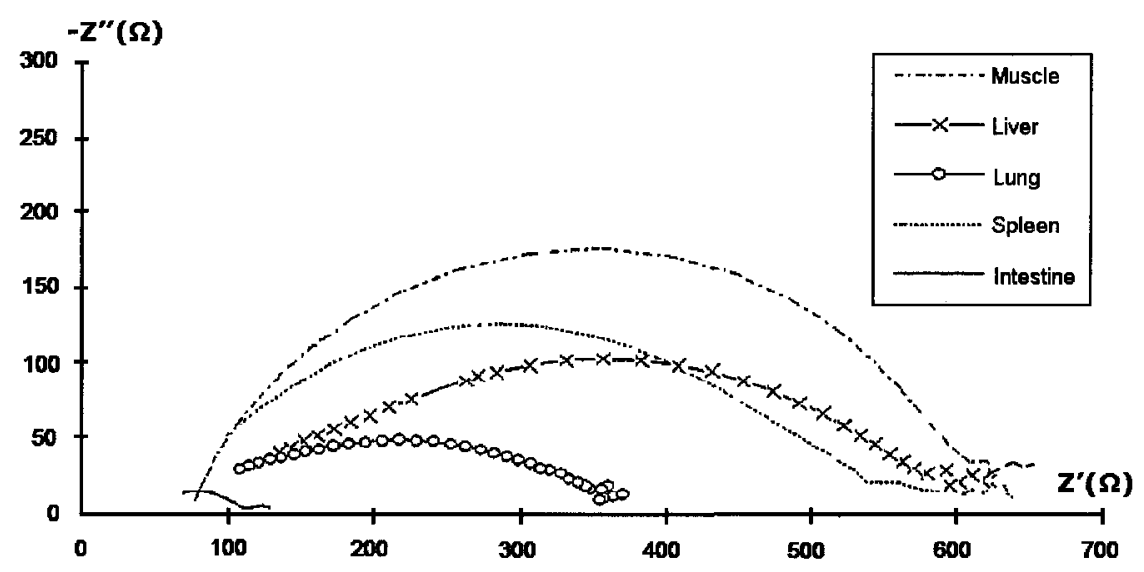

Figure 2.3: Typical cole-cole curves of some tissues. From Rigaud et al. (1995). $Z^{\prime}$ and $Z^{\prime \prime}$ are the real part and the imaginary part of the impedance, respectively.

tissues exhibit sharply different bioelectrical properties compared with the normal or benign tissues. When tumour tissue is in an early stage, abnormalities start to develop in cellular water/electrolyte content, cell membrane permeability, and orientation/packing density of cells; these changes introduce evident bioimpedance abnormalities that can be detected by using bioimpedance (Fricke and Morse, 1926; Scholz and Anderson, 2000). At this stage, absorbance properties of some high energy particle propagations, e.g., x-ray, of the cancerous tissue are relatively stable; therefore, this early stage abnormalities of cancerous tissues are difficult to be detected by this type of detections, e.g., CT.

The pioneer work of cancer detection using BIS is in the breast cancer diagnosis. As early as 1920', Fricke and Morse (1926) found significant capacitive difference between malignant breast tumors and normal tissues. Afterward, the potential usage of electrical characteristics to perform breast cancer detection was investigated both in vitro and in vivo: The relative permittivity and the conductivity of malignant tumours were found significantly different from the normal breast tissue (Surowiec et al., 1988; Jossinet, 1996; Jossinet and Schmitt, 1999); moreover, those researchers showed that benign tissues show similar bioelectrical properties to normal breast tissue. Morimoto et al. (1990) claimed that the malignant tissue has higher extracellular/intracellular resistance and lower membrane capacitance compared with benign tissue by using a three-electrode method. Kerner et al. (2002) showed that the BIS is promising for detecting breast malignancies by comparing BIS and mammogram results on human subjects. Kim et al. (2007) developed an analysis method to assess breast tissue and found the feasibility of adopting BIS 
on cancer detection by comparing Cole-Cole plots between malignant and healthy tissues. Based on bioimpedance, imaging modality had been proposed and the first commercialized bioimpedance imaging device was the TS2000 (Transcan Medical, Ltd., Israel; distributed by Siemens, Erlangen, Germany) which is used for breast cancer detection.

The bioimpedance cancer detection methodology is also an attractive topic of research on other tissues. For example, Walker et al. (2000) used a finite element model to simulate cell arrangement and electrical characteristics of premalignant cervical tissue, and found significantly different BIS curve compared with that from normal tissue. Halter et al. (2007, 2008a,b) conducted BIS measurement on different prostate compositions: prostate cancer, benign prostate hyperplasia, normal glandular tissue and stroma. Results showed that characteristic parameters mined from BIS data can differentiate cancerous and benign tissues which can only be fulfilled by sample biopsy currently. Smallwood et al. (2002) and Keshtkar et al. (2006) investigated the feasibility of applying the bioimpedance characterization to the bladder tissues to identify malignancies. They claimed that the bioimpedance technique can be a promising complementary method of current bladder abnormality diagnostic modalities.

\subsubsection{Bioimpedance method for tissue monitoring}

Tissue/organ monitoring is very important during surgery. For example, in some heart surgeries, the cardiac circulation is artificially bypassed and the heart becomes ischemic due to lack of oxygen. In order to prevent tissue damage, it is desired to determine how long this ischemia period can be. This decision can be made by timing, or else depending on experience of doctors. Some researchers have shown that the degree of heart ischemia can be evaluated by bioimpedance parameters (Casas et al., 1999; Linhart et al., 1995; Jenderka and Gersing, 1996), so that it provides an efficient and safe way to indicate the conditions of the heart.

Cryosurgery uses extreme cold for targeted destruction of undesirable tissues such as cancer. A cryoprobe is inserted into the malignant tissue and an ice ball grows on the top of probe to destroy malignant cells. In this procedure, accurate and real time monitoring of ice front extent is critical on destruction control. EIT is demonstrated a promising method for this application because frozen tissues show much higher impedivity than unfrozen tissues (Otten and Rubinsky, 2000, 2005; Edd et al., 2008).

Another example is to use bioimpedance parameters for monitoring organs to be transplanted to determine their suitability for transplantation. The idea is to quantify the 
damage caused by ischemia before, during and after the transplantation (Harms et al., 2001; Sola et al., 2003; Yamada et al., 2002).

\subsection{Electrical Impedance Tomography}

As discussed in Section 2.1, the difficulties of current propagating through various tissues within the object are different due to the bioelectrical heterogeneity within the measuring field. This principle makes it possible that a cross sectional (or three dimensional) impedance image can be reconstructed to provide informative anatomical and functional information of a medium.

Electrical Impedance Tomography (EIT) is an extension of the bioimpedance method described in the previous section by mapping bioelectrical properties (conductivity or permittivity) of a subject onto a two dimensional image or a three dimensional volume. It is a medical imaging medality in which an image of the internal conductivity/permittivity distribution of the body is reconstructed from boundary electrical measurements. Electrodes are attached to the surface of the subject and small alternating currents applied to some of the electrodes. The resulting electrical potentials are measured. This procedure repeats for different configurations of current stimulation to complete a data frame acquisition.

\subsubsection{History}

The earliest research on non-invasive imaging of human body in terms of bioelectrical properties was done by an impedance camera (Henderson and Webster, 1978). One voltage source was applied on human chest through a large area plate electrode to establish an equipotential surface on the chest; a twelve by twelve rectangular electrode array was attached on the thorax back and current data were measured behind each electrode on this array; the current data were used for generating thorax impedance images. The low conductive areas in the resulting images were claimed as lungs. Price (1979) tried to generate tomographic images by applying current stimulations and measuring voltages. The internal impedance distribution was solved by using a linear impedance network. The first two dimensional transverse plane impedance images were obtained by Barber and Brown (1984) using the first clinical impedance tomography system. Afterward, the Sheffield Mark 1 system was developed (Brown and Seagar, 1987) and this system was later commercialized. Currently, the Sheffield Mk 3.5 system is under developing and marketing by Maltron International Ltd., UK. Viasys Health Care (now Cardinal Health, Höchberg, 
Germany) manufactures Goe MF II EIT system for respiratory monitoring. Other EIT manufacturers include Dräger Medical (Lübeck, Germany) who has EIT system for respiratory monitoring; SIM-Technika (Moscow, Russian) who produces EIT devices for breast cancer screening.

\subsubsection{Principle of EIT}

In this section, general technical background knowledge EIT system is presented, such as signal excitation and data acquisition system, preliminary introduction of forward and inverse problem in EIT.

\section{Stimulation and Measurement}

Typically, during data acquisition of EIT, a set of electrodes are placed on the surface of the object following certain placement configuration. Current or voltage is applied to some electrodes and then resulting voltage or current is measured respectively. Mostly an alternating current is injected through some electrodes and the resulting voltages are measured at rest electrodes.

The current drive pattern is the way how the system is stimulated to generate responses. Sheffield protocol uses the adjacent pattern (Brown and Seagar, 1987) which applies alternating current through a pair of adjacent electrodes and the resulting voltages are measured at the remaining electrodes in adjacent pairs (as illustrated in figure 2.4 for respiratory monitoring application). The adjacent stimulation is easy to implement since only one current source is required and the current pair can be easily switched on other pairs by the control circuit. The optimal drive pattern was first introduced by Seagar (1983). Isaacson (1986) and Gisser et al. (1987) proposed to choose the optimal single current pattern by maximizing the $\ell^{2}$ norm of the difference between the measured and the calculated voltages, under the constraint of the $\ell^{2}$ norm of the current.

In single frequency EIT, a current pattern with certain frequency is injected into an imaging object and the boundary voltages are measured. Single frequency EIT applies a relatively low frequency, e.g., $50 \mathrm{kHz}$, at which the current travels mainly in the extracellular space and tissues being measured exhibit similar properties as direct current stimulation. However, at this frequency band, electrode impedance is much lower than that under direct current, such that the system sensitivity is better. Recently multifrequency EIT (MFEIT), or frequency difference EIT (fdEIT), has drawn more and more attentions of researchers. The MFEIT applies multifrequency stimulation thus an impedance spec- 


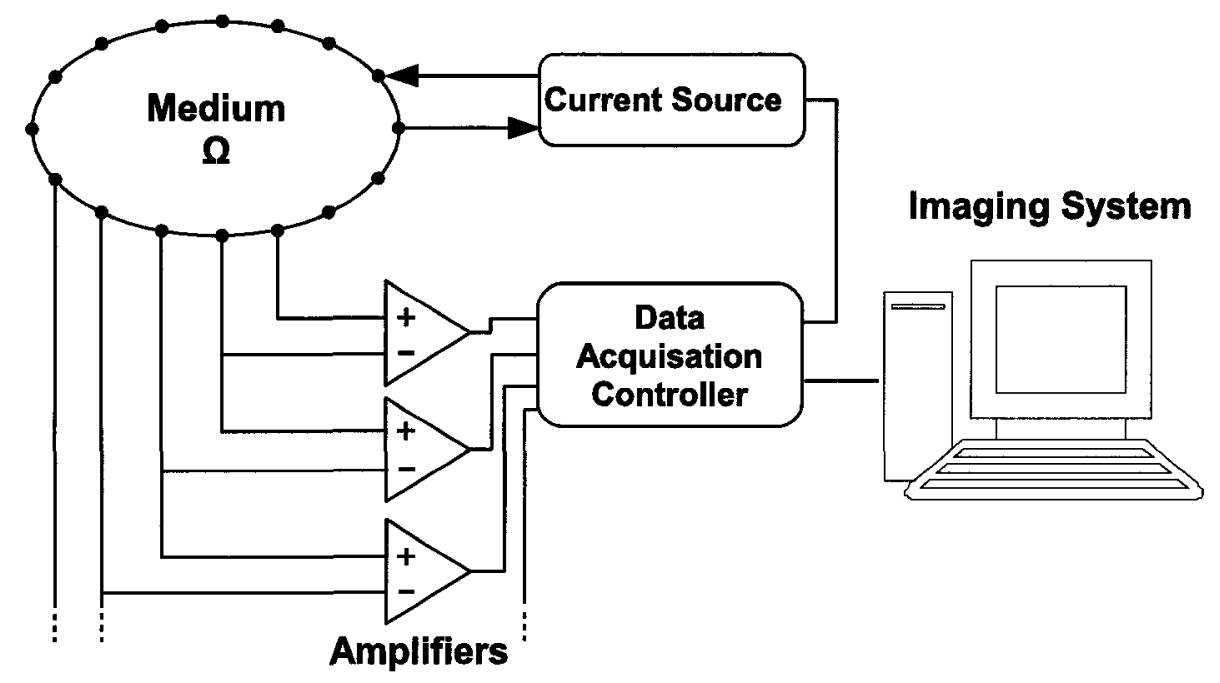

Figure 2.4: A block diagram of EIT system with 16 electrodes placed in a single plane, equidistantly. the adjacent stimulation and measurement pattern is applied: current is applied to an adjacent pair of electrodes and the responsive voltages are measured on the remaining 13 adjacent electrode pairs. Voltages are not measured on any stimulation electrodes. This procedure repeats 16 times until all 16 possible pairs of adjacent electrodes have been used to apply the known current. (Redrawn from Adler 1995)

trum is obtained for each pixel of reconstructed images. Instead of imaging conductivity changes with respect to time, MFEIT images conductivity changes with respect to frequency. Thus in principle the measurement is much less affected by subject movement and baseline drifting (Barber, 2005). In addition, images from high frequency are informative for cell membrane variation because current propagates not only extracellularly, but also intracellularly. This is promising for early stage cancer detection. The impedance difference between the cancerous tissue and normal tissue can be detected easier in high frequency band (low frequency EIT can only detect conductivity change of extracellular variation). Therefore, investigating MFEIT images within high band is meaningful for early stage cancer screening.

Using the MFEIT, it is possible to build an absolute image (either in conductivity or model parameters). For example, by using certain modeling methods, such as the ColeCole model, the bioimpedance parameters (such as the characteristic frequency $f_{c}$ ) can be estimated (Barber, 2005) and this can be used for tissue identification and monitoring (see Section 2.1). 


\section{Forwad Problem of EIT}

Given an object body $\Omega$ with a closed and smooth boundary $\partial \Omega$. Assuming the body has an isotropic conductivity distribution $\sigma(\mathbf{r})$ and the potential distribution is $\phi(\mathbf{r})$, both of them are functions of spatial locations $\mathbf{r}=(x, y, z)$. Assuming there is no internal current sources, $\sigma(\mathbf{r})$ and $\phi(\mathbf{r})$ are related by an elliptic partial differential equation, also known as the Laplace's equation

$$
\nabla \cdot \sigma \nabla \phi=0
$$

with the boundary condition $J_{0}=-\vec{J} \cdot \vec{n}=\sigma \nabla \phi \cdot \vec{n}$ where $\vec{J}=-\sigma \nabla \phi$ is the current density and $\vec{n}$ is the outward unit normal to $\partial \Omega$. Using (2.3) with the boundary condition that is specified by external sources, and the conductivity distribution inside the object, the potential distribution $\phi$ can be calculated on top of certain reference/ground point. This is the forward problem in EIT which is described as a nonlinear equation

$$
\mathbf{v}=F(\sigma, I)=F_{I}(\sigma)
$$

where $\sigma \in \mathbb{R}^{N}$ is the vector of internal conductivity of the medium being measured, if the conductivity image to be reconstructed has $N$ elements; $I$ is the current pattern injected into the medium; $\mathbf{v}$ is the voltage measured on the medium boundary; $F$ is the forward conversion.

Provided an EIT system with $n_{E}$ electrodes applied on a body surface in a plane. Supposing the Sheffield protocol is applied which uses the adjacent current stimulation and voltage measurement. $n_{E}$ current stimulation patterns are sequentially applied and each of them takes $n_{E}-3$ differential measurements (in this thesis, it is considered that voltages are not taken on stimulation electrodes). Each data frame measures a vector, $\mathbf{v} \in \mathbb{R}^{n_{M}}$, of $n_{M}=n_{E}\left(n_{E}-3\right.$ ) data points (half of which are redundant if the medium is not changing).

The difference EIT calculates difference data $\mathbf{y} \in \mathbb{R}^{n_{M}},\left(\mathbf{y}=\mathbf{v}_{t}-\mathbf{v}_{\mathbf{0}}\right)$, where $t$ is the time index. To improve its precision, the reference signal $\mathbf{v}_{0}$ is typically the average over many data frames that are acquired when the measured object may be assumed to be stable. Therefore, $\mathbf{v}_{0}$ is assumed to be noise-free. For cases that have no stable reference measuring condition (such as a patient who cannot hold breath to do the reference measurement), $\mathbf{v}_{0}$ can be estimated as the average of all data sets obtained during the periodic physiological activity (e.g., the lung ventilation monitoring in Chapter 5). The difference EIT calculates conductivity difference image $\mathbf{x} \in \mathbb{R}^{n_{N}}$ which is the difference of current conductivity $\sigma_{t}$ and the reference conductivity $\sigma_{0}$. A typical difference EIT image is illustrated as Figure 2.5 . 


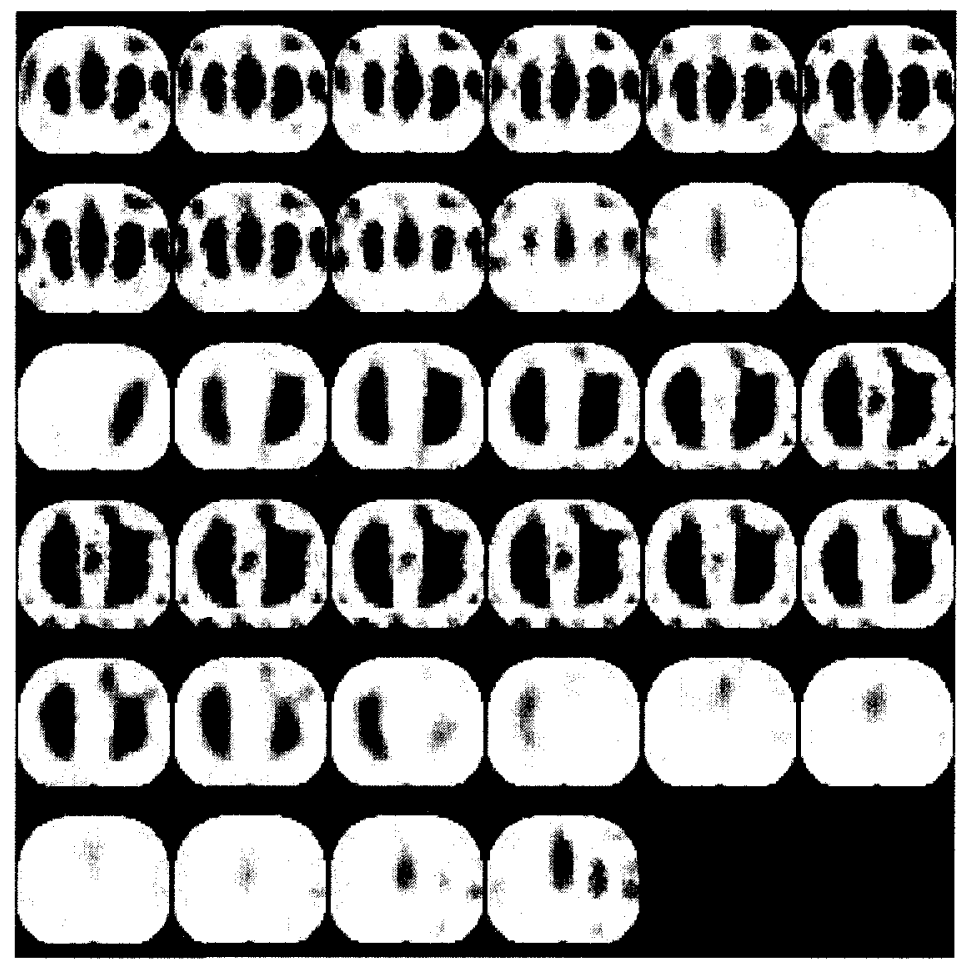

Figure 2.5: A typical EIT difference image (from online EIDORS tutorial, "Application: EIT of the thorax-Images of lungs", http://eidors3d.sourceforge.net/tutorial/tutorial.shtml). Lung ventilation image sequence (frame rate is $7 \mathrm{fps}$ ). Image progression is from left to right, top to bottom. One breath cycle is shown. The time average of the data set is used as the reference signal. Thus compared with reference lung volume (represented as blank image), blue regions represent less conductive (breath-in); red regions represent more conductive (breath-out).

For a small variation around $\sigma_{0}$, the relationship between $\mathbf{x}$ and $\mathbf{y}$ is approximately linear. Therefore, a linearized forward model can be formulated as

$$
\mathbf{y}=\mathbf{J} \mathbf{x}+\mathbf{n}
$$

where $\mathbf{n}$ is measurement noise that is assumed to be uncorrelated white Gaussian.

The linear operator $\mathbf{J}$ is the sensitivity matrix or the Jacobian matrix. On a model with $n_{E}$ elements and $n_{M}$ boundary measurements, $\mathbf{J} \in \mathbb{R}^{n_{M} \times n_{E}}$. The Jacobian matrix depends on the FEM, current injection patterns, the reference conductivity and the electrode models. It is calculated from a forward element model (FEM), which is built to simulate the medium being measured. This Jacobian is normally computed using perturbation technique, by introducing small amount of model property change and repeatedly solving the 
forward problem. For example, to calculate conductivity Jacobian near certain reference conductivity distribution $\mathbf{x}_{0}$, a small conductivity change $\triangle \mathbf{x}_{j}$ on the $j^{\text {th }}$ FEM element is made and this introduces voltage change on the $i^{\text {th }}$ measurement by an amount $\triangle \mathbf{y}_{i}$. The element on the $i, j$ position of the Jacobian is calculated as below (Adler and Guardo, 1996a)

$$
\mathbf{J}_{i j}=\left.\frac{\triangle \mathbf{y}_{i}}{\triangle \mathbf{x}_{j}}\right|_{\mathbf{x}_{0}}
$$

Other than conductivity Jacobian, electrode movement Jacobian can also be calculated using the perturbation technique by replacing the small conductivity perturbation in (2.6) by a small electrode position perturbation (Soleimani et al., 2006a). The perturbation technique is simple but computational expensive and inaccurate under certain circumstances. A direct method to calculate the Jacobian was proposed, based on a formulation of the derivatives of the FEM matrix with respect to geometry changes (Gómez-Laberge and Adler, 2008).

\section{Inverse Problem of EIT}

EIT image reconstruction attempts to inverse the problem of (2.5), in which $\mathbf{x}$ is estimated by solving

$$
\hat{\mathbf{x}}=\mathbf{J}^{-1} \mathbf{y}
$$

However, the solution (2.7) is not realizable because $\mathbf{J}$ is mostly not invertible. The linear inverse of (2.5) can be solved by minimizing the error function $\|\mathbf{J x}-\mathbf{y}\|$ in the least squares means as the generalized form

$$
\hat{\mathbf{x}}=\left(\mathbf{J}^{T} \mathbf{J}\right)^{-1} \mathbf{J}^{T} \mathbf{y}
$$

This is so called naïve least squares solution because $\mathbf{J}^{T} \mathbf{J}$ is rank-deficient and ill-conditioned (the term "ill-conditioned" refers to discrete linear system, while the term "ill-posed" refers to continuous system (Aster et al., 2005)). The rank-deficiency is caused by the fact that the number of unknown conductivity elements is mostly much larger than the number of independent measurements obtained. Thus this is an under-determined system which has infinite solutions and can not be directly solved by matrix inversion. There are also other factors which introduce difficulties for EIT reconstruction, such as poor sensitivity at the medium center, off-plane current propagation. The detailed inverse problem related to EIT is discussed in Section 2.4. 


\subsubsection{Applications}

EIT has been applied in many areas which can be classified as three major fields:

1. Biomedical imaging.

EIT has been applied for several medical applications: monitoring of pulmonary function (Adler, 1995; Frerichs, 2000; Harris et al., 1992; Kunst et al., 1998; Frerichs et al., 1999); measurement of cardiac function (Eyuboglu et al., 1989; Vonk-Noordegraaf et al., 2000), brain functions (Holder, 1992), gastric emptying (Smallwood et al., 1994); cancerous tissue detection and classification (Kerner, 2001; Halter et al., 2004; Zou and Guo, 2003).

2. Geophysical exploration.

EIT technique is used mainly on geological structure receptivity survey (Loke and Barker, 1996; Dines and Lytle, 1981). Electrodes are placed on the surface of the earth or in bore holes to position resistivity singularities.

3. Industrial process tomography.

Applications in industry include the imaging of conductive fluid flows in vessels and pipes (Beck et al., 1998; Ma et al., 2003; Hunt et al., 2003), crack detection (Williams and Beck, 1995; Dickin and Wang, 1996; Alessandrini and Rondi, 1998).

\subsection{Inverse Problem Theory}

An inverse problem is the task that often occurs in many scientific branches. Regarding a forward problem which is simply described as "model parameter" $\Rightarrow$ "data", an inverse problem can be described as "data" $\Rightarrow$ "model parameter". Inverse problems are typically ill-posed. This makes the solutions be sensitive to data and stabilization methods are required. This section introduces basic theory and solutions of the inverse problem related to EIT.

\subsubsection{Inverse Problem}

For one physical system with a parameter vector $\mathbf{x}$, observations $\mathbf{y}$ can be made through certain relationship $f$. The model is described as:

$$
\mathbf{y}=f(\mathbf{x})
$$


which is termed as forward problem. As long as we have the observations $\mathbf{y}$ and want to calculate the system parameters $\mathbf{x}$ through a inverse relations $f^{-1}$, this is so called inverse problem and described as:

$$
\mathbf{x}=f^{-1}(\mathbf{y})
$$

Unfortunately, for most of real world systems, the inverse solution may not be obtained as easy as the equation above because of ill-conditionness.

Regarding to the concept of a "well-conditioned" system, according to Jacques Hadamard (Hadamard, 1902; Tikhonov and Arsenin, 1977), a physical system is well-conditioned if all of the following conditions are satisfied:

- solution existence. For data obtained, there is a solution exists.

- solution uniqueness. The solution is unique.

- solution stability. The solution is continuous if data is continuous.

A system is realized ill-conditioned as long as it is not well-conditioned. There are several reasons for one real system to be ill-conditioned, e.g., the first condition may not be satisfied due to noise in data and/or modeling error; the second condition is not true for rank-deficient system because non-uniqueness of solutions is a characteristic of rankdeficient system; the process to calculate an inverse solution often extremely unstable which means even a little amount change in data will introduce extremely large amount of change in the model estimation-this fails the third condition.

To solve ill-conditioned problems numerically, a priori information about the solution, such as an assumption on the smoothness or a bound on the norm, is needed. This technique is the regularization. Regularization can avoid the unexpected situation of data over-fitting. Regularization applies a priori constraints to calculate "reasonable" solutions. The a priori constraints is so called "prior", such as statistical distribution of model parameters, global or local correlations of model parameters.

There are different regularization techniques, such as Tikhonov Regularization, truncated Singular Value Decomposition (SVD), Maximum Entropy Regularization (MER) and Total Variation (TV) regularization.

\section{Tikhonov Regularization}

The most widely applied regularization technique is the Tikhonov (or Tikhonov-Phillips) regularization. For the purpose of dealing with data "over-fitting" which introduce insta- 
bility of solutions, Tikhonov regularization uses additional information about the solution, commonly referred to as a prior. The prior is incorporated into the solution as an additional term in the least squares minimization. Instead of minimizing only the data fidelity term $\|\mathbf{J} \mathbf{x}-\mathbf{y}\|$, one regularization term is added in the form as below:

$$
\hat{\mathbf{x}}=\underset{x}{\arg \min }\left[\|\mathbf{J x}-\mathbf{y}\|^{2}+\lambda^{2}\left\|\mathbf{x}-\mathbf{x}_{0}\right\|^{2}\right]
$$

where $\lambda=\frac{\sigma_{n}}{\sigma_{x}}$ is a hyperparameter (or regularization parameter) that controls error weight between two terms. $\sigma_{\mathbf{x}}$ and $\sigma_{\mathbf{n}}$ are standard deviation of the unknowns $\mathbf{x}$ and standard deviation of the noise, respectively. $\mathbf{x}_{0}$ is the "reasonable" image known a priori. When $\lambda$ is close to zero, $\hat{\mathbf{x}}$ tends to the general least squares solution as (2.8). By taking derivative of the above equation and let it to zero, the solution is as:

$$
\hat{\mathbf{x}}=\left(\mathbf{J}^{T} \mathbf{J}+\lambda^{2} \mathbf{I}\right)^{-1} \mathbf{J}^{T} \mathbf{y}
$$

Which is the solution of Tikhonov regularization.

\section{Bayesian Interpretation of Tikhonov Regularization}

By defining the data $\mathbf{y}$ and image $\mathbf{x}$ as random variables, Bayes' theorem describes the following conditional probability relation between them

$$
P(\mathbf{x} \mid \mathbf{y})=\frac{P(\mathbf{y} \mid \mathbf{x}) P(\mathbf{x})}{P(\mathbf{y})}
$$

It is the posterior $\mathrm{P}(\mathbf{x} \mid \mathbf{y})$ (the possibility of $\mathbf{x}$, given $\mathbf{y}$ ) that is what we want to maximize for a "most likely" solution $\hat{\mathbf{x}}$ based on measured data $\mathbf{y}$.

Suppose $\mathbf{x}$ is independent with noise and assume that $\mathbf{x}$ is multivariate Gaussian with covariance matrix $\boldsymbol{\Sigma}_{x}$ and mean $\mathbf{x}_{0}$; the noise $\mathbf{n}$ has covariance matrix $\boldsymbol{\Sigma}_{n}$ and zero mean, then

$$
P(\mathbf{x} \mid \mathbf{y})=\frac{1}{P(\mathbf{y})} \exp \left(-\frac{1}{2}\|\mathbf{J} \mathbf{x}-\mathbf{y}\|_{\mathbf{\Sigma}_{n}^{-1}}^{2}\right) \exp \left(-\frac{1}{2}\left\|\mathbf{x}-\mathbf{x}_{0}\right\|_{\Sigma_{x}^{-1}}^{2}\right)
$$

This is so called maximum a posteriori (MAP) estimate. Where the subscript of the norm represents the weighting process as $\|\mathbf{a}\|_{\mathbf{B}}^{2}=\mathbf{a}^{T} \mathbf{B a}$. To maximize (2.14) is equivalently to minimize

$$
\|\mathbf{J} \mathbf{X}-\mathbf{y}\|_{\boldsymbol{\Sigma}_{n}^{-1}}^{2}+\left\|\mathbf{x}-\mathbf{x}_{0}\right\|_{\boldsymbol{\Sigma}_{x}^{-1}}^{2}
$$

Suppose $\mathbf{n}$ is uncorrelated white noise, $\boldsymbol{\Sigma}_{n}$ is a diagonal matrix with $\left[\boldsymbol{\Sigma}_{n}\right]_{i, i}=\sigma_{n}^{2}$, where $\sigma_{n}$ is standard deviation of noise. Similarly, if $\mathbf{x}$ is self-independent then $\boldsymbol{\Sigma}_{x}$ is a diagonal 
matrix with $\left[\boldsymbol{\Sigma}_{x}\right]_{i, i}=\sigma_{x}^{2}$, where $\sigma_{x}$ is standard deviation of image elements magnitude. Then the solution of (2.15) can be calculated as

$$
\hat{\mathbf{x}}=\left(\mathbf{J}^{T} \mathbf{J}+\left(\frac{\sigma_{n}}{\sigma_{x}}\right)^{2} \mathbf{I}\right)^{-1} \mathbf{J}^{T} \mathbf{y}
$$

Here (2.16) is the same as $(2.12)$.

\subsubsection{Inverse Problem on EIT}

EIT is a severly ill-conditioned system. The direct inverse solution can often be extremely unstable in that even a small amount of data noise can be largely amplified and ruin the entire estimation results.

Theoretically the solution existence is not suspectable because there must be a conductivity distribution which creates voltage measurement on boundary. The solution existence is also demonstrated in the general form as non-scattering objects and non-radiating sources, by Hoenders $(1978,1997)$. However, noise in data violates this consistency. In addition, there are some other errors may break this consistency such as electrode movement, the true model of EIT may not be correctly established. This violation can be partially

solved by searching for the best solution to minimize the distance between data and system forward solution. This provides an approximately accurate solution that is close to the true parameter set.

Due to the limitation of electrode size and placement restrictions, practically, the number of independent measurements made is always much less than the number of image elements to be estimated. Therefore EIT is rank-deficient. A rank-deficient system can have either no solution or infinite solutions. For EIT, because the solution existence has been guaranteed, it can then have infinite solutions. Thus the solution uniqueness does not hold for EIT. Other than the true solution, there are infinite "fake" solutions. One or some of these solutions can match the data even better than the true one. This case is so called "overfitting" in which a false model may fit perfectly to the data in that noises actually determined the main feature of system parameters.

The solution stability is not satisfied in EIT. Due to the limited measurement precision, even the most advanced measuring system may generate noise that causes arbitrarily large variation in the reconstructed conductivity distribution. For most cases in EIT, the solution instability is the hardest to deal with compared with the solution existence and the solution uniqueness (Lionheart et al., 2005). 
The approaches of calculating the proper inverse mapping and unique solution is generally termed as image reconstruction. The regularization is needed for image reconstruction of ill-conditioned system. This is the primary topic of this thesis.

\subsubsection{Regularization of EIT}

The regularization technique is widely used in EIT image reconstruction to deal with ill-conditionness of EIT. There are some recently developed nonlinear methods such as layer-stripping reconstruction (Somersalo et al., 1991; Cheney et al., 1992), monotonicity method (Tamburrino and Rubinacci, 2002), D-bar method (Siltanen et al., 2000; Isaacson et al., 2004, 2006). As the most popular image reconstruction methods in EIT, the regularization method is generally classified into three types: back-projection method, iterative method and one-step linearized method.

\section{back-projection}

Many early reconstructions were based on the back-projection method, such as the Sheffield Mark I (Brown and Seagar, 1987). The principle is similar to the back-projection reconstruction used in CT (Figure 2.6).

For EIT, each measured voltage is assumed to be proportional to the impedance between the driven and the measuring electrode pairs. Equipotential back-projection method (Barber and Brown, 1986) back-projects the impedance change recorded between two electrodes onto the reconstruction model (normally a 2D circular image), along the region defined by the equipotential lines ending on those electrodes. A full set of resulting back-projected regions are obtained from the procedure previously described and then overlapped to establish a reconstructed image (Figure 2.7). The back-projection method intrinsically introduces blur effect so that normally a filtering process is applied to compensate high frequency information afterward.

\section{Iterative method}

This method seeks a full reconstruction of the conductivity distribution using iterative approach which considers intrinsic nonlinearity. The linearization of EIT forward model (2.5) is valid only under the condition that the conductivity changes are of low amplitudes. This condition holds for small changes below $\pm 20 \%$ of the reference value (Holder, 2005). However, for some physiological activities such as pulmonary ventilation during deep breathing, this condition is not valid any more. The nonlinear method is applicable 


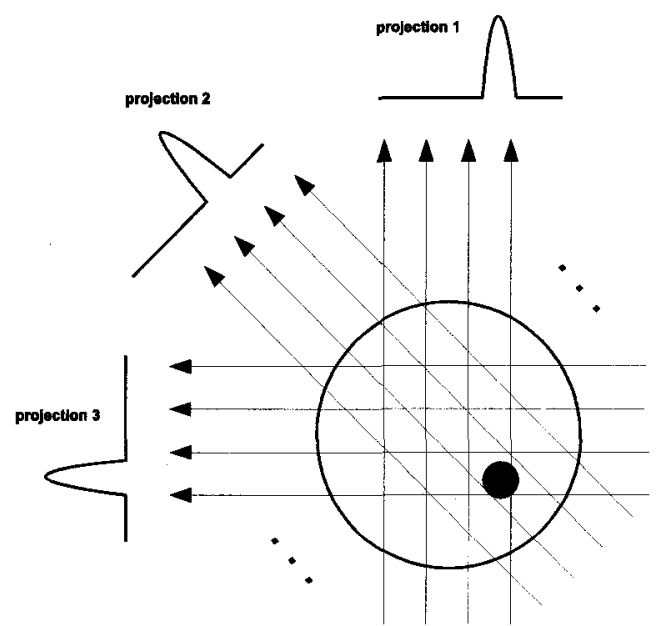

(a)

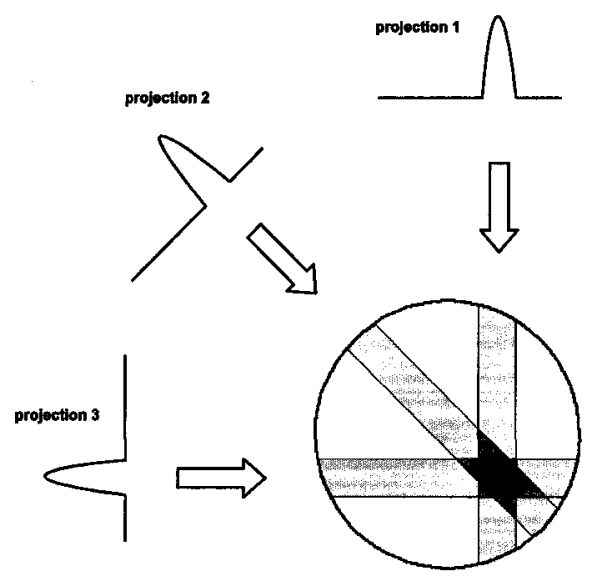

(b)

Figure 2.6: Principle of CT image reconstruction by back-projection. (a) Forward projection of CT. The target has higher x-ray absorbance coefficient than surroundings. Projection profiles are signal strength collected at the projection ends (profiles are inverted); (b) back-projection of CT. The target is reconstructed by back-projecting profiles onto an image plane, with lower contrast though.

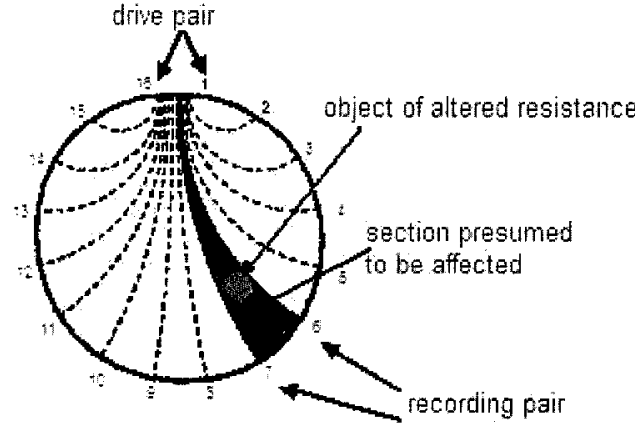

(a)

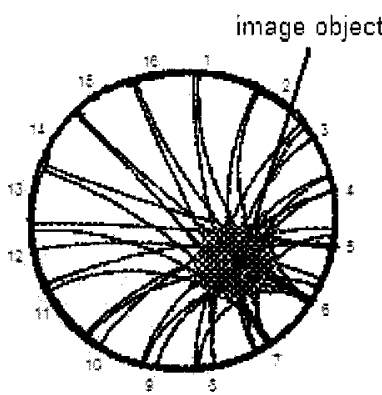

(b)

Figure 2.7: Principle of EIT image reconstruction by back-projection that is based on equipotential region (from Holder 2005). (a) Forward projection of EIT. (b) back-projection of EIT. The target is reconstructed by back-projecting multiple measurements onto an image plane along equipotential regions. 
for these cases (with certain conditions apply).

It works as follows (as illustrated in Figure 2.8): first an estimation of the conductivity distribution is made; then calculate the estimated voltage values through forward solution; compare the estimated values with the original recorded voltage data and the error is used to calculate the deviation between the real and the estimated conductivities; the conductivity vector estimated is then be adjusted. This procedure is repeated until the error between the estimated and recorded voltages is minimized to an acceptable level. This method provides more accurate but slower solution compared with the one step linear reconstruction, so that it is suitable for anatomical imaging. However, iterative method is argued to be sensitive to errors, e.g., mismatch between true anatomy of subject and the reconstruction mesh, electrode movement and contact impedance drift (Lionheart, 2004; Morucci and Marsili, 1996). These errors accumulate through iterations and this may introduce instabilities. Therefore, a recommendation is that iterative methods are good for cases where subjects have well-known anatomic structures and in relatively stable status, such as tank measurement, and one-step reconstruction is better for in vivo, dynamic system measurements.

\section{One-step linear reconstruction}

This approach simplifies the solution as

$$
\hat{\mathbf{x}}=\mathbf{B y}
$$

It addresses the inverse solution as a linear reconstruction matrix $\mathbf{B}$ and allows use of advanced regularization methods to solve the inverse problem.

The Gauss-Newton (GN) method in EIT (Yorkey et al., 1987; Cheney et al., 1990; Adler and Guardo, 1996a) estimates a solution $\hat{\mathbf{x}}$ by minimizing

$$
\|\mathbf{y}-\mathbf{J} \mathbf{x}\|_{\Sigma_{n}^{-1}}^{2}+\left\|\mathbf{x}-\mathbf{x}_{0}\right\|_{\Sigma_{x}^{-1}}^{2}
$$

where $\boldsymbol{\Sigma}_{n} \in \mathbb{R}^{n_{M} \times n_{M}}$ is the covariance matrix of the measurement noise $\mathbf{n}$. Since noise channels are independent, $\boldsymbol{\Sigma}_{n}$ is a diagonal matrix with $\left[\boldsymbol{\Sigma}_{n}\right]_{i, i}=\sigma_{i}^{2}$, where $\sigma_{i}^{2}$ is the noise variance at channel $i$. Here $\boldsymbol{\Sigma}_{x} \in \mathbb{R}^{n_{N} \times n_{N}}$ is the covariance matrix of the desired image and $\mathbf{x}_{0}$ represents the expected value of image (for difference EIT, $\mathbf{x}_{0}=\mathbf{0}$ ).

$\boldsymbol{\Sigma}_{n}$ and $\boldsymbol{\Sigma}_{x}$ are heuristically modelled a priori. Let $\mathbf{W}=\sigma_{n}^{2} \boldsymbol{\Sigma}_{n}^{-1}$ and $\mathbf{R}=\sigma_{x}^{2} \boldsymbol{\Sigma}_{x}^{-1}$. Here $\sigma_{n}$ is the mean of noise amplitude and $\sigma_{x}$ is the a priori amplitude of image element values. 


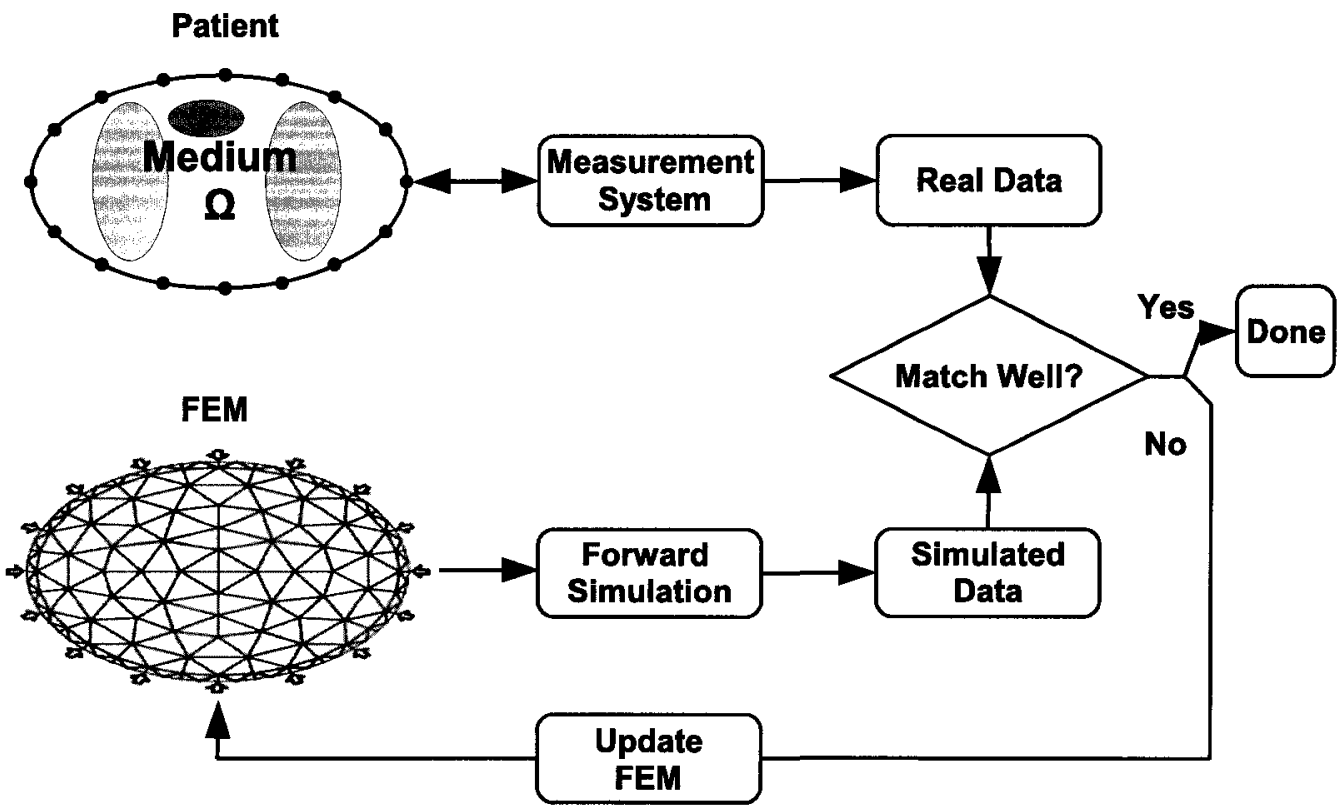

Figure 2.8: Iterative reconstruction of EIT. (Redrawn from Adler (1995)). Real measured data are compared with simulated data from FEM. The error signal is quantified and used to calculate a conductivity adjustment that is used to update conductivity distribution of FEM. This procedure repeats until the error signal is small enough.

The measurement accuracy is modelled by $\mathbf{W}$. For uncorrelated noise, each diagonal element of $\mathbf{W}$ is proportional to the corresponding channel signal-to-noise-ratio (SNR). For difference EIT with identical channels, $\mathbf{W}$ is an identity matrix; or else, it may be obtained during the system calibration test, e.g., by measuring on a subject with stable conductivity and recording noise variances of all channels. The matrix $\mathbf{R}$ statistically models the amplitudes and interactions of image elements. Simply, $\mathbf{R}$ may consider all elements have identical mean amplitude and independent with each other (or only locally dependent). More sophisticated models (Dai et al., 2008a) (Chapter 4) also consider global spatial dependency and temporal correlation between adjacent images.

From (2.18), a linearized one-step inverse solution is obtained as

$$
\hat{\mathbf{x}}=\left(\mathbf{J}^{T} \mathbf{W} \mathbf{J}+\lambda^{2} \mathbf{R}\right)^{-1} \mathbf{J}^{T} \mathbf{W} \mathbf{y}=\mathbf{B} \mathbf{y}
$$

where $\lambda=\sigma_{n} / \sigma_{x}$ is the regularization parameter, or hyperparameter, which controls the trade-off between resolution and noise attenuation in the reconstructed image. Here $\mathbf{B}=$ $\left(\mathbf{J}^{T} \mathbf{W} \mathbf{J}+\lambda^{2} \mathbf{R}\right)^{-1} \mathbf{J}^{T} \mathbf{W}$. 
Assuming that image elements are independent and have an identical expected magnitude, $\mathbf{R}$ becomes an identity matrix $\mathbf{I}$ and (2.19) uses zeroth order Tikhonov regularization (2.12). For EIT, such solutions tend to push reconstructed noise toward boundary, because data are much more sensitive to boundary elements than internal elements. One compensation scheme of this sensitivity discrepancy is to scale $\mathbf{R}$ by the sensitivity of elements, such as the method of NOSER prior (Cheney et al., 1990).

Because the reconstruction matrix $\mathbf{B}$ can be precalculated off-line, this one-step linearized reconstruction results in a fast solution which is applicable for real time functional imaging. However, the disadvantage is that it is less accurate than iterative method. 


\section{Chapter 3}

\section{Temporal Image Reconstruction in Electrical Impedance Tomography}

\subsection{Summary}

This chapter is based on the paper - Temporal Image Reconstruction in Electrical Impedance Tomography, published in Physiol. Meas. by Andy Adler, Tao Dai and William R.B. Lionheart (Adler et al., 2007).

Electrical Impedance Tomography (EIT) calculates images of the body from body impedance measurements. While the spatial resolution of these images is relatively low, the temporal resolution of EIT data can be high. Most EIT reconstruction algorithms solve each data frame independently, although Kalman filter algorithms track the image changes across frames. This chapter proposes a new approach which directly accounts for correlations between images in successive data frames. Image reconstruction is posed in terms of an augmented image $\tilde{\mathbf{x}}$ and measurement vector $\tilde{\mathbf{y}}$, which concatenate the values from the $d$ previous and future frames. Image reconstruction is then based on an augmented regularization matrix $\tilde{\mathbf{R}}$, which accounts for a model of both the spatial and temporal correlations between image elements. Results are compared for reconstruction algorithms based on independent frames, Kalman filters, and the proposed approach. For low values of the regularization hyperparameter, the proposed approach performs similarly to independent frames, but for higher hyperparameter values, it uses adjacent frame data to reduce reconstructed image noise. 


\subsection{Introduction}

Electrical Impedance Tomography (EIT) calculates an estimate of the conductivity distribution within a body based on current stimulations and voltage measurements on the body surface. EIT has fairly low spatial resolution, limited by the low sensitivity of surface measurements to conductivity changes deep within the body. On the other hand, EIT has excellent temporal resolution, with some recent systems having frame rates up to $1000 / \mathrm{s}$ (Wilkinson et al., 2005). Such high temporal resolution makes EIT a promising technology to monitor fast physiological events which affect the conductivity distribution. For cardiac activity, the frequency content of the QRS complex is mainly between $10-25 \mathrm{~Hz}$ (Kohler et al., 2002). Another example is high frequency ventilation in which air is pumped into the lungs at rates of $5-25 \mathrm{~Hz}$ (with smaller tidal volumes). High frequency ventilation is indicated for many patients with respiratory distress syndrome since it is understood to place less stress on injured lung tissue (Eichenwald and Stark, 1999). EIT can potentially be of great benefit to these patients, since the distribution of ventilation in their lungs is highly non-uniform and cannot be otherwise monitored (Wolf and Arnold, 2005).

Like many biomedical instrumentation techniques, the ability of EIT to see small physiological changes is limited by the signal to noise ratio (SNR). A widely used technique to improve SNR is ensemble averaging, which reduces random noise by the square root of the number of averaged frames. If EIT data acquisition is sufficiently rapid compared to the underlying physiological processes to be imaged, then ensemble averaging may be used on multiple frames of EIT. However, in EIT applications where conductivity changes are very fast with respect to the EIT frame rate, ensemble averaging is not appropriate, since it will effectively reduce the temporal resolution. In these cases, each frame of EIT data is typically reconstructed independently of the others.

In this chapter, we are interested in approaches to image a body which is undergoing fast changes with respect to the EIT frame rate. In these cases, ensemble averaging is not appropriate; however, it is clear that individual data frames are not completely independent, but do contain useful correlations, which could be exploited to improve EIT image noise performance. We call an approach which uses the time sequence of EIT frame data temporal image reconstruction.

Temporal image reconstruction can be represented as a linear tracking problem, and formulated as an extended Kalman filter, in which the image at each instant is estimated from the current data and the previous image estimate. Vauhkonen et al. (1998a) proposed the first Kalman filter based algorithm for difference EIT; we describe this approach using 
the notation of this chapter in section 3.3.3. More recently, this approach has been extended by Kim et al. (2004) to reconstruct the resistivity of a contrast of known shape and location. Kim et al. (2006) also proposed a computationally efficient algorithm based on pre-computing the Kalman gain and state estimation matrices. An algorithm for absolute EIT has also been shown for simulation data (Trigo et al., 2004).

In this chapter, we propose a new approach for temporal EIT image reconstruction, which directly estimates the image at frame $t_{0}$ from the set of data in a window of frames from $t_{-d}$ to $t_{d}$. Using these data, the temporal inverse is formulated as an inverse problem with a regularization prior which accounts for both spatial and temporal correlations between image elements.

\subsection{Methods}

We consider an EIT system with $n_{E}$ electrodes applied to a body using sequential current stimulation with parallel voltage measurement. Using these electrodes, $n_{E}$ current stimulation patterns are sequentially applied and $n_{V}$ differential measurements are made for each stimulation. For an adjacent drive EIT system, voltages are typically not measured at driven electrodes, and $n_{V}=n_{E}-3$. The delay between each successive stimulation pattern is $t_{S}$; thus, a complete set (frame) of EIT measurements takes time $t_{F}=n_{E} t_{S}$. Each data frame measures a vector, $\mathbf{v} \in \mathbb{R}^{n_{M}}$, of $n_{M}=n_{E} n_{V}$ data points (some of which are redundant if the medium is not changing). Difference EIT calculates difference data $\mathbf{y},\left([\mathbf{y}]_{i}=\left[\mathbf{v}_{2}\right]_{i}-\left[\mathbf{v}_{1}\right]_{i}\right) ;$ or the normalized difference data $[\mathbf{y}]_{i}=\left(\left[\mathbf{v}_{2}\right]_{i}-\left[\mathbf{v}_{1}\right]_{i}\right) /\left[\mathbf{v}_{1}\right]_{i}$. To improve its precision, $\mathbf{v}_{1}$ is typically averaged over many data frames, at a time when the conductivity distribution may be assumed to be stable; we thus assume that $\mathbf{v}_{1}$ is noise free.

The body under investigation is modelled using a finite element model (FEM) which discretizes the conductivity onto $n_{N}$ piecewise smooth elements, represented by a vector $\sigma \in \mathbb{R}^{n_{N}}$ (In this paragraph, $\sigma$ represents conductivity; elsewhere in this chapter, $\sigma$ is the standard deviation). Again, difference EIT calculates a vector of conductivity change, $\mathbf{x}=\sigma_{2}-\sigma_{1}$ between the present conductivity distribution, $\sigma_{2}$, and that at the reference measurement, $\sigma_{1}$. For small variations around the reference conductivity $\sigma_{1}$, the relationship between $\mathbf{x}$ and $\mathbf{y}$ can be linearized (giving the difference EIT forward model):

$$
\mathbf{y}=\mathbf{J x}+\mathbf{n}
$$

where $\mathbf{J} \in \mathbb{R}^{n_{M} \times n_{N}}$ is the Jacobian or sensitivity matrix and $\mathbf{n} \in \mathbb{R}^{n_{M}}$ is the measure- 
ment noise which is assumed to be uncorrelated white Gaussian. J is calculated from the

FEM as $\mathbf{J}_{i j}=\left.\frac{\partial \mathbf{y}_{i}}{\partial \mathbf{x}_{j}}\right|_{\sigma_{1}}$, and depends on the FEM, current injection patterns, the reference conductivity, and the electrode models. This system is underdetermined since $n_{N}>n_{M}$. This problem is commonly solved using regularization techniques (Cheney et al., 1990; Adler and Lionheart, 2006) in order to calculate a conductivity change estimate, $\hat{\mathbf{x}}$, which is both faithful to the measurements, $\mathbf{y}$, and to a priori constraints on a "reasonable" image.

Over time steps, $k$, a sequence of difference vectors, $\mathbf{y}_{k}=\mathbf{J x}_{k}$, are measured (assuming the body and electrode geometry, and thus $\mathbf{J}$, stay fixed). If the conductivity of the body under investigation doesn't change too rapidly, then it is reasonable to expect that a certain number of measurements, $d$, into the past and future provide useful information about the current image. Labelling the current instant as $t$, we therefore seek to estimate $\hat{\mathbf{x}}_{t}$ from data $\left[\mathbf{y}_{t-d} ; \ldots ; \mathbf{y}_{t-1} ; \mathbf{y}_{t} ; \mathbf{y}_{t+1} ; \ldots ; \mathbf{y}_{t+d}\right]$.

In the subsequent sections we consider three traditional approaches and the proposed temporal inverse; each estimates $\hat{\mathbf{x}}_{t}$ at frame $t$ from a sequence of data starting at frame 0 , using the indicated data: 5.3.1) Gauss-Newton (GN) inverse, using $\mathbf{y}_{t}$ only; 3.3.2) GN with weighted data, using a weighted average of $\left.\mathbf{y}_{t-d} \ldots \mathbf{y}_{t+d} ; 3.3 .3\right)$ Kalman filter inverse, using all previous and current data, $\mathbf{y}_{0} \ldots \mathbf{y}_{t}$; and 3.3.4) Temporal inverse, using $\mathbf{y}_{t-d} \ldots \mathbf{y}_{t+d}$ based on a temporal prior model.

\subsubsection{One-step linear GN(Gauss-Newton) solver}

One-step Gauss-Newton (GN) EIT reconstruction approaches have been widely used in EIT, e.g., Cheney et al. (1990); Adler and Guardo (1996a). They allow use of sophisticated regularized models of the EIT inverse problem, are able to represent this solution as a linear reconstruction matrix, which can then allow rapid, real-time imaging. The GN inverse problem seeks to calculate a solution, $\hat{\mathbf{x}}$, to the EIT inverse problem expressed as the minimum of a sum of quadratic norms

$$
\|\mathbf{y}-\mathbf{J} \mathbf{x}\|_{\Sigma_{n}^{-1}}^{2}+\left\|\mathbf{x}-\mathbf{x}^{\circ}\right\|_{\Sigma_{x}^{-1}}^{2}
$$

where $\mathbf{x}^{\circ}$ represents the expected value of element conductivity changes, which is zero for difference EIT. $\boldsymbol{\Sigma}_{n} \in \mathbb{R}^{n_{M} \times n_{M}}$ is the covariance matrix of the measurement noise $\mathbf{n}$. Since $\mathbf{n}$ is uncorrelated, $\boldsymbol{\Sigma}_{n}$ is a diagonal matrix with $\left[\boldsymbol{\Sigma}_{n}\right]_{i, i}=\sigma_{i}^{2}$, where $\sigma_{i}^{2}$ is the noise variance at measurement $i . \Sigma_{x} \in \mathbb{R}^{n_{N} \times n_{N}}$ is the expected image covariance.

Typically, $\boldsymbol{\Sigma}_{n}$ and $\boldsymbol{\Sigma}_{x}$ are not calculated directly. Instead, their inverses, $\mathbf{W}=\sigma_{n}^{2} \boldsymbol{\Sigma}_{n}^{-1}$ and $\mathbf{R}=\sigma_{x}^{2} \boldsymbol{\Sigma}_{x}^{-1}$, are heuristically determined from a priori considerations. Here $\sigma_{n}$ is 
the average measurement noise amplitude and $\sigma_{x}$ is the a priori amplitude of conductivity change. W models the measurement accuracy. For uncorrelated noise, each diagonal element is proportional to the signal to noise ratio. For difference EIT with identical channels, $\mathbf{W}=\mathbf{I}$. The regularization matrix $\mathbf{R}$ may be understood to model the "unlikelihood" of image elements.

By solving (3.2), a linearized, one-step inverse solution is obtained as

$$
\hat{\mathbf{x}}=\left(\mathbf{J}^{T} \frac{1}{\sigma_{n}^{2}} \mathbf{W J}+\frac{1}{\sigma_{x}^{2}} \mathbf{R}\right)^{-1} \mathbf{J}^{T} \frac{1}{\sigma_{n}^{2}} \mathbf{W} \mathbf{y}
$$

We define the hyperparameter $\lambda=\sigma_{n} / \sigma_{x}$, and rewrite (3.3) as

$$
\hat{\mathbf{x}}=\left(\mathbf{J}^{T} \mathbf{W} \mathbf{J}+\lambda^{2} \mathbf{R}\right)^{-1} \mathbf{J}^{T} \mathbf{W} \mathbf{y}=\mathbf{B y}
$$

where $\mathbf{B}=\left(\mathbf{J}^{T} \mathbf{W} \mathbf{J}+\lambda^{2} \mathbf{R}\right)^{-1} \mathbf{J}^{T} \mathbf{W}$ is the linear, one-step inverse. The regularization hyperparameter $\lambda$ controls the trade-off between resolution and noise attenuation in the reconstructed image.

If image elements are assumed to be independent with identical expected magnitude, $\mathbf{R}$ becomes an identity matrix I and (3.4) uses zeroth-order Tikhonov regularization. For EIT, such solutions tend to push reconstructed noise toward the boundary, since the measured data are much more sensitive to boundary image elements. Instead, $\mathbf{R}$ may be scaled with the sensitivity of each element, so that each diagonal element $i$ of $\mathbf{R}$ is $[\mathbf{R}]_{i, i}=\left[\mathbf{J}^{T} \mathbf{J}\right]_{i, i}^{p}$. This is the NOSER prior of Cheney et al. (1990) for an exponent $p=1$. Many other prior matrices have been proposed: to model image smoothness as a penalty for non-smooth image regions, $\mathbf{R}$ may be set to the discrete Laplacian filter (Vauhkonen et al., 1998b) or a discrete high pass Gaussian filter (Adler and Guardo, 1996a).

In this chapter, the NOSER prior is used for calculating the matrix $\mathbf{R}$ with $p=0.5$ in all tested algorithms, except for the Kalman filtering. Because it is diagonal, $\mathbf{R}$ can be inverted without numerical difficulties. The choice of exponent is a heuristic compromise between the pushing noise to the boundary $(p=0)$ or to the centre $(p=1)$.

In (3.4), the term in the inverse is of size $n_{N} \times n_{N}$. To save computational time, and improve inverse accuracy and stability, we want to decrease the size of the matrix to be inverted. Thus, we rewrite the matrix $\mathbf{B}$ using the data form as:

$$
\begin{array}{r}
=\left(\mathbf{J}^{T} \mathbf{W J}+\lambda^{2} \mathbf{R}\right)^{-1} \mathbf{J}^{T} \mathbf{W}\left[\left(\mathbf{J} \frac{1}{\lambda^{2}} \mathbf{P} \mathbf{J}^{T}+\mathbf{V}\right)\left(\mathbf{J} \frac{1}{\lambda^{2}} \mathbf{P J} \mathbf{J}^{T}+\mathbf{V}\right)^{-1}\right] \\
=\left(\mathbf{J}^{T} \mathbf{W} \mathbf{J}+\lambda^{2} \mathbf{R}\right)^{-1}\left(\mathbf{J}^{T} \mathbf{W} \mathbf{J}+\lambda^{2} \mathbf{R}\right)\left(\frac{1}{\lambda^{2}} \mathbf{P J}\right)\left(\mathbf{J} \frac{1}{\lambda^{2}} \mathbf{P J}^{T}+\mathbf{V}\right)^{-1} \\
=\mathbf{P J}^{T}\left(\mathbf{J P J} \mathbf{J}^{T}+\lambda^{2} \mathbf{V}\right)^{-\mathbf{1}}
\end{array}
$$


where $\mathbf{P}=\mathbf{R}^{-1}=\frac{1}{\sigma_{x}^{2}} \boldsymbol{\Sigma}_{x}$ and $\mathbf{V}=\mathbf{W}^{-1}=\frac{1}{\sigma_{n}^{2}} \boldsymbol{\Sigma}_{n}$. In practice, $\mathbf{P}$ and $\mathbf{V}$ are modelled directly from the system covariances, rather than the inverse of $\mathbf{R}$ and $\mathbf{W}$. Using (3.5), the size of the term in the inverse is reduced to $n_{M} \times n_{M}$. This is especially significant for 3D EIT models and for the temporal inverse which we introduce below.

Note that the GN solver does not consider the time sequence of EIT data. Each frame is solved individually, and inter-frame correlations are ignored.

\subsubsection{One-step linear GN solver with weighted data}

The one-step linear GN solver may be applied to weighted average data in order to implement ensemble averaging. Given a temporal window with a half width $d$, we model the time constant, $\tau$, to represent the rate at which the most rapid changes of interest occur in the body. That means that a feature of interest in a frame will dissipate by a factor of $\gamma=\exp \left(-t_{F} / \tau\right)$ in the next frame, and by $\gamma^{d}$ in the $d^{t h}$ subsequent frame. Using this factor, we calculate a weighted ensemble average EIT measurement, $\overline{\mathbf{y}}$

$$
\overline{\mathbf{y}}=\frac{1}{w_{\gamma}} \sum_{i=-d}^{d} \gamma^{|i|} \mathbf{y}_{i}
$$

where $w_{\gamma}=\sum_{i=-d}^{d} \gamma^{|i|}$. When $0<\gamma<1$, this is a forgetting process, and when $\gamma=1$, an averaging process. Noise amplitude will decrease by a factor of $\sqrt{w_{\gamma}}$ due to this ensemble averaging. The GN solver with weighted data reconstructs images as

$$
\hat{\mathbf{x}}=\mathbf{B} \overline{\mathbf{y}}
$$

where $\mathbf{B}$ is calculated using (3.4).

\subsubsection{Kalman solver}

The Kalman filter is a widely used approach for many tracking and data prediction tasks. The EIT image reconstruction algorithm of Vauhkonen et al. (1998a) is formulated as an iterative state estimation problem. The system discrete time prediction model is given by (using the notation introduced above)

$$
\mathbf{x}_{k}=\mathbf{A} \mathbf{x}_{k-1}+\mathbf{v}
$$

for a discrete time sequence $k . \mathbf{A} \in \mathbb{R}^{n_{N} \times n_{N}}$ is the state transition matrix, and $\mathbf{v} \in \mathbb{R}^{n_{N}}$ is the state noise (assumed to be zero mean white Gaussian). One traditional difficulty with 
Kalman filters is finding values for state space parameters. If the underlying processes in the body were well known, then A could be derived; for example, in a stirred mixing tank, the process would rotate image elements in each frame. We follow the traditional heuristic approach of assigning $\mathbf{A}=\mathbf{I}$ (representing a random walk process) and setting the state noise covariance to the image element covariance $\mathbf{P}$. The discrete time observation model at time step $k$ is:

$$
\mathbf{y}_{k}=\mathbf{J}_{k} \mathbf{x}_{k}+\mathbf{n}
$$

which is equivalent to (3.1) if $\mathbf{J}$ is constant. Kalman image reconstruction iteratively estimates $\mathbf{x}_{k}$ based on the previous image $\mathbf{x}_{k-1}$ and measurements $\mathbf{y}_{k}$.

$$
\begin{aligned}
\mathbf{x}_{k}^{-} & =\mathbf{A} \hat{\mathbf{x}}_{k-1} \quad \text { state estimation } \\
\hat{\mathbf{x}}_{k} & =\mathbf{x}_{k}^{-}+\mathbf{K}_{k}\left(\mathbf{y}_{k}-\mathbf{J}_{k} \mathbf{x}_{k}^{-}\right) \quad \text { state correction }
\end{aligned}
$$

where $\mathbf{K}_{k}$ is the Kalman gain, which is calculated from the error covariance estimate $\mathbf{C}_{k}$ as:

$$
\begin{aligned}
\mathbf{C}_{k}^{-} & =\mathbf{A} \hat{\mathbf{C}}_{k-1} \mathbf{A}^{T}+\mathbf{P} \text { error covariance estimation } \\
\mathbf{K}_{k} & =\mathbf{C}_{k}^{-} \mathbf{J}_{k}^{T}\left(\mathbf{J}_{k} \mathbf{C}_{k}^{-} \mathbf{J}_{k}^{T}+\mathbf{V}\right)^{-1} \quad \text { Kalman gain } \\
\hat{\mathbf{C}}_{k} & =\left(\mathbf{I}-\mathbf{K}_{k} \mathbf{J}_{k}\right) \mathbf{C}_{k}^{-} \quad \text { error covariance correction }
\end{aligned}
$$

Iterative calculation of $\mathbf{K}$ is computationally expensive. If $\mathbf{J}$ is constant, $\mathbf{K}$ will eventually stabilize, and may be precomputed (Kim et al., 2006) in order to dramatically speed up the calculation. We do not take this approach here.

\subsubsection{Temporal one-step solver}

Instead of calculating an image based on the sequence of past frames, we propose a temporal image reconstruction algorithm which uses a set of data frames nearby in time. The data frame sequence is treated as a single inverse problem, with a regularization prior to account for both spatial and temporal correlations between image elements. Given a vertically concatenated sequence of measurements frames $\tilde{\mathbf{y}}_{t}=\left[\mathbf{y}_{t-d} ; \ldots ; \mathbf{y}_{t} ; \ldots ; \mathbf{y}_{t+d}\right]$ and the corresponding concatenated images $\tilde{\mathbf{x}}_{t}=\left[\mathbf{x}_{t-d} ; \ldots ; \mathbf{x}_{t} ; \ldots ; \mathbf{x}_{t+d}\right]$, the direct temporal 
forward model (3.1) is rewritten as

$$
\left[\begin{array}{c}
\mathbf{y}_{t-d} \\
\vdots \\
\mathbf{y}_{t} \\
\vdots \\
\mathbf{y}_{t+d}
\end{array}\right]=\left[\begin{array}{ccccc}
\mathbf{J} & & \cdots & & 0 \\
& \ddots & & & \\
\vdots & & \mathbf{J} & & \vdots \\
& & & \ddots & \\
0 & & \cdots & & \mathbf{J}
\end{array}\right]\left[\begin{array}{c}
\mathbf{x}_{t-d} \\
\vdots \\
\mathbf{x}_{t} \\
\vdots \\
\mathbf{x}_{t+d}
\end{array}\right]+\left[\begin{array}{c}
\mathbf{n}_{t-d} \\
\vdots \\
\mathbf{n}_{t} \\
\vdots \\
\mathbf{n}_{t+d}
\end{array}\right]
$$

and also as

$$
\tilde{\mathbf{y}}_{t}=\tilde{\mathbf{J}} \tilde{\mathbf{x}}_{t}+\tilde{\mathbf{n}}_{t}
$$

where $\tilde{\mathbf{n}}_{t}=\left[\mathbf{n}_{t-d} ; \ldots ; \mathbf{n}_{t} ; \ldots ; \mathbf{n}_{t+d}\right]$. We assume $\mathbf{J}$ to be constant, although this formulation could be modified to account for a time varying $\mathbf{J}$. Based on this approximation $\tilde{\mathbf{J}}=\mathbf{I} \otimes \mathbf{J}$, where the identity matrix I has size $2 d+1$, and $\otimes$ is the Kronecker product.

The correlation of corresponding elements between adjacent frames (delay $\delta=1$ ) can be represented by an inter-frame correlation $\gamma$ which has value between 0 (independent) and 1 (fully dependent). As frames become separated in time, the inter-frame correlation decreases; for an inter-frame separation $\delta$, the inter-frame correlation is $\gamma^{\delta} . \gamma$ could also possibly be negative if subsequent frames have inverse correlation, although this scenario is physiologically unrealistic. Frames with large inter-frame delay, $|\delta|>d$, are considered independent. Image reconstruction is then defined in terms of minimizing the augmented expression:

$$
\left\|\left[\begin{array}{c}
\mathbf{y}_{t-d} \\
\vdots \\
\mathbf{y}_{t} \\
\vdots \\
\mathbf{y}_{t+d}
\end{array}\right]-\left[\begin{array}{ccccc}
\mathbf{J} & & \ldots & & 0 \\
& \ddots & & & \\
\vdots & & \mathbf{J} & & \vdots \\
& & & \ddots & \\
0 & & \ldots & & \mathbf{J}
\end{array}\right]\left[\begin{array}{c}
\mathbf{x}_{t-d} \\
\vdots \\
\mathbf{x}_{t} \\
\vdots \\
\mathbf{x}_{t+d}
\end{array}\right]\right\|_{\tilde{\mathbf{W}}}
$$

and (3.5) becomes

$$
\tilde{\mathbf{B}}=\tilde{\mathbf{R}}^{-1} \tilde{\mathbf{J}}^{T}\left(\tilde{\mathbf{J}} \tilde{\mathbf{R}}^{-1} \tilde{\mathbf{J}}^{T}+\lambda^{2} \tilde{\mathbf{W}}^{-1}\right)^{-1}
$$

where $\tilde{\mathbf{W}}=\mathbf{I} \otimes \mathbf{W} . \tilde{\mathbf{W}}$ is diagonal since measurement noise is uncorrelated between frames. $\tilde{\mathbf{R}}=\Gamma^{-1} \otimes \mathbf{R}$ where $\boldsymbol{\Gamma}$ is the temporal weight matrix of an image sequence $\tilde{\mathbf{x}}$ and is defined 
to have the form as

$$
\boldsymbol{\Gamma}=\left[\begin{array}{ccccc}
1 & \gamma & \ldots & \gamma^{2 d-1} & \gamma^{2 d} \\
\gamma & 1 & \ldots & \gamma^{2 d-2} & \gamma^{2 d-1} \\
\vdots & \vdots & \ddots & \vdots & \vdots \\
\gamma^{2 d-1} & \gamma^{2 d-2} & \ldots & 1 & \gamma \\
\gamma^{2 d} & \gamma^{2 d-1} & \ldots & \gamma & 1
\end{array}\right]
$$

From (3.18) and (3.19),

$$
\tilde{\mathbf{B}}=\left[\boldsymbol{\Gamma} \otimes\left(\mathbf{P} \mathbf{J}^{T}\right)\right]\left[\boldsymbol{\Gamma} \otimes\left(\mathbf{J P} \mathbf{J}^{T}\right)+\lambda^{2}(\mathbf{I} \otimes \mathbf{V})\right]^{-\mathbf{1}}
$$

Thus, (3.4) is rewritten as

$$
\left[\begin{array}{c}
\hat{\mathbf{x}}_{t-d} \\
\vdots \\
\hat{\mathbf{x}}_{t} \\
\vdots \\
\hat{\mathbf{x}}_{t+d}
\end{array}\right]=\tilde{\mathbf{B}} \tilde{\mathbf{y}}_{t}
$$

Although this estimate is an augmented image sequence, we are typically only interested in the current image $\hat{\mathbf{x}}_{t}$. which is calculated from $\hat{\mathbf{x}}_{t}=\tilde{\mathbf{B}}_{d} \tilde{\mathbf{y}}_{t}$ where $\tilde{\mathbf{B}}_{d}$ is the rows $n_{M} d+$ $1 \ldots n_{M}(d+1)$ of $\tilde{\mathbf{B}}$.

\subsubsection{Noise figure}

In order to compare the different image reconstruction algorithms, it is important to choose corresponding values of the hyperparameter for each algorithm. For a review of hyperparameter selection methods for EIT, refer to Graham and Adler (2006). For our application, we wish to compare the resolution and noise performance across algorithms; however, since the regularization hyperparameter implicitly controls the compromise between resolution and noise performance, we choose to control for noise performance across algorithms, and then compare the resolution. Image reconstruction noise performance may be measured with the noise figure (NF) parameter of Adler and Guardo (1996a). Here we generalize the NF calculation to apply to any difference EIT formulation, not simply one-step Gauss Newton type algorithms.

An EIT difference measurement vector is $\mathbf{y}=\mathbf{y}_{0}+\mathbf{n}_{y}$, where $\mathbf{y}_{0}$ is the deterministic underlying signal, and $\mathbf{n}_{y}$ is stochastic, zero mean, measurement noise. Typically, components of $\mathbf{n}_{y}$ are independent, but this formulation does not make this assumption. For difference EIT, $[\mathbf{y}]_{i}=\left[\mathbf{v}_{2}\right]_{i}-\left[\mathbf{v}_{\mathbf{1}}\right]_{i}$, and components of $\mathbf{n}_{y}$ are often assumed to be equal, 
but this may potentially vary if the gain varies between channels. For normalized difference EIT, $[\mathbf{y}]_{i}=\left(\left[\mathbf{v}_{2}\right]_{i}-\left[\mathbf{v}_{1}\right]_{i}\right) /\left[\mathbf{v}_{1}\right]_{i}$, and components of $\mathbf{n}_{y}$ are scaled by $\operatorname{diag}\left(\mathbf{v}_{1}\right)^{-1}$. Both $\mathbf{y}_{0}$ and $\mathbf{n}_{y}$ may be complex valued.

The signal to noise ratio (SNR) of the difference measurement is defined as:

$$
\mathrm{SNR}_{y}=\frac{E[|\mathbf{y}|]}{\sqrt{\operatorname{var}(\mathbf{y})}}
$$

where we approximate $E[|\mathbf{y}|]=\frac{1}{n_{M}} \sum\left|\mathbf{y}_{0}\right|$ and calculate $\operatorname{var}(\mathbf{y})=\frac{1}{n_{M}} \operatorname{trace} \boldsymbol{\Sigma}_{n}$ where $\boldsymbol{\Sigma}_{n}$ is the measurement noise covariance. $n_{M}$ is the number of measured values in the EIT data frame; we divide by $n_{M}$ rather than $n_{M}-1$ to calculate the variance, since the noise is known to be zero mean.

This covariance may be modelled by a noise basis, $\mathbf{N}_{y}$ such that $\mathbf{N}_{y} \mathbf{N}_{y}^{T}=\boldsymbol{\Sigma}_{n}$. For difference EIT with independent noise on each channel, this is a diagonal matrix with $\left[\mathbf{N}_{y}\right]_{i, i}$ equal to the noise amplitude on channel $i$. Using this noise basis, $\operatorname{var}(\mathbf{y})=\frac{1}{n_{M}}\left\|\mathbf{N}_{y}\right\|_{F}^{2}$, where $\|\cdot\|_{F}^{2}$ is the sum of each matrix element squared (Frobenius norm squared).

A general EIT reconstruction algorithm, EIT, reconstructs an image estimate, $\hat{\mathbf{x}}$ from measurements as $\hat{\mathbf{x}}=\operatorname{EIT}(\mathbf{y})$. This notation is also extended to reconstruct a matrix of column concatenated images independently from a matrix of measurements. The signal to noise ratio (SNR) of the difference image is:

$$
\operatorname{SNR}_{x}=\frac{E[|\mathbf{x}|]}{\sqrt{\operatorname{var}(\mathbf{x})}}
$$

where we approximate $E[|\mathbf{x}|]=\sum \mathbf{A}\left|\mathbf{x}_{0}\right|$ where $\mathbf{x}_{0}=\operatorname{EIT}\left(\mathbf{y}_{0}\right)$ and $\mathbf{A}$ is a diagonal matrix of the volume (or area in 2D) of each reconstructed image element. We calculate $\operatorname{var}(\mathbf{x})=$ trace $\mathbf{A}^{2} \boldsymbol{\Sigma}_{x}$ where $\boldsymbol{\Sigma}_{x}$ is the image noise covariance. For difference EIT, image reconstruction is linear for small $\mathbf{y}$, Using noise basis, $\mathbf{N}_{x}$, we calculate $\boldsymbol{\Sigma}_{x}=\mathbf{N}_{x} \mathbf{N}_{x}^{T}$ where $\mathbf{N}_{x}=\operatorname{EIT}\left(\mathbf{N}_{y}\right)$ for small $\mathbf{N}_{y}$, and $\operatorname{var}(\mathbf{x})=\left\|\mathbf{A N}_{x}\right\|_{F}^{2}$.

The NF is the ratio of output to input SNR, where the input signal $\mathbf{y}_{0}$ is chosen to be a small change in a central inner circular disk covering $10 \%$ of the medium diameter, and $\mathbf{N}_{n}$ is scaled to be within the linear range of the algorithm.

$$
\mathrm{NF}=\frac{\mathrm{SNR}_{x}}{\mathrm{SNR}_{z}}=\frac{\sum \mathbf{A}\left|E I T\left(\mathbf{y}_{0}\right)\right|}{\sum\left|\mathbf{y}_{0}\right|} \sqrt{\frac{\left\|n_{M} \mathbf{N}_{y}\right\|_{F}^{2}}{\left\|\mathbf{A} E I T\left(\mathbf{N}_{y}\right)\right\|_{F}^{2}}}
$$

While the SNR is normally defined in terms of the signal power, here we define it in terms of absolute amplitude. This is necessary because it is the signal amplitude, and 
not the power, that is spread across image elements with changes in hyperparameter; our experiments with the signal power definition do not show stable or useful results. Finally, in distinction to the definition in Adler and Guardo (1996a), we calculate the absolute amplitude of the signal, allowing this definition to be appropriate to EIT systems which measure complex signals.

\subsection{Results}

Numerical simulations were conducted using a planar 2D FEM model with 5184 elements using the EIDORS software (Adler and Lionheart, 2006). A unit radius circular medium with 16 electrodes using adjacent stimulation and measurement pattern is simulated, in which a non-conductive spherical object with 0.05 unit radius rotates clockwise along a trajectory that has a radius of $2 / 3$ unit, moving at a speed of one rotation per 40 frames. The noise performance of the algorithms was tested by adding pseudo random, zero mean Gaussian noise. All reconstructed images in figure 3.1 and 3.2 used the same random seed; tests with different seed values did not vary significantly. Images were reconstructed on a 576 element mesh, which differs from the simulation model to avoid the inverse crime.

Reconstructed images were calculated for four image algorithms and are shown in corresponding columns in Figures 3.1 and 3.2: 1) Gauss-Newton, 2) GN with weighted data, 3) temporal solver and 4) Kalman filter. In each image, the position of the target at all data frames used in the algorithms are shown. In all cases, the target was at $(x, y)=\left(-\frac{2}{3}, 0\right)$ in the image shown. We explored the behaviour of these algorithms as a function of regularization hyperparameter for both noise free and noisy data. In order to choose a hyperparameter to allow comparison across algorithms, we select its value for each algorithm in order to give a fixed $N F$ value (section 3.3.5). Figure 3.2 shows reconstructed images for a low hyperparameter value (giving $N F=2.0$ ), while figure 3.1 shows images for a higher value (giving $N F=0.1$ ). Noise levels were chosen heuristically in order to illustrate the algorithm noise performance.

\subsection{Discussion}

Traditionally, EIT reconstruction algorithms assume each data frame to be independent. However, since EIT is able to make measurements at high frame rates, we know a priori that image frames are correlated. This chapter addresses reconstruction of EIT data for 


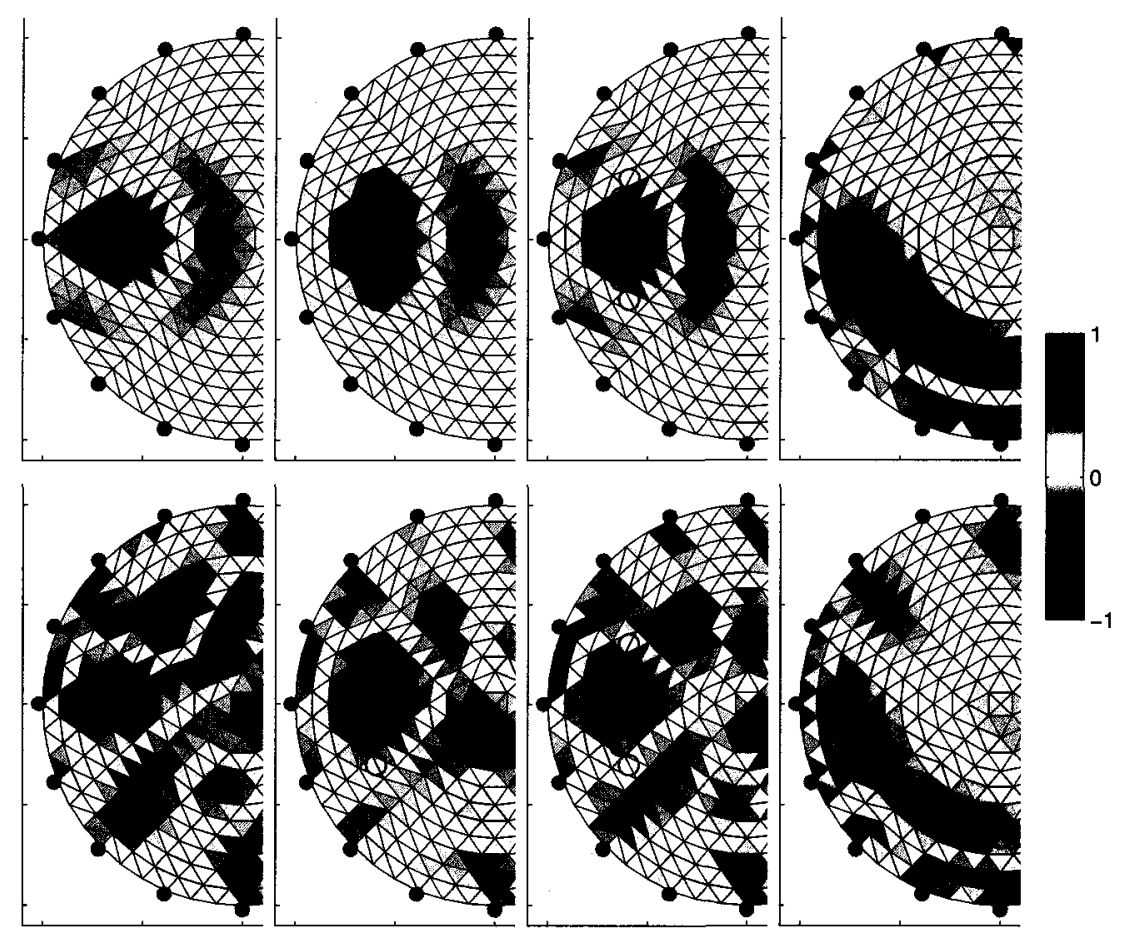

Figure 3.1: Reconstructed images of a target at $\left(-\frac{2}{3}, 0\right)$ for high hyperparameter (parameters $N F=0.1, \gamma=0.8$ and $d=3$ ). Top row: No noise; Bottom row: $S N R=0.25$ Each column uses a different reconstruction algorithm: $A$ : Gauss-Newton $B$ : Gauss-Newton with weighted data $C$ : Temporal Solver $D$ : Kalman Filter. The black circles in the images indicate the position of the simulated target in each data frame used for in the image reconstruction. The colourbar (with normalized units) is shown at right. 

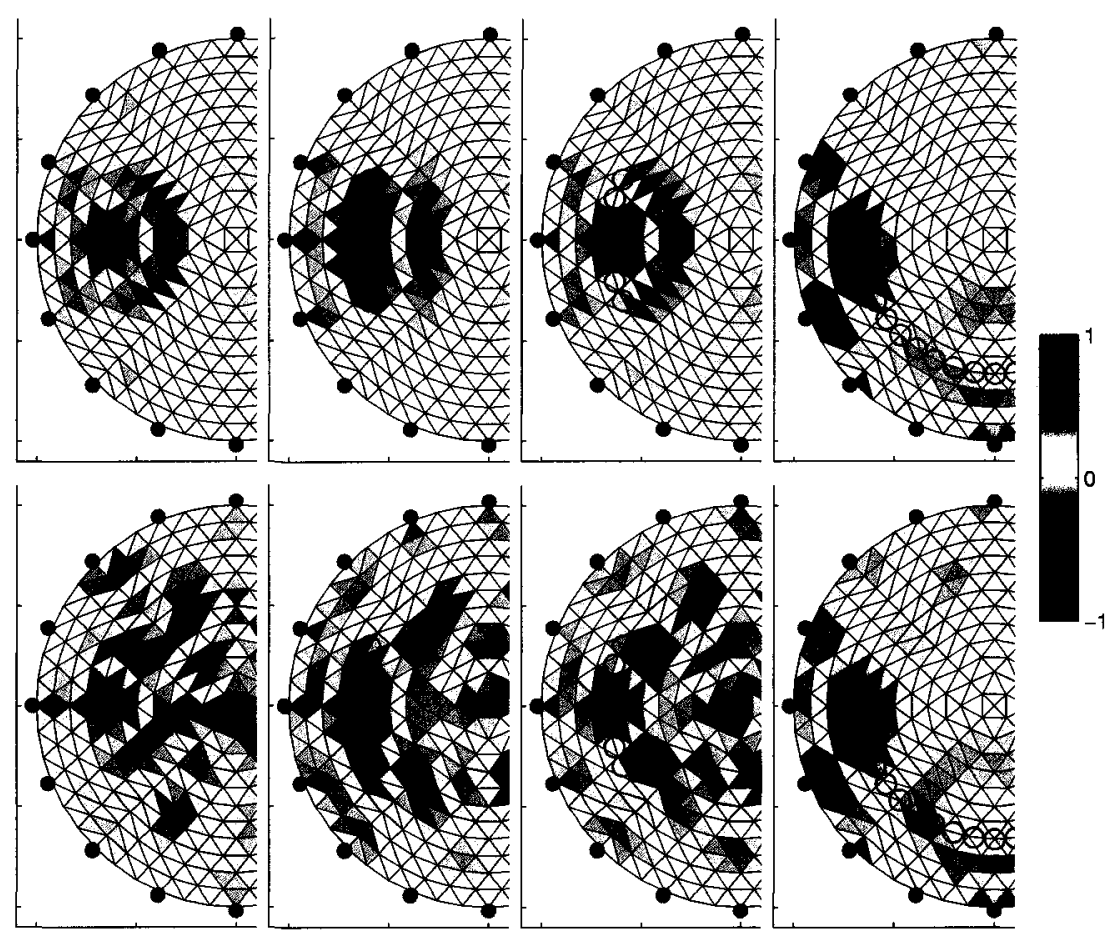

Figure 3.2: Reconstructed images of a target at $\left(-\frac{2}{3}, 0\right)$ for low hyperparameter (chosen for $N F=2.0, \gamma=0.8$ and $d=3$ ). Top row: No noise; Bottom row: $S N R=4.0$ Each column uses a different reconstruction algorithm: $A$ : Gauss-Newton $B$ : Gauss-Newton with weighted data $C$ : Temporal Solver $D$ : Kalman Filter. The black circles in the images indicate the position of the simulated target in each data frame used for in the image reconstruction. The colourbar (with normalized units) is shown at right. 
temporal reconstructions, in which we use temporal correlations to improve reconstructed image SNR. A new temporal reconstruction algorithm is introduced, which directly formulates the temporal inverse in terms of a single regularized expression. We compare four different algorithms: one-step $G N$ (with no temporal behaviour), $G N$ with weighted data, Kalman filter reconstruction, and the proposed temporal reconstruction.

Results show that the $G N$ algorithm is able to accurately reconstruct the position and shape of the target, but shows poorer noise performance than the other algorithms. As the hyperparameter increases (figure 3.1), GN images tend to image targets closer toward the centre of the body. This effect is well understood for EIT (Adler and Guardo, 1996a), and is probably a consequence of the prior weighting of central image elements. The $G N$ with averaged data blurs the reconstructed image across all the averaged data frames, but is able to show improved noise performance, as is expected from ensemble averaging. Thus, $G N$ algorithms are recommended when noise levels are low, and $G N$ with weighted data is a good solution when the conductivity is changing slowly with respect to the frame rate.

At low hyperparameter, the Kalman filter tends to reconstruct images with the target "pushed" outward toward the boundary, and create image artifacts and image noise on the boundary. We hypothesize that this effect is due to the iterative calculation of the error covariance term (in (3.12)), which results in a $\mathbf{C}^{-}$which tends toward the identity matrix. In the Kalman formulation, this term takes the place of $\mathbf{R}$ in the $G N$ inverse, making the Kalman filter images resemble $G N$ images with zero-order Tikhonov regularization. For larger hyperparameter values (figure 3.1), Kalman filter images tend to show a "trail" as a larger weighting is given to previous frame data in the current image calculation. The temporal regularization method can in some circumstances introduce a "trail" which enlarges the reconstructed image to include recent future and past target positions. This can occur when the inter-frame correlation value, $\gamma$, is chosen unreasonably high. In this case, the current image is made wrongly dependent on adjacent images.

At high hyperparameter values, the temporal reconstruction shows improved resolution (illustrated as figure 3.1). While at low hyperparameter, it gives similar images to that of $G N$ (illustrated as figure 3.2). This behaviour may be understood, since at low hyperparameter $(\lambda \approx 0),(3.20)$ approximates

$$
\tilde{\mathbf{B}} \approx\left[\boldsymbol{\Gamma} \otimes\left(\mathbf{P J} \mathbf{J}^{T}\right)\right]\left[\mathbf{\Gamma} \otimes\left(\mathbf{J P J} \mathbf{J}^{T}\right)\right]^{-1}=\mathbf{I} \otimes\left[\mathbf{P} \mathbf{J}^{T}\left(\mathbf{J P J} \mathbf{J}^{T}\right)^{-1}\right]
$$

which will reconstruct each data frame independently. On the other hand, at high hyperparameter, the reconstructed image will weigh data frames together, as per $G N$ with 
weighted data,

$$
\tilde{\mathbf{B}} \approx\left[\mathbf{\Gamma} \otimes\left(\mathbf{P} \mathbf{J}^{T}\right)\right]\left[\lambda^{2}(\mathbf{I} \otimes \mathbf{V})\right]^{-1}=\mathbf{\Gamma} \otimes\left[\mathbf{P J}^{T}\left(\lambda^{2} \mathbf{V}\right)^{-1}\right]
$$

In this chapter, the temporal weight $\gamma$ is chosen heuristically; however, objective selection of $\gamma$ is possible; its value could be estimated from the covariance of data frames measured.

In summary, this chapter proposes a temporal EIT reconstruction algorithm. For low noise solutions (low hyperparameter) its behaviour is approximates that of Gauss-Newton reconstruction, while for high noise level and high frame rates cases where large hyperparameters are adopted, it is advantageous by reconstructing higher resolution images. It improves over Kalman filter based algorithms by allowing an explicit control over the regularization prior and the weighting of measured data. We recommend the temporal algorithm for cases in which the data noise is high and the underlying conductivity changes are slow with respect to the frame rate. 


\section{Chapter 4}

\section{EIT Image Reconstruction with Four Dimensional Regularization}

\subsection{Summary}

This chapter is based on the paper -EIT Image Reconstruction with Four Dimensional Regularization, Medical and Biological Engineering and Computing, In press, by Tao Dai, Manuchehr Soleimani and Andy Adler, 2008.

Electrical impedance tomography (EIT) reconstructs internal impedance images of the body from electrical measurements on body surface. The temporal resolution of EIT data can be very high, although the spatial resolution of the images is relatively low. Most EIT reconstruction algorithms calculate images from data frames independently, although data are actually highly correlated especially in high speed EIT systems. This chapter proposes a 4-D EIT image reconstruction for functional EIT. The new approach is developed to directly use prior models of the temporal correlations among images and 3-D spatial correlations among image elements. A fast algorithm is also developed to reconstruct the regularized images. Image reconstruction is posed in terms of an augmented image and measurement vector which are concatenated from a specific number of previous and future frames. The reconstruction is then based on an augmented regularization matrix which reflects the $a$ priori constraints on temporal and 3-D spatial correlations of image elements. A temporal factor reflecting the relative strength of the image correlation is objectively calculated from measurement data. Results show that image reconstruction models which account for inter-element correlations, in both space and time, show improved resolution and noise performance, in comparison to simpler image models. 


\subsection{Introduction}

This chapter develops a regularized image reconstruction formulation which directly accounts for temporal and spatial correlations between image elements in Electrical Impedance Tomography (EIT). EIT reconstructs images of the impedance distribution within the body from electrical measurement on the body surface. Because electrical current propagates diffusely in the body, EIT is a soft field tomography modality; moreover, EIT is ill-posed according to conditions of well-posed problems suggested by Hadamard. Thus, EIT reconstruction has poor spatial resolution and is very sensitive to data errors (Lionheart et al., 2005). However, EIT can have excellent temporal resolution (frame rates as high as 1000 frames/s have been achieved (Wilkinson et al., 2005)) which makes EIT an attractive modality to monitor fast physiological activities which produce conductivity distribution changes. This is valuable for monitoring of cardiac (Eyuboglu et al., 1989; Vonk-Noordegraaf et al., 2000), pulmonary (Adler et al., 1996b; Frerichs, 2000; Harris et al., 1992) and brain (Holder, 1992) activities. The normal heart rate is physiologically limited to about $4 \mathrm{~Hz}$, while a fibrillating heart rate can beat at up to $8 \mathrm{~Hz}$ (Bollmann et al., 1998). The frequency content of images can be much higher than the heart rate as shown by the frequency content of the QRS complex which is mainly between $10-25 \mathrm{~Hz}$ (Kohler et al., 2002). For lung monitoring, high frequency ventilation (at frequencies of $5-25 \mathrm{~Hz}$ ) is understood to be helpful to patients with respiratory distress; these patients have highly a non-uniform distribution of ventilation in the lung (van Genderingen et al., 2004) and monitoring with EIT has been shown to provide clinically useful information for control of ventilator settings (Wolf and Arnold, 2005). Finally, EIT is potentially useful for monitoring brain activity. Tests such as visually evoked responses or monitoring for epilepsy result in rapid conductivity variations (Tidswell et al., 2001).

EIT uses single or multi-plane electrode arrangements to inject stimulation currents and measure the voltage response (Dehgani et al., 2005; Metherall et al., 1996; Graham and Adler, 2007). It is limited by the relatively small number of attached electrodes, and the severe ill-conditioning of the inverse problem due to the surface measurements and diffuse nature of electrical stimulation. In order to calculate a "reasonable" image, regularization techniques are required. Such regularized image reconstruction can be statistically formulated, in terms of a priori information, as a prior matrix describing image element values and the correlation between them. In many EIT algorithms, the zeroth order Tikhonov priors are commonly used (Cheney et al., 1990; Vauhkonen et al., 1999). While such priors are easy to compute, they assume that elements of the conductivity distributions are statistically 
independent - which is clearly not true for most EIT applications. Previously, shape-based inverse solutions were investigated in EIT applications. These methods modelled spatial priors according to geometrical knowledge of the target surface. e.g., boundary element method (BEM) based 3-D solution (Babaeizadeh and Brooks, 2007), and spline-based 2-D solution (Tossavainen et al., 2004).

Another limitation to the quality of EIT images is signal noise. In cases where the physiological changes are slow with respect to the frame rate, time averaging of frames may be used to reduce noise by the square root of the number of averaged frames, given uncorrelated noise. However, if EIT data acquisition rate is comparable to the rate of change in the imaged processes, time averaging sacrifices the temporal resolution. Some other signal uncertainties also introduce image degradations, e.g., electrode-skin contact impedance drift which causes image distortion over time (Boone and Holder, 1996). In these cases, each frame of EIT data is typically reconstructed independently of the others. However, it is clear that individual data frames are not completely independent, but do contain useful temporal correlations which could be exploited to improve EIT image noise performance and resolution.

Temporal image reconstruction can be represented as a linear tracking problem. In (Schmitt and Louis, 2002; Schmitt et al., 2002), a priori information about "temporal smoothness" was considered by adding one term which measures the variation between adjacent images, into the Tikhonov-Phillips minimization task. Another temporal reconstruction method in EIT is the Kalman filter (Vauhkonen et al., 1998a; Seppanen et al., 2001), in which the image at each instant is estimated from the current data and the previous image estimate.

In this chapter, we make the novel contribution of a general approach to model the 4-D (temporal and 3-D spatial) correlations in the regularization prior. As image elements move further apart in space and time, the correlation between them is modelled to decrease exponentially, with exponential constants $\eta$ and $\gamma$ in space and time, respectively. This approach reconstructs each 3-D image at frame $t$ using the set of data in frames $t-d$ to $t+d$, where $d$ is the frame window width. Next, in order to reduce the computational burden of such large models, we develop an efficient formulation of the inverse matrices. This chapter is an extension of our conference publication (Dai et al., 2007), where we considered only the vertical inter-slice correlation. In the results section, we show that such 4-D prior modelling gives improvements in both resolution and noise performance in the reconstructed images. 


\subsection{Methods}

We consider an EIT system with $n_{E}$ electrodes placed in 2 rings on body surface using planar placement (Graham and Adler, 2007) and adjacent current stimulation with parallel voltage measurement. $n_{E}$ current stimulation patterns are sequentially applied and $n_{V}$ differential measurements are made for each stimulation. We do not measure the voltages on the stimulation electrodes (however, given the proper hardware, such measurements are recommended (Babaeizadeh and Brooks, 2006)), thus $n_{V}=n_{E}-3$ and the total number of measurements within one data frame is $n_{M}=n_{E} \times\left(n_{E}-3\right)$. Difference EIT calculates difference signal $\mathbf{y} \in \mathbb{R}^{n_{M}}, \mathbf{y}_{i}=\mathbf{v}_{i}-\left[\mathbf{v}_{0}\right]_{i}$, or normalized as $\mathbf{y}_{i}=\left(\mathbf{v}_{i}-\left[\mathbf{v}_{0}\right]_{i}\right) /\left[\mathbf{v}_{0}\right]_{i}$, where the subscript $i$ represents the measurement sequence. $\left[\mathbf{v}_{0}\right]_{i}$ is the reference measurement, obtained at a time when conductivity is more stable (such as diastole during a breath hold). In real applications, $\mathbf{v}_{0}$ can be obtained by averaging over several cycles of ventilation.

The body under investigation is modelled as a cylindrical Finite Element Model (FEM) with $n_{N}$ piecewise smooth tetrahedral elements, represented by a vector $\sigma \in \mathbb{R}^{n_{N}}$ ( $\sigma$ represents conductivity in this paragraph, elsewhere in this chapter, $\sigma$ is the standard deviation). Difference EIT calculates a vector of conductivity change, $\mathbf{x}_{i}=\sigma_{i}-\left[\sigma_{0}\right]_{i}$ between the present conductivity distribution, $\sigma$, and the reference conductivity distribution, $\sigma_{0}$, which is typically assumed to be homogenous (in this chapter, $\sigma_{0}=1 \mathrm{~S} / \mathrm{m}$ ). For small variations around $\sigma_{0}$, the relationship between the conductivity change image $\mathbf{x}_{t}$ and difference measurements $\mathbf{y}_{t}$ at time $t$ can be linearized (giving the difference EIT forward model):

$$
\mathbf{y}_{t}=\mathbf{J} \mathbf{x}_{t}+\mathbf{n}
$$

where $\mathbf{J} \in \mathbb{R}^{n_{M} \times n_{N}}$ is the Jacobian or sensitivity matrix; $\mathbf{n} \in \mathbb{R}^{n_{M}}$ is the measurement noise which is assumed to be uncorrelated white Gaussian. $\mathbf{J}$ is calculated from the FEM

as $\mathbf{J}_{i j}=\left.\frac{\partial \mathbf{y}_{i}}{\partial \mathbf{x}_{j}}\right|_{\sigma_{0}}$. The goal of regularized image reconstruction (such as Cheney et al. 1990; Adler and Lionheart 2006) is to calculate a conductivity change estimate, $\hat{\mathbf{x}}_{t}$, which is both faithful to the measurements, $\mathbf{y}_{t}$, and to a priori constraints on a "reasonable" image.

In subsequent sections we develop a formulation for the spatial and temporal a priori image element correlations, and use these to consider the following reconstruction approaches: 1) Gauss-Newton (GN) inverse, using the current measurement frame $\mathbf{y}_{t}$ only; 2) Temporal inverse, using measurement frames $\mathbf{y}_{t-d} \ldots \mathbf{y}_{t+d}$ based on a temporal prior model; 3) 3-D spatial inverse, using measurement $\mathbf{y}_{t}$ based on a 3-D spatial prior model with spatial correlations among elements; and 4) 4-D prior inverse, using measurement $\mathbf{y}_{t-d} \ldots \mathbf{y}_{t+d}$ and a temporal and and 3-D spatial model. 


\subsubsection{One-step linear Gauss-Newton solver}

Regularized image reconstruction for EIT based on the one-step linearized Gauss-Newton (GN) solver was first introduced into EIT by (Yorkey et al., 1987), and has been widely used. It calculates a linear reconstruction matrix which may subsequently be used for rapid imaging, and allows taking advantage of sophisticated regularization modalities to solve the inverse problem. This method seeks a solution, $\hat{\mathbf{x}}$, which minimizes the error in the form

$$
\|\mathbf{y}-\mathbf{J} \hat{\mathbf{x}}\|_{\Sigma_{n}^{-1}}^{2}+\left\|\hat{\mathbf{x}}-\mathbf{x}^{\circ}\right\|_{\Sigma_{x}^{-1}}^{2}
$$

here $\mathbf{x}^{\circ}$ is the expected value of element conductivity changes, which is zero for difference EIT since it assumes that the conductivity changes may be equally positive or negative. $\boldsymbol{\Sigma}_{n} \in \mathbb{R}^{n_{M} \times n_{M}}$ is the covariance matrix of the measurement noise $\mathbf{n}$. Since $\mathbf{n}$ is uncorrelated, $\boldsymbol{\Sigma}_{n}$ is a diagonal matrix with $\left[\boldsymbol{\Sigma}_{n}\right]_{i, i}=\sigma_{i}^{2}$, where $\sigma_{i}^{2}$ is the noise variance at measurement $i$. $\Sigma_{x} \in \mathbb{R}^{n_{N} \times n_{N}}$ is the covariance matrix of the expected image, which we consider in detail subsequently.

Instead of calculating $\boldsymbol{\Sigma}_{n}$ and $\boldsymbol{\Sigma}_{x}$ directly, most proposed approaches have developed models of the inverse of these matrices heuristically. We use the following terminology: $\mathbf{W}=\sigma_{n}^{2} \boldsymbol{\Sigma}_{n}^{-1}$ and $\mathbf{R}=\sigma_{x}^{2} \boldsymbol{\Sigma}_{x}^{-1}$, where $\sigma_{n}$ is the average measurement noise amplitude and $\sigma_{x}$ is the a priori amplitude of conductivity changes. $\mathbf{W}$ models the relative measurement accuracy across channels. For uncorrelated noise, each diagonal element is proportional to the signal-to-noise-ratio (SNR). For difference EIT with identical channels, $\mathbf{W}$ is an identity matrix; in this chapter $\mathbf{W}=\mathbf{I}$. The regularization matrix $\mathbf{R}$ may be understood to model the "unlikelihood" of image element configurations. A simple model for $\mathbf{R}$ may consider all configurations equally likely, while more sophisticated models, such as the ones we develop, may consider smooth distributions more likely than rapidly changing ones.

By solving (4.2) and defining the hyperparameter $\lambda=\sigma_{n} / \sigma_{x}$, a linearized, one-step inverse solution is obtained

$$
\hat{\mathbf{x}}=\left(\mathbf{J}^{T} \mathbf{W} \mathbf{J}+\lambda^{2} \mathbf{R}\right)^{-1} \mathbf{J}^{T} \mathbf{W} \mathbf{y}=\mathbf{B y}
$$

where $\mathbf{B}=\left(\mathbf{J}^{T} \mathbf{W} \mathbf{J}+\lambda^{2} \mathbf{R}\right)^{-1} \mathbf{J}^{T} \mathbf{W}$ is the linear, one-step reconstruction matrix. $\lambda$ controls the trade-off between resolution and noise attenuation in the reconstructed image.

By assuming that image elements are independent to each other and have identical expected magnitude, $\mathbf{R}$ becomes an identity matrix $\mathbf{I}$ and (4.3) uses zeroth-order Tikhonov regularization. For EIT, since the measured data is much more sensitive to boundary elements than elements deep inside, such solutions tend to push reconstructed noise toward 
the boundary. In order to compensate the sensitivity discrepancy, $\mathbf{R}$ may be scaled with the sensitivity of each element, so that $\mathbf{R}$ is a diagonal matrix with elements $[\mathbf{R}]_{i, i}=\left[\mathbf{J}^{T} \mathbf{J}\right]_{i, i}^{p}$. This is the NOSER prior of (Cheney et al., 1990) for an exponent $p$. One similar variance compensation strategy was proposed as variance uniformization in (Bacrie et al., 1997).

In (4.3), the term to be inverted is of size $n_{N} \times n_{N}$. This is especially cumbersome in $3 \mathrm{D}$ (and 4D) reconstruction models, where $n_{N}$ may be on the order of ten or a hundred thousand. This calculation is then very demanding on computer time and memory. We address this issue by rewriting the matrix $\mathbf{B}$ using the data form (also referred to as the Wiener filter form) (Adler et al., 2007) as:

$$
\mathbf{B}=\mathbf{P} \mathbf{J}^{T}\left(\mathbf{J P J} \mathbf{J}^{T}+\lambda^{2} \mathbf{V}\right)^{-1}
$$

where $\mathbf{P}=\mathbf{R}^{-1}=\boldsymbol{\Sigma}_{x} / \sigma_{x}^{2}$ and $\mathbf{V}=\mathbf{W}^{-1}=\boldsymbol{\Sigma}_{n} / \sigma_{n}^{2}$; Using (4.4), the size of the inverted matrix is significantly reduced to $n_{M} \times n_{M}$. This is especially helpful for large scale models, such as 3-D EIT models and the temporal inverse be introduced below.

\subsubsection{Temporal solver}

In this section, we develop a temporal image reconstruction algorithm which calculates the image at a current frame using a set of data frames nearby in time. This approach differs from Kalman filter based algorithms (Vauhkonen et al., 1998a) which estimate image $\mathbf{x}_{t}$ based on measurements $\mathbf{y}_{t}$ and the previous image estimate $\mathbf{x}_{t-1}$. The temporal solver treats the estimate of the image frame sequence as a single inverse problem, with a regularization prior which accounts for both spatial and temporal correlations between image elements.

\section{Temporal reconstruction}

The temporal solver considers a sequence of $2 d+1$ measurements frames from $t-d$ to $t+d$ around the current frame, $t$. Given a vertically concatenated sequence of measurements frames $\tilde{\mathbf{y}}_{t}$ and the corresponding concatenated images $\tilde{\mathbf{x}}_{t}$,

$$
\begin{aligned}
\tilde{\mathbf{y}}_{t} & =\left[\begin{array}{lllll}
\mathbf{y}_{t-d}^{T} & \ldots & \mathbf{y}_{t}^{T} & \ldots & \mathbf{y}_{t+d}^{T}
\end{array}\right]^{T} \\
\tilde{\mathbf{x}}_{t} & =\left[\begin{array}{lllll}
\mathbf{x}_{t-d}^{T} & \ldots & \mathbf{x}_{t}^{T} & \ldots & \mathbf{x}_{t+d}^{T}
\end{array}\right]^{T}
\end{aligned}
$$

the direct temporal forward model (4.1) is rewritten as

$$
\tilde{\mathbf{y}}_{t}=\tilde{\mathbf{J}} \tilde{\mathbf{x}}_{t}+\tilde{\mathbf{n}}
$$


where $\tilde{\mathbf{n}}=\left[\begin{array}{lllll}\mathbf{n}_{t-d}^{T} & \ldots & \mathbf{n}_{t}^{T} & \ldots & \mathbf{n}_{d}^{T}\end{array}\right]^{T}$. We consider that the model structure is constant, and thus $\mathbf{J}$ does not vary with time, giving $\tilde{\mathbf{J}}=\mathbf{I} \otimes \mathbf{J}$, where the identity matrix $\mathbf{I}$ has size $2 d+1$, and $\otimes$ is the Kronecker product.

The relationship between corresponding image elements between adjacent frames can be represented by an inter-frame correlation which has a value between 0 (independent) and 1 (fully dependent). The correlation could possibly be negative if subsequent frames have inverse correlation, although this scenario is physiologically unrealistic. As frames become separated in time, the inter-frame correlation decreases; for a frame separation of $\delta$, the inter-frame correlation is $\exp (-|\delta| / \gamma)$, where $\gamma$ is the temporal exponential decay factor in units of frames. Frames with large time difference, $|\delta|>d$, are considered independent. The one-step inverse (4.4) for image reconstruction then becomes

$$
\tilde{\mathbf{B}}=\tilde{\mathbf{P}} \tilde{\mathbf{J}}^{T}\left(\tilde{\mathbf{J}} \tilde{\mathbf{P}} \tilde{\mathbf{J}}^{T}+\lambda^{2} \tilde{\mathbf{V}}\right)^{-1}
$$

where $\tilde{\mathbf{V}}=\mathbf{I} \otimes \mathbf{V} . \tilde{\mathbf{V}}$ is diagonal since measurement noise is uncorrelated between frames. $\tilde{\mathbf{P}}=\boldsymbol{\Gamma} \otimes \mathbf{P}$, where $\boldsymbol{\Gamma}$ is the temporal weight matrix of an image sequence $\tilde{\mathbf{x}}$ and is defined to have the form as

$$
[\boldsymbol{\Gamma}]_{i, j}=\exp \left(-\frac{|i-j|}{\gamma}\right) \quad i, j=-d \ldots d
$$

From (4.7) and (4.8),

$$
\tilde{\mathbf{B}}=\left[\boldsymbol{\Gamma} \otimes\left(\mathbf{P} \mathbf{J}^{T}\right)\right]\left[\mathbf{\Gamma} \otimes\left(\mathbf{J P J} \mathbf{J}^{T}\right)+\lambda^{2}(\mathbf{I} \otimes \mathbf{V})\right]^{-1}
$$

Given $\tilde{\mathbf{B}}$, the one step solution for the current image $\left(\hat{\mathbf{x}}_{t}\right)$ is rewritten as

$$
\hat{\mathbf{x}}_{t}=\tilde{\mathbf{B}}_{0} \tilde{\mathbf{y}}_{t}
$$

where $\tilde{\mathbf{B}}_{0}$ is the rows $n_{M} \times d+1 \ldots n_{M} \times(d+1)$ of $\tilde{\mathbf{B}}$.

\section{Parameter selection}

The $\gamma$ is a hyperparameter of the system; it depends on the data acquisition frame rate, the speed of underlying conductivity changes and the system noise level. This section develops an approach to estimate the value of $\gamma$ from the measurement sequence. By taking covariance on both sides of (4.6), we have the estimated covariance matrix of the data as

$$
\Sigma_{\tilde{\mathbf{y}}}=\tilde{\mathbf{J}} \Sigma_{\tilde{\mathbf{x}}} \tilde{\mathbf{J}}^{t}+\Sigma_{\tilde{\mathbf{n}}}
$$


the optimal $\gamma$ is chosen so that the error between the true data covariance matrix $\Sigma_{\tilde{\mathbf{y}}}$ and the estimated one $\hat{\boldsymbol{\Sigma}}_{\tilde{\mathbf{y}}}$ is minimized as

$$
\gamma=\underset{\gamma}{\arg \min }\left\|\boldsymbol{\Sigma}_{\tilde{\mathbf{y}}}-\boldsymbol{\Sigma}_{\tilde{\mathbf{n}}}-\tilde{\mathbf{J}} \boldsymbol{\Sigma}_{\tilde{\mathbf{x}}} \tilde{\mathbf{J}}^{t}\right\|_{F}^{2}
$$

where the subscript $F$ is the Frobenius norm. Since $\boldsymbol{\Sigma}_{\tilde{\mathbf{x}}}=\mathbf{\Gamma} \otimes \boldsymbol{\Sigma}_{\mathbf{X}}$ and $\tilde{\mathbf{J}}=\mathbf{I} \otimes \mathbf{J},(4.12)$ becomes

$$
\gamma=\underset{\gamma}{\arg \min }\left\|\boldsymbol{\Sigma}_{\tilde{\mathbf{y}}}-\boldsymbol{\Sigma}_{\tilde{\mathbf{n}}}-\boldsymbol{\Gamma} \otimes\left(\mathbf{J} \boldsymbol{\Sigma}_{\mathbf{X}} \mathbf{J}^{t}\right)\right\|_{F}^{2}
$$

By taking covariance on both sides of (4.1), we have

$$
\Sigma_{\mathbf{y}}=\mathbf{J} \Sigma_{\mathbf{X}} \mathbf{J}^{t}+\Sigma_{\mathbf{n}}
$$

so that $\mathbf{J} \boldsymbol{\Sigma}_{\mathbf{X}} \mathbf{J}^{t}=\boldsymbol{\Sigma}_{\mathbf{y}}-\boldsymbol{\Sigma}_{\mathbf{n}}$; we also have $\boldsymbol{\Sigma}_{\tilde{\mathbf{n}}}=\mathbf{I} \otimes \boldsymbol{\Sigma}_{\mathbf{n}}$ and $\boldsymbol{\Sigma}_{\tilde{\mathbf{y}}}=\boldsymbol{\Gamma}_{\mathbf{y}} \otimes \boldsymbol{\Sigma}_{\mathbf{y}}$, where $\Gamma_{\mathbf{y}} \in \mathbb{R}^{(2 d+1) \times(2 d+1)}$ is the correlation matrix of $\tilde{\mathbf{y}}$. Thus the optimal $\gamma$ is calculated by

$$
\gamma=\underset{\gamma}{\arg \min }\left\|\boldsymbol{\Gamma}_{\mathbf{y}} \otimes \boldsymbol{\Sigma}_{\mathbf{y}}-\mathbf{I} \otimes \boldsymbol{\Sigma}_{\mathbf{n}}-\mathbf{\Gamma} \otimes\left(\boldsymbol{\Sigma}_{\mathbf{y}}-\boldsymbol{\Sigma}_{\mathbf{n}}\right)\right\|_{F}^{2}
$$

$\boldsymbol{\Gamma}_{\mathbf{y}}$ and $\boldsymbol{\Sigma}_{\mathbf{y}}$ can be calculated directly from the data. $\boldsymbol{\Sigma}_{\mathbf{n}}$ can be measured by calibration of EIT system. For computational efficiency, (4.15) can be simplified as

$$
\gamma=\underset{\gamma}{\arg \min }\left\|\boldsymbol{\Gamma}_{\mathbf{y}}\right\| \boldsymbol{\Sigma}_{\mathbf{y}}\left\|_{F}^{2}-\mathbf{I}\right\| \boldsymbol{\Sigma}_{\mathbf{n}}\left\|_{F}^{2}-\boldsymbol{\Gamma}\right\| \boldsymbol{\Sigma}_{\mathbf{y}}-\boldsymbol{\Sigma}_{\mathbf{n}}\left\|_{F}^{2}\right\|_{F}^{2}
$$

where $\boldsymbol{\Gamma}_{\mathbf{y}},\left\|\boldsymbol{\Sigma}_{\mathbf{y}}\right\|_{F}^{2},\left\|\boldsymbol{\Sigma}_{\mathbf{n}}\right\|_{F}^{2}$ and $\left\|\boldsymbol{\Sigma}_{\mathbf{y}}-\boldsymbol{\Sigma}_{\mathbf{n}}\right\|_{F}^{2}$ may be precalculated. Since $\boldsymbol{\Gamma}$ is relatively small $\left(\mathbb{R}^{(2 d+1) \times(2 d+1)}\right)$ this optimization is performed directly by bisection search between limits.

\subsubsection{3-D spatial prior with full model correlation}

The most common assumption for image prior models is to consider independent image elements; examples are the zeroth order Tikhonov prior (Yorkey et al., 1987), and the NOSER prior (Cheney et al., 1990). When elements are independent, the inter-element correlation is zero, meaning $\boldsymbol{\Sigma}_{x}$ is diagonal. Another common assumption is to consider that elements are locally correlated (Vauhkonen et al., 1999). In this case $\boldsymbol{\Sigma}_{x}$ has a sparse non-diagonal structure since only adjacent elements are considered correlated. We consider that these approaches have two important limitations. First, they do not reflect adequately 
the scale of the correlations in the images; image elements are correlated globally. Secondly, the spatial frequency behaviour will depend on the size of the image elements, with a larger spatial correlation being imposed for larger elements. Thus, a fine and coarse model with the same spatial prior do not really implement the same prior model, and the prior model will be spatially non-uniform for a model with localized mesh refinement.

In this section, we develop a 3-D spatial prior model which accounts for the full correlation model. It is more computationally expensive to compute than simpler prior models, but this is only performed as a precalculation, not during image reconstruction. This model is based on three factors: 1) a sensitivity weighting, where elements are weighted by the norm of the measurement sensitivity, 2) exponentially decreasing spatial correlation with inter-element distance, and 3) an additional smoothness constraint for elements in regions with poor sensitivity, such as above and below the electrode planes.

\section{Sensitivity weighting}

The sensitivity of measurements to a change in element $i$ is the norm of the $i$ th column of the Jacobian, $\left\|\mathbf{J}_{:, i}\right\|=\left[\mathbf{J}^{T} \mathbf{J}\right]_{i, i}$. As mentioned, we consider this weighting to be too strong for high sensitivity elements, and tends to push image artefacts toward the center where the sensitivity is much lower. Instead, we use a sensitivity weighting of $p=0.5$. The choice of $p$ is a heuristic compromise between the pushing noise to the boundary $(p=0)$ or to the centre $(p=1)$.

The inter-element sensitivity is based on the product of the square root of the sensitivity to each element. Thus, we define the sensitivity weighting part of the prior matrix $\mathbf{P}_{N}^{\frac{1}{2}}$ as

$$
\left[\mathbf{P}_{N}^{\frac{1}{2}}\right]_{i, i}=\left[\mathbf{J}^{T} \mathbf{J}\right]_{i, i}^{-\frac{p}{2}}
$$

Based on (4.17) the 3-D spatial prior is defined as

$$
\mathbf{P}=\mathbf{P}_{N}^{\frac{1}{2}} \mathbf{P}_{C} \mathbf{P}_{N}^{\frac{1}{2}}
$$

where $\mathbf{P}_{C}$ is the spatial correlation matrix

\section{Exponential spatial correlations}

Given two small image elements $i, j$, centred at $\mathbf{r}_{i}=\left(x_{i}, y_{i}, z_{i}\right)$ and $\mathbf{r}_{j}=\left(x_{j}, y_{j}, z_{j}\right)$, the spatial correlation is defined in terms of a spatial exponential constant $\eta$ in units of distance. Elements closer together than $\eta$ are highly correlated, and those further apart have low 
correlation. Thus

$$
\left[\mathbf{P}_{C}\right]_{i, j}=\exp \left(-\frac{\left\|\mathbf{r}_{i}-\mathbf{r}_{j}\right\|}{\eta}\right)
$$

However, for larger finite elements, (4.19) has to be modified because not all parts of each image element are at distance $\left\|\mathbf{r}_{i}-\mathbf{r}_{j}\right\|$ from each other. Instead we replace it with

$$
\left[\mathbf{P}_{C}\right]_{i, j}=\frac{1}{V_{i} V_{j}} \int_{E_{i}} \int_{E_{j}} \exp \left(-\frac{\left\|\mathbf{r}_{i}-\mathbf{r}_{j}\right\|}{\eta}\right) d \mathbf{r}_{j} d \mathbf{r}_{i}
$$

where the integrals are over the volume of finite elements $E_{i}$ and $E_{j} . V_{i}$ and $V_{j}$ are the volumes of each element, where $V_{i}=\int_{E_{i}} d \mathbf{r}_{i}$. We derive a closed form estimate for the integrals in (4.20) below.

\section{Low sensitivity constraint}

The EIT sensitivity is extremely low for regions above and below the electrode bands. This has important consequences for image reconstruction because it further increases the illconditioning of the EIT inverse. The consequence is that the algorithm may "push" image artefacts to these regions; since the measurement norm from these regions is so small, large artefacts have only a small effect on the data fit. To avoid this effect, it is possible to tightly crop the 3D FEM model near the electrode planes, but this also introduces artefacts, since it prevents the model current from flowing into these areas.

Using the exponential spatial correlations, it is possible to naturally account for low sensitivity regions by imposing a strong smoothness constraint. By dramatically increasing $\eta$ for image elements in these regions, we force the algorithm to consider elements to be highly correlated, and reduce the effective degrees of freedom (or number of fitted parameters) allocated to this region. This has the advantage that it will reduce high spatial frequency artefacts, but does not arbitrarily and unnaturally impose a penalty on reconstructed image amplitude in these regions. Thus we modify (4.20),

$$
\begin{aligned}
& {\left[\mathbf{P}_{C}\right]_{i, j}=\frac{1}{V_{i} V_{j}} \int_{E_{i}} \int_{E_{j}} \exp \left(-\frac{\left\|\mathbf{r}_{i}-\mathbf{r}_{j}\right\|}{\eta\left(\mathbf{r}_{i}, \mathbf{r}_{j}\right)}\right) d \mathbf{r}_{j} d \mathbf{r}_{i}} \\
& \text { where } \eta\left(\mathbf{r}_{i}, \mathbf{r}_{j}\right)= \begin{cases}\eta_{0} & \text { if } z_{p 1} \leq z_{i} \leq z_{p 2} \text { or } z_{p 1} \leq z_{j} \leq z_{p 2} \\
K\left(z_{i}, z_{j}\right) \eta_{0} & \text { otherwise. }\end{cases}
\end{aligned}
$$

where $\eta_{0}$ is the value used in the central regional, $z_{p 1}$ and $z_{p 2}$ are the vertical positions of the electrode planes, and $K\left(z_{i}, z_{j}\right)$ is a penalty term which may depend on the distance from the $z_{p 1}$ and $z_{p 2}$. For simplicity, we use a constant value of $K=5$ in this chapter. Note that the low sensitivity penalty is only imposed if both $z_{i}$ and $z_{j}$ are outside the electrode plane. 


\section{Integral of exponential correlations}

The integrals in (4.20) are over the coordinates of each finite element simplex. In order to develop a closed form integral we model each element instead as a sphere of the same volume and centre coordinates as the original finite element simplex. For a simplex of coordinates $\left(x_{k}, y_{k}, z_{k}\right), k=1 \ldots 4$, we have volume $V=\frac{1}{3 !} \operatorname{det} D$, where the $k$ th row of $D_{k,:}=\left[1, x_{k}, y_{k}, z_{k}\right]$. From this volume, the sphere radius is $r=\sqrt[3]{\frac{3 V}{4 \pi}}$. With this model, the integral may be approximated as a 1-D integral over the line from $\mathbf{r}_{i}$ to $\mathbf{r}_{j}$. Based on element centres, $\left(\mathbf{r}_{i}, \mathbf{r}_{j}\right)$ and radii $\left(r_{i}, r_{j}\right)$, we calculate the $(i, j)$ element of the 3 -D spatial prior $\mathbf{P}_{C}$ as follows:

$$
\left[\mathbf{P}_{C}\right]_{i, j}=\frac{1}{V_{i} V_{j}} \int_{-r_{i}}^{r_{i}} \int_{-r_{j}}^{r_{j}} \exp \left(-\frac{\left|\Delta_{i j}+x+y\right|}{\eta}\right) d y d x
$$

where $V_{i}=2 r_{i}, V_{j}=2 r_{j}$ and $\Delta_{i j}=\left\|\mathbf{r}_{i}-\mathbf{r}_{j}\right\|$.

Because of the absolute value, the inner integral has two cases:

$$
\int_{-r_{j}}^{r_{j}} e^{-\frac{1}{\eta}\left|\Delta_{i j}+x+y\right|} d y=\eta e^{\frac{\Delta_{i j}+x}{\eta}}\left[e^{\frac{y}{\eta}}\right]_{-r_{j}}^{y^{\prime}}-\eta e^{-\frac{\Delta_{i j}+x}{\eta}}\left[e^{-\frac{y}{\eta}}\right]_{y^{\prime}}^{r_{j}}
$$

where $y^{\prime}=-\left(\Delta_{i j}+x\right)$ if $-r_{j} \leq \Delta_{i j}+x \leq r_{j}$ and is otherwise limited to the range $y^{\prime}= \pm r_{j}$. Based on these cases, the outer integral is split into three portions $-r_{i} \leq x \leq x^{\prime}$, $x^{\prime}<x<x^{\prime \prime}$, and $x^{\prime \prime}<x<r_{i}$, where $x^{\prime}=\min \left(r_{i},-\Delta_{i j}-r_{j}\right)$ and $x^{\prime \prime}=\min \left(r_{i},-\Delta_{i j}+r_{j}\right)$. Thus

$$
\begin{aligned}
{\left[\mathbf{P}_{C}\right]_{i, j} } & =\frac{\eta}{4 r_{i} r_{j}}\left(\int_{-r_{i}}^{x^{\prime}} e^{\frac{\Delta_{i j}+x}{\eta}}\left[e^{\frac{y}{\eta}}\right]_{-r_{j}}^{r_{j}} d x+\int_{-x^{\prime \prime}}^{r_{i}} e^{-\frac{\Delta_{i j}+x}{\eta}}\left[e^{\frac{y}{\eta}}\right]_{-r_{j}}^{r_{j}} d x\right. \\
& \left.+\int_{-x^{\prime}}^{x^{\prime \prime}} e^{\frac{\Delta_{i j}+x}{\eta}}\left[e^{\frac{y}{\eta}}\right]_{-r_{j}}^{-\left(\Delta_{i, j}+x\right)}+e^{-\frac{\Delta_{i j}+x}{\eta}}\left[e^{\frac{y}{\eta}}\right]_{-r_{j}\left(\Delta_{i, j}+x\right)} d x\right) \\
& =\frac{\eta^{2}}{4 r_{i} r_{j}}\left(e^{-\frac{\Delta_{i j}}{\eta}}\left[e^{\frac{x}{\eta}}\right]_{-r_{i}-x^{\prime \prime}}^{+r_{i}+x^{\prime}}\left[e^{\frac{y}{\eta}}\right]_{-r_{j}}^{+r_{j}}+\left[\frac{2}{\eta} x-e^{-\frac{\Delta_{i, j}+r_{j}-x}{\eta}}+e^{-\frac{\Delta_{i, j}-r_{j}+x}{\eta}}\right]_{x^{\prime}}^{x^{\prime \prime}}\right)
\end{aligned}
$$

\subsubsection{Methods: Simulations}

Numerical simulations were designed to model the movement of blood through the mitral valve during systolic ejection. We use the following average values for healthy humans: Mitral valve area is $7.1 \pm 1.3 \mathrm{~cm}^{2}$ (Ormiston et al., 1981) and the rate of change of left ventricular volume is $473 \mathrm{ml} / \mathrm{s}$ (Hammermeister et al., 1974). These values correspond to a 
mitral radius of $1.5 \mathrm{~cm}$; we thus simulate the ventricular ejection blood flow as conductive sphere of $1.5 \mathrm{~cm}$ radius moving at $97.8 \mathrm{~cm} / \mathrm{s}$.

Numerical simulations were conducted using a 3-D cylindrical FEM model with 77999 elements using the Netgen 4.4 software. Illustrated as Fig. (4.1), the model height is $30 \mathrm{~cm}$ $(z: 0 \sim 30)$; two electrode rings ( 8 electrodes each) were attached at heights $z=10,20 \mathrm{~cm}$ using adjacent stimulation and measurement pattern. The systolic ejection is upwards and lateral which can be roughly simulated as a helical pattern movement in blood vessel. Illustrated as Fig. (4.2), inside the model, a conductive sphere with radius $1.5 \mathrm{~cm}$ rotates and rises in a the helical pattern at a uniform speed from $z=7.5 \mathrm{~cm}$ to $z=22.5 \mathrm{~cm}$, moving clockwise (bird's eye view) along a trajectory with radius $10 \mathrm{~cm}$. The background material was set to a homogeneous conductivity, $\sigma_{0}=1.0 \mathrm{~S} / \mathrm{m}$, and the spherical target to be more conductive: $\sigma=1.2 \mathrm{~S} / \mathrm{m}$. The projection of the movement trajectory on the $x-y$ plane is a full circle, and corresponds to a movement of $64.5 \mathrm{~cm}$. Thus, to model systolic ejection, one cycle of conductive sphere movement is set to be 0.66 seconds. In model an EIT system with a frame rate $\approx 40$ frames $/ \mathrm{s}$, and thus simulate a movement rate of the conductive sphere of 26 frames/cycle.

The noise performance of the algorithms was tested by adding pseudo random, zero mean Gaussian noise to each image reconstruction (with the same random seed). Images were reconstructed on a 3-D model generated by using the EIDORS software (Adler and Lionheart, 2006) with 10 vertical slices and 256 elements on each slice; this inverse model differs from the simulation model to avoid the inverse crime(D. Colton, 1998).

\subsection{Results}

Reconstructed targets were calculated using EIDORS to evaluate four algorithms with different regularization priors: 1) GN solver, with no spatial or temporal prior model; 2) temporal solver, with a temporal but no spatial prior model; 3) 3-D prior solver, with a spatial but no temporal prior model; and 4) 4-D prior solver, with both a spatial and temporal prior model. The measurement to be reconstructed was chosen for the conductive sphere in a left anterior position, and slightly closer to the bottom than the top electrode plane. Fig 4.2 shows the conductive sphere location $(x, y, z)=(3.1,-9.5,14.25)$ and that of the position of the sphere during the $d=3$ frames before and after the centre measurement used in the image reconstruction. The reconstructions were plotted as nine vertical slices chosen from $z=9 \mathrm{~cm}$ to $z=21 \mathrm{~cm}$. Figures 4.3 and 4.4 show images with Noise to Signal Ratios (NSR) of 0 and 2 , respectively. NSR was defined as $\sigma_{\mathbf{n}} / \overline{\mathbf{y}}$, where $\overline{\mathbf{y}}$ is the mean 


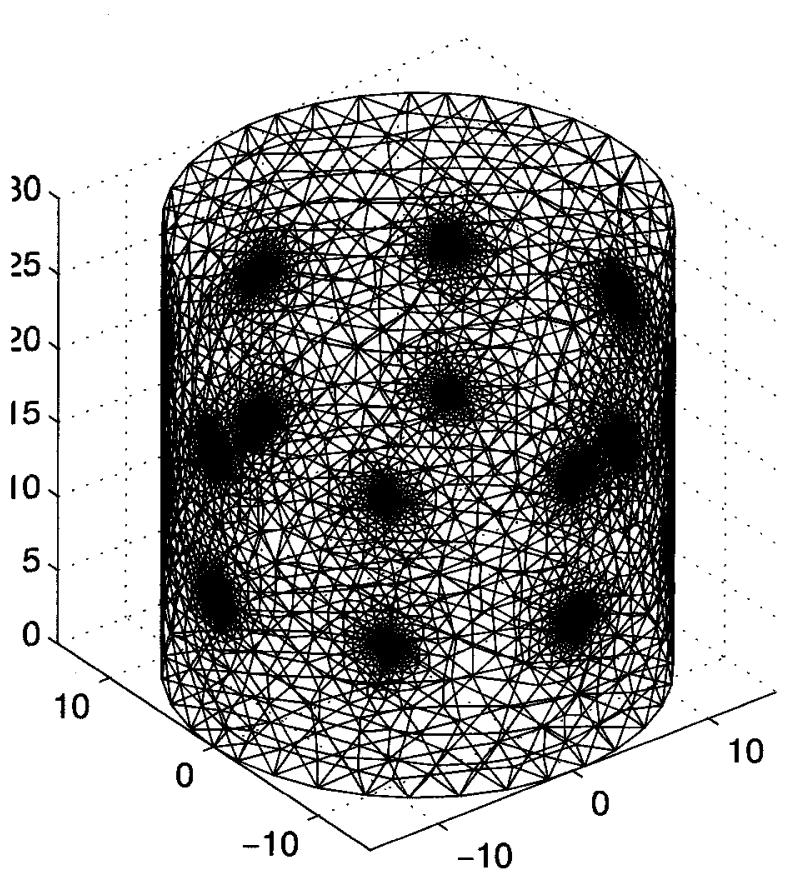

(a)

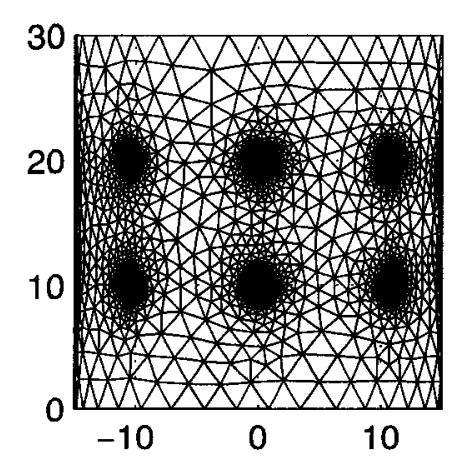

(b)

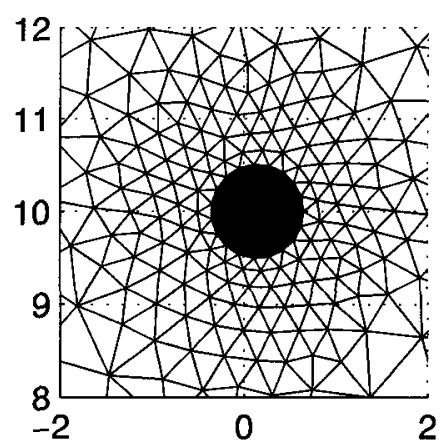

(c)

Figure 4.1: The forward model: 3-D cylindrical finite element model generated from netgen. The tank of height $30 \mathrm{~cm}$ and diameter $30 \mathrm{~cm}$ is meshed with 77999 tetrahedral elements. Sixteen circular electrodes (diameter $1.0 \mathrm{~cm}$ ) are placed in two planes (8 electrodes each) located at $z=10 \mathrm{~cm}$ and $20 \mathrm{~cm}$, respectively. Mesh refinement is applied around electrodes. (a) 3-D forward model; (b) side view, (c) one of electrodes with mesh refined around it.

value of the difference signal. The optimal value of $\gamma$ was calculated based on Section 4.3.2 as 0.97 and 0.89 frames, for $\mathrm{NSR}=0$ and $\mathrm{NSR}=2$, respectively. Different random seeds were used without evidently different results observed. The value of $\eta$ was chosen to be $3 \mathrm{~cm}$ or 0.1 of the medium diameter. This value will tend to penalize spatial frequency content in images that is less than $10 \%$ of the diameter, or $1 \%$ of the area. This value (1\%) corresponds approximately the number of independent measurements available from this EIT system (104).

In order to allow comparison across algorithms, we select identical hyperparameter for all algorithms tested. When $\lambda$ is low, there is no significant difference between the GN method and temporal/3-D/4-D priors methods. We empirically choose a relatively large 


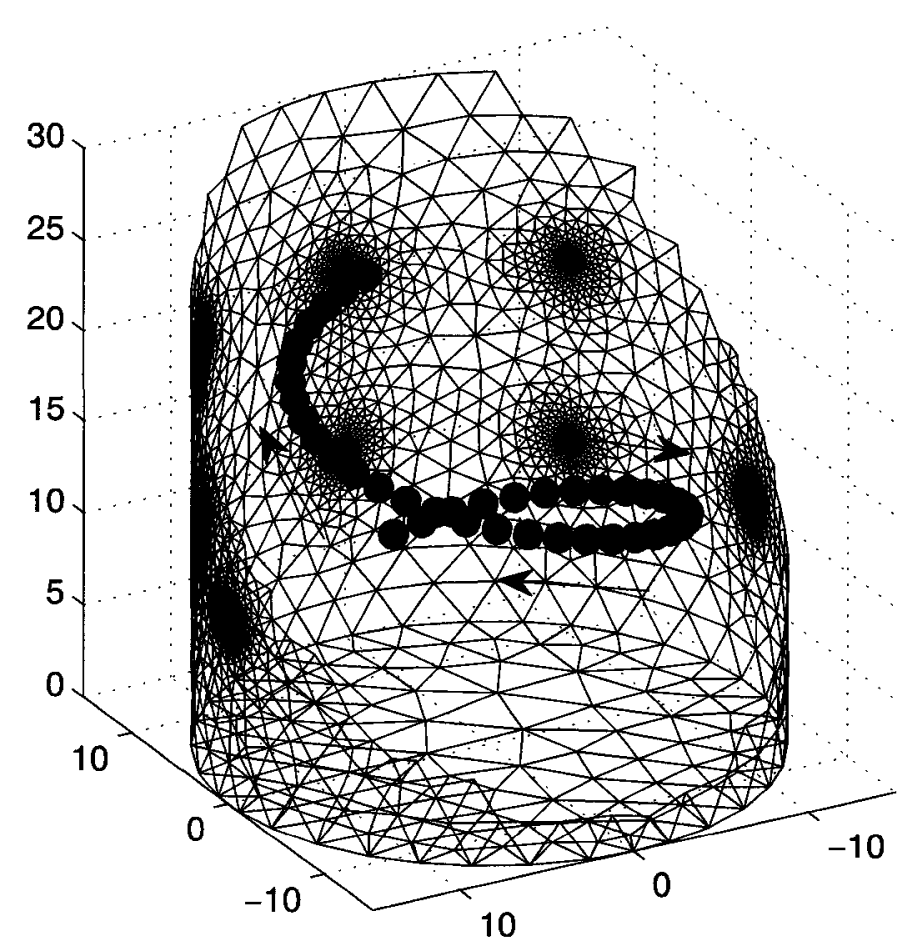

Figure 4.2: The trajectory of a spherical conductive target of radius $1.5 \mathrm{~cm}$, representing the bolus left ventricular ejection. To clarify the target movement, the tank model is cropped by the plane defined by $y+15<z$. The target $((x, y, z)=(3.1,-9.5,14.25))$ is black in the position at which the frame is taken for image reconstruction. Targets in dark blue are positions on from which data frames are measured and used in the calculation by the temporal solver ( $d=3$ as illustrated).

value of $\lambda=0.5$ to illustrate the effect in higher noise conditions. Figures 4.3 and 4.4 show reconstructed images for each algorithm with no noise and with a fairly large $(\mathrm{NSR}=2)$ level of noise. All images show reconstructions in arbitrary units and are scaled equally.

In order to evaluate the quality of reconstructed images, the following figures of merit are used: 1) target resolution: the point spread function (PSF) of the target is small in the horizontal plane, and the off plane ghosts (which illustrated as virtual targets in the planes other than the target plane $z=14.25$ ) are small; 2) reconstruction position error: the planar position of the reconstructed target is correct. The planar position of the reconstructed target is calculated from the slice at $z=14.25$ using the position definition described in (Adler and Guardo, 1996a): the centre of gravity is computed on a zone 

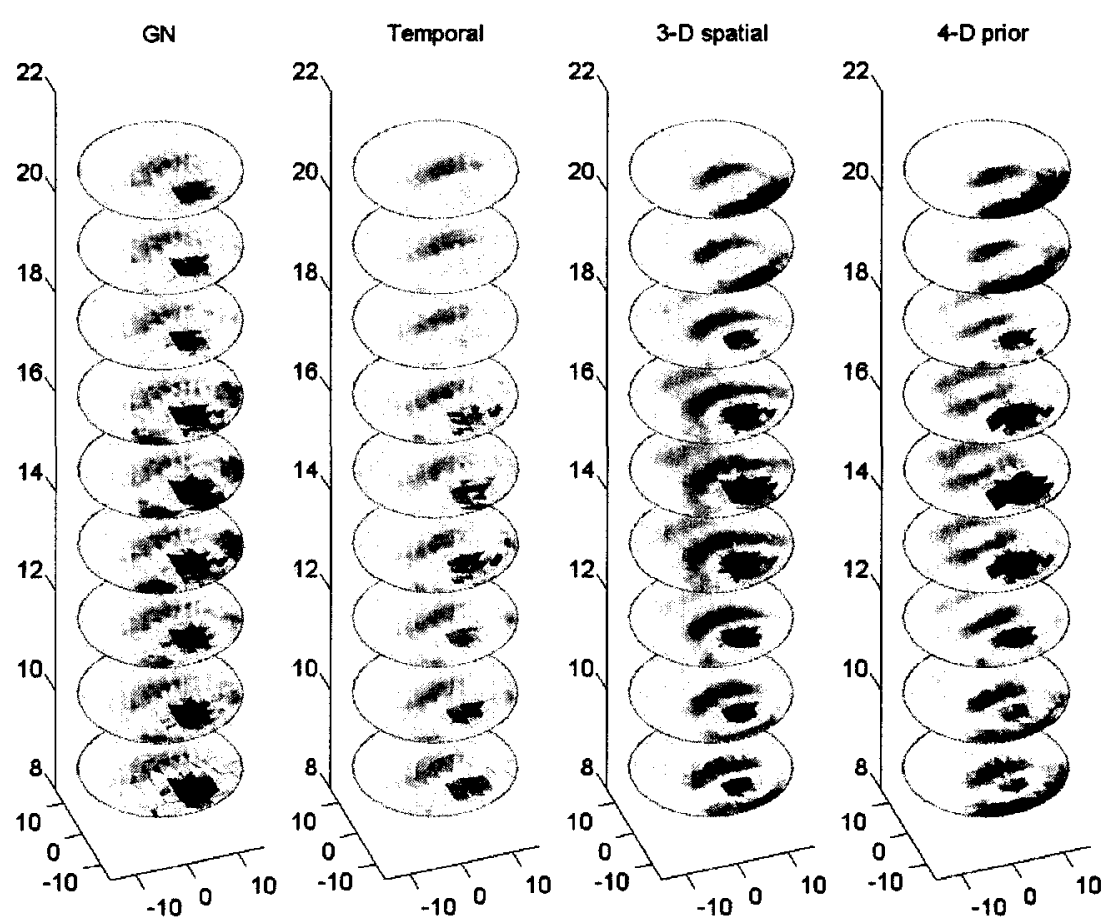

Figure 4.3: Comparison of algorithms with no noise. From right to left, algorithms are: conventional GN solver, temporal solver, 3-D prior solver, and 4-D prior solver. The image to be reconstructed was at $t=0.3 \mathrm{sec}$. Electrode planes were at $z=10$ and $20 \mathrm{~cm}$. Parameters are $\mathrm{NSR}=0, \lambda=0.5, \eta=0.1, \gamma=32.8$.

defined by half magnitude of image; and 3) noise performance: the reconstructed images should be robust to measurement noise. Based on these criteria and Figs. 4.3, 4.4 and Table 4.1, we observe that: 1) target resolution: 4-D prior solver is ranked as the highest resolution; 2) reconstruction position error: The position error (distance to the optimal position $(3.1,-9.5))$ is listed as Table 4.1. 4-D prior solver achieves best planar position accuracy for both the noisy and noise-free situations; 3) noise performance: temporal prior solver has the best noise robustness. However, 3-D prior solver introduces more artefacts when noise is added.

Figure (4.5) shows the relationship of the hyperparameter $\gamma$ to the noise and speed of conductivity change. $\gamma$ is a measure of the exponential rate of loss of coherence between images elements across frames. The figure shows, as expected, that $\gamma$ decreases as the conductivity change rate increases (or, equivalently, as the data frame rate decreases) or as the noise level increases. At very low system speed and/or very high $N S R, \gamma$ reaches 

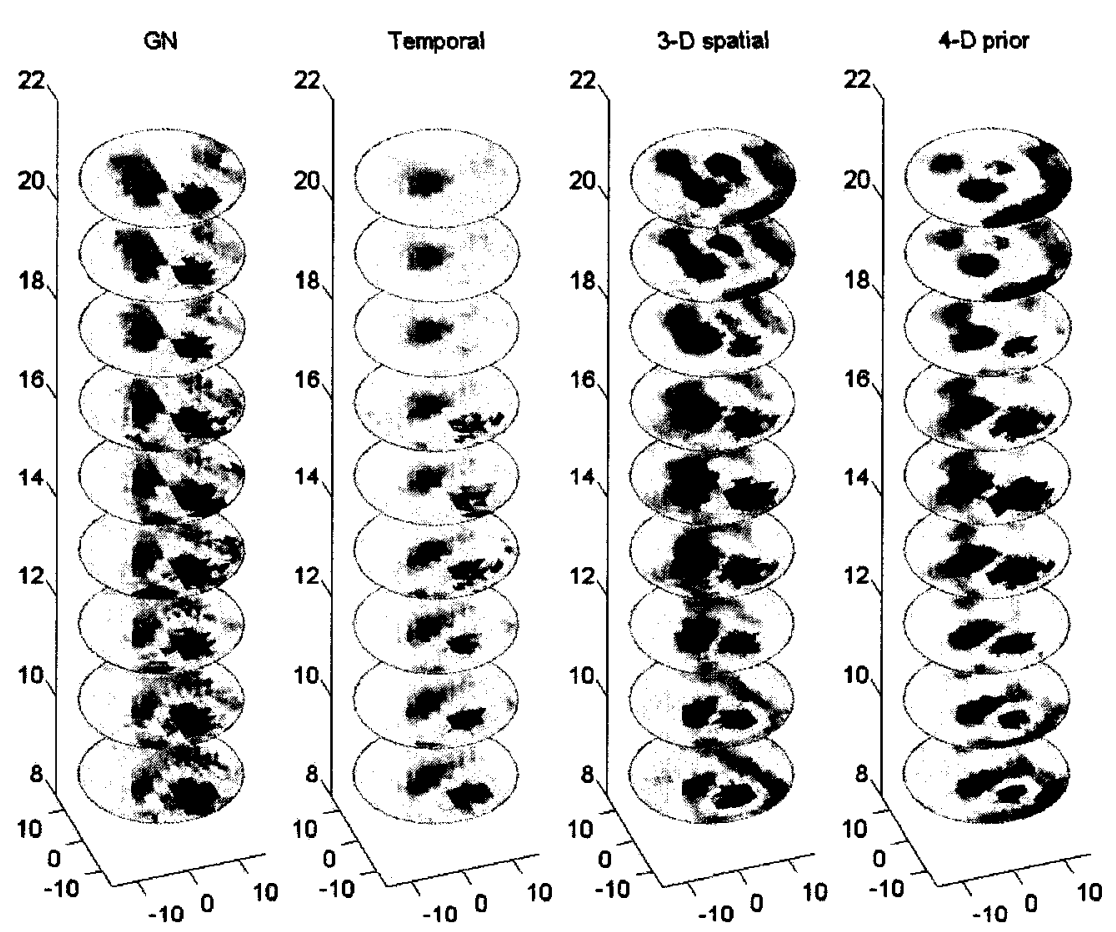

Figure 4.4: Comparison of algorithms with noise. From right to left, algorithms are: conventional GN solver, temporal solver, 3-D prior solver, and 4-D prior solver. The image to be reconstructed was at $t=0.3 \mathrm{sec}$. Electrode planes were at $z=10$ and $20 \mathrm{~cm}$. Algorithm parameters are NSR=2, $\lambda=0.5, \eta=0.1, \gamma=8.6$.

zero, indicating that adjacent frames are independent, and the temporal prior provides no advantage.

\subsection{Discussion}

Traditionally, EIT reconstruction algorithms assume each data frame to be independent. However, since EIT is able to make measurements at high frame rates, we know a priori that image frames are correlated. Intuitively, it makes sense that a sophisticated image reconstruction algorithm should be able to take advantage of known correlations in the input data to benefit the reconstructed images.

Kalman filter techniques in EIT (such as Vauhkonen et al. 1998a) provide a temporal image reconstruction based on iterative tracking. The estimated image is calculated from the current data and the previous image estimate. This differs from our proposed 
Table 4.1: The list of planar positions of reconstructed target at the level $z=14.25$, and the distances to the optimal position $(3.1,-9.5)$.

\begin{tabular}{|c|c|c|c|c|}
\hline \multirow{2}{*}{ solvers } & \multicolumn{2}{|c|}{$N S R=0$} & \multicolumn{2}{c|}{$N S R=2$} \\
\cline { 2 - 5 } & $(\mathrm{x}, \mathrm{y})(\mathrm{cm})$ & distance $(\mathrm{cm})$ & $(\mathrm{x}, \mathrm{y})(\mathrm{cm})$ & distance $(\mathrm{cm})$ \\
\hline \hline Conventional GN & $(4.15,-9.93)$ & 1.14 & $(3.93,-9.83)$ & 0.89 \\
\hline Temporal prior solver & $(3.93,-9.97)$ & 0.95 & $(3.84,-9.98)$ & 0.88 \\
\hline 3-D prior solver & $(3.91,-9.51)$ & 0.81 & $(3.95,-9.05)$ & 0.96 \\
\hline 4-D prior solver & $(3.76-9.04)$ & 0.80 & $(3.63,-8.84)$ & 0.85 \\
\hline
\end{tabular}

method which calculates a single step solution using a "frame window" with $d$ frames before and after the current frame. It improves over Kalman filter based algorithms by allowing an explicit control over the regularization prior and the weighting of measured data. The one-step temporal reconstruction method was applied to experimental data from the Magnetic Inductance Tomography (MIT) which is a similar inverse problem case to EIT (Soleimani et al., 2008). The improved reconstruction performance was also demonstrated compared to an absolute reconstruction method.

In this chapter, we carefully consider the a priori spatial and temporal correlations in EIT images. For spatial correlations, a novel exponential model is presented based on: 1) sensitivity weighting, 2) an exponentially model of inter-element correlations, and 3) a smoothness constraint for poor sensitivity regions. In order to avoid dependence on mesh element density, a closed form approximation to the integral is developed. The spatial prior model developed in this chapter models the spatial variation in conductivity as a random walk process. It has the advantage over simpler prior models (such as the discrete Laplacian and NOSER priors) of accounting for the spatial interactions in a way that is independent of element size. However, linear prior models cannot account for sharp edges and piece-wise constant conductivity regions; it is possible to use total variation priors for this case (Lionheart et al., 2005), at the expense of significantly longer iterative solutions. For the temporal correlations, an exponential model is presented of inter-frame correlations (based on an exponential constraints $\gamma$ ), and an automatic approach to determine $\gamma$ from the measurements is developed. The choice of temporal window $d$ depends on several considerations; large $d$ may decrease artefacts and reconstruction noise; however, it increases the size of the matrix inverse, and increases the condition number of the matrix to be inverted. In order to implement a temporal solver in an EIT system for real-time imaging, a delay must be introduced between the measurements and reconstruction to allow acqui- 


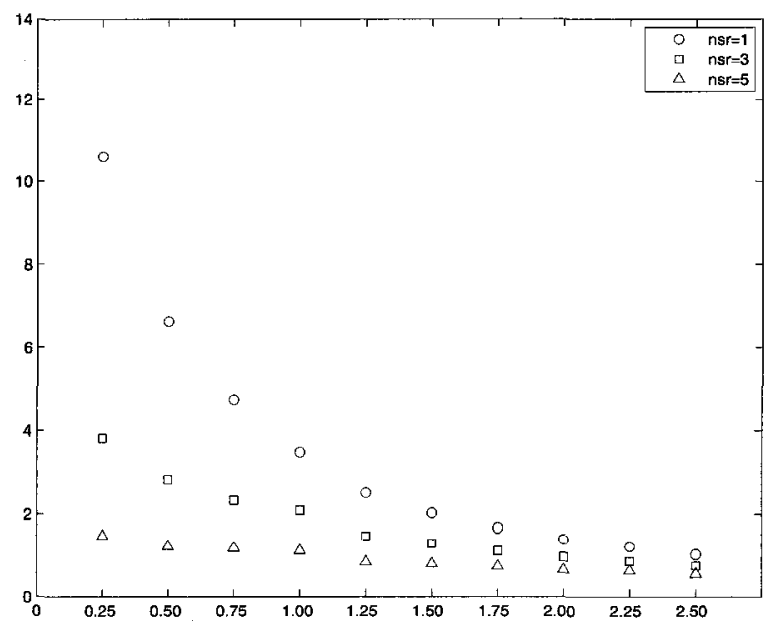

Figure 4.5: The inter-frame correlation $\gamma$ as a function of target speed and noise level. vertical axis temporal exponential decay parameter $\gamma$ (units frames) calculated from measurements. horizontal axis target frame period relative to the frame rate of $40 \mathrm{frame} / \mathrm{s}$. Image reconstruction used $d=3, \lambda=0.5$.

sition of $d$ "future frames". This corresponds to the linear phase filters used in digital signal processing applications. For fast EIT systems, with frame rates above 15 frames/sec, a choice of $d=3$ introduces a delay of $200 \mathrm{~ms}$. One core assumption of linearized time difference imaging is that conductivity variation is small. This allows the solution to assume that $\mathbf{J}$ does not vary during the $2 d+1$ frames considered in the reconstruction formulation. In the (relatively unlikely) case that $\mathbf{J}$ could not be assumed constant, the choice of $d$ would need to be limited by the rate of change of background conductivity.

Comparisons of reconstructed images with these algorithms show that increasing consideration of prior correlations does improve the reconstructed image quality. In figures. 4.3, 4.4, we compare four approaches: traditional one-step GN, GN with temporal prior, GN with 3-D spatial prior and GN with 4-D prior. These results indicate that the temporal prior provides better image SNR and resolution compared with the tradition GN method. The 3-D spatial prior shows still better resolution; however, the 3-D spatial prior is more sensitive to noise than temporal prior. The 4 -D prior shows a combined advantage with less noise sensitivity. Some interesting effects are that the GN and temporal reconstructions show more disjoint image elements, which would appear to be due to a lack of smoothness constraint. Another effect is a circumferential elongation of the reconstructed object in the direction of movement in the temporal and $4-\mathrm{D}$ reconstructions. This is a consequence of 
the temporal model accounting for additional data from times before and after the current instant.

In this chapter, the ventricular ejection blood flow is simulated as a conductive sphere which moves laterally and upward in a helical pattern. This is a simplification which matches the velocity, direction and ejection volume during systolic ejection; however, the physiological blood transport is much more complicated, which we do not simulate.

In summary, this chapter proposes a four dimensional regularization for EIT reconstruction algorithms. We demonstrate that it is advantageous to take into account any spatial and temporal correlations which exist in the underlying images. We recommend the temporal method for cases in which the data noise is high and the underlying conductivity changes are rapid with respect to the frame rate. If the frame rate is much larger than the conductivity changes, then time averaging of measurements will provide the same effect with a simpler algorithm. Similarly, if data noise is very low, it does not help to consider measurements from data frames at different times. However, we propose that the consideration of temporal and spatial correlations will generally be advantageous for EIT image reconstruction, and, most likely, for other imaging modalities as well. 


\section{Chapter 5}

\section{Reconstruction of Conductivity Changes and Electrode Movements Based on EIT Temporal Sequences}

\subsection{Summary}

This chapter is based on the paper - Reconstruction of Conductivity Changes and Electrode Movements Based on EIT Temporal Sequences, Physiological Measurement, 29:S77-S88, 2008, by Tao Dai, Camille Gómez-Laberge and Andy Adler

Electrical impedance tomography (EIT) reconstructs a conductivity change image within a body from electrical measurements on body surface; while it has relatively low spatial resolution, it has a high temporal resolution. One key difficulty with EIT measurements is due to the movement and position uncertainty of the electrodes, especially due to breathing and posture change. In this chapter, we develop an approach to reconstruct both the conductivity change image and the electrode movements from the temporal sequence of EIT measurements. Since both the conductivity change and electrode movement are slow with respect to the data frame rate, there are significant temporal correlations which we formulate as priors for the regularized image reconstruction model. Image reconstruction is posed in terms of a regularization matrix and Jacobian matrix which are augmented for the conductivity change and electrode movement, and then further augmented to concatenate the $d$ previous and future frames. Results are shown for simulation, phantom and human data, and show that the proposed algorithm yields improved resolution and noise 
performance in comparison to a conventional one-step reconstruction method.

\subsection{Introduction}

Electrical Impedance Tomography (EIT) calculates an estimate of the conductivity distribution within a body based on current stimulations and voltage measurements on the body surface. EIT imaging has low spatial resolution, however, EIT can have excellent temporal resolution. Some recent systems have frame rates up to $1000 \mathrm{fps}$ (Wilkinson et al., 2005). Such high temporal resolution makes EIT a promising technology to monitor fast physiological events which affect the conductivity distribution.

For cardiac activities, EIT can locally determine impedance variations in ventricular or atrial regions during the cardiac cycle (Eyuboglu et al., 1989) and calculate cardiac parameters such as stroke volume (Vonk-Noordegraaf et al., 2000). For pulmonary function monitoring, EIT has been demonstrated as an effective tool due to the conductivity variation of lungs is highly related to the air ventilation (Dijkstra et al., 1993), blood infusion (McArdle et al., 1988), and intrathoracic fluid volumes (Campbell et al., 1994). EIT is helpful for imaging rapid conductivity changes due to brain neuronal activity, which occur within a timescale of milliseconds, such as acute blood infusion/exfusion from visually evoked responses (Holder, 1987; Tidswell et al., 2001).

Due to the diffusive propagation of electrical current in the human body, EIT is a soft field tomography modality. Compared with the number of pixels/voxels to be reconstructed, the amount of electrodes that can be applied on body surface is relatively small. Thus, the reconstruction of an unknown internal conductivity distribution from boundary data is severely ill-conditioned (Lionheart et al., 2005). In order to calculate a "reasonable" image, regularization techniques are required. Such regularized image reconstructions can be statistically formulated in terms of a priori information about image element values and the correlations among them. In many EIT algorithms, the zeroth order Tikhonov (Vauhkonen et al., 1998b), discrete Laplacian filter (Polydorides and Lionheart, 2002) and the NOSER priors (Cheney et al., 1990; Graham and Adler, 2007) are commonly used. Those regularization priors treat all images independently from each other. However, it is clear that images within a certain temporal distance are not independent but do contain useful temporal correlations, especially for high speed EIT. This type of temporal correlation was exploited by Adler et al. (2007) to improve EIT image noise performance and resolution.

The position uncertainty of electrodes is a principle source of artefacts and reconstruc- 
tion errors. Difference EIT is less sensitive to electrode position uncertainty assuming the electrodes do not move during measurement (Barber and Brown, 1988). However, this assumption is not valid in medical applications, such as cardiopulmonary imaging, in which the chest moves during breathing and/or posture change. Harris et al. (1988), Adler et al. (1996b) and Zhang and Patterson (2005) showed the electrode movement introduced by thoracic variation had a significant effect on EIT imaging.

In this chapter, we address two issues in EIT, the electrode position uncertainty and data noise, by taking advantage of the high frame rate of modern EIT systems. Previously, we developed an approach to reconstruct conductivity change and electrode movement from a single frame of EIT data using an augmented Jacobian and prior matrix (Soleimani et al., 2006a) . One limitation of this approach is that reconstructed electrode movements can often be noisy. This chapter develops an algorithm to improve reconstructions of both electrode movements and conductivity changes by considering correlations between reconstructions in successive data frames. We take advantage of the fact that with fast EIT systems, the boundary shape and internal conductivities change relatively slowly, and may be formulated as a priori constraints in the image reconstruction model. This chapter proposes to reconstruct EIT images via an inverse problem with a regularization prior that accounts for both spatial and temporal correlations among image elements and electrode movements. This algorithm is verified by numerical simulation, saline phantom data and in vivo human measurement.

\subsection{Methods}

An EIT system has $n_{E}$ electrodes applied on a body surface in a plane using the adjacent current stimulation and voltage measurement. Also $n_{E}$ current stimulation patterns are sequentially applied and each of them takes $n_{E}-3$ differential measurements. Each data frame measures a vector, $\mathbf{v} \in \mathbb{R}^{n_{M}}$, of $n_{M}=n_{E}\left(n_{E}-3\right)$ data points (some of which are redundant if the medium is not changing). Difference EIT calculates difference data $\mathbf{y}$, $\mathbf{y}_{i}=\mathbf{v}_{i}-\mathbf{v}_{0}$, where $i$ is the time index. To improve its precision, the reference signal $\mathbf{v}_{0}$ is typically the average over many data frames that are acquired when the measured object may be assumed to be stable. Therefore, $\mathbf{v}_{0}$ is assumed noise free.

The body investigated is modelled using a finite element model (FEM) that discretizes the body conductivity into $n_{N}$ piecewise smooth elements, represented by a vector $\sigma \in \mathbb{R}^{n_{N}}$. Difference EIT calculates a vector of conductivity changes, $\mathbf{x}_{i}=\sigma_{i}-\sigma_{0}$ between the present conductivity distribution, $\sigma_{i}$, and that at the reference measurement $\sigma_{0}$. For small 
variations around the reference conductivity $\sigma_{0}$, the relationship between $\mathbf{x}$ and $\mathbf{y}$ can be linearized so that the difference EIT forward model is:

$$
\mathbf{y}=\mathbf{J x}+\mathbf{n}
$$

where $\mathbf{J} \in \mathbb{R}^{n_{M} \times n_{N}}$ is the Jacobian or sensitivity matrix and $\mathbf{n} \in \mathbb{R}^{n_{M}}$ is the vector of measurement noise, which is assumed to be uncorrelated white Gaussian. The Jaco-

bian is calculated from the FEM as $\mathbf{J}_{i j}=\left.\frac{\partial \mathbf{y}_{i}}{\partial \mathbf{x}_{j}}\right|_{\sigma_{0}}$, and depends on the FEM, current injection patterns, the reference conductivity and the electrode models. This system is under-determined since $n_{N}>n_{M}$. This problem is commonly solved using regularization techniques in order to calculate an estimated conductivity change $\hat{\mathbf{x}}$, which is both faithful to the measurements $\mathbf{y}$ and to a priori constraints on a "reasonable" image.

Under the condition that the system is relatively stable ( $\mathbf{J}$ is constant), a sequence of difference data $\mathbf{y}_{i}$ are obtained. As long as the conductivity of the body measured doesn't change too rapidly, it is reasonable to expect that a certain number $d$ of adjacent frames in the past and from the future provide useful hints about the current image. Labelling the current instant as $t$, we then seek to estimate $\hat{\mathbf{x}}_{t}$ from the data $\left[\mathbf{y}_{t-d} ; \ldots ; \mathbf{y}_{t-1} ; \mathbf{y}_{t} ; \mathbf{y}_{t+1} ; \ldots ; \mathbf{y}_{t+d}\right]$.

In the subsequent sections we consider the following methods: 5.3.1) Traditional GN inverse, using $\mathbf{y}_{t}$ only, to reconstruct conductivity change. 5.3.2) GN inverse calculating both conductivity change and electrode movement, using $\mathbf{y}_{t}$ only, to reconstruct both conductivity change and electrode movement. 5.3.3) Temporal $G N$ inverse, using $\mathbf{y}_{t-d} \ldots \mathbf{y}_{t+d}$, to reconstruct conductivity change only based on a temporal prior model. 5.3.4) Temporal inverse on both conductivity change and electrode movement, using $\mathbf{y}_{t-d} \ldots \mathbf{y}_{t+d}$, to reconstruct both conductivity change and electrode movement based on a temporal prior model.

\subsubsection{One-step linear Gauss-Newton (GN) solver}

The regularized image reconstruction based on the one-step linearized GN method was first introduced into EIT by Yorkey et al. (1987) and has been widely used (e.g., Cheney et al. (1990); Adler and Guardo (1996a)). It addresses the inverse solution as a linear reconstruction matrix and allows use of advanced regularization methods to solve the inverse problem. By using a precalculated reconstruction matrix, it can realize rapid, real-time imaging. The GN inverse problem estimates a solution $\hat{\mathbf{x}}$ by minimizing

$$
\|\mathbf{y}-\mathbf{J} \hat{\mathbf{x}}\|_{\Sigma_{n}^{-1}}^{2}+\left\|\mathbf{x}-\mathbf{x}^{\circ}\right\|_{\Sigma_{x}^{-1}}^{2}
$$


where $\boldsymbol{\Sigma}_{n} \in \mathbb{R}^{n_{M} \times n_{M}}$ is the covariance matrix of the measurement noise $\mathbf{n}$. Since noise channels are independent, $\boldsymbol{\Sigma}_{n}$ is a diagonal matrix with $\left[\boldsymbol{\Sigma}_{n}\right]_{i, i}=\sigma_{i}^{2}$, where $\sigma_{i}^{2}$ is the noise variance at channel $i$. Here $\boldsymbol{\Sigma}_{x} \in \mathbb{R}^{n_{N} \times n_{N}}$ is the covariance matrix of the desired image and $\mathbf{x}^{\circ}$ represents the expected value of image, which is zero for difference EIT.

Instead of calculating $\boldsymbol{\Sigma}_{n}$ and $\boldsymbol{\Sigma}_{x}$, we heuristically model them from a priori considerations by introducing $\mathbf{W}=\sigma_{n}^{2} \boldsymbol{\Sigma}_{n}^{-1}$ and $\mathbf{R}=\sigma_{x}^{2} \boldsymbol{\Sigma}_{x}^{-1}$. Here $\sigma_{n}$ is the average measurement noise amplitude and $\sigma_{x}$ is the a priori amplitude of conductivity changes. The measurement accuracy is modelled by $\mathbf{W}$. For uncorrelated noise, each diagonal element of $\mathbf{W}$ is proportional to the corresponding channel signal-to-noise-ratio (SNR). For difference EIT with identical channels, $\mathbf{W}$ is an identity matrix; or else, it may be measured during the system calibration test (the identity matrix is used in this chapter for simplicity). The regularization matrix $\mathbf{R}$ may be understood to statistically model the amplitudes and interactions of image elements. Simply, $\mathbf{R}$ may consider all elements equally alike and independent (or only locally dependent). More sophisticated models (e.g., Dai et al. (2007)) may consider smooth distributions more likely than rapidly changing ones and using temporal/spatial correlations.

From (5.2), a linearized one-step inverse solution is obtained as

$$
\hat{\mathbf{x}}=\left(\mathbf{J}^{T} \mathbf{W} \mathbf{J}+\lambda^{2} \mathbf{R}\right)^{-1} \mathbf{J}^{T} \mathbf{W} \mathbf{y}=\mathbf{B y}
$$

where $\lambda=\sigma_{n} / \sigma_{x}$ is the regularization parameter, or hyperparameter, which controls the trade-off between resolution and noise attenuation in the reconstructed image. Here $\mathbf{B}=$ $\left(\mathbf{J}^{T} \mathbf{W} \mathbf{J}+\lambda^{2} \mathbf{R}\right)^{-1} \mathbf{J}^{T} \mathbf{W}$ is the linear, one-step reconstruction matrix.

Assuming that image elements are independent and have an identical expected magnitude, $\mathbf{R}$ becomes an identity matrix $\mathbf{I}$ and (5.3) uses zeroth order Tikhonov regularization. For EIT, such solutions tend to push reconstructed noise toward the boundary, since the measured data are much more sensitive to boundary elements than deep elements. In order to compensate the sensitivity discrepancy, $\mathbf{R}$ may be scaled by the sensitivity of elements, so that $\mathbf{R}$ is a diagonal matrix with elements $[\mathbf{R}]_{i, i}=\left[\mathbf{J}^{T} \mathbf{J}\right]_{i, i}^{p}$. This is the NOSER prior (Cheney et al., 1990) with an exponent $p$. In this chapter, the NOSER prior is used in all tested algorithms. The exponent is chosen as $p=0.5$ heuristically, as a compromise between the pushing noise to the boundary $(p=0)$ or to the centre $(p=1)$.

The term inverted in (5.3) is of size $n_{N} \times n_{N}$. The matrix $\mathbf{B}$ can be rewritten using the data form (Adler et al., 2007):

$$
\mathbf{B}=\mathbf{P J}^{T}\left(\mathbf{J P J} \mathbf{J}^{T}+\lambda^{2} \mathbf{V}\right)^{-1}
$$


where $\mathbf{P}=\mathbf{R}^{-1}=\boldsymbol{\Sigma}_{x} / \sigma_{x}^{2}$ and $\mathbf{V}=\mathbf{W}^{-1}=\boldsymbol{\Sigma}_{n} / \sigma_{n}^{2}$. In (5.4), the size of the inverted matrix is reduced to $n_{M} \times n_{M}$. This is important for large scale models, such as 3D EIT models and the temporal inverse, which is introduced below.

\subsubsection{Reconstruction of conductivity change and electrode move- ment}

One of the primary difficulties interpreting EIT images in clinics is the movement of electrodes from breathing or posture changes. The inaccurately modelled electrode placement introduces severe artefacts in reconstructed images. In order to solve this modelling error, Soleimani et al. (2006a) developed an algorithm to reconstruct both the conductivity change and the electrode movement simultaneously by combining conductivity changes and electrode movement reconstructions into a single inversion process. An electrode displacement vector $\Delta \mathbf{r} \in \mathbb{R}^{n_{D} n_{E}}$, where $n_{D}$ is the model dimension ( 2 or 3 for $2 \mathrm{D}$ or $3 \mathrm{D}$, respectively), is concatenated to the difference conductivity vector $\mathbf{x}_{c}$. Thus the augmented vector to be reconstructed is $\mathbf{x}=\left[\mathbf{x}_{c}^{T} \Delta \mathbf{r}^{T}\right]^{T} \in \mathbb{R}^{n_{N}+n_{D} n_{E}}$. The augmented Jacobian becomes $\mathbf{J}=\left[\mathbf{J}_{c} \mathbf{J}_{m}\right] \in \mathbb{R}^{n_{M} \times\left(n_{N}+n_{D} n_{E}\right)}$, where $\mathbf{J}_{c} \in \mathbb{R}^{n_{M} \times n_{N}}$ and $\mathbf{J}_{m} \in \mathbb{R}^{n_{M} \times n_{D} n_{E}}$ are the conductivity and electrode movement Jacobians, respectively. The overall prior matrix is $\mathbf{R}=\operatorname{diag}\left(\mathbf{R}_{c}, \mathbf{R}_{m}\right) \in \mathbb{R}^{\left(n_{N}+n_{D} n_{E}\right) \times\left(n_{N}+n_{D} n_{E}\right)}$, where $\mathbf{R}_{c} \in \mathbb{R}^{n_{N} \times n_{N}}$ and $\mathbf{R}_{m} \in \mathbb{R}^{n_{D} n_{E} \times n_{D} n_{E}}$ are conductivity and electrode movement prior matrices, respectively. Finally, the one-step reconstruction is the same as (5.3) with rebuilt $\mathbf{J}, \mathbf{x}$ and $\mathbf{R}$.

The electrode movement Jacobian $\left(\mathbf{J}_{m}\right)$ is calculated using the rank one perturbation technique (Olsen and Gopinath, 2004), as implemented by Gómez-Laberge and Adler (2007).

The prior matrix statistically describes the "desired" values of conductivity changes and electrode movements. The upper $n_{N} \times n_{N}$ part of $\Sigma_{x}^{-1}$ in (5.2) represents covariance of finite element conductivity changes, while the lower $n_{D} n_{E} \times n_{D} n_{E}$ part represents the covariance of electrode movements. By carefully establishing prior matrices for specific applications, different reconstruction performances can be obtained (Adler and Lionheart, 2006). With a similar purpose to that described in Section 5.3.1, concerning the sensitivity discrepancy, we need compensations on "overweighted" elements which have high sensitivities. The NOSER prior is used in this chapter. Suppose the calculated augmented Jacobian is $\mathbf{J}=\left[\mathbf{J}_{c} \mathbf{J}_{m}\right]$, then the $\mathbf{R}$ is built so that $[\mathbf{R}]_{i, i}=\left[\mathbf{J}^{T} \mathbf{J}\right]_{i, i}^{1 / 2}$. 


\subsubsection{Temporal one-step solver}

Adler et al. (2007) proposed a temporal image reconstruction algorithm that calculates the image at a current frame considering data from adjacent frames. This approach differs from the Kalman filter based algorithms (Vauhkonen et al., 1998a), which estimate image $\mathbf{x}_{t}$ based on measurements $\mathbf{y}_{t}$ and the previous image estimate $\mathbf{x}_{t-1}$. The temporal solver treats the estimate of the image frame sequence as a single inverse problem with a regularization prior that accounts for both spatial and temporal correlations between image elements.

\section{Temporal reconstruction}

The temporal solver considers a sequence of $2 d+1$ data frames from $t-d$ to $t+d$ around the current frame $t$. Given a vertically concatenated data frame sequence

$\tilde{\mathbf{y}}_{t}=\left[\begin{array}{lllll}\mathbf{y}_{t-d}^{T} & \ldots & \mathbf{y}_{t}^{T} & \ldots & \mathbf{y}_{t+d}^{T}\end{array}\right]^{T}$ and the corresponding concatenated image sequence $\tilde{\mathbf{x}}_{t}=$ $\left[\begin{array}{lllll}\mathbf{x}_{t-d}^{T} & \ldots & \mathbf{x}_{t}^{T} & \ldots & \mathbf{x}_{t+d}^{T}\end{array}\right]^{T}$, the direct temporal forward model is rewritten from (5.1) as

$$
\tilde{\mathbf{y}}_{t}=\tilde{\mathbf{J}} \tilde{\mathbf{x}}_{t}+\tilde{\mathbf{n}}
$$

where $\tilde{\mathbf{n}}=\left[\begin{array}{lllll}\mathbf{n}_{t-d}^{T} & \ldots & \mathbf{n}_{t}^{T} & \ldots & \mathbf{n}_{t+d}^{T}\end{array}\right]^{T}$. We consider that $\mathbf{J}$ is time invariant. Thus, $\tilde{\mathbf{J}}=\mathbf{I} \otimes \mathbf{J}$, where the identity matrix $\mathbf{I}$ is of size $2 d+1$, and $\otimes$ is the Kronecker product.

There exists an inter-frame correlation between two images temporally close to each other. As images become further separated in time, the correlation decreases; for a separation of $\delta$, the correlation is $\exp (-|\delta| / \gamma)$, where $\gamma$ is the temporal exponential decay factor in units of frames. Frames with a large time difference $|\delta|>d$, are considered independent. The one-step inverse (5.4) then becomes

$$
\tilde{\mathbf{B}}=\tilde{\mathbf{P}} \tilde{\mathbf{J}}^{T}\left(\tilde{\mathbf{J}} \tilde{\mathbf{P}} \tilde{\mathbf{J}}^{T}+\lambda^{2} \tilde{\mathbf{V}}\right)^{-1},
$$

where $\tilde{\mathbf{V}}=\mathbf{I} \otimes \mathbf{V}$. Also $\tilde{\mathbf{P}}=\boldsymbol{\Gamma} \otimes \mathbf{P}$, where $\boldsymbol{\Gamma}$ is the temporal weight matrix of an image sequence $\tilde{\mathbf{x}}$ and is defined to have the form

$$
[\boldsymbol{\Gamma}]_{i, j}=\exp \left(-\frac{|i-j|}{\gamma}\right) \quad i, j=-d, \ldots, d
$$

From (5.6) and (5.7),

$$
\tilde{\mathbf{B}}=\left[\mathbf{\Gamma} \otimes\left(\mathbf{P} \mathbf{J}^{T}\right)\right]\left[\mathbf{\Gamma} \otimes\left(\mathbf{J P J}^{T}\right)+\lambda^{2}(\mathbf{I} \otimes \mathbf{V})\right]^{-1} .
$$

Given $\tilde{\mathbf{B}}$, the one-step solution $\hat{\mathbf{x}}_{t}$ for the current image is rewritten as

$$
\hat{\mathbf{x}}_{t}=\tilde{\mathbf{B}}_{0} \tilde{\mathbf{y}}_{t}
$$

where $\tilde{\mathbf{B}}_{0}$ occupies the rows $n_{M} \times d+1, \ldots, n_{M} \times(d+1)$ of $\tilde{\mathbf{B}}$. 


\section{Parameter selection}

The $\gamma$ may be considered a hyperparameter of the system: it depends on the data acquisition frame rate, the speed of underlying conductivity changes and the system noise level. The estimation of $\gamma$ can be addressed as a method of "kernel smoothing" (Fahrmeir and Tutz, 1994). The object function to be minimized can be averaged squared error, mean average squared error, or average predictive squared error, etc.

For details of calculating $\gamma$, please refer to Chapter 4.3.2 "Parameter selection".

\subsubsection{Temporal reconstruction of conductivity change and elec- trode movement}

The temporal solver for reconstruction of both conductivity change and electrode movement is formulated in terms of a regularized one-step inverse (5.8), in which the Jacobian $\mathbf{J}$ is rebuilt as $\left[\mathbf{J}_{c} \mathbf{J}_{m}\right]$ and

$$
\tilde{\mathbf{J}}=\mathbf{I} \otimes\left[\mathbf{J}_{c} \mathbf{J}_{m}\right] .
$$

The augmented prior matrix is computed as NOSER prior from the precalculated Jacobian and

$$
\tilde{\mathbf{P}}=\Gamma \otimes\left[\mathbf{J}^{T} \mathbf{J}\right]_{i, i}^{-1 / 2} .
$$

The inverse is further processed in terms of an augmented image $\tilde{\mathbf{x}}$ and measurement vector $\tilde{\mathbf{y}}(5.9)$, which concatenate the values from $d$ previous and $d$ future frames.

\subsubsection{Method: experiments}

Data from numerical simulations, a saline phantom measurement and in vivo human measurement were used to test these algorithms proposed.

\section{Numerical simulation}

Numerical simulation data were obtained from a 2D FEM model with 5184 elements, with homogeneous conductivity $\sigma_{h}=1$. As illustrated in Figure 5.1(a), A unit radius circular model with 16 electrodes was built and an adjacent stimulation and measurement pattern was applied. In this model a conductive $\left(1.2 \times \sigma_{h}\right)$ spherical object with 0.05 unit radius rotated clockwise along a trajectory that had a radius of $2 / 3$ unit, Two hundred frames were taken per movement cycle. A distortion (horizontal compression and vertical elongation) 
was applied with distortion amplitudes gradually increased from 0 at the first frame to $1 \%$ of the model diameter at the end ( $200^{\text {th }}$ frame).

\section{Phantom measurement}

The saline phantom is a plastic cylindrical tank with $30 \mathrm{~cm}$ diameter and $30 \mathrm{~cm}$ height, being filled with $0.9 \%$ saline solution to the $20 \mathrm{~cm}$ height. Sixteen stainless steel electrodes were equidistantly placed around the circumference at a vertical position of $10 \mathrm{~cm}$. EIT data were acquired using the Goe-MF II EIT system (Viasys Healthcare, Höchberg, Germany) using an adjacent stimulation and measurement pattern. First, data of a homogeneous background $\mathbf{y}_{h}$ were acquired and processed by ensemble averaging; then two small non-conductive spherical objects of $2 \mathrm{~cm}$ radius were statically suspended at the same level of the electrode plane at positions $(0,7 \mathrm{~cm})$ and $(7 \mathrm{~cm}, 0)$ as illustrated in Figure 5.1(b). A compression was applied on the top of the phantom along the $x$-axis so that the cross section of the tank became an ellipse with a minor axis of $25 \mathrm{~cm}$. Since the electrode plane was at $1 / 3$ of the tank height, the maximum movement of electrodes was about $1.7 \mathrm{~cm}$. This phantom was gradually compressed during the first 6 seconds and held with elliptical shape afterwards.

\section{In vivo human measurement}

EIT data were acquired from a healthy adult using the Goe-MF II EIT system (Viasys Healthcare, Höchberg, Germany). Adjacent stimulation and measurement patterns were applied. The data acquisition speed was $13 \mathrm{fps}$. Sixteen $3 M^{T M}$ Red-Dot $\mathrm{Ag} / \mathrm{AgCl}$ electrodes were equidistantly attached around the thoracic circumference at a horizontal plane that was $1 \mathrm{~cm}$ under the nipple line. The $1^{\text {st }}$ Electrode was in the centre of the sternum. Other electrodes were subsequently placed towards the subject's right side so that the $5^{\text {th }}$ electrode was under the right armpit, the $9^{\text {th }}$ electrode on the spine and the $13^{\text {th }}$ electrode under the left armpit. A $17^{\text {th }}$ electrode was attached at lower right waist as reference/ground. All measurements were taken while the subject was standing and conducting deep breaths to total lung capacity.

\subsection{Results}

The forward and inverse calculations used the EIDORS software (Adler and Lionheart, 2006). The numerical and phantom data were reconstructed on a circular 576 element 


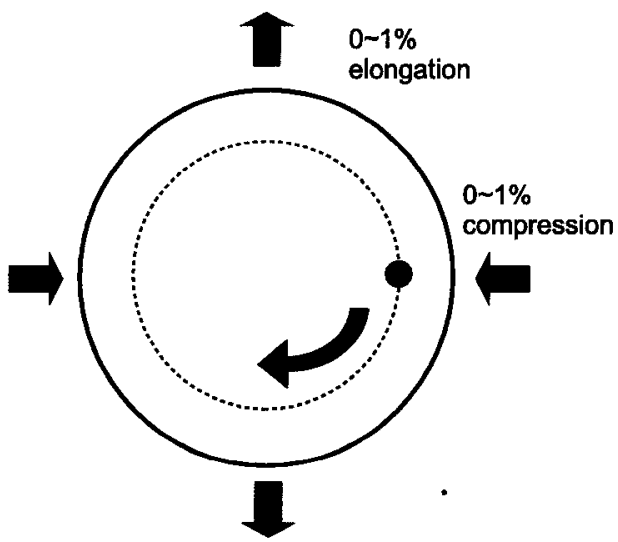

(a)

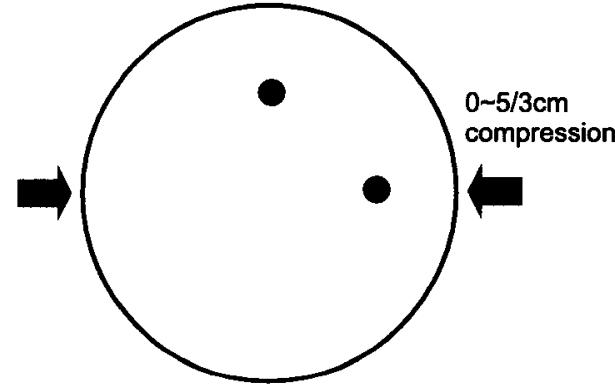

(b)

Figure 5.1: Illustration of 2D numerical model simulation and saline phantom measurement. Green arrows indicate directions of boundary distortion. (a) one conductive target rotates clockwise (trajectory as dashed circle). Distortion amplitude increases linearly from 0 to $1 \%$ of model diameter. Background conductivity $\sigma_{h}=1$ and the target conductivity is $1.2 \times \sigma_{h}$. The illustrated target position is the start/final position of one full cycle. (b) two non-conductive targets statically suspended in a saline phantom at the level of the electrode plane. The phantom diameter is $30 \mathrm{~cm}$, the maximum distortion of the boundary is $5 / 3 \mathrm{~cm}$.

model and the in vivo data was on a $2 \mathrm{D}$ thoracic 576 element model. For the numerical simulation, the choice of 576 elements for the inverse mesh was to differ from the simulation model to avoid the inverse crime. Gaussian white noise was added to numerical simulated data with noise level $S N R$, defined as $\overline{\mathbf{y}} / \sigma_{\mathbf{n}}$, where $\sigma_{\mathbf{n}}$ was the standard deviation of the added noise and $\overline{\mathbf{y}}$ was the mean value of the difference signal. Different random seeds were tested and showed similar results. Four algorithms were evaluated with different regularization methods: 1) GN solver (Sec.5.3.1); 2) temporal solver (Sec.5.3.3); 3) electrode movement solver (Sec.5.3.2); and 4) temporal and electrode movement solver (Sec.5.3.4).

To better understand the effect of the model distortion on reconstructed image, we used the traditional GN solver to calculate images of a distorted homogeneous medium (Figure 5.2, left). The $2 \mathrm{D}$ model is divided into four regions, regions 1 and 3 are compressed horizontally and elongated vertically. This distortion can be understood as a conductor with shorter length and larger cross section; therefore, the conductance is increased. This is equivalent to the situation of increased conductivity with unchanged geometry (Figure 5.2 , middle). Without considering any model geometry variations, the reconstructed image shows regions 1 and 3 as positive conductivity changes (red) (Figure 5.2, right). Inversely, 
regions 2 and 4 are represented as negative conductivity changes (blue). These effects are named "deformation artefacts". As illustrated in Figure 5.3: at the $11^{\text {th }}$ frame (fn=11,
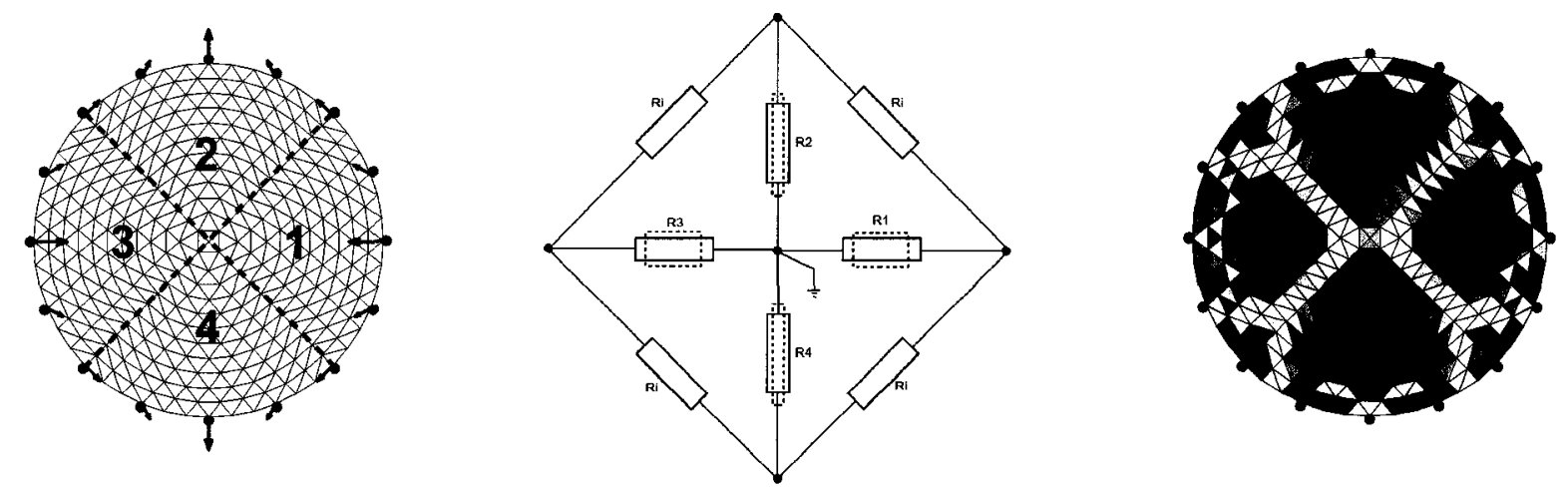

Figure 5.2: Effect of boundary distortion on EIT reconstruction.

left: a homogeneous medium with a distorted boundary. Four regions are defined to identify different effects.

middle: the simplified circuit model of the medium. $R_{1, \ldots, 4}$ represent resistances of regions $1, \ldots, 4$, respectively. $R_{i}$ are intermediate resistances between two regions. In regions 1 and 3 , the boundary distortion can be regarded as shortened and widened resistors (dashed blocks), and in regions 2 and 4 as elongated and thinned resistors.

right: image reconstructed using GN method with an assumed static boundary $(\lambda=0.4$, noisefree).

distortion amplitude is $0.05 \%$ of the model diameter), although temporal solvers show better target resolution, all methods identify target successfully due to the small model deformation. With increasing model deformation $(\mathrm{fn}=61,0.3 \%$ distortion; $\mathrm{fn}=111,0.55 \%$ distortion), the GN and temporal solvers failed to identify the target due to severe distortion artefacts. The electrode movement solver still shows the target, although artefacts emerge. Further deformation ( $\mathrm{fn}=161,0.8 \%$ distortion) makes the electrode movement solver incapable of recognizing target due to severe artefacts. Compared with other solvers, the temporal and electrode movement solver is much more robust against the "deformation artefacts". Illustrated in Figure 5.4, a similar conclusion can be drawn from phantom data reconstructions. With gradually increased deformation $(0 \sim 1.7 \mathrm{~cm}$, approximately from $\mathrm{fn}=1$ to $\mathrm{fn}=75$ ), the temporal and electrode movement solver shows the best artefact robustness. The gradual disappearance of the target at the position $(7,0)$ illustrates the "deformation artefacts": the target is gradually obscured by the positive artefacts introduced by boundary deformation. In order to test the applicability to in vivo measurements, 

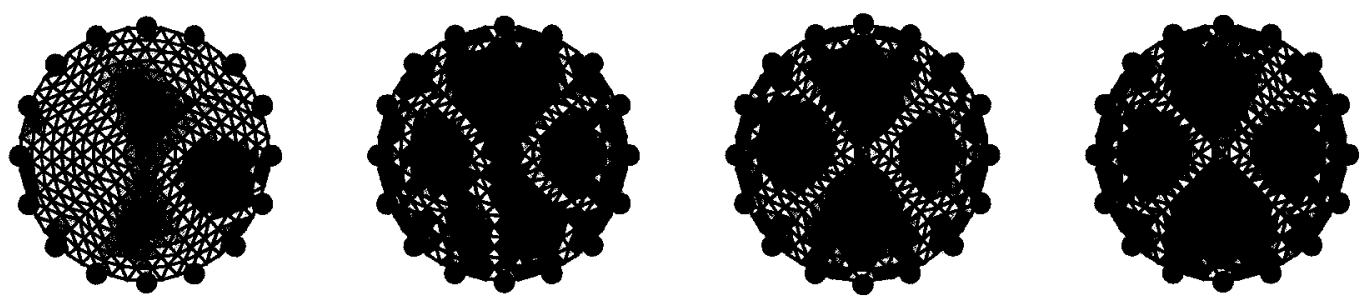

(a)
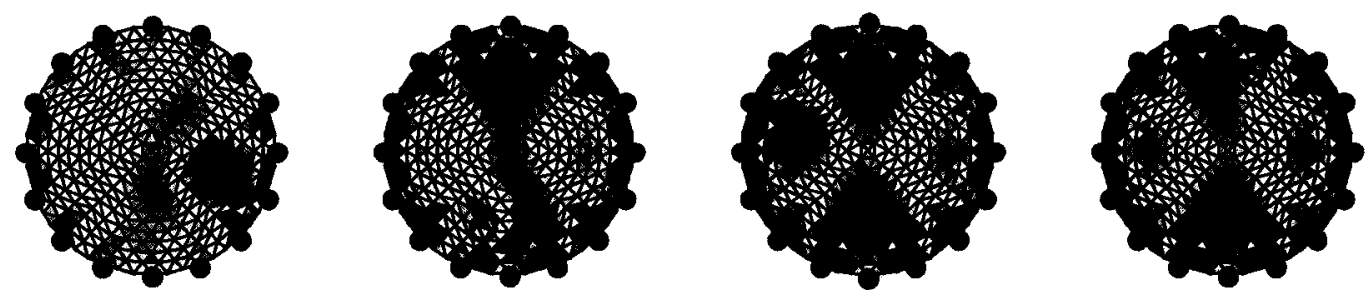

(b)
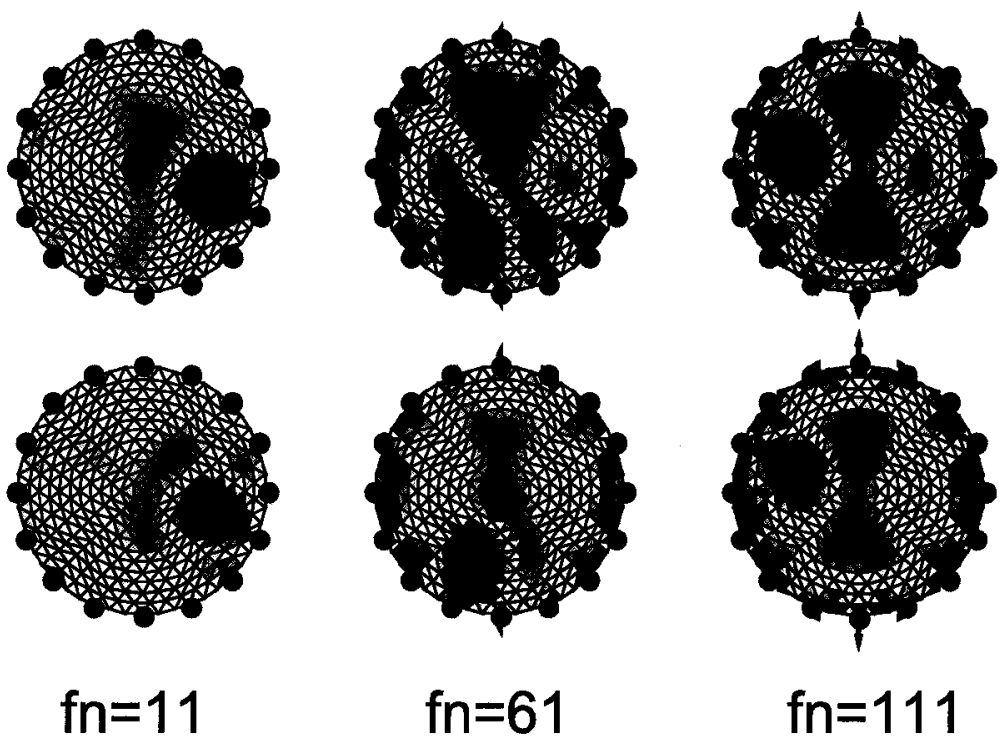

$\mathrm{fn}=61$
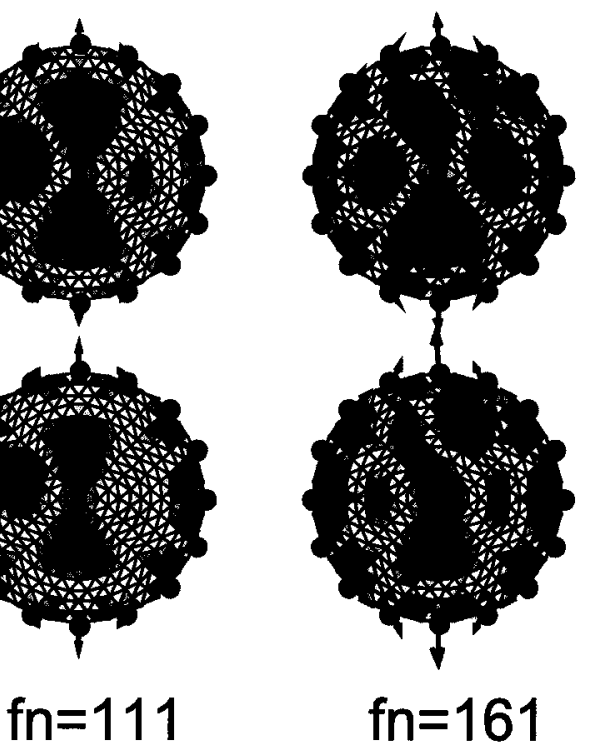

(c)

(d)

Figure 5.3: Images reconstructed from simulated data (using $\lambda=0.4, S N R=10$ ). (a) GN solver; (b) Temporal solver; (c) Electrode movement solver; (d) Temporal and Electrode movement solver. From left to right, the target rotated clockwise (simulated target positions were shown by black circles); the boundary was increasingly distorted and arrows indicate amplitudes and directions of reconstructed electrode movements. Arrow amplitudes are scaled by 20 .

a thirty-second frame sequence was acquired (the frame rate was $13 \mathrm{fps}$, thus 390 frames in total) for a deep-breathing human subject. Data were reconstructed by the temporal and electrode movement solver (Figure 5.5). The reconstructed image sequence was chosen from the end inspiration to the end expiration. The reference data $\left(\mathbf{v}_{0}\right)$ were chosen as the 

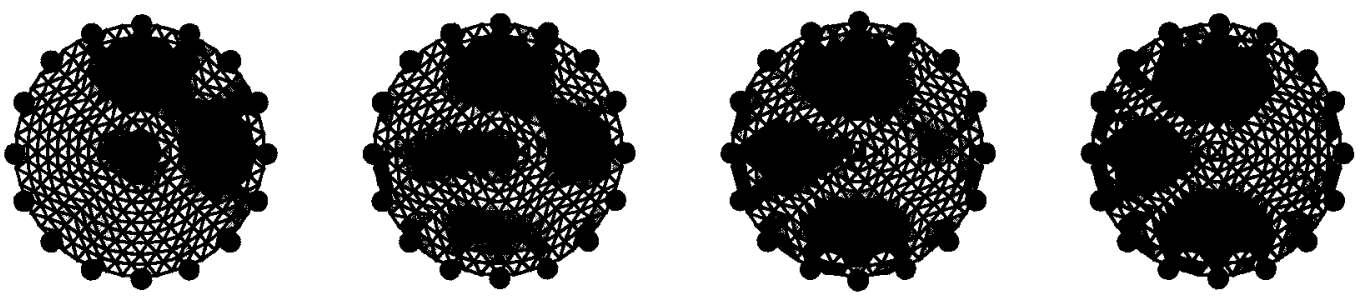

(a)
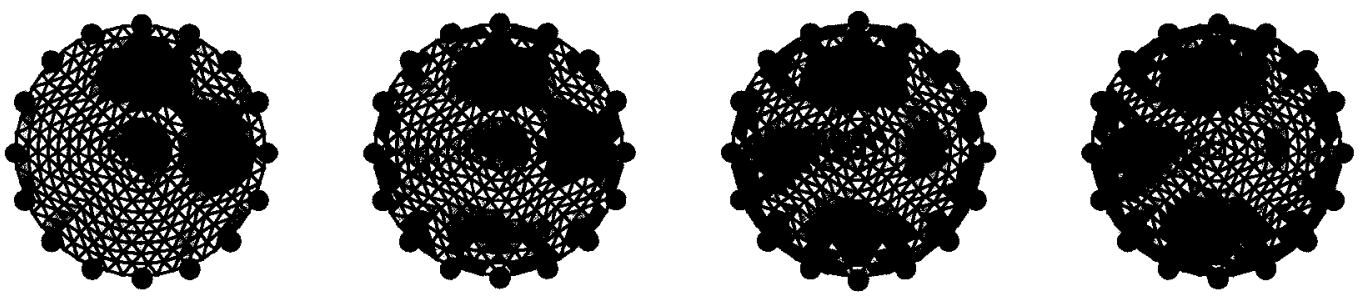

(b)
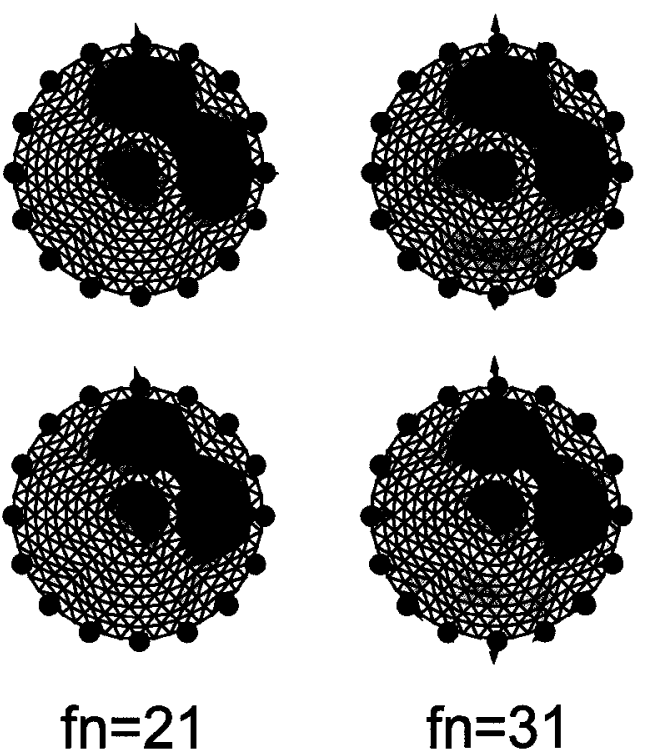

$\mathrm{fn}=31$
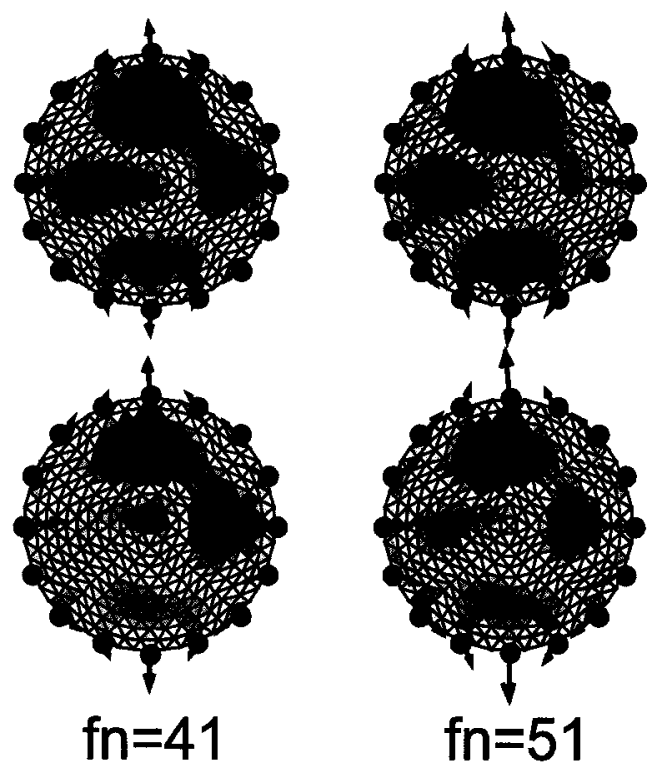

(c)

(d)

Figure 5.4: Images reconstructed from different solvers using measured data from a saline phantom (using $\lambda=0.4$ ). (a) GN solver; (b) Temporal solver; (c) Electrode movement solver; (d) Temporal and Electrode movement solver. From left to right, frames were taken at 21, 31, 41 and 51, corresponding to increasing boundary distortion. Arrows indicate amplitudes and directions of boundary distortion. Arrow amplitudes are scaled by 10 .

average of the whole data set. At the end inspiration when $t=17.8 \mathrm{sec}$, the lungs showed conductivity decrease in blue (compared with the reference); during expiration, conductivity gradually increased and, after crossing the reference $\left(\mathbf{x}_{0}\right)$, the lungs regions showed increased conductivity (in red); they finally reached the end of expiration at $t=22.8 \mathrm{sec}$ 
when lungs remained the residue volume and the image showed the highest conductivity.
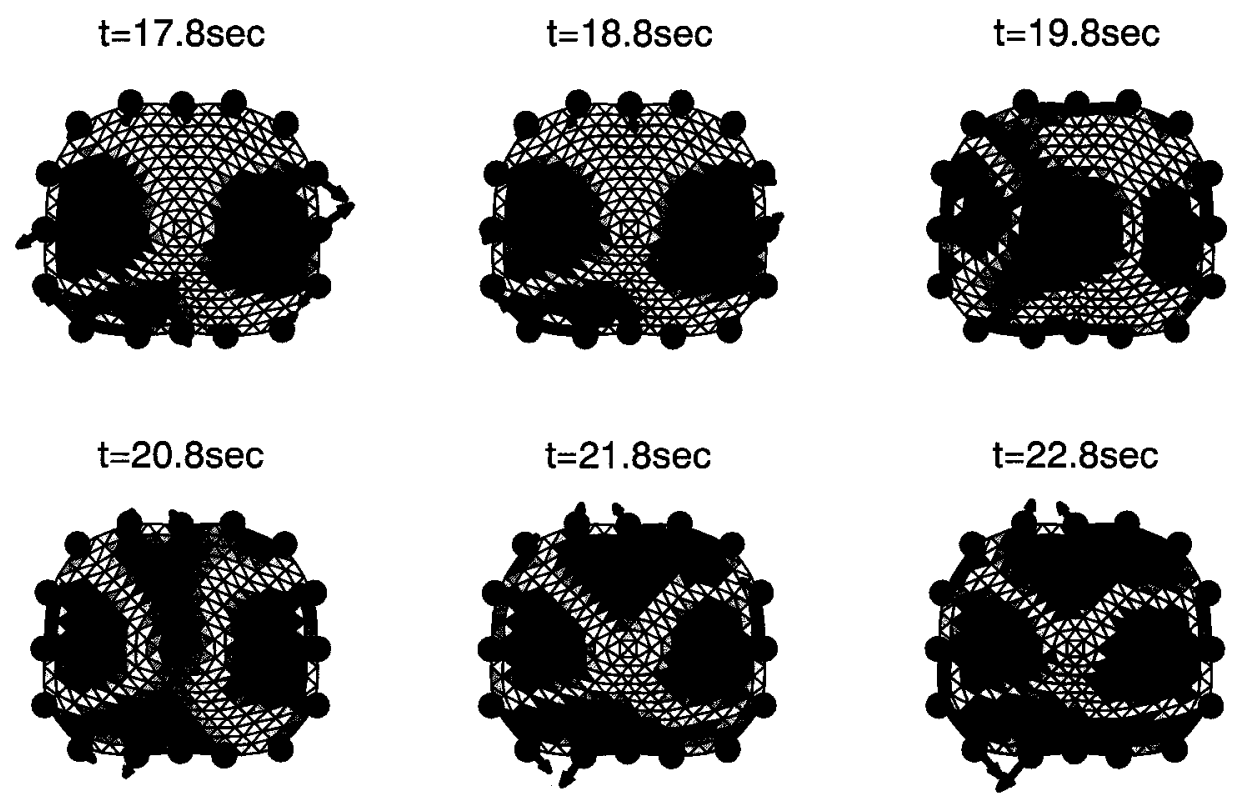

Figure 5.5: Pulmonary monitoring of a human subject (maximum expiration) using $\lambda=2$. Anterior is at image top; left is at image left. Arrows show movement directions and amplitudes that are exaggerated by 30 . The reference signal was calculated as the average over whole data set.

\subsection{Discussion}

In this chapter, we propose an image processing algorithm to help address two issues in EIT: uncertainty in boundary movement and noise in reconstructed images. Images of the conductivity change and electrode movement are calculated from sequences of EIT data around the current frame. The temporal reconstruction proposed directly formulates both the reconstructed conductivity change and electrode movement in terms of a single regularized inverse, based on a priori models that adjacent images and boundary shapes from a fast measurement system are highly correlated. This method takes advantage of correlations that occur because the conductivity changes and boundary movements happen more slowly than the data acquisition.

In this chapter, one important assumption is that the Jacobian is time-invariant. This is a core assumption in time-difference EIT. The model is linearized by taking a derivative 
at a reference point. Simulations suggest that the changes in the Jacobian are much less significant than any general inaccuracies in the Jacobian used due to inadequate knowledge of the initial geometry and conductivity distribution.

This result is based on our previous work (Adler et al., 2007; Soleimani et al., 2006a); the combined solver of temporal inverse and electrode movement reconstruction uses temporal reconstruction method to calculate both the conductivity change and electrode movement from difference EIT data. The novel results demonstrated in this chapter are to show significant improvements in noise performance and artefact resistance. The algorithm performed well in simulated and phantom in comparison to reconstructions which consider only the conductivity change, and in comparison to our previous results for electrode movement. Considering the reconstructed images and electrode movement from in vivo human data, this method shows potential to be used in real time monitoring of lung ventilation. 


\section{Chapter 6}

\section{Electrical Impedance Tomography Reconstruction Using $\ell_{1}$ Norm on Data and Image Terms}

\subsection{Summary}

This chapter is based on the paper-Electrical Impedance Tomography Reconstruction Using $\ell_{1}$ Norm on Data and Image Terms,30th Annual International Conference of the IEEE EMBC, Vancouver, Canada, August, 2008. Authors are Tao Dai and Andy Adler. This work is in progress and will be submitted as a peer-reviewed publication.

Electrical Impedance Tomography (EIT) calculates the internal conductivity distribution within a body from current simulation and voltage measurements on the body surface. Two main technical difficulties of EIT are its low spatial resolution and sensitivity to measurement errors. Image reconstruction using $\ell_{1}$ norms allows addressing both difficulties, in comparison to traditional reconstruction using $\ell_{2}$ norms. An $\ell_{1}$ norm on the data residue term reduces the sensitivity to measurement errors, while the $\ell_{1}$ norm on the image prior reduces edge blurring. This chapter proposes and tests a general lagged diffusivity type iterative method for EIT reconstructions. $\ell_{1}$ and $\ell_{2}$ minimizations can be flexibly chosen on the data residue and/or image prior parts. Results show the flexibility of the algorithm and the merits of the $\ell_{1}$ solution. 


\subsection{Introduction}

Electrical Impedance Tomography (EIT) images the impedance distribution within a body from electrical stimulation and measurements on the body surface. One of key limitations of EIT is its relatively poor image resolution, which, for 16 electrodes is less than $10 \%$ of the body diameter. EIT is a soft field tomography modality, due to the diffusive propagation of electrical current. Thus, the reconstruction of an internal conductivity distribution from boundary data is severely ill-conditioned (Lionheart et al., 2005). In order to calculate a "reasonable" image, regularization techniques are required. Such regularized image reconstructions can be statistically formulated in terms of a priori information about image element values and the correlations among them. These correlations are often expressed as generalized Tikhonov regularization; the zeroth order Tikhonov (Vauhkonen et al., 1998b), discrete Laplacian filter (Polydorides and Lionheart, 2002) and weighted diagonal (NOSER) priors (Cheney et al., 1990; Graham and Adler, 2007), etc. Another limitation to the quality of EIT images is measurement errors, which arise from multiple sources, such as RF coupling onto signal wires, electrode malfunction, and subject movement. While it is common to model such measurement noise as Gaussian, such noise sources introduce many more outliers than the the Gaussian model would predict. Most image reconstruction algorithms for EIT search for an image solution, $\hat{\mathbf{x}}$, which minimizes an error expression based on the $\ell_{2}$ norm, e.g., one-step GN method. However, these algorithms are known to blur image regions and be sensitive to data outliers.

It is widely recognized that the Total Variation (TV) ( $\ell_{1}$ norm of image spatial gradient) regularization is good at recovering discontinuities in the image while the Least Squares (LS, or $\ell_{2}$ norm) solution is prone to smooth out edges. This is because penalty terms using $\ell_{2}$ norm penalize smooth transitions less than sharp transitions, while $\ell_{1}$ norms penalize only the transition amplitude, and not its slope. Similarly, the $\ell_{2}$ penalty for a data outlier is larger (the difference is squared) than for the $\ell_{1}$. This means the $\ell_{1}$ solution is less perturbed by outliers. However, the $\ell_{1}$ solution involves the minimization of a nondifferentiable objective function, and thus cannot be efficiently solved by the traditional optimization methods that minimize a differentiable objective function such as the Steepest Decent and GN method.

In this chapter we propose an image reconstruction algorithm based on the laggeddiffusivity method in which the $\ell_{1}$ norm is applied to both the image prior and data fidelity term. This preserves image edges and provides enhanced resistance against data errors. This algorithm has a general iterative structure which enables flexibly choosing 
different norm strategies, and termination criteria.

\subsection{Methods}

An EIT system with $n_{E}$ electrodes is considered. Electrodes are applied to a body in a single plane and adjacent current stimulation and voltage measurement are performed. $n_{E}$ current stimulation patterns are sequentially applied and $n_{V}$ differential measurements are made for each stimulation. Difference EIT calculates difference data $\mathbf{y}=\mathbf{v}_{2}-\mathbf{v}_{1}$, where $\mathbf{y}, \mathbf{v} \in \mathbb{R}^{n_{M}}, n_{M}=n_{E} \times n_{V}$, and $\mathbf{v}_{1}$ and $\mathbf{v}_{2}$ are the vectors or measurements before and after a conductivity change of interest. To improve precision, $\mathbf{v}_{1}$ is typically averaged over many data frames, at a time when the conductivity distribution may be assumed to be stable; thus. $\mathbf{v}_{1}$ is assumed noise free.

The model under investigation is a circular finite element model (FEM) which has $n_{N}$ piecewise elements represented by a vector $\sigma \in \mathbb{R}^{n_{N}}$. Difference EIT calculates a vector of conductivity change, $\mathbf{x}=\sigma_{2}-\sigma_{1}$ between the present conductivity distribution, $\sigma_{2}$, and the reference measurement, $\sigma_{1}$. In this paragraph, $\sigma$ represents conductivity; elsewhere in this chapter, $\sigma$ is the standard deviation. For small variations around $\sigma_{1}$, the relationship between $\mathbf{x}$ and $\mathbf{y}$ can be linearized as:

$$
\mathbf{y}=\mathbf{J x}+\mathbf{n}
$$

where $\mathbf{J} \in \mathbb{R}^{n_{M} \times n_{N}}$ is the Jacobian or sensitivity matrix; $\mathbf{n} \in \mathbb{R}^{n_{M}}$ is the measurement noise which is assumed to be uncorrelated white Gaussian. $\mathbf{J}$ is calculated from the FEM

as $\mathbf{J}_{i j}=\left.\frac{\partial \mathbf{y}_{i}}{\partial \mathbf{x}_{j}}\right|_{\sigma_{1}}$. This system is underdetermined since $n_{N}>n_{M}$, and regularization techniques are needed to calculate a conductivity change estimate, $\hat{\mathbf{x}}$, which is faithful to both the measurements, $\mathbf{y}$, and to a priori constraints on a "reasonable" image.

\subsubsection{Least Squares $\left(\ell_{2}\right.$ norm) solution}

The LS solution of (6.1) can be obtained using GN method which seeks a solution $\hat{\mathbf{x}}$ by minimizing

$$
\|\mathbf{y}-\mathbf{J} \mathbf{x}\|_{\Sigma_{n}^{-1}}^{2}+\left\|\mathbf{x}-\mathbf{x}_{0}\right\|_{\Sigma_{x}^{-1}}^{2}
$$

where $\|\cdot\|^{2}$ is the $\ell_{2}$ norm, and the norm subscript is the weight matrix, such that $\|\mathbf{x}\|_{\mathbf{w}}^{2}=$ $\sum_{i} \sum_{j} \mathbf{x}_{i} \mathbf{W}_{i j} \mathbf{x}_{j} . \quad \mathbf{x}_{0}$ is the a priori mean conductivity change. $\boldsymbol{\Sigma}_{n} \in \mathbb{R}^{n_{M} \times n_{M}}$ is the covariance matrix of the measurement noise $\mathbf{n}$. Since $\mathbf{n}$ is uncorrelated, $\boldsymbol{\Sigma}_{n}$ is a diagonal matrix with $\left[\boldsymbol{\Sigma}_{n}\right]_{i, i}=\sigma_{i}^{2}$, where $\sigma_{i}^{2}$ is the noise variance at measurement $i . \boldsymbol{\Sigma}_{x} \in \mathbb{R}^{n_{N} \times n_{N}}$ 
is the expected image covariance. Let $\mathbf{W}=\sigma_{n}^{2} \boldsymbol{\Sigma}_{n}^{-1}$ and $\mathbf{R}=\sigma_{x}^{2} \boldsymbol{\Sigma}_{x}^{-1} . \mathbf{W}$ and $\mathbf{R}$ are heuristically determined a priori. Here $\sigma_{n}$ is the average measurement noise amplitude and $\sigma_{x}$ is the a priori amplitude of conductivity change.

By solving (6.2) and defining a hyperparameter $\lambda=\sigma_{n} / \sigma_{x}$, a linearized, one-step inverse solution is obtained Adler et al. (2007)

$$
\hat{\mathbf{x}}=\left(\mathbf{J}^{T} \mathbf{W} \mathbf{J}+\lambda^{2} \mathbf{R}\right)^{-1} \mathbf{J}^{T} \mathbf{W} \mathbf{y}=\mathbf{B} \mathbf{y}
$$

where $\mathbf{B}=\left(\mathbf{J}^{T} \mathbf{W} \mathbf{J}+\lambda^{2} \mathbf{R}\right)^{-1} \mathbf{J}^{T} \mathbf{W}$ is the linear, one-step inverse. $\lambda$ controls the trade-off between resolution and noise attenuation in the reconstructed image.

If image elements are assumed to be independent with identical expected magnitude, $\mathbf{R}$ becomes an identity matrix, $\mathbf{I}$, and (6.3) uses zeroth-order Tikhonov regularization. For EIT, such solutions tend to push reconstructed noise toward the boundary, since the measured data is much more sensitive to boundary image elements. Instead, $\mathbf{R}$ may be scaled with the sensitivity of each element, so that $\mathbf{R}$ is a diagonal matrix with elements $[\mathbf{R}]_{i, i}=\left[\mathbf{J}^{T} \mathbf{J}\right]_{i, i}^{p}$. This is the NOSER prior (Cheney et al., 1990) for an exponent $p$, where $p \in[0,1]$. The TV prior is the discretization of the gradient operator. the TV of a $2 \mathrm{D}$ image is the sum of the variation across each mesh edges, with each edge weighted by its length (Borsic et al., 2007). In this chapter, the TV prior is used to calculate the matrix R.

\subsection{2 $\ell_{1}$ norm solution}

When applied to the image prior $\left\|\mathbf{x}-\mathbf{x}_{0}\right\|, \ell_{2}$ norm solutions tend to give "smoothed" images, because the prior applies strong penalties to edges. However, strong edges are physiologically realistic, and are desired in the images. Although edge blur can be decreased using a small hyperparameter, $\lambda$, this dramatically decreases noise performance. Another method is to carefully define a prior with a priori knowledge of edge locations (Kaipio et al., 1999). However, this approach can result in image artefacts that appear plausible, and thus hard to detect (e.g., Adler and Lionheart (2006)), if the prior information is too detailed, but does not describe the actual image.

The Total Variation (TV) of the $\ell_{1}$ norm is known to work well to preserve intrinsic edges in original images. However, $\ell_{1}$ norm solutions are difficult because the objective function is non-differentiable and cannot be efficiently solved with traditional linearization techniques. Minimization of functions of TV norms normally uses iterative methods. The primal dual interior point method (PD-IPM) was proposed (Chan et al., 1999) to solve the TV 
minimization problem by removing the singularity points which caused non-differentiability before applying the linearization method. A mixed norm TV solution (Borsic et al., 2007) for EIT was formulated as:

$$
\hat{\mathbf{x}}=\underset{\mathbf{x}}{\operatorname{argmin}}\|\mathbf{y}-\mathbf{J} \mathbf{x}\|_{2}^{2}+\left\|\mathbf{x}-\mathbf{x}_{0}\right\|^{1}
$$

where $\|\cdot\|^{2}$ is the $\ell_{2}$ norm and $\|\cdot\|^{1}$ is the $\ell_{1}$ norm weighted by the TV prior.

Another attractive property of $\ell_{1}$ solution is its resistance to data outliers. For the data residue term, $\mathbf{y}-\mathbf{J x}$, the $\ell_{2}$ norm is highly sensitive to data outliers, because it assumes a Gaussian distribution, which over weights the significance of large outliers. The $\ell_{1}$ solution is inherently more robust against outliers in measurements because it does not square each measurement misfit. This property of $\ell_{1}$ regularization is promising, especially for EIT, because measurement errors constitute one of primary technical obstacles of clinical EIT, where erroneous electrodes introduce severe artefacts (Asfaw and Adler, 2005).

We propose applying $\ell_{1}$ regularization to both the data residual and the image prior; the optimization problem becomes

$$
\hat{\mathbf{x}}=\underset{\mathbf{x}}{\operatorname{argmin}}\|\mathbf{y}-\mathbf{J x}\|^{1}+\left\|\mathbf{x}-\mathbf{x}_{0}\right\|^{1}
$$

A well known algorithm to the sum of $\ell_{1}$ norms is Iteratively Reweighted Least Squares (IRLS) (Scales et al., 1988). The IRLS method iteratively solves a weighted least squares problem which begins as an $\ell_{2}$ norm, and converges to the $\ell_{1}$ norm solution.

\subsubsection{Generalized $\ell_{1}$ and $\ell_{2}$ regularization with iterative method}

A weighted and regularized inverse may be generally formulated as

$$
\hat{\mathbf{x}}=\underset{\mathbf{x}}{\operatorname{argmin}}\|\mathbf{y}-\mathbf{J} \mathbf{x}\|_{\mathbf{\Sigma}_{n}^{-1}}^{p_{n}}+\left\|\mathbf{x}-\mathbf{x}_{0}\right\|_{\Sigma_{x}^{-1}}^{p_{x}}
$$

where $p_{n}$ and $p_{x}$ are the data and image norms and must be $\geq 1$ for stability. The norm subscript is the weight matrix, such that $\|\mathbf{x}\|_{\mathbf{w}}^{p}=\sum_{i} \sum_{j} \mathbf{x}_{i}^{p / 2} \mathbf{W}_{i j} \mathbf{x}_{j}^{p / 2}$. A weighted $p$ norm With $p_{n}=p_{x}=2$, both term use $\ell_{2}$ norms, equivalent to (6.2), and denoted $\ell_{2}-\ell_{2}$. With $p_{n}=2, p_{x}=1$ it models the implementation of $(6.4)$, and is denoted $\ell_{2}-\ell_{1}$. In this chapter, a general iterative algorithm for (6.6) is developed, which allows flexible choice of combinations of norms by simply choosing difference $p_{n}$ and $p_{x}$. A similar $\ell_{k}$ norm choosing method can be found in works of Cetin and Karl (2001). 
(6.6) is reformulated in quadratic forms:

$$
\begin{array}{r}
\hat{\mathbf{x}}=\underset{\mathbf{x}}{\operatorname{argmin}}(\mathbf{y}-\mathbf{J} \mathbf{x})^{t} \mathbf{D}_{n}^{t} \boldsymbol{\Sigma}_{n}^{-1} \mathbf{D}_{n}(\mathbf{y}-\mathbf{J} \mathbf{x})+ \\
\left(\mathbf{x}-\mathbf{x}_{0}\right)^{t} \mathbf{D}_{x}^{t} \boldsymbol{\Sigma}_{x}^{-1} \mathbf{D}_{x}\left(\mathbf{x}-\mathbf{x}_{0}\right)
\end{array}
$$

where $\mathbf{D}_{n}$ is a diagonal matrix in which

$$
\left[\mathbf{D}_{n}\right]_{i, i}=\left([|\mathbf{y}-\mathbf{J x}|]_{i}\right)^{\frac{1}{2} p_{n}-1}
$$

here $|\cdot|$ is the absolute value. Similarly, $\mathbf{D}_{x}$ is a diagonal matrix with

$$
\left[\mathbf{D}_{x}\right]_{i, i}=\left(\left[\left|\mathbf{x}-\mathbf{x}_{0}\right|\right]_{i}\right)^{\frac{1}{2} p_{x}-1}
$$

Note that for $p_{n}=2$ or $p_{x}=2, \mathbf{D}_{n}$ or $\mathbf{D}_{x}$ will be the identity matrix. When $p_{n}=1$ or $p_{x}=1,\left[\mathbf{D}_{n}\right]_{i, i}=\left([|\mathbf{y}-\mathbf{J} \mathbf{x}|]_{i}\right)^{-\frac{1}{2}}$ or $\left[\mathbf{D}_{x}\right]_{i, i}=\left(\left[\left|\mathbf{x}-\mathbf{x}_{0}\right|\right]_{i}\right)^{-\frac{1}{2}}$. In order to remove singular points where $[|\mathbf{y}-\mathbf{J} \mathbf{x}|]_{i}$ or $\left[\left|\mathbf{x}-\mathbf{x}_{0}\right|\right]_{i}$ equal zero, (6.8) and (6.9) are modified as follows

$$
\begin{aligned}
& {\left[\mathbf{D}_{n}\right]_{i, i}=\left([|\mathbf{y}-\mathbf{J} \mathbf{x}|]_{i}+\beta\right)^{\frac{1}{2} p_{n}-1}} \\
& {\left[\mathbf{D}_{x}\right]_{i, i}=\left(\left[\left|\mathbf{x}-\mathbf{x}_{0}\right|\right]_{i}+\beta\right)^{\frac{1}{2} p_{x}-1}}
\end{aligned}
$$

where $\beta$ is a small positive scalar.

This formulation leads to an iterative update expression for calculation of $\hat{\mathbf{x}}$; the $k+1$ iteration $\hat{\mathbf{x}}^{(k+1)}$ is calculated from $\hat{\mathbf{x}}^{(k)}$ using

$$
\begin{array}{r}
\hat{\mathbf{x}}^{(k+1)}=\mathbf{x}^{(k)}+\left(\mathbf{J}^{t} \mathbf{W}\left(\mathbf{x}^{(k)}\right) \mathbf{J}+\lambda^{2} \mathbf{R}\left(\mathbf{x}^{(k)}\right)\right)^{-1} \\
\mathbf{J}^{t} \mathbf{W}\left(\mathbf{x}^{(k)}\right)\left(\mathbf{y}-\mathbf{J} \mathbf{x}^{(k)}\right)
\end{array}
$$

where

$$
\begin{aligned}
\mathbf{W}(\mathbf{x}) & =\sigma_{n}^{2} \mathbf{D}_{n}(\mathbf{x})^{t} \boldsymbol{\Sigma}_{n}^{-1} \mathbf{D}_{n}(\mathbf{x}) \\
\mathbf{R}(\mathbf{x}) & =\sigma_{x}^{2} \mathbf{D}_{x}(\mathbf{x})^{t} \boldsymbol{\Sigma}_{x}^{-1} \mathbf{D}_{x}(\mathbf{x})
\end{aligned}
$$

\subsection{Simulation}

Four EIT reconstruction types were tested on the proposed algorithm: $\ell_{2}$ norms on both the data residue and the image prior parts $\left(\ell_{2}-\ell_{2}\right) ; \ell_{2}$ norm on the data residue part and 
$\ell_{1}$ norm on image prior $\left(\ell_{2}-\ell_{1}\right) ; \ell_{1}$ on the data residue part and $\ell_{2}$ norm on image prior $\left(\ell_{1}-\ell_{2}\right) ; \ell_{1}$ norm on both parts $\left(\ell_{1}-\ell_{1}\right)$.

Algorithms were implemented for evaluation of 2D EIT problems using the EIDORS software (Adler and Lionheart, 2006). Numerical simulations were conducted using an FEM model with 576 elements. Illustrated as Fig. 6.1: 16 electrodes (marked as green dots) were simulated surrounding the medium, using an adjacent stimulation and measurement pattern. Inside this model, there were two inhomogeneous areas with conductivity 2.0, while the background had conductivity 1.0. The noise performance of the algorithms was tested by adding pseudo random, zero mean Gaussian noise with a fixed random seed. $N S R=1 \%$ where $N S R$ is the ratio of noise to signal power. Images were reconstructed on a 1024 element model which differs from the simulation model to avoid the inverse crime (D. Colton, 1998).

The proposed algorithm was tested with ten iterations. The TV prior was used for all algorithms. Hyperparameters were chosen empirically for the best comprise between image resolution and noise performance. If the $\ell_{1}$ norm was applied on data residue, $\lambda=1.0$, elsewhere, $\lambda=0.01$.

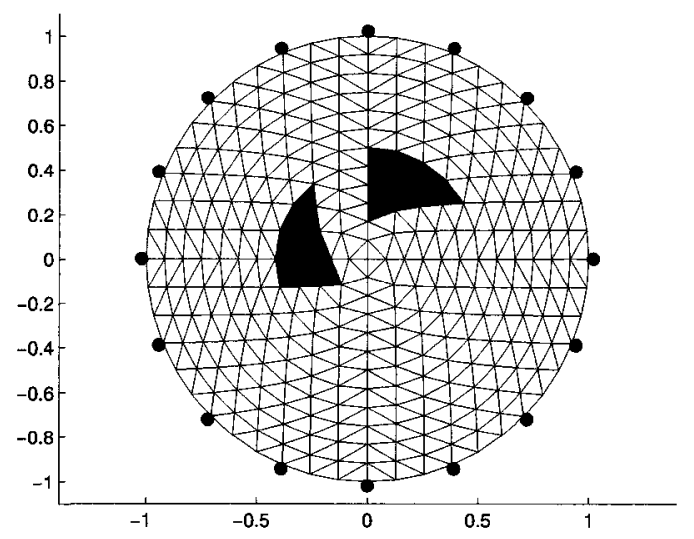

Figure 6.1: Simulation finite element model with 576 elements. Electrodes are indicated by green dots. The background and inhomogeneities have conductivities 1.0 and 2.0, respectively. 


\subsection{Results}

Images were calculated from simulation data using the algorithms discussed in this chapter. Fig. 6.2, compares the reconstructed images from the various choices of $\ell_{1}$ and $\ell_{2}$ prior. (a) is equivalent to the conventional GN method by choosing the $\ell_{2}-\ell_{2}$ norm combination. When applied to the image prior, the $\ell_{1}$ norm obtains better edge sharpness and less artefacts than the $\ell_{2}$ norm.

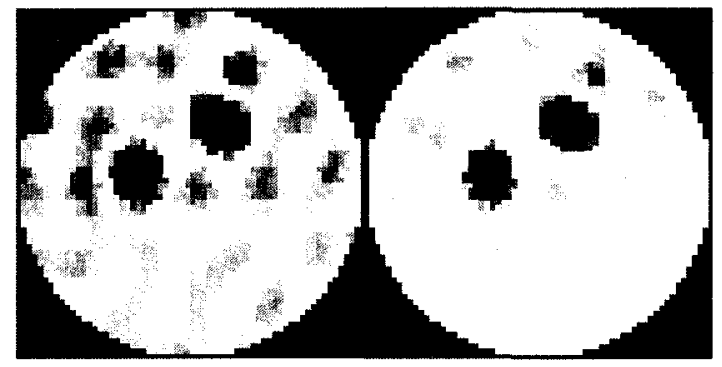

(a)

(b)

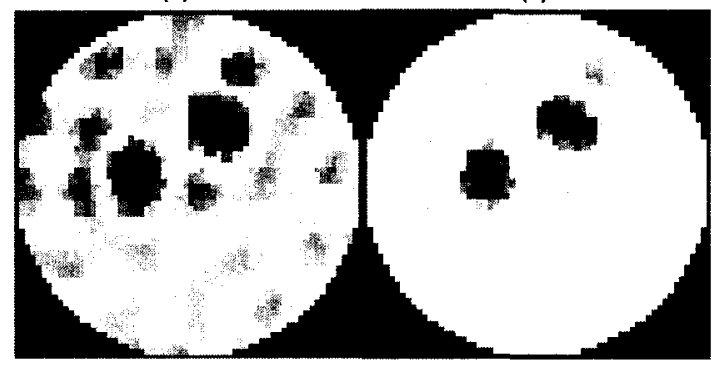

(c)

(d)

Figure 6.2: Images reconstructed using different $\ell_{1}$ and $\ell_{2}$ norms: (a) $p_{n}=2, p_{x}=2\left(\ell_{2}-\ell_{2}\right)$, (b) $p_{n}=2, p_{x}=1\left(\ell_{2}-\ell_{1}\right),(\mathrm{c}) p_{n}=1, p_{x}=2\left(\ell_{1}-\ell_{2}\right),(\mathrm{d}) p_{n}=1, p_{x}=1\left(\ell_{1}-\ell_{1}\right)$.

In order to evaluate the data error robustness of the different norm types, data errors (outliers) were deliberately introduced. Assuming that for certain electrode malfunction, the measurement failure rate was $5 \%$ where electrodes cannot sense voltages. The measurement failure happens randomly. In this simulation, this erroneous effect was implemented by randomly choosing 10 (out of 208) data and set them as zeros. By repeating the same reconstructions as Fig. 6.2, the corresponding "electrode-error" images are generated, and shown in Fig. 6.3. When $\ell_{2}$ norm is used for the data residue term, the reconstructed image shows only noise (Fig. 6.3(a)(b)); however, with the $\ell_{1}$ norm on the data residue (Fig. 6.3(c)(d)) the reconstructed images are very similar to the error free case. This shows high resistance of $\ell_{1}$ solutions against data errors. 

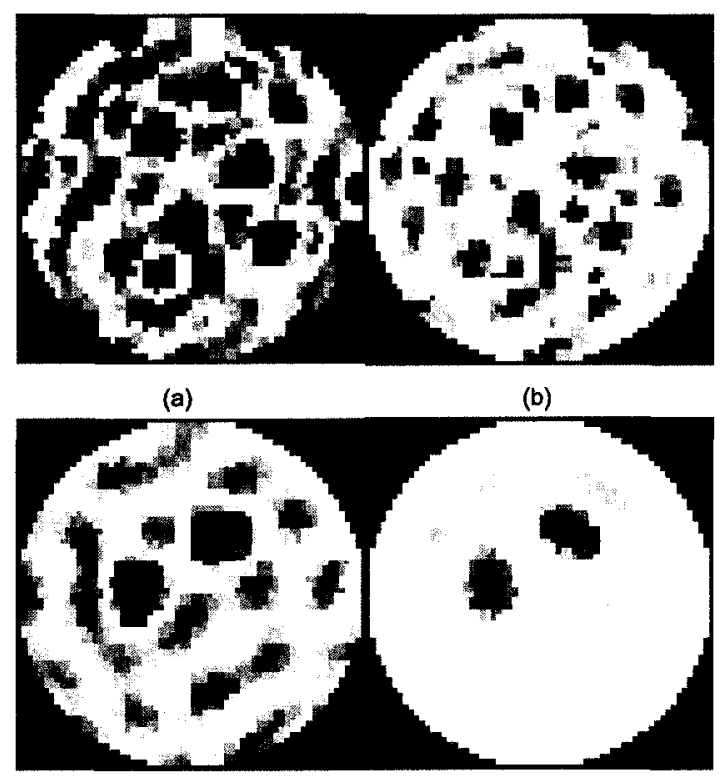

(c)

(d)

Figure 6.3: Electrode error was added to data. Images reconstructed with different data norms: (a) $p_{n}=2, p_{x}=2\left(\ell_{2}-\ell_{2}\right) ;(\mathrm{b}) p_{n}=2, p_{x}=1\left(\ell_{2}-\ell_{1}\right) ;(\mathrm{c}) p_{n}=1, p_{x}=2\left(\ell_{1}-\ell_{2}\right) ;(\mathrm{d}) p_{n}=1, p_{x}=1$ $\left(\ell_{1}-\ell_{1}\right)$

\subsection{Discussion}

EIT images reconstructed using an $\ell_{1}$ norm formulation give two distinct advantages: edge preservation (when $\ell_{1}$ norm is applied to the image priors term), and error robustness (when applied to the data residue term). However, one of advantages of $\ell_{1}$ norm minimization is that it gives a sparse solution which is not accurate when the targets occupies large portion of the image. Another disadvantage is that the $\ell_{1}$ norm formulation cannot be computed as a linear one-step reconstruction due to non-differentiability. Thus, $\ell_{1}$ norm image reconstruction requires an iterative algorithm which is computationally efficient. In this chapter, an efficient iterative method for EIT reconstruction is proposed, which allows, arbitrary choice of data and image prior norms $\left(p_{n}\right.$ and $\left.p_{x}\right)$ to be implemented. Results suggest that $\ell_{1}$ norms on both terms provide the best images in terms of image resolution and robustness to data noise. 


\section{Chapter 7}

\section{In Vivo Blood Characterization from Bioimpedance Spectroscopy of Blood Pooling}

\subsection{Summary}

This chapter is based on the paper-In Vivo Blood Characterization from Bioimpedance Spectroscopy of Blood Pooling accepted by IEEE Transactions on Instrumentation and Measurement in August, 2008. Authors are Tao Dai and Andy Adler.

Characterization of blood impedance properties is important to estimate clinical diagnostic indices such as haematocrit, glucose level and hydration. Current in vivo bioimpedance spectroscopy methods are performed on a body appendage and thus represent a combined measurement of all tissues in the measurement field, rather than the blood individually. This chapter describes a novel in vivo measurement technique to calculate bioelectrical properties of blood while excluding the disturbances from surrounding tissues, based on analysis of the impedance changes caused by blood accumulation. The forearm was modelled as a cylinder containing anatomical structures such as skin-fat layer, muscles, bones. Blood volume was modeled as the inner cylinder. A tetrapolar electrode system was applied to a human forearm and the impedance curves measured with and without blood pooling were processed to calculate the impedance parameters of arterial blood. The bioelectrical parameters of blood were estimated by fitting the blood curve to a Cole-Cole model using the Levenberg-Marquardt (LM) nonlinear curve fitting method. The approach proposed was verified using an experimental phantom, an equivalent circuit model and a preliminary human experiment. Results show that electrical properties of blood and surrounding tis- 
sues can be separated successfully. Of Cole-Cole parameters, the characteristic frequency $f_{c}$ is the most reliable parameter to characterize blood bioelectrical properties. This method may allow simplified measurement of blood characteristic parameters for many biomedical and clinical monitoring applications.

\subsection{Introduction}

The bioimpedance measurements on humans have seen significant interest because of several advantages, such as low cost, ease of application, non-invasiveness and capability for on-line monitoring (Grimnes and Martinsen, 2000; Valentinuzzi, 1996). The original bioimpedance technique was bioelectrical impedance analysis (BIA). Within a decade, this technique evolved into the more advanced technique known as bioelectrical impedance spectroscopy (BIS), also called multiple-frequency bioimpedance analysis (MFBIA). BIS applies multi-frequency stimulations to measure body impedance, and has been used for applications such as: body fluid measurement (Siconolfi et al., 1997; Thomas et al., 1992) which estimates extracellular fluid (ECF), intracellular fluid (ICF) and total body water (TBW); tissue volume change, such as the impedance plethysmography (Nyboer, 1970); and tissue characterization which is mostly based on Cole-Cole model parameters (Cole, 1940): for example, normal and ischemic tissues were differentiated by comparing $R_{0}$ and $f_{c}$ (Casas et al., 1999); plasma resistance, intracellular resistance and cell membrane capacitance of blood were calculated using three measuring frequencies (Zhao et al., 1993). Characterization of blood bioimpedance properties is of importance for the development of methods estimating some clinical diagnostic indices such as haematocrit, glucose level and hydration. However, current bioimpedance spectroscopy measurements of blood are either in vitro (Zhao et al., 1993; Alison and Sheppard, 1993) or are performed on a body appendage and thus represent a combined measurement of all tissues in the measuring field (Brown et al., 1994), rather than the blood impedance value. In this chapter, we propose an in vivo measurement strategy to calculate bioelectrical properties of arterial blood based on the bioimpedance signal from blood pooling in forearm. This work extends our previous conference publication(Dai and Adler, 2006).

Blood pooling methods were adopted to measure the fluid and blood volume change of the abdomen, thigh, and calf of aircraft pilots (Khan and Guha, 2002; Ebert et al., 1986). This method occludes veins to prevent blood flowing out of the segment being investigated.

In this chapter, we propose a novel scheme to measure blood impedance in vivo by analyzing the difference of bioimpedance spectroscopies before and after blood pooling. 
By using this method, blood parameters can be separated from those of the surrounding tissues.

\subsection{Methods}

We consider a tetrapolar impedance sensor applied to the human forearm so that an alternating current enters the forearm from two injection electrodes and the voltage is measured between two measurement electrodes. The physiological structure of this compartment is relatively simple compared with other measuring sites (e.g., chest) and can be approximated with a cylindrical model (Figure 7.1).

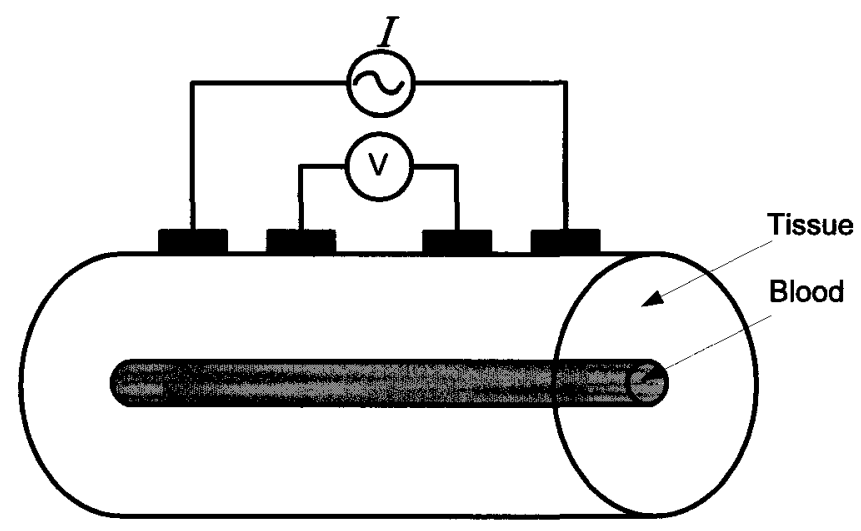

Figure 7.1: A tetrapolar BIS sensor applied to a forearm model. The tetrapolar BIS sensor is composed of a pair of current injectors (outer) and a pair of voltage sensors (inner). The forearm is modelled as a cylinder which is composed of two axial compartments: blood and other tissues. The conductivity distribution is uniform in the axial direction. The former simulate volume of blood; the latter contains tissues in the forearm except for blood, e.g., muscle, fat. The volume of blood increases due to blood pooling.

Blood volume is simulated as an inner cylinder and other tissues are simulated to be in the outer cylinder. Due to blood accumulation, the cross section area of the inner cylinder increases from $S_{a}$ to $S_{a}+\Delta S_{a}$ and the impedance of this segment decreases correspondingly from $Z_{a}$ to $Z_{a}-\Delta Z_{a}$. The fractional variation is thus $\Delta Z_{a} / Z_{a}=\Delta S_{a} / S_{a}$.

There are two states for blood volume: unconstrained: blood volume is minimum, corresponding to impedance value $Z_{s}$; blood accumulated: with an incremental volume on top of the static volume, corresponding to a lower impedance value $Z_{p}$. Based on assumption that blood pooling does not change volume of surrounding tissues, the blood 
pooling model of a forearm can be described as three electrical components in parallel (Figure 7.2).

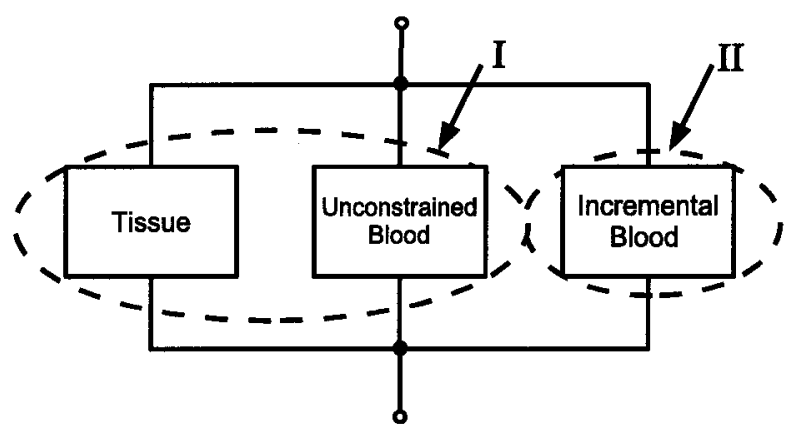

Figure 7.2: In term of electrical structure, the forearm measured is modelled as three components in parallel. Tissue and static blood are grouped as part I (with impedance $Z_{I}$ ), while the incremental blood is part II (with impedance $Z_{I I}$ ). During unconstrained status, the model is represented by part I; during blood pooling, the model is part I paralleled with part II due to infused blood volume.

The impedance measurements, $Z_{s}$ and $Z_{p}$, originate from the tissue model (Figure 7.2) where tissue and static blood are represented by impedance $Z_{I}$, and incremental blood is represented by impedance $Z_{I I}$. During static status, $Z_{s}=Z_{I}$, while after blood accumulation, $Z_{p}=Z_{I} \| Z_{I I}$.

Traditionally, a bioimpedance locus of a tissue can be analyzed using Cole-Cole model. According to Cole (1940), a bioimpedance spectrum can be fitted to the Cole-Cole equation, given by (7.1), (illustrated as Figure 7.3)

$$
Z_{i b}(f)=R_{\infty}+\frac{\Delta R}{1+j\left(f / f_{c}\right)^{1-\alpha}}
$$

where $R_{\infty}$ is the resistance at infinite frequency; $\Delta R=R_{0}-R_{\infty}$, where $R_{0}$ is the resistance at zero frequency; $f_{c}$ is the characteristic frequency of the tissue or model under analysis; and $\alpha$ is the constant depending on the heterogeneity of the tissue, where 0 represents completely homogeneous and 1 completely heterogeneous tissue.

The Cole-Cole curve of the incremental blood (part II, in Figure 7.2) is calculated from two measurements: 1) the impedance spectrum $Z_{s}(f)=Z_{I}$ before blood pooling, and 2) the impedance spectrum $Z_{p}(f)=Z_{I} \| Z_{I I}$ after blood pooling. Based on these data the incremental blood impedance spectrum $Z_{i b}(f)=Z_{I I}$ is calculated by

$$
Z_{i b}(f)=\frac{Z_{s}(f) Z_{p}(f)}{Z_{s}(f)-Z_{p}(f)}
$$




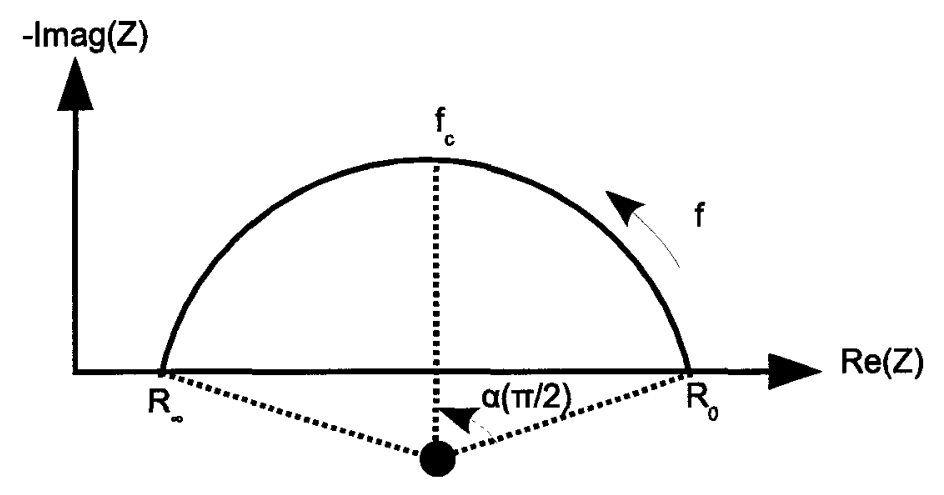

Figure 7.3: Cole-Cole plot in the complex impedance plane

which is derived from the parallel circuit illustrated by Figure 7.2. The calculated $Z_{i b}(f)$ is blood-related only.

Theoretically and without noise, four independent equations are needed to calculate four Cole-Cole parameters. However, due to the data noise, solving the parameters from the theoretically minimal data set would result in large errors. It is thus necessary to use a method which calculates an approximate solution which fits the all of the measured data. In practice, Cole-Cole approximation is accomplished by curve fitting of experimental data to a semi-circular arc in the complex impedance plane, illustrated as Figure 7.3. In this chapter, data were fitted using the Levenberg-Marquardt (LM) algorithm (Moré, 1977). The Cole-Cole model reduces a complex impedance spectrum to four parameters that can be interpreted as physical properties of the tissue under study. The resulting model parameter vector is $m=\left[R_{0}, \Delta R, f_{c}, \alpha\right]$.

Of the Cole-Cole parameters obtained, $R_{0}$ and $R_{\infty}$ are blood properties, as well as functions of body segment geometry and electrode configuration, e.g., body segment geometric changes, electrode movement, and blood pressure changes; while $f_{c}$ and $\alpha$ are functions of blood properties alone. However, $\alpha$ is known to be sensitive to variability in measurements, and relatively insensitive to variations in tissue properties, while $f_{c}$ is relatively stable with respect to measurement geometry changes and more sensitive to tissue property variations (Casas et al., 1999). This suggests that $f_{c}$ is the most discriminating parameter for characterizing blood bioimpedance properties. $R_{0}, R_{\infty}, \Delta R$ and the ratio $R_{0} / R_{\infty}$ are also helpful if careful calibrations are made. 


\subsection{Methods: experimental}

In order to verify the approach presented, we conducted two experiments: the experimental phantom measurement and the equivalent circuit simulation. Their objectives were to model blood pooling behavior in the forearm and estimate the parameters of interest. A preliminary human experiment is discussed in the Section 7.6.

\subsubsection{Experimental Phantom}

An experimental phantom was built to model the forearm structure and blood accumulation behavior (Figure 7.4). A piece of skinned porcine meat (size: $28 \mathrm{~cm} \times 14 \mathrm{~cm} \times 4 \mathrm{~cm}$ ) was taken from cold storage and placed in room environment $\left(24^{\circ} \mathrm{C}\right.$ and $64 \%$ humidity) for at least six hours to reach a stable temperature in order to eliminate the temperature drift. A groove (length $=25 \mathrm{~cm}$, width $=$ height $=2 \mathrm{~cm}$ ) was cut at the position which was about one third of the meat width. A stick of porcine liver was cut to fill the groove. The meat base is to simulate the unconstrained status of the forearm; the porcine liver is filled to the groove to simulate infused blood after pooling.

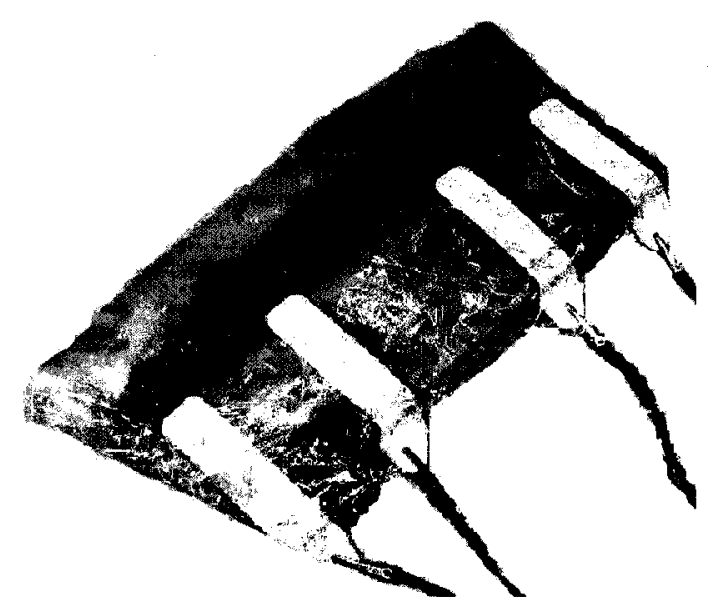

Figure 7.4: Tissue phantom with a tetrapolar BIS sensor applied on the meat surface (background removed from this figure). The phantom was made of a porcine meat base, with a groove cut in it. A filling of porcine liver was filled in the groove. The meat base is to simulate the unconstrained status of the forearm; the porcine liver is filled in the groove to simulate infused blood after pooling.

The goal of this experiment was to validate the approach developed in this chapter. 
From two sets of BIS data of the phantom (measured on meat base with and without liver filling), we estimate the bioelectrical properties of the liver. This corresponds to the model scenario: from two sets of BIS data measured on the forearm (with and without blood accumulation), we calculate the bioelectrical properties of blood.

Four skin electrodes were placed equidistantly onto the surface of the meat, illustrated as Figure 7.4. The inner pair were voltage sensors and the outer pair were current injectors. BIS data were collected from $5 \mathrm{kHz}$ to $1 \mathrm{MHz}$ (at 50 frequencies, logarithmically distributed) by an impedance analyzer (Xitron 4200 ECF-ICF Bioimpedance Analyzer, Xitron Technologies, San Diego, CA, USA).

Firstly, BIS data were measured from the isolated liver filling and Cole-Cole parameters were calculated as the standard to be compared with those of estimated. BIS data were then measured from the meat base with and without liver filling. Cole-Cole parameters were estimated from the measured data using the proposed method. Finally, estimated parameters were compared to the standard parameters obtained initially.

Three pieces of phantom base were tested and each had three liver sticks as fillings. Totally nine experiments were conducted.

\subsubsection{Equivalent Circuit Modeling and Simulation}

A equivalent circuit model was built using electronic components (resistors, capacitors and inductors). This circuit was designed to simulate a biomaterial structure with a cylindrical model (Figure 7.1) based on the following geometric configuration: this model was $5 \mathrm{~cm}$ long with a cross-section area $1 \mathrm{~cm}^{2}$; it was composed of muscle (49\%), fat (49\%) and blood $(2 \%)$, where numbers in parentheses were fractions of the cross-section area (other tissues were ignored for simplicity); blood volume increased by $10 \%$ after blood accumulation.

The dielectric properties of tissues are $\varepsilon_{r}(\omega)$ and $\sigma(\omega)$, where $\varepsilon_{r}$ and $\sigma$ are relative permittivity and conductivity, respectively; $\omega$ is the angular frequency. $\varepsilon_{r}(\omega)$ and $\sigma(\omega)$ were obtained from these reported values: 1) blood (rabbit, in vitro, Gabriel et al. (1996a)); 2) muscle (bovine, paravertebral cut along muscle fibres, in vitro, Gabriel et al. (1996b)); 3) fat (human, in vitro, Gabriel et al. (1996b)). All dielectric data were sampled from the figures in the cited papers and interpolated along 44 points logarithmically distributed from $5 \mathrm{kHz}$ to $10 \mathrm{MHz}$.

The electrical equivalent circuit of certain tissue can be represented by its conductive and capacitive components in parallel (Ivorra, 2002). Each tissue is modelled as a RC 
parallel pair.

$$
Y=S+j \omega C
$$

where $Y$ is the admittance, $S$ is the equivalent conductance and $C$ is the equivalent capacitance. Values of components were calculated by model geometric and dielectric properties described above, using $C=K \varepsilon_{0} \varepsilon_{r}(\omega)$ and $S=K \sigma(\omega) . K=A / l$ is the geometric scale where $A$ is the tissue cross-section area and $l$ is the length. At $5 \mathrm{kHz}$, the equivalent circuit of the biomaterial compartment and corresponding components values are illustrated in Figure 7.5.

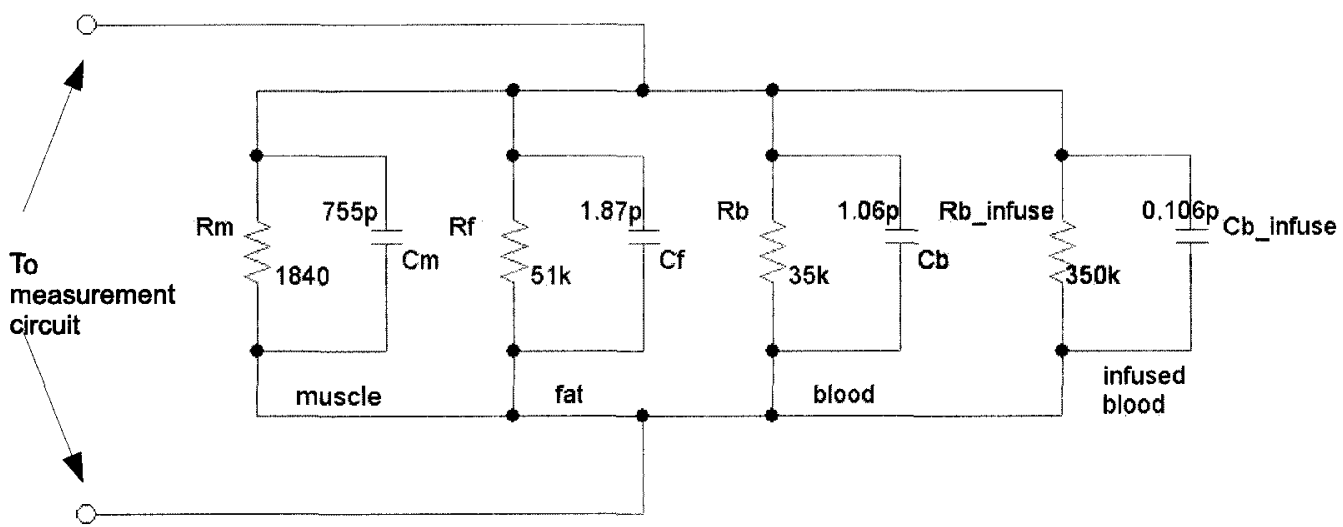

Figure 7.5: units: resistors $-\Omega$; capacitors $-F$. The equivalent circuit of a cylindric biomaterial compartment is composed of muscle, fat, blood and incremental blood. Each element is approximated as an RC parallel pair. Component values were calculated from material composition, geometrical configuration and tissue dielectric properties.

\subsection{Results}

This section presents the results of two experiments described in section 7.4. Estimated parameters were compared with standard values, and the estimation accuracy was investigated.

\subsubsection{Experimental Phantom}

Three pieces of skinned porcine meat (denoted as $1,2,3$ ) were tested as phantom bases and each had three liver sticks as fillings (denoted as a,b,c). Results were obtained from nine experiments. 
Illustrated in Figure 7.6(a), two BIS measurements (each was the time averaging of ten consecutive sweeps), $Z_{\text {base }}(f)$ with and without liver filling, were made and the estimated liver BIS curve was illustrated in Figure 7.6(c), marked as *. By fitting this curve using a $1^{\text {st }}$ order Cole-Cole model (Figure 7.6(c), solid line), the liver's Cole-Cole parameters were calculated and compared with those of the previously measured original liver (Figure 7.6(b), time averaging of ten consecutive sweeps). After being normalized, the original and the estimated liver Cole-Cole curves were compared in Figure 7.6(d). Normalization was performed by dividing real and imaginary parts of impedance by the maxima of real and imaginary values, respectively. The error between the original Cole-Cole model parameters $m_{0}=\left[R_{0}, \Delta R, f_{c}, \alpha\right]$ and the estimated parameters $\hat{m}=\left[\hat{R}_{0}, \Delta R, \hat{f}_{c}, \hat{\alpha}\right]$, was calculated through $e=\left(\left(\left|\hat{m}-m_{0}\right|\right) \cdot / m_{0}\right) \times 100 \%$.

The inductive effect, illustrated as the sub-ripple at the high frequency end (Figure 7.6(b)7.6(c)), can be observed in many real measurements even on homogenous tissues (Lafargue et al., 2002). In our measurements, this effect was also observed above $400 \mathrm{kHz}$. Although this additional effect could still be fitted well by using the ECFC (Extended Cole-Fricke-Cole) model (Lafargue et al., 2002), we chose to fit 40 frequencies (5 kHz to $339 \mathrm{kHz}$ ) to avoid fitting the high frequency subripple, without affecting simulation results. As listed in Table 7.1, The average parameter estimation errors $\left(\mu_{e}\right)$ of $\left[R_{0}, \Delta R, f_{c}, \alpha\right]$ are $[22.35 \%, 15.85 \%, 5.99 \%, 22.79 \%]$, respectively. Compared with other parameters, $f_{c}$ has a much lower error level and a much narrower standard deviation.

\subsubsection{Equivalent Circuit Modeling and Simulation}

According to measurements made (Gabriel et al., 1996a), $f_{c}$ of blood (rabbits, in vitro, room temperature) was about $4.132 \mathrm{MHz}$ which was approximated as the frequency where the value $f \times \varepsilon_{r}$ was the maximum. White Gaussian noise was added into simulation data to test noise performance of the method. We defined $n s r$ as the ratio of noise standard deviation to the amplitude of difference impedance signal. Without noise interference $(n s r=0)$, the Cole-Cole curve of blood was calculated and then fitted by a $1^{s t}$ order ColeCole model, the fitted $\hat{f}_{c}$ was $4.2546 \mathrm{MHz}$ with $2.97 \%$ error compared with the standard $f_{c}(4.132 \mathrm{MHz})$, illustrated by Figure 7.7.

As noise increased, the simulated data no longer showed a consistent pattern and made curve-fitting inaccurate. Figure 7.8 illustrated a successful curve-fitting when $n s r=0.05$. However, when the $n s r$ was increased further, the nonlinear curve fitting could not converge to a reasonable solution. This is determined in the nonlinear curve fitting process by an 


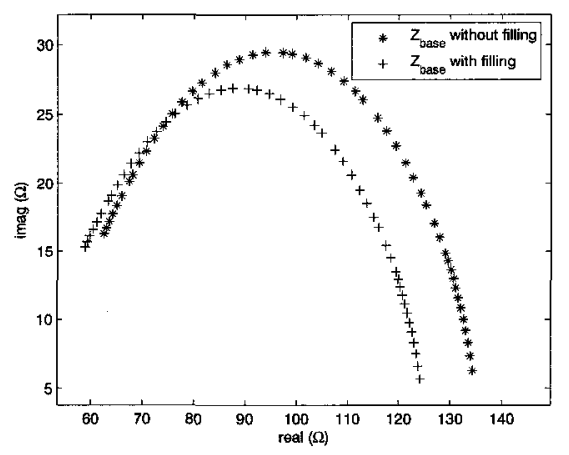

(a)

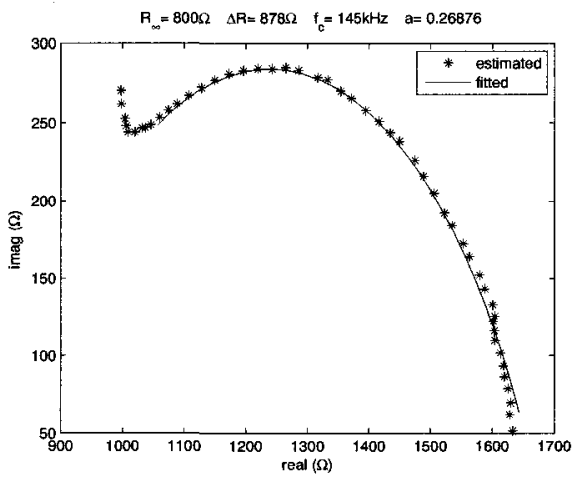

(c)

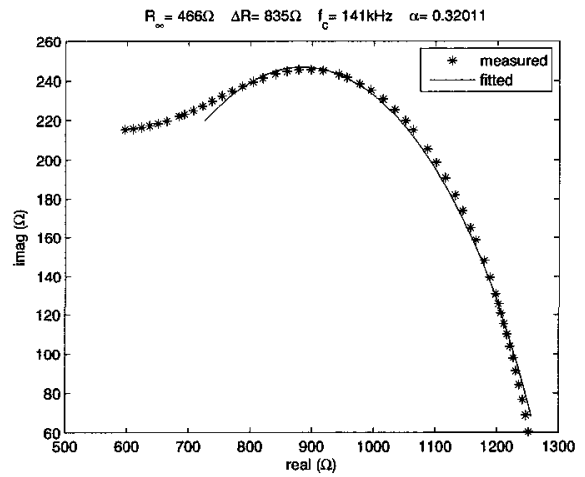

(b)

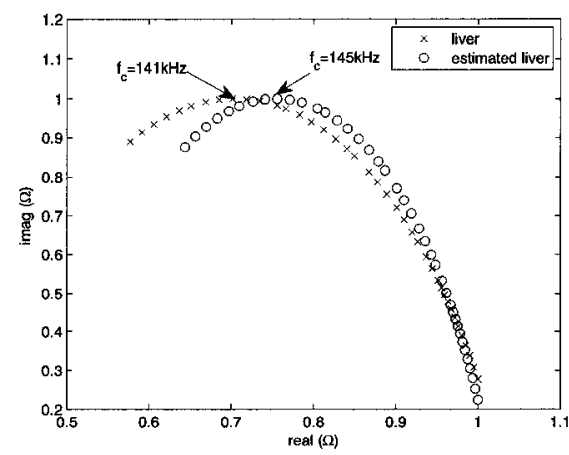

(d)

Figure 7.6: A representative phantom measurement. (a) Cole-Cole curves of the meat base $Z_{\text {base }}(f)$ without $\left({ }^{\prime} *^{\prime}\right)$ and with $\left({ }^{\prime}+{ }^{\prime}\right)$ liver filling; (b) The original Cole-Cole curve of porcine liver stick, $Z_{\text {liver }}(f)$, was fitted in a $1^{\text {st }}$ order Cole-Cole model; The measurement curve and the fitted curve were represented by ${ }^{\prime} *{ }^{\prime}$ and solid line, respectively. (c) From data obtained in (a), the estimated Cole-Cole curve of porcine liver $\hat{Z}_{\text {liver }}(f)$ was calculated using the proposed method. It was fitted in a $1^{\text {st }}$ order Cole-Cole model. The measurement curve and the fitted curve were represented by ' $*$ ' and solid line, respectively. (d) The fitted $Z_{\text {liver }}(f)\left({ }^{\prime} x^{\prime}\right)$ and $\hat{Z}_{\text {liver }}(f)\left({ }^{\prime} o^{\prime}\right)$ were normalized.

extraordinarily large cost function value even after sufficient regression steps.

One practical approach to reduce noise is time averaging where $n s r$ will be decreased by a factor of $\sqrt{N}$ for independent noise, where $N$ is the number of data sets being averaged. Figure 7.9 showed the normalized estimation error $\left(\frac{\hat{f}_{c}-f_{c}}{f_{c}} \times 100 \%\right)$ as a function of $n s r$ and $N$, where 100 independent trials were conducted and means/standard deviations were plotted in the error bar form. For low $n s r$, the estimation errors were below $10 \%$, but jump dramatically when the noise exceeded a certain level, as illustrated in Figure 7.9. 
Table 7.1: Estimated ( $\hat{m})$ v.s Original $\left(m_{0}\right)$ Cole-Cole Parameters:

Measurements were made on three phantom bases (denoted as 1,2,3) and each of which had three liver sticks as fillings (denoted as a,b,c). With the smallest estimation error mean $\mu_{e}$ and error standard deviation $\delta_{e}, f_{c}$ is a good candidate to characterize blood electrically.

\begin{tabular}{|c|c|c|c|c|c|c|c|c|c|c|c|c|c|}
\hline & \multicolumn{3}{|c|}{$R_{0}(\Omega)$} & \multicolumn{3}{|c|}{$\Delta R(\Omega)$} & \multicolumn{3}{|c|}{$f_{c}(k H z)$} & \multicolumn{3}{|c|}{$\alpha$} \\
\hline & & $m_{0}$ & $\hat{m}$ & $\operatorname{err}(\%)$ & $m_{0}$ & $\hat{m}$ & $\operatorname{err}(\%)$ & $m_{0}$ & $\hat{m}$ & $\operatorname{err}(\%)$ & $m_{0}$ & $\hat{m}$ & $\operatorname{err}(\%)$ \\
\hline \multirow{3}{*}{1} & a & 1682 & 1530 & 9.04 & 1010 & 1003 & 0.69 & 157 & 141 & 10.2 & 0.304 & 0.261 & 14.0 \\
\hline & b & 2086 & 1512 & 27.5 & 1277 & 1041 & 18.5 & 155 & 162 & 4.52 & 0.319 & 0.261 & 18.2 \\
\hline & c & 1302 & 1678 & 28.9 & 835 & 878 & 5.15 & 140 & 145 & 3.57 & 0.320 & 0.269 & 15.9 \\
\hline \multirow{3}{*}{2} & a & 1557 & 2076 & 33.3 & 964 & 943 & 2.18 & 189 & 157 & 16.9 & 0.301 & 0.251 & 16.7 \\
\hline & $\mathrm{b}$ & 1796 & 1986 & 10.58 & 1185 & 854 & 27.9 & 149 & 133 & 10.7 & 0.329 & 0.233 & 29.2 \\
\hline & $\mathrm{c}$ & 1369 & 1545 & 12.9 & 918 & 886 & 3.49 & 182 & 181 & 0.55 & 0.294 & 0.276 & 6.12 \\
\hline \multirow{3}{*}{3} & a & 1651 & 1273 & 22.9 & 1455 & 770 & 47.1 & 149 & 150 & 0.67 & 0.438 & 0.316 & 27.8 \\
\hline & $\mathrm{b}$ & 1535 & 2028 & 32.1 & 875 & 668 & 23.7 & 193 & 195 & 1.04 & 0.263 & 0.153 & 41.9 \\
\hline & $\mathrm{c}$ & 2088 & 1590 & 23.9 & 1279 & 1100 & 14.00 & 155 & 164 & 5.81 & 0.317 & 0.206 & 35.0 \\
\hline \multicolumn{2}{|c|}{$\mu_{e}(\%)$} & \multicolumn{3}{|r|}{22.35} & \multicolumn{3}{|r|}{15.85} & \multicolumn{3}{|r|}{99} & \multicolumn{3}{|r|}{2.79} \\
\hline \multicolumn{2}{|c|}{$\delta_{e}(\%)$} & \multicolumn{3}{|r|}{9.30} & \multicolumn{3}{|r|}{15.32} & \multicolumn{3}{|r|}{5.58} & \multicolumn{3}{|r|}{11.41} \\
\hline
\end{tabular}

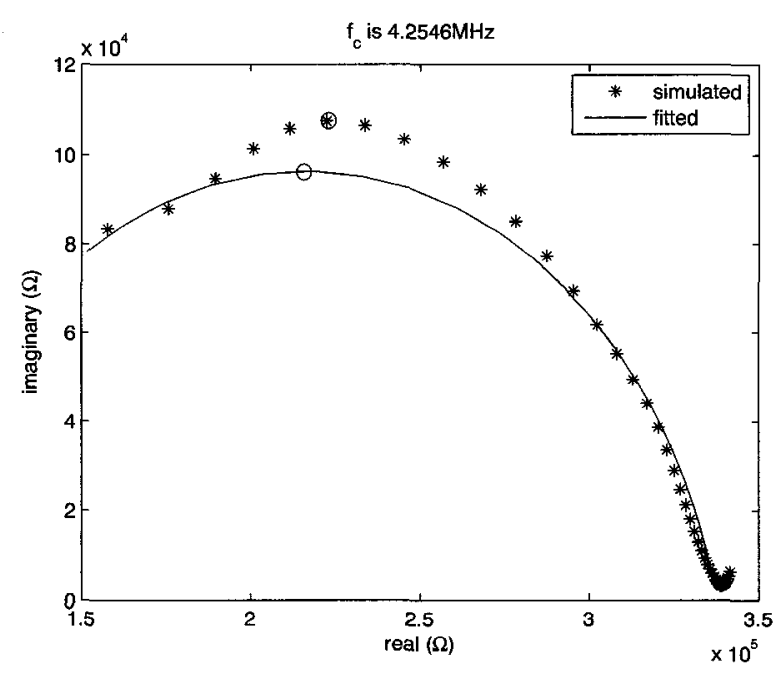

Figure 7.7: Blood impedance curve calculated from circuit simulation (denoted as ${ }^{\prime}{ }^{\prime}$ ) and fitted with a $1^{\text {st }}$ order Cole-Cole model (solid line). Points of $f_{c}$ had been circled out on curves. The estimated $\hat{f}_{c}$ was $4.2546 \mathrm{MHz}$ with $2.97 \%$ deviation to the true $f_{c}$, where $n s r=0$. 


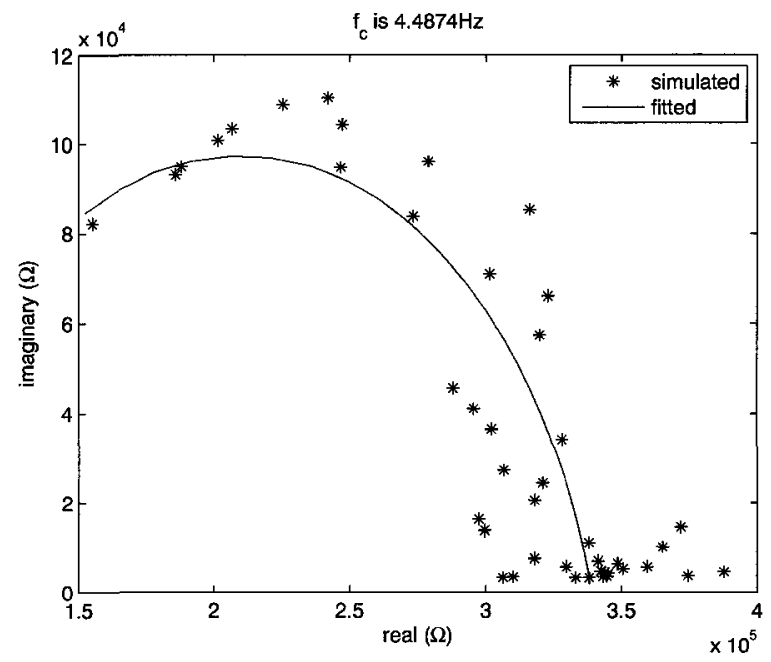

Figure 7.8: Blood impedance curve calculated from circuit model with $n s r=0.05$ (denoted as ' $*$ ') and fitted with a $1^{\text {st }}$ order Cole-Cole model (solid line). The estimated $\hat{f}_{c}$ was $4.4874 \mathrm{MHz}$, $8.60 \%$ deviation to the true $f_{c}$.

\subsection{Discussion}

This chapter introduces a method to measure differential blood impedance curve by processing bioimpedance spectroscopy data before and after blood pooling. This technique offers the advantage that the blood impedance curve is caused by a single medium, blood, while the global impedance signal is composed of all tissues lying in the field of view. Parameters (such as $f_{c}$ ) of this blood curve may be more accurate for blood characterization, compared with those from curves such as $Z_{s}(f)$ or $Z_{p}(f)$ which are generated from multiple tissues due to heterogeneity in the measuring field.

To perform a preliminary in vivo validation of this technique, we conducted a blood pooling experiment on a human subject. A resting, healthy adult was seated with his left forearm resting on the table, the elbow approximately at the level of the heart. A tetrapolar sensor was applied on the forearm (Figure 7.10). The distance between two current injection electrodes was $15 \mathrm{~cm}$ and that of two voltage measurement sensors was $10 \mathrm{~cm}$. BIS data were collected from $50 \mathrm{kHz}$ to $1 \mathrm{MHz}$ using the impedance analyzer (Xitron 4200 ECF-ICF Bioimpedance Analyzer, Xitron Technologies, San Diego, CA, USA). A cuff from a blood pressure meter was applied to the upper arm. The first BIS data set was measured before blood pooling (Figure 7.11(a), ' $*$ ) and then the cuff pressure was increased to $100 \mathrm{mmHg}$ which was between the normal systolic pressure and diastole pressure. This enabled the blood pooling which let arterial blood flow into the forearm but 


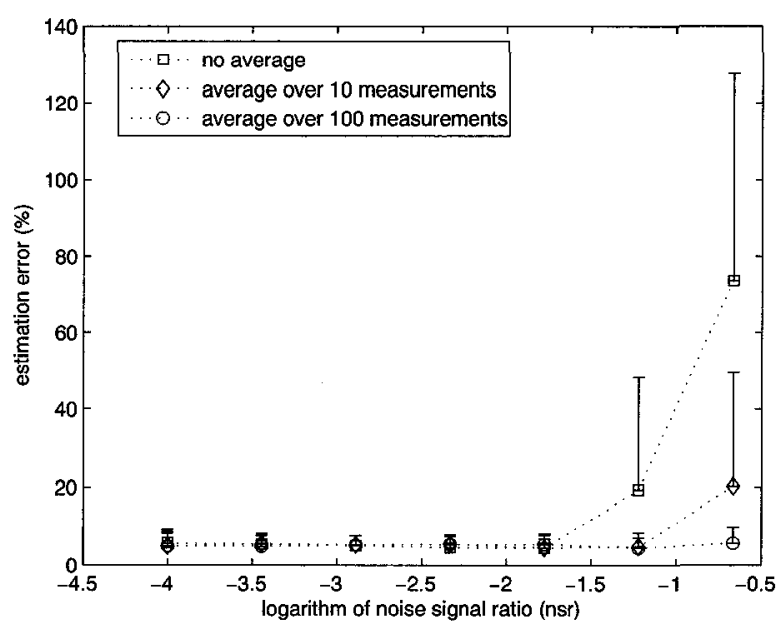

Figure 7.9: The error between the estimated characteristic frequency $\hat{f}_{c}$ and the true $f_{c}$ is highly related to noise levels: above certain noise level, the $\hat{f}_{c}$ is not a good estimate of the true $f_{c}$. However, time averaging applied on measurement data largely increases noise tolerance of the approach. Without averaging $(\square)$ the maximum noise level which could achieve reasonable estimations $(\leq 10 \%)$ was NSR $\leq 0.05$; with $N=10(\diamond)$ and $N=100(0)$, the maximum noise levels increased to NSR $=0.1$ and 0.3 , respectively. As illustrated, the maximum noise level which could achieve reasonable estimations $(\leq 10 \%)$ was about $30 \%$ with time averaging on 100 measurements $(\circ)$, while was only about $5 \%$ for the case without time averaging ( $\square$ ).

prevented venous return. The second BIS measurement was conducted after fifteen seconds of blood pooling (Figure $7.11(\mathrm{a}),{ }^{\prime}+^{\prime}$ ). Blood spectroscopic values were calculated from those data using the method proposed in this chapter (Figure 7.11(b)). There are clear differences between the impedance magnitudes in the human experiment (Figure 7.11) and the equivalent circuit simulation (Figure 7.7). These differences arise from the different geometries: compared with the volunteer's forearm, the simulation is geometrically thinner (inter-electrode distance of $5 \mathrm{~cm}$ with cross-section area of $1 \mathrm{~cm}^{2}$ for the model vs $10 \mathrm{~cm}$ and approximately $100 \mathrm{~cm}^{2}$, respectively, for the arm). Additionally, the dielectric tissue properties and blood/body fraction in the arm are unknown, and differ from the model.

The characteristic frequency of human blood is about $1 \sim 3 \mathrm{MHz}$ (Kanai et al., 1987; Zhao et al., 1993; Gabriel et al., 1996c). The impedance analyzer we used (Xitron 4200 ECF-ICF Bioimpedance Analyzer) can only measure up to $1 \mathrm{MHz}$. However, given that this experiment was a preliminary exploration of the proposed technique's in vivo applicability, we can obtain some useful conclusions. As illustrated in figure 7.11(b), the fitted curve can not show a full arc since the data measured are incomplete. Although we are not 


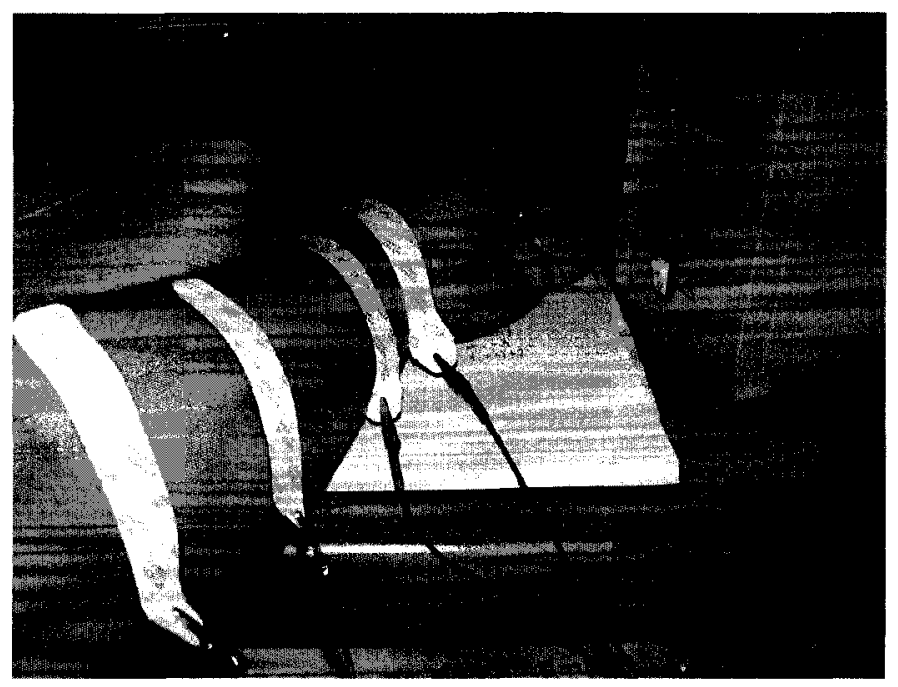

Figure 7.10: A preliminary blood pooling experiment on human arm. A tetrapolar BIS sensor applied on the forearm. Two impedance spectroscopic curves were obtained before and after blood pooling. The upper arm is clamped by a cuff of a blood pressure meter.

able to determine where exactly the $f_{c}$ is, the BIS curve of blood evidently shows that the characteristic frequency is at least $1 \mathrm{MHz}$, because the curve does not reach the highest reactance level which is specified as $f_{c}$ (illustrated as Figure 7.3). This is consistent with previous reports (Kanai et al., 1987; Zhao et al., 1993; Gabriel et al., 1996c).

Some previous work is related to the method we propose. Yamakoshi et al. (1980) showed that the changes in the admittance produced by pulsatile signal in the human finger dipped in the electrolyte vanished when the conductivity of the electrolyte was equal to that of the blood. However, this method was limited by strict experimental conditions. Brown et al. (1994) tried to characterize cardiac related impedance wave measured in the chest and found inappropriate low values of $f_{c}$ while comparing resulting Cole-Cole parameters with those of blood. They demonstrated that the cardiac related impedance wave was not from blood alone but a structure like "blood-tissue" parallel pair and this impedance spectrum could be misleading if used directly for blood characterization. Similar work can be found from Khan and Guha (2002) who carried out blood pooling on a human calf to check bioelectrical variations.

In a blood pooling method such as we propose, the length of the occlusion is a key parameter. Occlusion must be long enough to provide sufficient signal (via the volume of pooled blood). However, too long occlusion results in changes in blood properties via decreased blood $\mathrm{O}_{2}$ saturation, as well as inconvenience to the subject. We selected an 


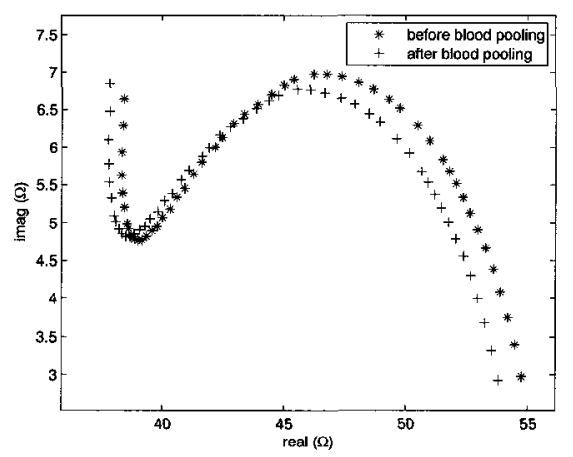

(a)

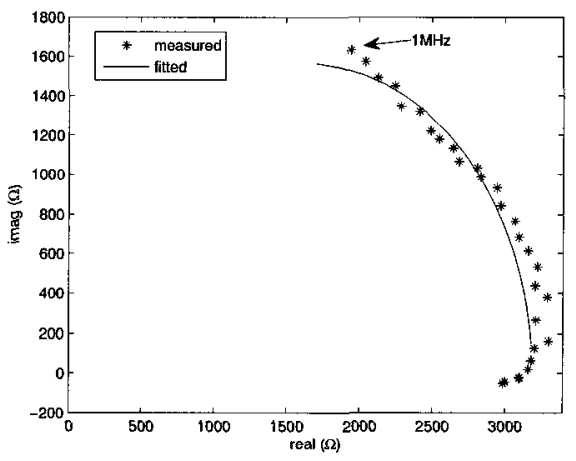

(b)

Figure 7.11: The results of the preliminary human subject experiment. The data were measured from $50 \mathrm{kHz}$ to $1 \mathrm{MHz}$. (a) Cole-Cole curves were measured before $\left({ }^{\prime} *^{\prime}\right)$ and after $\left({ }^{\prime}+{ }^{\prime}\right)$ blood pooling; (b) The Cole-Cole curve of blood, in ' $*^{\prime}$, was calculated from (a) and was fitted in a $1^{\text {st }}$ order Cole-Cole model (solid line). The blood curve is not sufficient to calculate the accurate $f_{c}$ due to measurement device constraint, however, it shows that the characteristic frequency is at least $1 \mathrm{MHz}$.

occlusion time of $15 \mathrm{~s}$ as a compromise. In this chapter, we show that for NSR ratios below $5 \%$, the proposed method is able to calculate reasonably accurate estimates of the blood impedance values. In order to clarify the magnitude of acceptable noise, we first note that bioimpedance measurements are subject to noise from electromagnetic interference, electrode contact impedance, geometrical errors, movement artefacts, and instrument common mode rejection ratio (CMRR). Since this scheme proposes analysis from measurements at the same site close together in time, the electrode contact impedance and geometrical errors may be assumed to be small (since the patient may be assumed to be motionless during measurement). From the results of figure 7.11, measured impedance is approximately $50 \Omega$, and decreases by $3 \Omega$ due to blood pooling. Considering a stimulation current $1 \mathrm{~mA}$, this gives a difference signal of $3 \mathrm{mV}$ with a common mode level of $50 \mathrm{mV}$. Since the electromagnetic interference signal may be reduced by averaging and use of advanced cabling, the noise is limited by the instrument CMRR, which may be considered to be about $60 \mathrm{~dB}$ below $10 \mathrm{MHz}$ for this instrument. In this case, we estimate NSR is $50 \times 10^{-60 / 20} / 3=1.7 \%$ which is well below 5\%. This approximate analysis (and our preliminary human experimental results) suggests that this technique is able to achieve sufficiently low NSR levels to be practically feasible.

In conclusion, we proposed a novel scheme to calculate in vivo properties of blood based on measurements of the blood accumulation induced differential BIS signals. The 
calculated blood curve was fitted using nonlinear curve fitting method and a simple first order Cole-Cole model. Results show that $f_{c}$ is a reliable parameter to characterize blood bioelectrical property in vivo. This method may potentially allow simplified in vivo measurement of blood parameters for many biomedical monitoring applications and clinical diagnoses. 


\section{Chapter 8}

\section{Conclusion and Future Work}

Electrical impedance tomography (EIT) is a non-invasive imaging modality with significant potential clinical applications. Due to merits such as high temporal resolution, low cost, portability. EIT has the potential to be widely applied in medical, industrial and geophysical areas. On the other hand, EIT is severely ill-conditioned and suffers from model errors, such as imperfect body shape meshes, deformation of the subject being measured. In addition, measurement disturbances, such as electrode movement and malfunction, also degrade qualities of reconstructions.

This thesis aims at the improvement of EIT in terms of the image quality and robustness against system errors/measurement noise, by developing advanced regularization frameworks.

\subsection{Conclusions}

The aims of this thesis are achieved as follows:

- Introduction of a novel temporal regularization method.

This method builds a temporal model and directly formulates the temporal inverse in terms of a single regularized expression. This method is illustrated to attain effective lowered artefacts and noise in reconstructions.

- The development of a 4D spatial prior.

On top of the temporal regularization, a novel exponential model considering element spatial correlations is proposed based on: 1) weighted sensitivity, 2) an exponential 
model of inter-element correlations, and 3) a smoothness constraint for poor sensitivity regions. The spatial prior model counts the spatial variation as a random walk process and calculate the inter-element correlation that is independent of element size. Besides merits of the temporal regularization, enhanced image resolution is also illustrated through numerical simulations.

- The development of temporal regularization on both conductivity change and electrode movement.

Images of the conductivity change and electrode movement are calculated in a single step from sequences of EIT data around the current frame. Besides the internal conductivity distribution, variation of boundary shapes is also treated as highly correlated elements. Thus the influence from boundary element movement is effectively removed.

- The development of a generalized iterative reconstruction using the $\ell_{1}$ norm regularization.

EIT images reconstructed using $\ell_{1}$ norm minimizations give two distinct advantages: edge preservation (when $\ell_{1}$ norm is applied to the image priors term), and error robustness (when applied to the data residue term). An efficient iterative method for EIT reconstruction is proposed, which allows, arbitrary choice of data and image prior norms to be implemented in LS means or $\ell_{1}$ means. Results suggest that $\ell_{1}$ norms on both terms provide the best images in terms of resolution and robustness to data defects.

\subsection{Future work}

During the research period of generating all contents of this thesis, many interesting subjects related to main topics are concerned. Although beyond the scope of this thesis, some ideas have already been preliminarily explored. The following subjects are most significant areas of future work that definitely benefit improving the capabilities and integrity of EIT reconstruction algorithms.

- 4D regularization on multi-frequency EIT

By introducing frequency dimension to data, the multi-frequency EIT is also known as spectroscopic EIT. It is well-known that tissues can be well characterized through 
certain bioelectric model, e.g., Cole-Cole model (One of example is illustrated in this thesis-Chapter 7: "In vivo Blood Characterization from Bioimpedance Spectroscopy of Blood Pooling"). Each image element can be described as certain characterized tissue parameter instead of traditional conductivity change. The advantage is significant:

1. Certain characterized parameters are not sensitive to tissue deformation, but only determined by tissue properties. e.g., the characteristic frequency $f_{c}$, as discussed in the Chapter 7 . Therefore, data are collected without significantly affected by patient gesture change and hence avoid movement artefact.

2. It is well-known that bioimpedance spectroscopy (BIS) is good at early stage cancer detection.

3. The integration of the $4 \mathrm{D}$ regularization and spectroscopic measurement can largely enhance imaging accuracy of EIT.

- All-element movement model

In Chapter 5, we investigated temporal regularization on electrode movement model. As we understand, the electrode movement model (Soleimani et al., 2006a) is a subset of the all-element movement model. When one is investigating a "deformable" medium, actually all elements within this medium, not only the boundary elements, have displacements. A preliminary numerical simulation has been done on modeling movements of all medium elements, as part of reconstruction parameters. Significant imaging enhancements have been observed compared with the partial model.

- Variable step size iterative reconstruction.

In Chapter 6, a nonlinear iterative reconstruction provides an efficient solution to solve $\ell_{1}$ norm regularizations. For iterative method, two primary concerns of evaluating algorithm quality are convergence speed and misadjustment (refer to Appendix: A). Adopting a variable step size is widely used, in order to achieve both fast convergence speed and low misadjustment. This effect is sufficiently illustrated in Appendix: A by investigating the VS-APA algorithm. For simplicity, in Chapter 6, a fixed step size is used. We anticipate prominent algorithm optimization by using a properly designed scheme of a variable step size. 
Appendices 


\section{Appendix A}

\section{Variable Step-Size Affine Projection Algorithm with a Weighted and Regularized Projection Matrix}

EIT reconstruction is a system identification problem. The reconstruction methods include linearized one-step solution and nonlinear iterative solution. This appendix describes one of research works of the author in adaptive regression area for system identification.

\section{A.1 Summary}

This appendix is based on the paper Variable Step-Size Affine Projection Algorithm with a Weighted and Regularized Projection Matrix which is submitted to International Journal of Signal Processing by Tao Dai, Andy Adler and Behnam Shahrrava in January, 2008.

This appendix presents a forgetting factor scheme for variable step-size affine projection algorithms (APA). The proposed scheme uses a forgetting processed input matrix as the projection matrix of pseudo-inverse to estimate system deviation. This method introduces temporal weights into the projection matrix, which is typically a better model of the real error's behavior than homogeneous temporal weights. The regularization overcomes the ill-conditioning introduced by both the forgetting process and the increasing size of the input matrix. This algorithm is tested by independent trials with coloured input signals and various parameter combinations. Results show that the proposed algorithm is superior in terms of convergence rate and misadjustment compared to existing algorithms. As a 
special case, a variable step size NLMS with forgetting factor is also presented in this appendix.

\section{A.2 Introduction}

Adaptive signal processing algorithms have been widely used in numerous applications, such as noise cancelation, system identification and data feature extraction. These algorithms are designed to minimize a performance cost function. The Least Mean Squares (LMS) algorithm (Widrow and Stearns, 1985; Haykin, 2002), based on minimizing Mean Squared Error (MSE), is a common algorithm of this type. The Normalized Least Mean Squares (NLMS) (Nagumo and Noda, 1967) algorithm is one of the most widely used adaptive algorithms because of its computational simplicity and robustness. However, highly correlated/colored input signals can deteriorate the convergence rate of LMS type algorithms (Widrow and Stearns, 1985). Recursive Least Squares (RLS) algorithm (Orfanidis, 1985 ) is known for fast convergence even with colored inputs, however, with cost of higher computational complexity. The Affine Projection Algorithm (APA)was proposed by Ozeki and Umeda (1984) using affine subspace projections. As the generalization of the NMLS, the algorithm applies de-correlation of the input process to speed up the convergence, without evident increase of computations and instability problems met by RLS (Yu and Bouchard, 2001). A Fast Affine Projection (FAP) was proposed by Gay and Tavathia (1995) for acoustic echo cancelation. Shin and Sayed (2003) provided a unified treatment of the transient performance of the APA family. Sankaran and Beex (2000) analyzed convergence behaviors of the APA class.

In conventional LMS, NLMS, and APA algorithms, a fixed step size $\mu$ governs the tradeoff between the convergence rate and the misadjustment. To realize both fast convergence and low steady-state deviation, a variable step (VS) is necessary. Harris et al. (1986) used a feedback coefficient based on the sign of the gradient of the squared error; Mader et al. (2000) proposed an optimum step size for NLMS; Shin et al. (2004) proposed a criterion to measure the adaptation states and developed a variable step-size APA based on this criterion.

In this appendix, a forgetting factor method for the variable step size affine projection algorithm is presented. This approach uses a forgetting factor processed input matrix as the projection matrix of pseudo-inverse to estimate weights deviation. Compared with existing algorithms, it significantly improves convergence performance in terms of speed and misadjustment. However, as the input matrix size increases, especially when forget- 
ting process is introduced, the matrix singularity deteriorates and makes the projected error inaccurate. The regularization method resolves this problem and it gives further improvement over the previous method.

\section{A.3 Methods}

\section{A.3.1 Optimal Variable Step-Size APA}

The Affine Projection Algorithm (APA)(Ozeki and Umeda, 1984) updates the weight vector $\mathbf{w}$ via

$$
\mathbf{w}_{i}=\mathbf{w}_{i-1}+\mu U_{i}^{*}\left(U_{i} U_{i}^{*}\right)^{-1} \mathbf{e}_{i}
$$

where

$U_{i}=\left[\begin{array}{c}\mathbf{x}_{i} \\ \mathbf{x}_{i-1} \\ \cdots \\ \mathbf{x}_{i-K+1}\end{array}\right] \mathbf{d}_{i}=\left[\begin{array}{c}d_{i} \\ d_{i-1} \\ \ldots \\ d_{i-K+1}\end{array}\right] \mathbf{w}_{i}=\left[\begin{array}{c}w_{0, i} \\ w_{i, i} \\ \ldots \\ w_{L-1, i}\end{array}\right]$

The subscript $i$ is the time index corresponding to the $i^{\text {th }}$ sampling instant; $K$ is the APA order or signal window width; $L$ is filter order; $\mathbf{w}_{i} \in \mathbb{R}^{L \times 1}$ is the filter weights vector; $\mathbf{x}_{i} \in \mathbb{R}^{1 \times L}$ is the input vector; $d_{i}$ is the desired signal; $\mu$ is the step size; The error signal is $\mathbf{e}_{i}=\mathrm{d}_{i}-U_{i} \mathbf{w}_{i-1}$; the superscript asterisk denotes complex conjugation.

The system input vector, $\mathbf{x}_{i}$, and the desired scalar output, $d_{i}$, are related by

$$
d_{i}=\mathbf{x}_{i} \mathbf{w}^{\circ}+v_{i}
$$

where $\mathbf{w}^{\circ} \in \mathbb{R}^{L \times 1}$ is an unknown vector to be estimated. $v$ is a zero mean Gaussian noise sequence. $\mathbf{x}$ and $v$ are independent.

Shin et al. (2004) proposed the optimal variable step-size APA (VS-APA) in which (A.1) can be written as

$$
\widetilde{\mathbf{w}}_{i}=\widetilde{\mathbf{w}}_{i-1}-\mu U_{i}^{*}\left(U_{i} U_{i}^{*}\right)^{-1} \mathbf{e}_{i}
$$

where $\widetilde{\mathbf{w}}_{i}=\mathbf{w}^{\circ}-\mathbf{w}_{i}$.

$$
\mathbf{p}_{i} \triangleq U_{i}^{*}\left(U_{i} U_{i}^{*}\right)^{-1} U_{i} \widetilde{\mathbf{w}}_{i-1}
$$

which is the projection of $\widetilde{\mathbf{w}}_{i-1}$ onto $\Re\left(U_{i}^{*}\right)$, the range space of $U_{i}^{*}$. Based on the definition of $\mathbf{p}$,

$$
E\left[\mathbf{p}_{i}\right]=E\left[U_{i}^{*}\left(U_{i} U_{i}^{*}\right)^{-1} \mathbf{e}_{i}\right]
$$


where $E(\cdot)$ is the expectation operator. Shin et al. (2004) selected the optimal adaptive filter as the minimizer of $\left\|\mathbf{p}_{i}\right\|$. For this case,

$$
\mathbf{p}_{i}=U_{i}^{*}\left(U_{i} U_{i}^{*}\right)^{-1} \mathbf{e}_{i}
$$

and can be estimated as follows:

$$
\hat{\mathbf{p}}_{i}=\alpha \hat{\mathbf{p}}_{i-1}+(1-\alpha) \mathbf{p}_{i}
$$

by a smoothing factor $\alpha, 0 \leq \alpha<1$. Then the variable step-size APA becomes

$$
\mathbf{w}_{i}=\mathbf{w}_{i-1}+\mu_{i} U_{i}^{*}\left(U_{i} U_{i}^{*}\right)^{-1} \mathbf{e}_{i}
$$

where

$$
\mu_{i}=\mu_{\max } \frac{\left\|\hat{\mathbf{p}}_{i}\right\|^{2}}{\left\|\hat{\mathbf{p}}_{i}\right\|^{2}+C}
$$

and $\|\cdot\|$ denotes the Euclidean norm of a vector. For a positive constant, $C$ is related to $\sigma_{v}^{2} \operatorname{Tr}\left\{E\left[\left(U_{i} U_{i}^{*}\right)^{-1}\right]\right\}$, which can be approximated as $K / S N R$. Here the $\operatorname{Tr}(\cdot)$ is the trace of a matrix. When $\left\|\hat{\mathbf{p}}_{i}\right\|^{2}$ is large, $\mathbf{w}_{i}$ is far from $\mathbf{w}^{\circ}$ and $\mu_{i}$ is close to $\mu_{\text {max }}$; when $\left\|\hat{\mathbf{p}}_{i}\right\|^{2}$ is small, $\mathbf{w}_{i}$ approaches $\mathbf{w}^{\circ}$ and $\mu_{i}$ is close to zero.

\section{A.3.2 Optimal Variable Step Size APA with Forgetting Factor}

In this section, a variable step size APA with a forgetting factor $\lambda$ is proposed. The motivation is similar as that of the RLS algorithm. Choosing a value for $\lambda$ that is less than one introduces memory into the the structure of matrix $U_{i}$, because such a weighting scheme would assign relatively larger weights to recent input samples and smaller weights to input samples in the remote past.

One entry of the input matrix $U_{i}$ is generally denoted as $x_{i-k-l}$ that is in the $(k+1)^{t h}$ row and $(l+1)^{t h}$ column of $U_{i}$ for $k=0, \ldots, K-1$ and $l=0, \ldots, L-1$.

By introducing a forgetting factor $\lambda$, where $0<\lambda \leq 1$, and multiplying every element in $U_{i}$ by $\lambda^{k+l}$, a new observation matrix $Q_{i}$ is introduced where its elements are given by

$$
q_{i-k-l}=\lambda^{k+l} x_{i-k-l},
$$

where $q_{i-k-l}$ denotes the element in row $k+1$ and column $l+1$ of $Q_{i}$ for $k=0, \ldots, K-1$ and $l=0, \ldots, L-1$.

For simplicity, by decomposing the factor $\lambda^{k+l}$ into two terms, (A.10) can be rewritten as follows:

$$
q_{i-k-l}=\lambda^{k} x_{i-k-l} \lambda^{l}
$$


Then (A.11) can be written in matrix form as

$$
Q_{i}=\Lambda^{(K)} U_{i} \Lambda^{(L)}
$$

where $\Lambda^{(K)}=\operatorname{diag}\left(\lambda^{0}, \lambda^{1}, \ldots, \lambda^{K-1}\right)$ and $\Lambda^{(L)}=\operatorname{diag}\left(\lambda^{0}, \lambda^{1}, \ldots, \lambda^{L-1}\right)$. Note that $\Lambda^{(K)}$ can be considered as a column operation on matrix $Q_{i}$ that assigns larger weight to recent regressors. Whereas, $\Lambda^{(L)}$ is for row operation on $Q_{i}$ which assigns larger weights to recent element of each regressor.

In this way, the filter has a tracking mechanism that can track slow statistical variations of the measured data. Since the weighting scheme gives recent data more significance, the algorithm is sensitive to system dynamics (Haykin, 2002).

Two algorithms are preliminarily proposed by replacing $U_{i}$ by $Q_{i}$ in (A.8) and (A.6) as follows:

\section{Algorithm 1:}

$$
\begin{aligned}
\mathbf{w}_{i} & =\mathbf{w}_{i-1}+\mu_{i} Q_{i}^{*}\left(Q_{i} Q_{i}^{*}\right)^{-1} \mathbf{e}_{i} \\
\mu_{i} & =\mu_{\max } \frac{\left\|\hat{\mathbf{h}}_{i}\right\|^{2}}{\left\|\hat{\mathbf{h}}_{i}\right\|^{2}+C} \\
\hat{\mathbf{h}}_{i} & =\alpha \hat{\mathbf{h}}_{i-1}+(1-\alpha) \mathbf{h}_{i} \quad 0 \leq \alpha<1
\end{aligned}
$$

\section{Algorithm 2:}

$$
\begin{aligned}
\mathbf{w}_{i} & =\mathbf{w}_{i-1}+\mu_{i} U_{i}^{*}\left(U_{i} U_{i}^{*}\right)^{-1} \mathbf{e}_{i} \\
\mu_{i} & =\mu_{\max } \frac{\left\|\hat{\mathbf{h}}_{i}\right\|^{2}}{\left\|\hat{\mathbf{h}}_{i}\right\|^{2}+C} \\
\hat{\mathbf{h}}_{i} & =\alpha \hat{\mathbf{h}}_{i-1}+(1-\alpha) \mathbf{h}_{i} \quad 0 \leq \alpha<1
\end{aligned}
$$

where in both algorithms

$$
\mathbf{h}_{i} \triangleq Q_{i}^{*}\left(Q_{i} Q_{i}^{*}\right)^{-1} \mathbf{e}_{i}
$$

As seen, the only difference between these two algorithms is that, in the Algorithm $2, U_{i}$ is only replaced by $Q_{i}$ during the error evaluation phase (A.6), not during the weights updating phase (A.14a). The Algorithm 1 is not recommended because: replacing $U_{i}$ by $Q_{i}$ during the both phases introduces possible convergence instability. This is discussed in details in the section A.4.

The algorithm 2 is the proposed variable step size affine projection algorithm with a forgetting factor (VS-APA-FF). A special case is the variable step size NLMS with 
forgetting factor (VS-NLMS-FF). This can be obtained by setting $K=1$ when the input matrix $U_{i}$ is a row vector and the forgetting factor processing is implemented only in the row direction as follows:

$$
Q_{i}=U_{i} \Lambda^{(L)}
$$

\section{A.4 Algorithm Stability Analysis}

The convergence behavior of the general form of APA class had been investigated by Sankaran and Beex (2000). Ikeda (2002) derived the convergence rates of the APA family for both white and the coloured signals. As proofed in (Sankaran and Beex, 2000), $\mu \in$ $(0,2)$ is a necessary and sufficient condition for the APA class to be stable; when $\mu=1$, the algorithm conducts the fastest convergence. Therefore, the proposed variable step size APA with forgetting factor (VS-APA-FF), (Algorithm 2), guarantees stability as long as the initial step size is within $(0,2)$ (Sankaran and Beex, 2000).

To investigate the effect of introducing the forgetting scheme on the weight update, the convergence behavior of the traditional fixed step size APA (Haykin and B.Widrow, 2003) is firstly investigated:

$$
\mathbf{w}_{i}=\mathbf{w}_{i-1}+\mu U_{i}^{*}\left(U_{i} U_{i}^{*}\right)^{-1} \mathbf{e}_{i}
$$

Subtracting both sides of (A.17) from $\mathbf{w}^{\circ}$ leads to the following weight-error recursion:

$$
\widetilde{\mathbf{w}}_{i}=\left[I-\mu U_{i}^{*}\left(U_{i} U_{i}^{*}\right)^{-1} U_{i}\right] \widetilde{\mathbf{w}}_{i-1}
$$

where $I$ is an $L \times L$ identity matrix.

The singular value decomposition (SVD) of the input matrix $U$ is given by

$$
U=R \Sigma V^{*}
$$

where $R$ and $V$ are $K \times K$ and $L \times L$ unitary matrices respectively, and the $K \times L$ matrix $\Sigma$ is defined as

$$
\Sigma=\left[\begin{array}{ll}
\mathbf{S} & 0
\end{array}\right]
$$

where $\mathbf{S}$ is a diagonal matrix,

$$
\mathbf{S}=\operatorname{diag}\left(\sigma_{1}, \sigma_{2}, \ldots, \sigma_{K}\right)
$$

and the $\sigma$ 's which are called the eigenvalues of $U$ are positive square roots of the eigenvalues of $U_{i} U_{i}^{*}$ and are usually ordered in a decreasing manner, $\sigma_{1} \geq \sigma_{2} \geq \ldots \geq \sigma_{K}>0$. 
Substituting (A.19) into (A.18) gives

$$
\widetilde{\mathbf{w}}_{i}=V_{i}\left[I-\mu \Sigma_{i}^{*}\left(\Sigma_{i} \Sigma_{i}^{*}\right)^{-1} \Sigma_{i}\right] V_{i}^{*} \widetilde{\mathbf{w}}_{i-1} .
$$

Multiplying on the left by $V_{i}^{*}$ then gives

$$
V_{i}^{*} \widetilde{\mathbf{w}}_{i}=\left[I-\mu \Sigma_{i}^{*}\left(\Sigma_{i} \Sigma_{i}^{*}\right)^{-1} \Sigma_{i}\right] V_{i}^{*} \widetilde{\mathbf{w}}_{i-1} .
$$

In this result, let define the rotational coefficient error vector as $\breve{\mathbf{w}}_{i}=V_{i}^{*} \widetilde{\mathbf{w}}_{i}$ and write

$$
\breve{\mathbf{w}}_{i}=\left[I-\mu \Sigma_{i}^{*}\left(\Sigma_{i} \Sigma_{i}^{*}\right)^{-1} \Sigma_{i}\right] \breve{\mathbf{w}}_{i-1}
$$

Now each element of $\breve{\mathbf{w}}_{i}$ converges at its own rate, which is determined by the corresponding eigenvalue of the transformation matrix $T_{i}=\left[I-\mu \Sigma_{i}^{*}\left(\Sigma_{i} \Sigma_{i}^{*}\right)^{-1} \Sigma_{i}\right]$. Suppose the $k^{\text {th }}$ eigenvalue of $T_{i}$ is $\rho_{k}, k=1, \ldots, K$, then the $k^{\text {th }}$ element of $\breve{\mathbf{w}}_{i}$ has converge rate as

$$
\rho_{k}=1-\mu \frac{\sigma_{k} \sigma_{k}}{\sigma_{k} \sigma_{k}}=1-\mu
$$

Conclusions: 1. $\mu \in(0,2)$ is the sufficient and necessary condition for $A P A$ class algorithm convergence; 2 . if $\mu=1$, the algorithm converges at the highest rate. These are consistent with the conclusions drawn by Sankaran and Beex (2000).

Next, let investigate the convergence behavior of the APA algorithm if a forgetting weighted process is introduced into the weight updating phase (A.17). After being weighted, the singular value decomposition of the input matrix $Q$ is (here the time index is ignored for simplicity, without loss of generality):

$$
\begin{aligned}
Q & =\Lambda^{(K)} U \Lambda^{(L)}=\Lambda^{(K)}\left[R \Sigma V^{T}\right] \Lambda^{(L)} \\
& =R\left[\Lambda^{(K)} \Sigma \Lambda^{(L)}\right] V^{T}=R \Sigma^{\prime} V^{T}
\end{aligned}
$$

where

$$
\Sigma^{\prime}=\Lambda^{(K)} \Sigma \Lambda^{(L)}
$$

$\Sigma^{\prime} \in \Re^{K \times L}$ and

$$
\left[\Sigma^{\prime}\right]_{k, l}=\left\{\begin{array}{l}
\sigma_{k}^{\prime}=\lambda^{2(k-1)} \sigma_{k} \quad k=l \\
0 \quad k \neq l
\end{array}\right.
$$

for $k=1,2, \ldots, K ; l=1,2, \ldots, L$.

The (A.17) becomes

$$
\mathbf{w}_{i}=\mathbf{w}_{i-1}+\mu Q_{i}^{*}\left(Q_{i} Q_{i}^{*}\right)^{-1} \mathbf{e}_{i}
$$


Subtracting both sides of (A.29) from $\mathbf{w}^{\circ}$

$$
\widetilde{\mathbf{w}}_{i}=\left(I-P_{i}^{\prime}\right) \widetilde{\mathbf{w}}_{i-1}
$$

where $P_{i}^{\prime}=\mu Q_{i}^{*}\left(Q_{i} Q_{i}^{*}\right)^{-1} U_{i}$. The equation (A.24) changes to

$$
\breve{\mathbf{w}}_{i}=\left[I-\mu \Sigma_{i}^{*}\left(\Sigma_{i}^{\prime} \Sigma_{i}^{\prime *}\right)^{-1} \Sigma_{i}\right] \breve{\mathbf{w}}_{i-1}
$$

the weighted transformation matrix becomes $T_{i}^{\prime}=\left[I-\mu \Sigma_{i}^{\prime *}\left(\Sigma_{i}^{\prime} \Sigma_{i}^{\prime *}\right)^{-1} \Sigma_{i}\right]$. Suppose the $k^{\text {th }}$ eigenvalue of $T_{i}^{\prime}$ is $\rho_{k}^{\prime}, k=1, \ldots, K$, then the $k^{\text {th }}$ element of $\breve{\mathbf{w}}_{i}$ has converge rate as

$$
\rho_{k}^{\prime}=1-\mu \frac{\sigma_{k}^{\prime} \sigma_{k}}{\sigma_{k}^{\prime} \sigma_{k}^{\prime}}=1-\mu \frac{\sigma_{k}}{\sigma_{k}^{\prime}}=1-\mu \lambda^{2(1-k)}
$$

when $\lambda$ and $k$ are chosen such that $\frac{2}{\mu}<\lambda^{2(1-k)}$, certain converge rate $\left|\rho_{k}^{\prime}\right|>1$ which means the algorithm is unstable.

Conclusion: The APA algorithm may be unstable if the forget-weighting process is introduced into the weight updating phase (Algorithm 1). Although parameters can be chosen carefully to assure $\left|\rho_{k}^{\prime}\right| \leq 1$, applying the proposed forgetting process on the weight updating phase is not recommended.

In the Section A.5, the proposed algorithm VS-APA-FF is further upgraded.

\section{A.5 Regularization of the Weighted Projection}

According to equation A.6 and A.15, the accuracy of the error evaluation depends on the condition number (define by the ratio of the maximum eigenvalue and the minimum eigenvalue) of the projection matrix $(U$ or $Q$ ). For a projection matrix with large condition number, even a small amplitude of noise in the error signal will be amplified which makes the $\mathbf{h}$ or $\mathbf{p}$ relatively noisy. Thus the $V$ S-APA and $V$ S-APA-FF algorithms adopt a smoothing function, in the form of (A.7), to alleviate this problem, however, with the cost loss of error signal fidelity, which sacrifices convergence speed and/or misadjustment.

In the previously proposed algorithm (Algorithm 2, denoted as VS-APA-FF), the weighted projection matrix $Q$ has a larger condition number compared with the nonweighted $U$. This can be illustrated as follows:

The condition number of $U$ is:

$$
\operatorname{cond} U=\sigma_{\max } / \sigma_{\min }=\sigma_{1} / \sigma_{K}
$$


from (A.12), the SVD of the weighted input matrix $Q$ is:

$$
\begin{aligned}
Q & =\Lambda^{(K)} U \Lambda^{(L)}=\Lambda^{(K)}\left[R \Sigma V^{*}\right] \Lambda^{(L)} \\
& =R\left(\Lambda^{(K)} \Sigma \Lambda^{(L)}\right) V^{*} \\
& =R \Sigma^{\prime} V^{*}
\end{aligned}
$$

where $\Sigma^{\prime}$ is a $K \times L$ matrix with all zero entities except $\left[\Sigma^{\prime}\right]_{j, j}=\lambda^{2(j-1)} \sigma_{j}, j=1,2, \ldots, K$. The condition number of $Q$ is:

$$
\operatorname{cond} Q=\sigma_{1} /\left[\lambda^{2(K-1)} \sigma_{K}\right]=\lambda^{2(1-K)} \operatorname{cond} U
$$

which illustrates the increased condition number by a factor of $\lambda^{2(1-K)}$. According to equation (A.15), this means that the estimated $\mathbf{h}$ is more sensitive to the noise in error signal.

In stead of solving this problem using a smoothing function as the VS-APA and $V S-$ APA-FF, this problem is addressed to use a Tikhonov regularization approach, under which (A.15) becomes:

$$
\mathbf{h}_{i}=Q_{i}{ }^{*}\left(Q_{i} Q_{i}{ }^{*}+\delta^{2} I\right)^{-1} \mathbf{e}_{i} .
$$

where $I$ is the identity matrix on the assumption that each element in $\mathbf{h}_{i}$ has identical variance; $\delta$ is a hyperparameter to control the amount of regularization. The modified algorithm becomes (denoted as VS-APA-FF-REGU:

$$
\begin{aligned}
& \mathbf{w}_{i}=\mathbf{w}_{i-1}+\mu_{i} U_{i}^{*}\left(U_{i} U_{i}^{*}\right)^{-1} \mathbf{e}_{i} \\
& \mu_{i}=\mu_{\max } \frac{\left\|\hat{\mathbf{h}}_{i}\right\|^{2}}{\left\|\hat{\mathbf{h}}_{i}\right\|^{2}+C}
\end{aligned}
$$

Note that the smoothing functions (such as (A.7), (A.14c)) is no longer needed (effectively $\alpha=0$ ), since the regularization process accomplishes this function.

\section{A.6 Simulation Results}

The proposed algorithm is validated by simulations of a system identification model (Figure A.1).

Following the test framework of Shin et al. (2004), the system to be simulated is represented by a moving average model with $L$ taps. The adaptive filter has the same number of taps. The goal of the adaptive processing is to estimate system parameters by optimizing the adaptive filter parameters iteratively using the proposed algorithm. 


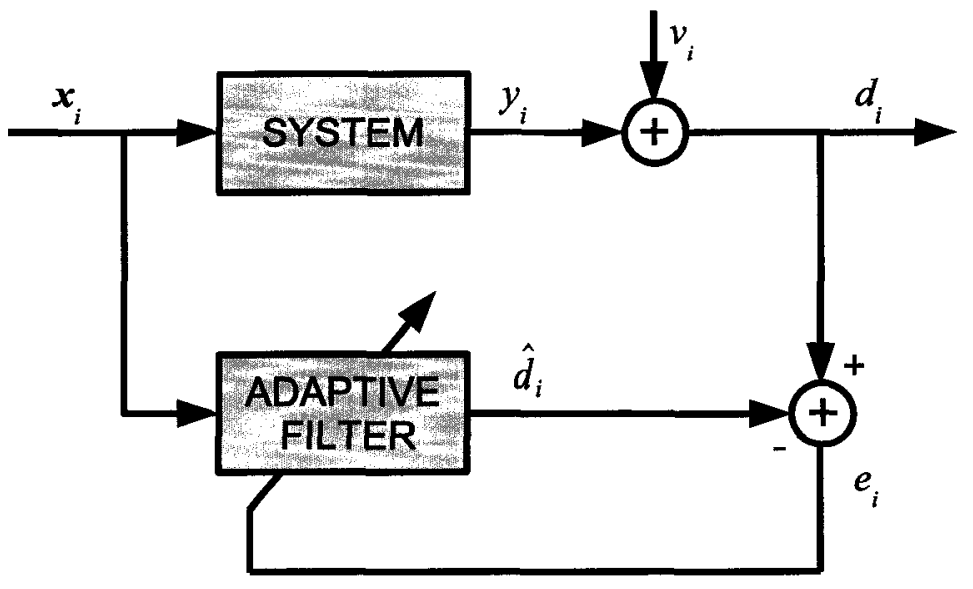

Figure A.1: System identification model

Two coloured Gaussian noises are used as input signals. The input signal colorizations are obtained by filtering a white Gaussian random noise (zero mean, unit variance) through a first order filter, $G_{1}(z)=1 /\left(1-0.9 z^{-1}\right)$ or a $4^{\text {th }}$ order filter

$$
G_{2}(z)=\frac{1+0.9 z^{-1}+0.6 z^{-2}+0.81 z^{-3}-0.329 z^{-4}}{1+z^{-1}+0.21 z^{-2}}
$$

The measurement noise $v_{i}$ is added to $y_{i}\left(y_{i}=\mathbf{x}_{i} \mathbf{w}^{\circ}\right)$ and the SNR of the measurement signal is calculated by

$$
S N R=10 \log _{10}\left(\frac{E\left[y_{i}^{2}\right]}{E\left[v_{i}^{2}\right]}\right)
$$

The simulation results are obtained from the ensemble average of 100 independent trials (ten thousand iterations each), with $\mu_{\max }=1$ and a smoothing factor $\alpha=0.99$ for $V S$ $A P A$ and $V S-A P A-F F$. The convergence is evaluated by Mean Square Deviation (MSD) which is calculated by

$$
E\left(\left\|\tilde{\mathbf{w}}_{i}\right\|^{2}\right)=E\left(\left\|\mathbf{w}^{\circ}-\mathbf{w}_{i}\right\|^{2}\right)
$$

Figures A.2-A.3 illustrate effects of different forgetting factors of VS-APA-FF through G1 and G2 colorizations, respectively. The special case of VS-APA-FF is VS-NLMS-FF (when $\mathrm{K}=1$ ) which is illustrated in Figure A.2(a)A.2(c) and A.3(a). (Note that when $\lambda=1$, the VS-APA-FF becomes the original VS-APA. Therefore, VS-APA can be regarded as a special case of VS-APA-FF).

From figures A.2-A.3, following observations can be made: 


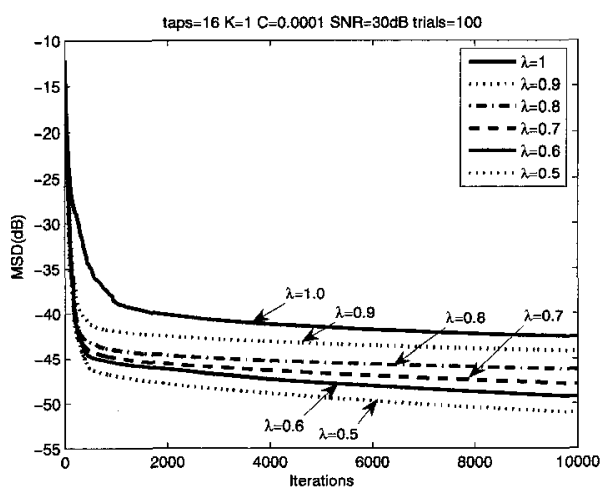

(a)

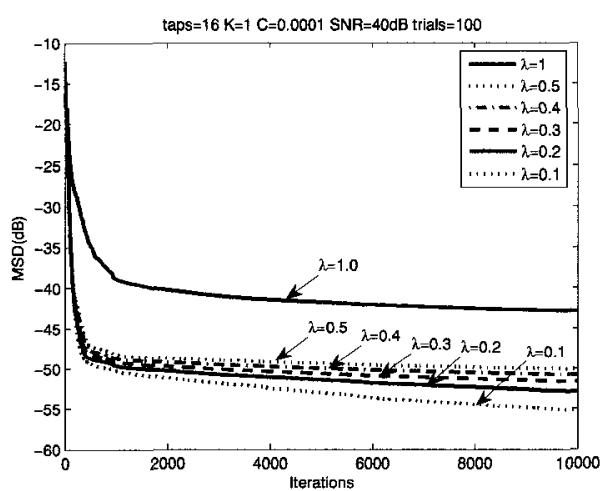

(c)

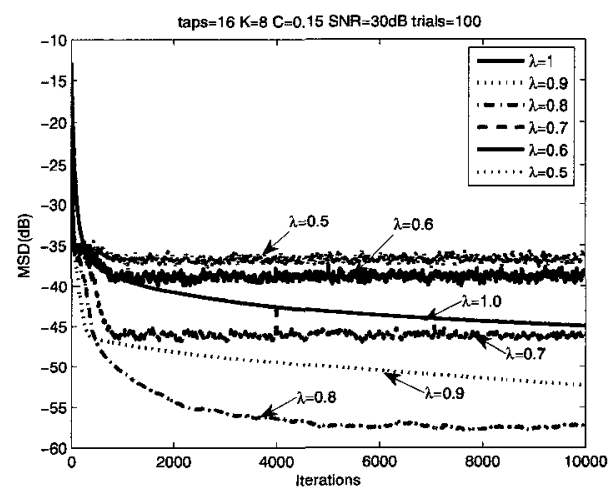

(b)

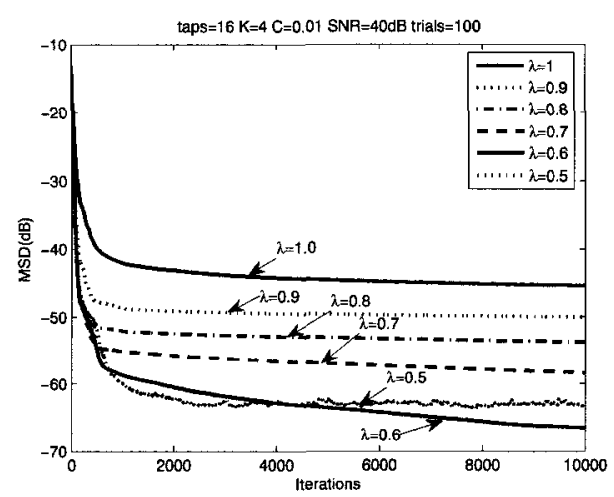

(d)

Figure A.2: Effects of $\lambda$ in VS-APA-FF (VS-APA when $\lambda=1.0$ ), G2 colorization. (a) VSNLMS-FF $\mathrm{K}=1$, taps=16, $\mathrm{C}=0.0001, \mathrm{SNR}=30 \mathrm{~dB}$; (b) $\mathrm{K}=8$, taps=16, $\mathrm{C}=0.15, \mathrm{SNR}=30 \mathrm{~dB}$; (c) $V S-N L M S-F F \mathrm{~K}=1$, taps $=16, \mathrm{C}=0.0001, \mathrm{SNR}=40 \mathrm{~dB}$; d) $\mathrm{K}=4$, taps $=16, \mathrm{C}=0.01, \mathrm{SNR}=40 \mathrm{~dB}$; 


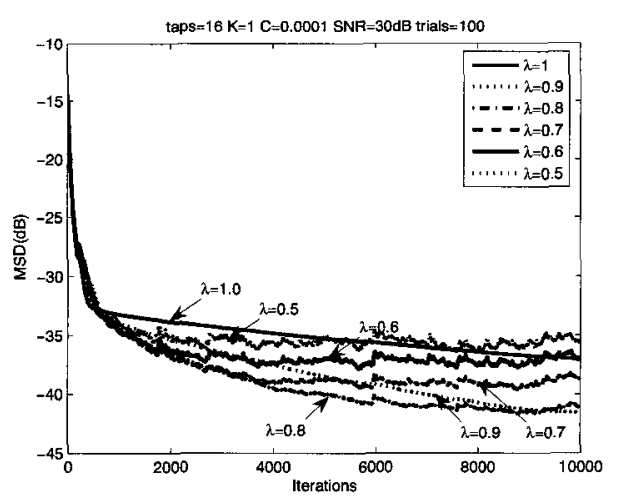

(a)

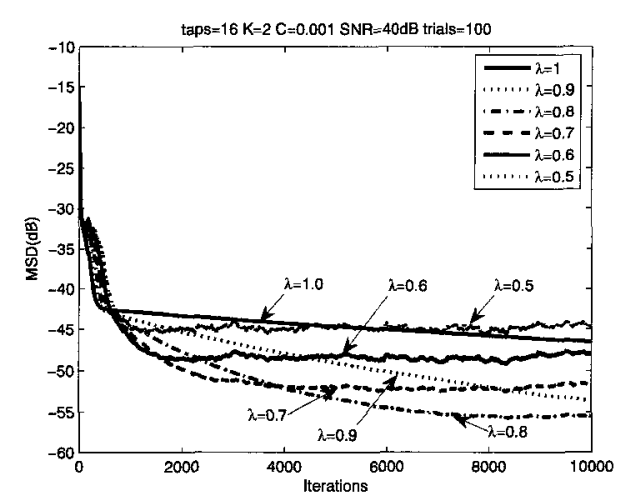

(c)

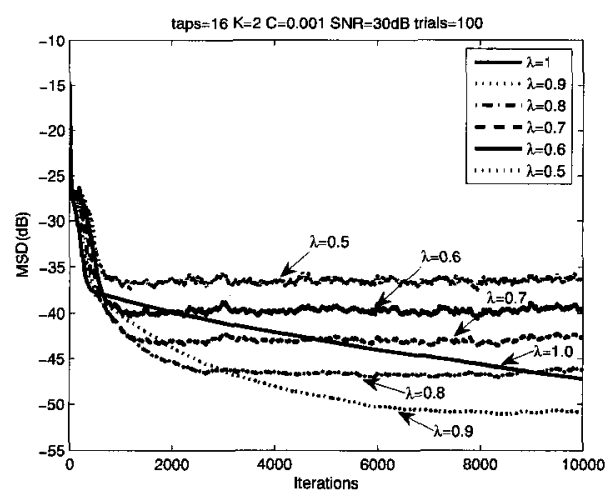

(b)

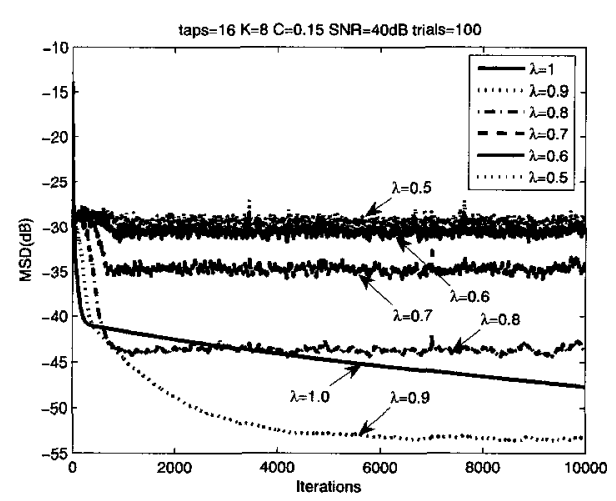

(d)

Figure A.3: Illustration of different forgetting factors for VS-APA-FF(VS-APA when $\lambda=1.0$ ), G1 colorization. (a) $V S-N L M S-F F \mathrm{~K}=1$, taps=16, $\mathrm{C}=0.0001, \mathrm{SNR}=30 \mathrm{~dB}$; (b) $\mathrm{K}=2$, taps $=16$, $\mathrm{C}=0.001, \mathrm{SNR}=30 \mathrm{~dB}$; (c) $\mathrm{K}=2$, taps $=16, \mathrm{C}=0.001, \mathrm{SNR}=40 \mathrm{~dB} ;$ (d) $\mathrm{K}=8$, taps=16, $\mathrm{C}=0.15$, $\mathrm{SNR}=40 \mathrm{~dB}$;

- VS-APA-FF outperforms VS-APA by applying a temporal weighted projection ma$\operatorname{trix} Q$.

- in Figures A.2-A.3, some adaptation curves are noisy (e.g. Figures A.2(b)and Figures A.3(d), when $K$ is large and $\lambda$ is small), which also illustrated the increased condition number of the projection matrix.

- noise color affects adaptation performance. (This applies for all APA class)

Using experimental conditions described previously, and $\lambda=0.5, \delta=1$, simulation comparisons between VS-APA, VS-APA-FF and the regularized version VS-APAFF-REGU are illustrated by figure A.4 (noise G2) and figure A.5 (noise G1). For some 
cases, when the update matrix of VS-APA-FF becomes severly ill-conditioned (Figure A.4(a)A.5(a)A.5(b)A.5(c)) and experiences unsatisfactory convergence, the VS-APA-FFREGU can still converge quickly with low misadjustment. Therefore, the conclusion is that VS-APA-FF-REGU is a good complement for VS-APA-FF, when the forgetting processed input matrix is close to singular.

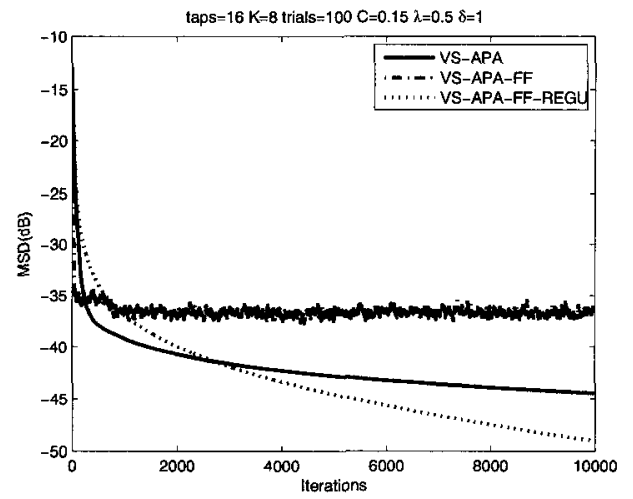

(a)

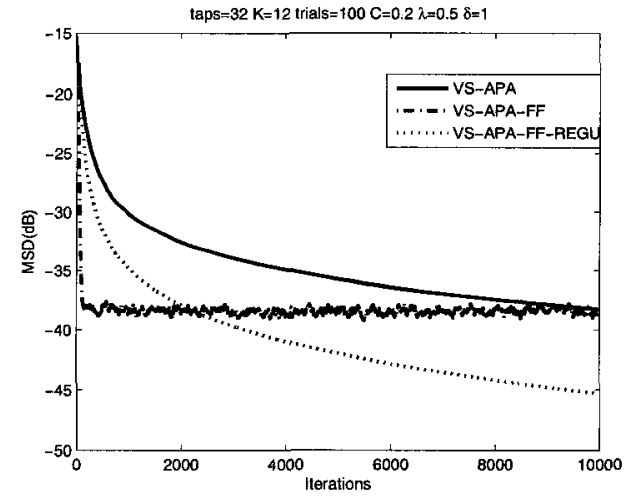

(b)

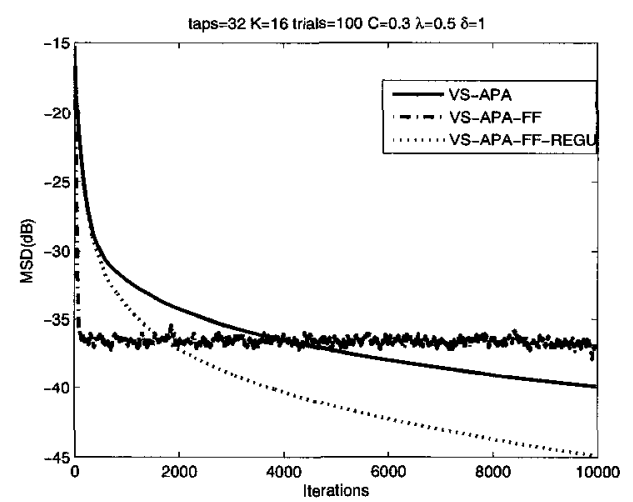

(c)

Figure A.4: Comparisons among VS-APA, VS-APA-FF, and VS-APA-FF-REGU, G2 colorization. $\lambda=0.5$. (a) $\mathrm{K}=8$, taps $=16, \mathrm{C}=0.15$; (b) $\mathrm{K}=12$, taps $=32, \mathrm{C}=0.2 ;$ (c) $\mathrm{K}=16$, taps $=32, \mathrm{C}=0.3$

On the other hand, by using a larger forgetting factor $(e . g ., \lambda=0.9)$ when the VSAPA-FF has less singularity problem, the VS-APA-FF-REGU is still advantageous over the VS-APA and the VS-APA-FF, illustrated in Figures (A.6,A.7). 


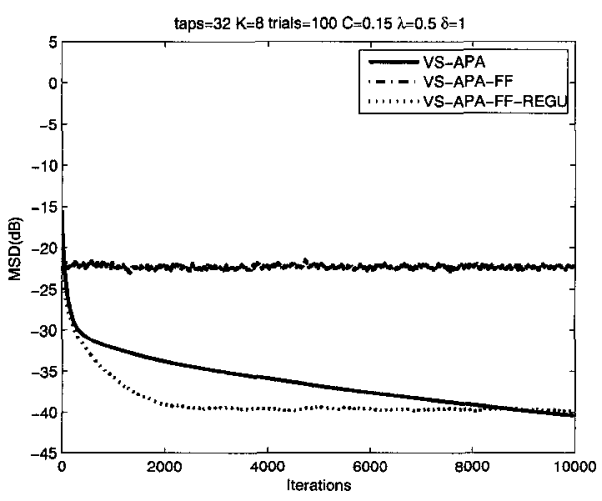

(a)

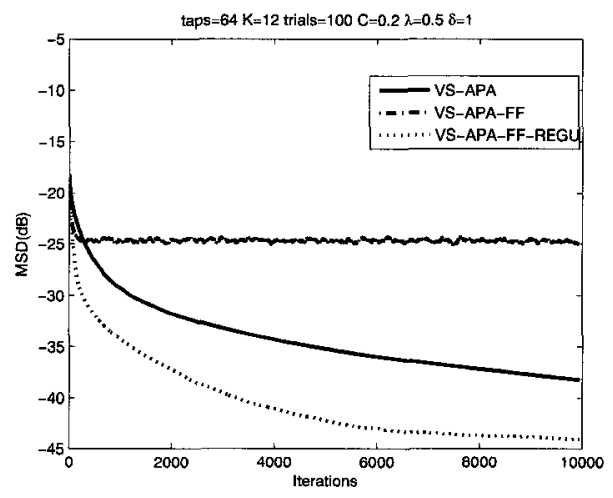

(b)

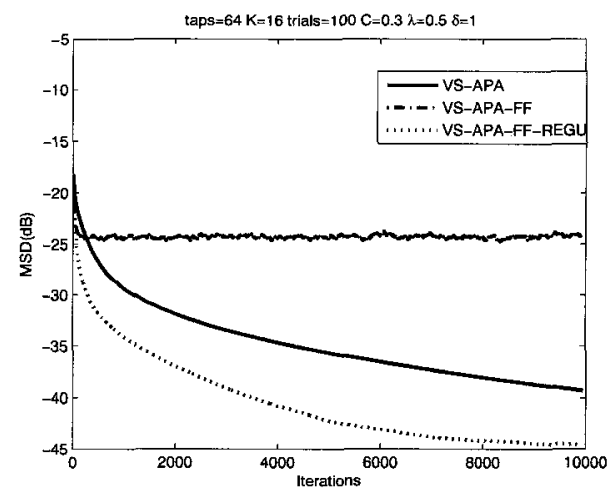

(c)

Figure A.5: Comparisons among VS-APA, VS-APA-FF, and VS-APA-FF-REGU, G1 colorization. $\lambda=0.5$. (a) $\mathrm{K}=8$, taps $=32, \mathrm{C}=0.15$; (b) $\mathrm{K}=12$, taps $=64, \mathrm{C}=0.2 ;$ (c) $\mathrm{K}=16$, taps $=64, \mathrm{C}=0.3$

\section{A.7 Conclusions}

This appendix presents an upgraded variable step size APA algorithm, $V S-A P A-F F$, and its regularized version $V S-A P A-F F-R E G U$. With a projection matrix processed with a forgetting factor, $V S-A P A-F F$ obtains upgraded convergence performance in terms of higher convergence rate and lower misadjustment. However, due to the singularity becomes worse after been weighted, $V S-A P A-F F$ is incapable of some cases, such as the APA window size $K$ is large and a small forgetting factor $\lambda$ is used. The Tikhonov regularization is used to overcome the deteriorated singularity problem of the processed input matrix. The regularized algorithm is more stable and converges better than previous algorithms. 


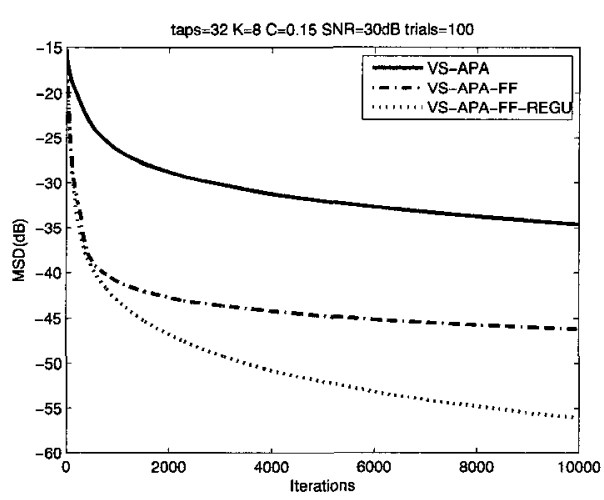

(a)

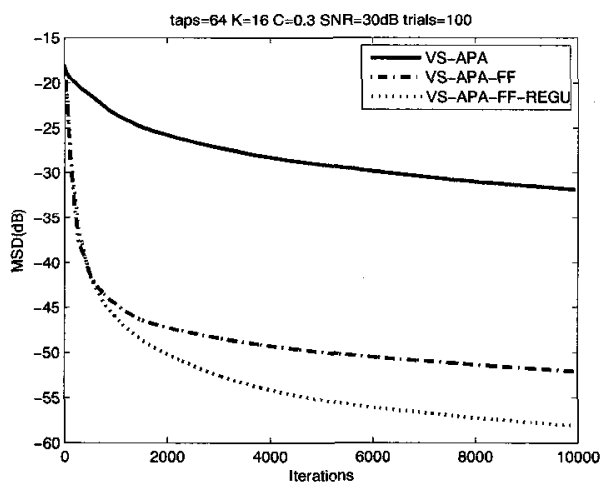

(c)

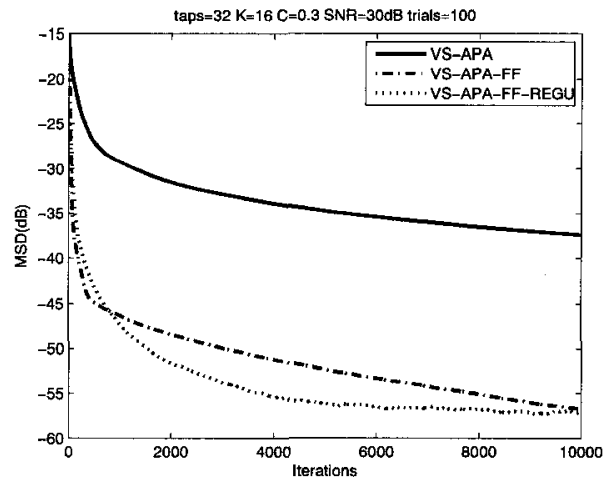

(b)

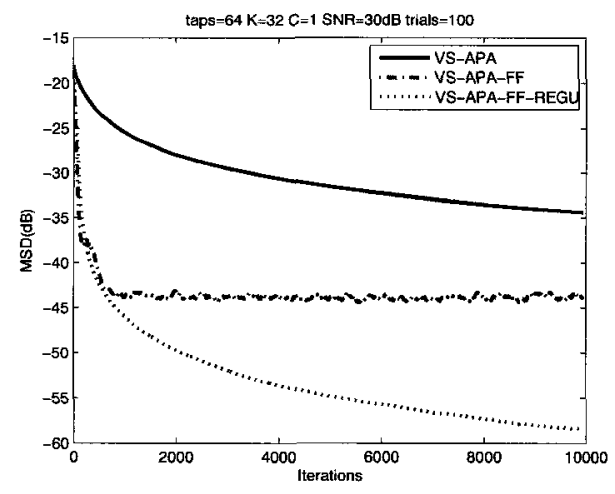

(d)

Figure A.6: Comparisons between algorithms: VS-APA, VS-APA-FF and VS-APA-FF-REGU. G2 colorization, $\lambda=0.9, \delta=1, \mathrm{SNR}=30 \mathrm{~dB}$. (a) $\mathrm{K}=8$, taps $=32, \mathrm{C}=0.15$; (b) $\mathrm{K}=16$, taps $=32$, $\mathrm{C}=0.3 ;$ (c) $\mathrm{K}=16$, taps $=64, \mathrm{C}=0.3$; (d) $\mathrm{K}=32$, taps $=64, \mathrm{C}=1$; 


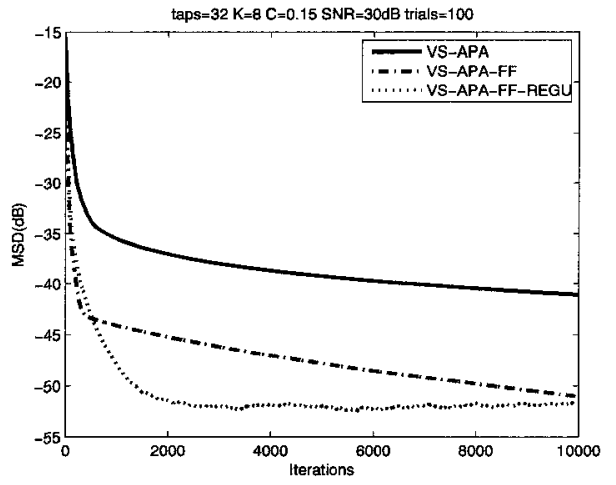

(a)

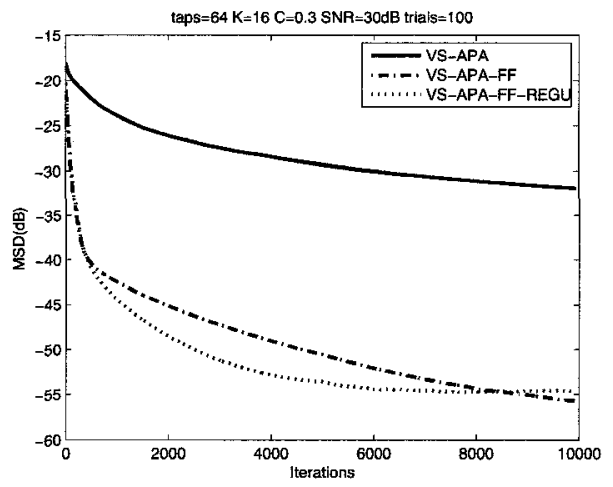

(c)

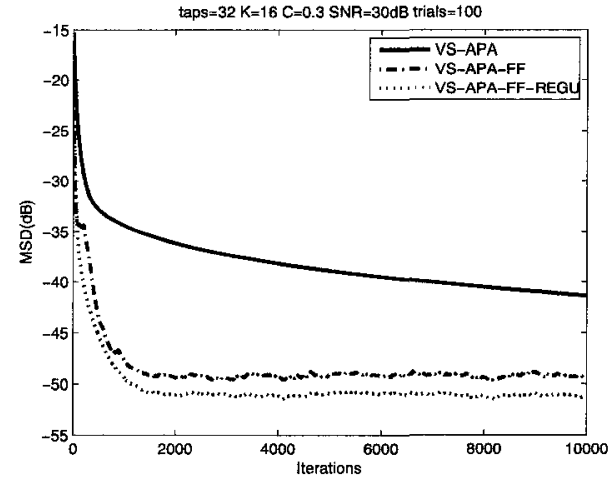

(b)

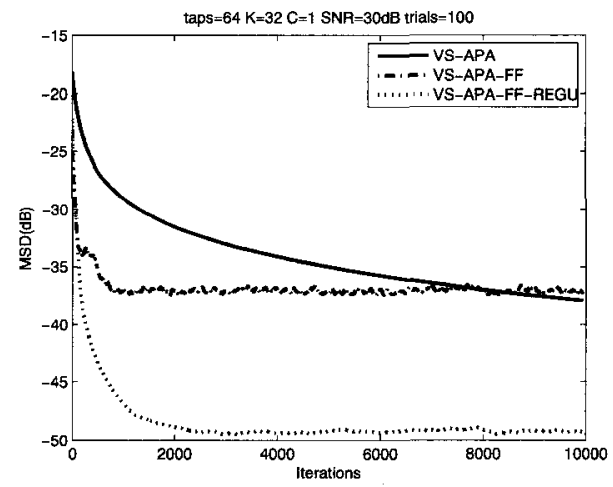

(d)

Figure A.7: Comparisons between algorithms: VS-APA, VS-APA-FF and VS-APA-FF-REGU. G1 colorization, $\lambda=0.9, \delta=1, \mathrm{SNR}=30 \mathrm{~dB}$. (a) $\mathrm{K}=8$, taps $=32, \mathrm{C}=0.15$; (b) $\mathrm{K}=16$, taps $=32$, $\mathrm{C}=0.3$; (c) $\mathrm{K}=16$, taps $=64, \mathrm{C}=0.3 ;$ (d) $\mathrm{K}=32$, taps $=64, \mathrm{C}=1$; 


\section{Appendix B}

\section{Formulation of $\ell_{1}$ Norms Regularization}

This appendix includes derivations that are not included in Chapter 6, Section 6.3.3, for conciseness. B.1 calculate the exponent value of the D matrix; B.2 mathematically derives the iterative formation; B.3 provide a data form method to reduce size of reconstruction matrix during iterations.

\section{B.1 Exponent on D}

We want a $p_{n}$-norm. If the exponent on the $\mathbf{D}$ matrix is $p$, then we have

$$
2+2 p=p_{n}
$$

which yeilds $1+p=p_{x} / 2$, and $p=p_{x} / 2-1$.

\section{B.2 Inverse formulation}

$$
\epsilon=(\mathbf{y}-\mathbf{J} \mathbf{x})^{t} \mathbf{W}(\mathbf{y}-\mathbf{J} \mathbf{x})+\left(\mathbf{x}-\mathbf{x}_{0}\right)^{t} \mathbf{R}\left(\mathbf{x}-\mathbf{x}_{0}\right)
$$


Minimum at derivative $d \epsilon / d \mathbf{x}=0$

$$
\begin{aligned}
0 & =-2 \mathbf{J}^{t} \mathbf{W}(\mathbf{y}-\mathbf{J} \mathbf{x})+2 \mathbf{R}\left(\mathbf{x}-\mathbf{x}_{0}\right) \\
\mathbf{J}^{t} \mathbf{W} \mathbf{J} \mathbf{x}+\mathbf{R} \mathbf{x} & =\mathbf{J}^{t} \mathbf{W} \mathbf{y}+\mathbf{R} \mathbf{x}_{0} \\
& =\mathbf{J}^{t} \mathbf{W}\left(\mathbf{y}-\mathbf{J} \mathbf{x}_{0}+\mathbf{J} \mathbf{x}_{0}\right)+\mathbf{R} \mathbf{x}_{0} \\
& =\mathbf{J}^{t} \mathbf{W}\left(\mathbf{y}-\mathbf{J} \mathbf{x}_{0}\right)+\mathbf{J}^{t} \mathbf{W} \mathbf{J} \mathbf{x}_{0}+\mathbf{R} \mathbf{x}_{0} \\
\left(\mathbf{J}^{t} \mathbf{W J}+\mathbf{R}\right) \mathbf{x} & =\mathbf{J}^{t} \mathbf{W}\left(\mathbf{y}-\mathbf{J} \mathbf{x}_{0}\right)+\left(\mathbf{J}^{t} \mathbf{W} \mathbf{J}+\mathbf{R}\right) \mathbf{x}_{0} \\
\left(\mathbf{J}^{t} \mathbf{W J}+\mathbf{R}\right)\left(\mathbf{x}-\mathbf{x}_{0}\right) & =\mathbf{J}^{t} \mathbf{W}\left(\mathbf{y}-\mathbf{J} \mathbf{x}_{0}\right) \\
\mathbf{x}-\mathbf{x}_{0} & =\left(\mathbf{J}^{t} \mathbf{W} \mathbf{J}+\mathbf{R}\right)^{-1} \mathbf{J}^{t} \mathbf{W}\left(\mathbf{y}-\mathbf{J} \mathbf{x}_{0}\right) \\
\mathbf{x} & =\mathbf{x}_{0}+\left(\mathbf{J}^{t} \mathbf{W J}+\mathbf{R}\right)^{-1} \mathbf{J}^{t} \mathbf{W}\left(\mathbf{y}-\mathbf{J} \mathbf{x}_{0}\right)
\end{aligned}
$$

\section{B.3 Data formulation for inverse}

The previous equation suggests an iterative update formula where $\mathbf{x}^{(k+1)}$ is calculated from $\mathbf{x}^{(k)}$ by:

$$
\mathbf{x}^{(k+1)}=\mathbf{x}^{(k)}+\left(\mathbf{J}^{t} \mathbf{W} \mathbf{J}+\mathbf{R}\right)^{-1} \mathbf{J}^{t} \mathbf{W}\left(\mathbf{y}-\mathbf{J} \mathbf{x}^{(k)}\right)
$$

Start with the matrix $\left(\mathbf{J}^{t} \mathbf{W} \mathbf{J}+\mathbf{R}\right)^{-1} \mathbf{J}^{t} \mathbf{W}$. Multiply by the identity matrix, as $\left(\mathbf{J R}^{-1} \mathbf{J}^{t}+\right.$ $\left.\mathbf{W}^{-1}\right)\left(\mathbf{J R}^{-1} \mathbf{J}^{t}+\mathbf{W}^{-1}\right)^{-1}$ to get

$$
\begin{array}{r}
\left(\mathbf{J}^{t} \mathbf{W} \mathbf{J}+\mathbf{R}\right)^{-1} \mathbf{J}^{t} \mathbf{W}\left(\mathbf{J} \mathbf{R}^{-1} \mathbf{J}^{t}+\mathbf{W}^{-1}\right)\left(\mathbf{J R}^{-1} \mathbf{J}^{t}+\mathbf{W}^{-1}\right)^{-1} \\
\left(\mathbf{J}^{t} \mathbf{W} \mathbf{J}+\mathbf{R}\right)^{-1}\left(\mathbf{J}^{t} \mathbf{W} \mathbf{J} \mathbf{R}^{-1} \mathbf{J}^{t}+\mathbf{J}^{t} \mathbf{W} \mathbf{W}^{-1}\right)\left(\mathbf{J} \mathbf{R}^{-1} \mathbf{J}^{t}+\mathbf{W}^{-1}\right)^{-1} \\
\left(\mathbf{J}^{t} \mathbf{W} \mathbf{J}+\mathbf{R}\right)^{-1}\left(\mathbf{J}^{t} \mathbf{W} \mathbf{J} \mathbf{R}^{-1} \mathbf{J}^{t}+\mathbf{J}^{t}\right)\left(\mathbf{J R}^{-1} \mathbf{J}^{t}+\mathbf{W}^{-1}\right)^{-1} \\
\left(\mathbf{J}^{t} \mathbf{W} \mathbf{J}+\mathbf{R}\right)^{-1}\left(\mathbf{J}^{t} \mathbf{W} \mathbf{J} \mathbf{R}^{-1}+\mathbf{I}\right) \mathbf{J}^{t}\left(\mathbf{J R}^{-1} \mathbf{J}^{t}+\mathbf{W}^{-1}\right)^{-1} \\
\left(\mathbf{J}^{t} \mathbf{W} \mathbf{J}+\mathbf{R}\right)^{-1}\left(\mathbf{J}^{t} \mathbf{W} \mathbf{J} \mathbf{R}^{-1}+\mathbf{R} \mathbf{R}^{-1}\right) \mathbf{J}^{t}\left(\mathbf{J R}^{-1} \mathbf{J}^{t}+\mathbf{W}^{-1}\right)^{-1} \\
\left(\mathbf{J}^{t} \mathbf{W} \mathbf{J}+\mathbf{R}\right)^{-1}\left(\mathbf{J}^{t} \mathbf{W} \mathbf{J}+\mathbf{R}\right) \mathbf{R}^{-1} \mathbf{J}^{t}\left(\mathbf{J R}^{-1} \mathbf{J}^{t}+\mathbf{W}^{-1}\right)^{-1} \\
\mathbf{R}^{-1} \mathbf{J}^{t}\left(\mathbf{J} \mathbf{R}^{-1} \mathbf{J}^{t}+\mathbf{W}^{-1}\right)^{-1}
\end{array}
$$

Thus, eqn B.11 becomes

$$
\mathbf{x}^{(k+1)}=\mathbf{x}^{(k)}+\mathbf{R}^{-1} \mathbf{J}^{t}\left(\mathbf{J R}^{-1} \mathbf{J}^{t}+\mathbf{W}^{-1}\right)^{-1}\left(\mathbf{y}-\mathbf{J} \mathbf{x}^{(k)}\right)
$$




\section{Bibliography}

A. Adler. Measurement of Pulmonary Function with Electrical Impedance Tomography. PhD thesis, Institut de Génie Biomédical Université de Montréal, 1995.

A. Adler and R. Guardo. Electrical impedance tomography: regularized imaging and contrast detection. IEEE Trans. Med. Imaging, 15:170-179, 1996a.

A. Adler and W.R.B. Lionheart. Uses and abuses of EIDORS: An extensible software base for EIT. Physiol. Meas., 27:S25-S42, 2006.

A. Adler, R. Guardo, and Y. Berthiaume. Impedance imaging of lung ventilation: Do we need to account for chest expansion? IEEE Trans. Biomed. Eng., 43(4):414-420, 1996b.

A. Adler, T. Dai, and W.R.B. Lionheart. Temporal image reconstruction in electrical impedance tomography. Physiol. Meas., 28:S1-S11, 2007.

G. Alessandrini and L. Rondi. Stable determination of a crack in a planar inhomogeneous conductor. SIAM J. Math. Anal., 30:326-340, 1998.

J.M. Alison and R.J. Sheppard. Dielectric properties of human blood at microwave frequencies. Phys. Med. Biol., 38:971-978, 1993.

Y. Asfaw and A. Adler. Automatic detection of detached and erroneous electrodes in electrical impedance tomography. Physiol. Meas., 26:S175-S183, 2005.

R.C. Aster, B. Borchers, and C.H. Thurber. Parameter Estimation and Inverse Problems. Elsevier Academic Press, 1 edition, 2005.

S. Babaeizadeh and D.H. Brooks. Effects of electrode location error on boundary element impedance tomography solutions: CR bounds and simulation results. 3rd IEEE ISBI: Nano to Macro, pages 1080-1083, 2006. 
S. Babaeizadeh and D.H. Brooks. Electrical impedance tomography for piecewise constant domains using boundary element shape-based inverse solutions. IEEE Trans. on Medical Imaging, 26(5):637-647, 2007.

C.C. Bacrie, Y. Goussard, and R. Guardo. Regularized reconstruction in electrical impedance tomography using a variance uniformization constraint. IEEE Trans.Med.Imag., 16(5):562-571, 1997.

D.C. Barber. Electrical impedance tomography: methods, history and applications, chapter Chapter 11, EIT: The view from Sheffield. IOP, Bristol and Philadelphia, 2005.

D.C. Barber and B.H. Brown. Applied potential tomography (review article). J. Phys. E:Sci. Instrum., 17:723-733, 1984.

D.C. Barber and B.H. Brown. Information Processing in Medical Imaging, chapter Recent development in applied potential tomography, pages 106-121. The hague: Nijhoff, 1986.

D.C. Barber and B.H. Brown. Errors in reconstruction of resistivity images using a linear reconstruction technique. Clin. Phys. Physiol. Meas., 9(suppl.A):101-104, 1988.

M.S. Beck, T. Dyakowski, and R.A. Willliams. Process tomography-the state of the art. Trans.Inst. Meas. And Control, 20(4):163-177, 1998.

A. Bollmann, N.K. Kanuru, K.K. McTeague, P.F. Walter, D.B. DeLurgio, and J.J. Langberg. Frequency analysis of human atrial fibrillation using the surface electrocardiogram and its response to ibutilide. Am J Cardiol., 81(12):1439-1445, 1998.

K.G. Boone and D.S. Holder. Effect of skin impedance on image quality and variability in electrical impedance tomography: a model study. Med. Biol. Eng. Comput., 34(5): $351-354,1996$.

A. Borsic, B.M. Graham, A. Adler, and W.R.B. Lionheart. Total variation regularization in electrical impedance tomography. submitted, 2007.

J.R. Bourne, J.P. Morucci, M.E. Valentinuzzi, B. Rigaud, C.J. Felice, N. Chauveau, and P.M. Marsili. Bioelectrical impedance techniques in medicine. Critical Reviews in Biomedical Engineering, 24(4-6), 1996.

B.H. Brown and A.D. Seagar. The sheffield data collection system. Physiol. Meas., 8(A): $91-98,1987$. 
B.H. Brown, D.C. Barber, A.H. Morice, and A.D. Leathard. Cardiac and respiratory related electrical impedance changes in the human thoraxs. IEEE Trans. Biomed. Eng., 41:729-734, 1994.

J.H. Campbell, N.D. Harris, F. Zhang, B.H. Brown, and A.H. Morice. Clinical applications of electrical impedance tomography in the monitoring of changes in intrathoracic fluid volumes. Physiol. Meas., 15:A217-A222, 1994.

O. Casas, R. Bragos, R.J. Riu, J. Rosell, M. Tresanchez, M. Warren, A. Rodriguez-Sinovas, A. Carrena, and J. Cinca. in vivo and in situ ischemic tissue characterization using electrical impedance spectroscopy. Ann. NY. Acad. Sci., 873:51-58, 1999.

M. Cetin and W.C. Karl. Feature-enhanced synthetic aperture radar image formation based on nonquadratic regularization. IEEE Trans. Image Processing, 10(4):623-631, 2001.

T.F. Chan, G.H. Golub, and P. Mulet. A nonlinear primal-dual method for total variationbased image restoration. SIAM Journal on Scientific Computing, 20(6):1964-1977, 1999.

M. Cheney, D. Isaacson, J.C. Newell, S. Simske, and J.C. Goble. NOSER: an algorithm for solving the inverse conductivity problem. Int.J.Imaging Syst.Technol., 2:66-75, 1990.

M. Cheney, D. Isaacson, E.J. Somersalo, and E.L. Isaacson. Layer-stripping reconstruction algorithm for impedance imaging. In Proc. Ann. Int. Conf. IEEE Eng. Med. Biol. Sci., volume 14, pages 1694-1695, Paris, France, 1992.

K.S. Cole. Permeability and impermeability of cell membranes for ions. Cold Sring Harbor Symp. Quant. Biol., pages 110-122, 1940.

K.S. Cole and R.H. Cole. Dispersion and absorption in dielectrics. I. alternating current field. Journal of Chemical Physics, 1:341-351, 1941.

R. Kress D. Colton. Inverse Acoustic and Electromagnetic Scattering Theory. Springer, Berlin, 2 edition, 1998.

J.E. da Silva, J.P. de Sa, and J. Jossinet. Classification of breast tissue by electrical impedance spectroscopy. Med. Biol. Eng. Comput., 38(1):26-30, 2000.

T. Dai and A. Adler. Blood impedance characterization from pulsatile measurements. In IEEE Electrical and Computer Engineering, Canadian Conference (CCECE), pages 983-986, 2006. 
T. Dai and A. Adler. Electrical impedance tomography reconstruction using 11 norm on data and image terms. In 30th Annual International Conference of the IEEE EMBC, Vancouver, Canada, August 2008c.

T. Dai, M. Soleimani, and A. Adler. Four-dimensional regularization for electrical impedance tomography imaging. In IFMBE Proceedings, VIII Conference on Electrical Impedance Tomography, volume 17, page 408, Graz, Austria., Aug29 Sept2 2007.

T. Dai, C. Gómez-Laberge, and A. Adler. Reconstruction of conductivity changes and electrode movements based on EIT temporal sequences. Physiol. Meas., 29:S77-S88, 2008a.

T. Dai, M. Soleimani, and A. Adler. EIT image reconstruction with four dimensional regularization. Medical and Biological Engineering and Computing, In Press, 2008b.

H. Dehgani, N. Soni, R. Halter, A. Hartov, and K.D. Paulsen. Excitation patterns in three-dimensional electrical impedance tomography. Physiol. Meas., 26:185-197, 2005.

F. Dickin and M. Wang. Electrical resistance tomography for process tomography. Meas. Sci. Tech., 7:247-260, 1996.

A.M. Dijkstra, B.H. Brown, A.D. Leathard, N.D. Harris, D.C. Barber, and D.L. Edbrooke. Clinical applications of electrical impedance tomography. J. Med. Eng. Technol., 17(3): $8998,1993$.

K.A. Dines and R.J. Lytle. Analysis of electrical conductivity imaging. Geophysics, 46: $1025-1036,1981$.

T.J. Ebert, J.J. Smith, J.A. Barney, D.C. Merrill, and G.K. Smith. The use of thoracic impedance for determining thoracic blood volume changes in man. Aviat. Space Environ. Med., 57:49-53, 1986.

J.F. Edd, A. Ivorra, L. Horowitz, and B. Rubinsky. Imaging cryosurgery with EIT: tracking the ice front and post-thaw tissue viability. Physiol. Meas., 29:899-912, 2008.

E.C. Eichenwald and A.R. Stark. High frequency ventilation: Current status. Pediatric Review, 20:127-133, 1999.

B.M. Eyuboglu, B.H. Brown, and D.C. Barber. in vivo imaging of cardiac related impedance changes. IEEE Eng. Med. Biol. Mag., 8(1):39-45, 1989. 
L. Fahrmeir and G. Tutz. Multivariate Statistical Modeling Based on Generalized Linear Models. Springer-Verlag, New York, 1994.

K.R. Foster and H.P. Schawn. Dielectric properties of tissues and biological materials: a critical review. CRC Critical Reviews in Biomedical Engineering, 17(1):25-104, 1989.

I. Frerichs. Electrical impedance tomography (EIT) in applications related to lung and ventilation: a review of experimental and clinical activities. Physiol. Meas., 21:1-21, 2000 .

I. Frerichs, G. Hahn, and G. Hellige. Thoracic electrical impedance tomographic measurements during volume controlled ventilation-effects of tidal volume and positive endexpiratory pressure. IEEE Trans. Medical Imag., 18:768-778, 1999.

H. Fricke and S. Morse. The electric capacity of tumors of the breast. J. Cancer Res., 10: 340-376, 1926.

C. Gabriel, S. Gabriely, and E. Corthout. The dielectric properties of biological tissues: I. Literature survey. Phys. Med. Biol., 41(11):2231-2249, 1996a.

S. Gabriel, R.W. Lau, and C. Gabriel. The dielectric properties of biological tissues: II. Measurements in the frequency range $10 \mathrm{hz}$ to $20 \mathrm{GHz}$. Phys. Med. Biol., 41(11):2251$2269,1996 \mathrm{~b}$.

S. Gabriel, R.W. Lau, and C. Gabriel. The dielectric properties of biological tissues: III. parametric models for the dielectric spectrum of tissues. Phys. Med. Biol., 41(11):22712293, 1996c.

S.L. Gay and S. Tavathia. The fast affine projection algorithm. In ICASSP, pages 30233026, May 1995.

D.G. Gisser, D. Isaacson, and J.C. Newell. Current topics in impedance imaging. Clin. Phys. Physiol. Meas., 8(Suppl A):39-46, 1987.

C. Gómez-Laberge and A. Adler. Direct calculation of the electrode movement Jacobian for 3D EIT. In IFMBE Proceedings, VIII Conference on Electrical Impedance Tomography, volume 17, pages 364-367, Graz, Austria., Aug29 Sept2 2007.

C. Gómez-Laberge and A. Adler. Direct EIT jacobian calculations for conductivity change and electrode movement. Physiol. Meas., 29:S89-S99, 2008. 
B.M. Graham and A. Adler. Objective selection of hyperparameter for EIT. Physiol.Meas., 27:S65-S79, 2006.

B.M. Graham and A. Adler. Electrode placement configurations for 3d EIT. Physiol.Meas., 28:S29 S44, 2007.

S. Grimnes and O.G. Martinsen. Bioimpedance and Bioelectricity Basics. Academic, San Diego, CA, USA, 2000.

J. Hadamard. Sur les problémes aux dérivées partielles et leur signification physique. Princeton University Bulletin, pages 49-52, 1902.

R. Halter, A. Hartov, and K. Paulsen. Design and implementation of a high frequency electrical impedance tomography system. Physiol. Meas., 25:379-390, 2004.

R.J. Halter, A. Hartov, J.A. Heaney, K.D. Paulsen, and A.R. Schned. Electrical impedance spectroscopy of the human prostate. IEEE Trans on Biomedical Engineering, 54(7): $1321-1327,2007$.

R.J. Halter, A. Hartov, K.D. Paulsen, A. Schned, and J. Heaney. Genetic and least squares algorithms for estimating spectral eis parameters of prostatic tissues. Physiol. Meas., 29 (7):S111-S123, 2008a.

R.J. Halter, A. Schned, J. Heaney, A. Hartov, S. Schutz, and K.D. Paulsen. Electrical impedance spectroscopy of benign and malignant prostatic tissues. J Urol., 179(4): 1580-1586, 2008b.

K.E. Hammermeister, R.C. Brooks, and J.R. Warbasse. The rate of change of left ventricular volume in man: I. Validation and peak systolic ejection rate in health and disease. Circulation, 49:729 738, 1974.

J. Harms, A. Schneider, M. Baumgarter, J. Henke, and R. Busch. Diagnosing acute liver graft rejection: experimental application of an implantable telemetric impedance device in native and transplanted porcine livers. Biosensors and Biolectronics, 16:169-177, 2001.

N.D. Harris, A.J. Suggett, D.C. Barber, and B.H. Brown. Applied potential tomography: a new technique for monitoring pulmonary function. Clin. Phys. Physiol. Meas., 9:79-85, 1988. 
N.D. Harris, B.H. Brown, and D.C. Barber. Continuous monitoring of lung ventilation with electrical impedance tomography. Med. Biol. Soc., pages 1754-1755, 1992.

R.W. Harris, D. Chabries, and F. Bishop. A variable step (vs) adaptive filter algorithm. IEEE Trans. Acoust. Speech Signal Process, 34(2):309-316, 1986.

S. Haykin. Adaptive Filter Theory. Upper Saddle River, NJ: Prentice-Hall, 4 edition, 2002.

S. Haykin and B.Widrow. Least-Mean-Square Adaptive Filters. Wiley interscience, 2003.

R.P. Henderson and J.G. Webster. An impedance camera for spatially specific measurements of the thorax. IEEE Trans. Biomed. Eng., 25:250-254, 1978.

B.J. Hoenders. Inverse Problems in Optics. Springer-Verlag, Berlin,German, 4 edition, 1978.

B.J. Hoenders. Existence of invisible nonscattering objects and nonradiating sources. $J$. Opt. Soc. Am. A, 14(1):262-266, 1997.

D. Holder. Electrical impedance tomography: methods, history and applications, chapter Appendix B: Introduction to biomedical electrical impedance tomography. IOP, Bristol and Philadelphia, 2005.

D.S. Holder. Feasibility of developing a method of imaging neuronal activity in the human brain: a theoretical review. Med. Biol. Eng comput., 25:2-11, 1987.

D.S. Holder. Electrical impedance tomography of brain function. Brain Topography, 5: 87-93, 1992.

A. Hunt, J.D. Pendleton, and R.B. White. A novel tomographic flow analysis system. In 3rd World Congress on Industrial Process Tomography, Banff, Canada, 2-5 September 2003.

K. Ikeda. Convergence analysis of block orthogonal projection and affine projection algorithms. Signal Processing, 82:491-496, 2002.

D. Isaacson. Distinguishability of conductivities by electric-current computed-tomography. IEEE Tran. Med. Imaging, 5:91-95, 1986. 
D. Isaacson, J.L. Mueller, J.C. Newell, and S. Siltanen. Reconstructions of chest phantoms by the d-bar method for electrical impedance tomography. IEEE Tran. Med. Imaging, 23(7):821-828, 2004.

D. Isaacson, J.L. Mueller, J.C. Newell, and S. Siltanen. Imaging cardiac activity by the d-bar method for electrical impedance tomography. Physiol. Meas., 27(5):S43-S50, 2006.

A. Ivorra. Bioimpedance Monitoring for physicians: an overview. Biomedical Applications Group, Barcelona, 2002.

K.V. Jenderka and E. Gersing. Comparison of the impedance and ultrasound spectroscopy in investigations of ischaemia caused alterations in organ tissue. In 18th Annual International Conference of the IEEE Engineering in Medicine and Biology Society, pages 855-856, Amsterdam, 1996.

J. Jossinet. Variability of impedivity in normal and pathological breast tissue. Med. Biol. Eng. Comput., 34:346-350, 1996.

J. Jossinet and M. Schmitt. A review of parameters for the bioelectrical characterization of breast tissue. Annals of the New York Academy of Sciences, 873:30-41, 1999.

J.P. Kaipio, V. Kolehmainen, M. Vauhkonen, and E. Somersalo. Construction of nonstandard smothness priors. Inverse Problems, 15:713-729, 1999.

H. Kanai, M. Haeno, and K. Sakamoto. Electrical measurement of fluid distribution in legs and arms. Med. Progress through Techno., 12:159-170, 1987.

T.E. Kerner. Electrical impedance tomography for breast imaging. $\mathrm{PhD}$ thesis, Dartmouth College, 2001.

T.E. Kerner, K.D. Paulsen, A. Hartov, S.K. Soho, and S.P. Poplack. Electrical impedance spectroscopy of the breast: clinical imaging results in 26 subjects. IEEE Trans. Med. Imaing, 21(6):638-645, 2002.

A. Keshtkar, A. Keshtkar, and R.H. Smallwood. Electrical impedance spectroscopy and the diagnosis of bladder pathology. Physiol. Meas., 27(7):585-596, 2006.

M. Khan and S.K. Guha. Prediction of electrical impedance parameters for the simulated leg segment of an aircraft pilot under G-stress. Aviation, Space, and Environmental Medicine, 73(6):558-564, 2002. 
B.S. Kim, K.Y. Kim, T.J. Kao, J.C. Newell, D. Isaacson, and G.J. Saulnier. Dynamic electrical impedance imaging of a chest phantom using the kalman filter. Physiol.Meas., 27:S81-S91, 2006.

B.S. Kim, D. Isaacson, H. Xia, T.J. Kao, J.C. Newell, and G.J. Saulnier. A method for analyzing electrical impedance spectroscopy data from breast cancer patients. Physiol.Meas., 28(7):S237-S246, 2007.

K.Y. Kim, B.S. Kim, M.C. Kim, and S. Kim. Dynamic inverse obstacle problems with electrical impedance tomography. Mathematics and Computers in Simulation, 66:399408, 2004.

B.U. Kohler, C. Hennig, and R. Orglmeister. The principles of software QRS detection. IEEE Eng Med Biol Magazine, 21:42-57, 2002.

P.W.A. Kunst, A. Vonk-Noordegraaf, O.S. Hoekstra, P.E. Postmus, and P.M.J.M. de Vries. Ventilation and perfusion imaging by electrical impedance tomography: A comparison with radionuclide scanning. Physiol. Meas., 19:481-490, 1998.

A.L. Lafargue, L.B. Cabrales, and R.M. Larramendi. Bioelectrical parameters of the whole human body obtained through bioelectrical impedance analysis. Bioelectromagnetics, 23: $450-454,2002$.

H.G. Linhart, E. Gersing, M. Schafer, and M.M. Gebhard. The electrical impedance of ischemic heart at low frequencies. In IX International Conference On Bio-Impedance, pages 207-210, Heidelberg, Germany, September 26-30 1995.

W. Lionheart, N. Polydorides, and A. Borsic. Electrical impedance tomography: methods, history and applications, chapter The Reconstruction Problem. IOP, Bristol and Philadelphia, 2005.

W.R. Lionheart. EIT reconstruction algorithms: pitfalls, challenges and recent developments. Physiol. Meas., 25:125-142, 2004.

M.H. Loke and R.D. Barker. Practical techniques for 3d resistivity surveys and data inversion. Geophysical Prospecting, 44:499-523, 1996.

X. Ma, A.J. Peyton, R. Binns, and S.R. Higson. Imaging the flow profile of molten steel through a submerged pouring nozzle. In 3rd World Congress on Industrial Process Tomography, pages 736-742, Banff, Canada, 2-5 September 2003. 
A. Mader, H. Puder, and G.U. Schmidt. Step-size control for acoustic echo cancellation filters-an overview. Signal Process., 80:1697-1719, 2000.

F.J. McArdle, A.J. Suggett, B.H. Brown, and D.C. Barber. An assessment of dynamic images by applied potential tomography for monitoring pulmonary perfusion. Clin. Phys. Physiol. Meas., 9:87-91, 1988.

P. Metherall, D.C. Barber, R.H. Smallwood, and B.H. Brown. Three dimensional electrical impedance tomography. Nature, 380:509-512, 1996.

J.J. Moré. The levenberg-marquardt algorithm: implementation and theory. In Numerical Analysis, pages 105-116. Springer Verlag, 1977.

T. Morimoto, Y. Kinouchi, T. Iritani, S. Kimura, Y. Konishi, N. Mitsuyama, K. Komaki, and Y. Monden. Measurement of the electrical bio-impedance of breast tumors. Eur. Surg. Res., 22(2):86-92, 1990.

J.P. Morucci and P.M. Marsili. Bioelectrical impedance techniques in medicine. part iii: Impedance imaging. second section: reconstruction algorithms. Crit. Rev. Biomed. Eng., 24:599-654, 1996.

J. Nagumo and A. Noda. A learning method ofr system identification. IEEE Trans. Automat. Control, 12:282-287, 1967.

J. Nyboer. Electrical Impedance Plethymography. Springfield, Illinois, 2nd edition, 1970.

P.A. Olsen and A.R. Gopinath. Modeling inverse covariance matrices by basis expansion. IEEE Trans Speech Audio Processing, 12:37-46, 2004.

S.J. Orfanidis. Optimum Signal Processing, An Introduction. MacMillan, New York, 1985.

J.A. Ormiston, P.M. Shah, C. Tei, and M. Wong. Size and motion of the mitral valve annulus in man. I. A two-dimensional echocardiographic method and findings in normal subjects. Circulation, 64:113-120, 1981.

D.M. Otten and B. Rubinsky. Cryosurgical monitoring using bioimpedance measurementsafeasibility study for electrical impedance tomography. IEEE Tran. Biom. Eng., 47(10): $1376-1381,2000$. 
D.M. Otten and B. Rubinsky. Front-tracking image reconstruction algorithm for EITmonitored cryosurgery using the boundary element method. Physiol. Meas., 26(4):503$516,2005$.

K. Ozeki and T. Umeda. An adaptive filtering algorithm using an orthogonal projection to an affine subspace and its properties. Electron. Commun. Jpn., 67a(5):19-27, 1984.

N. Polydorides and W.R.B Lionheart. A Matlab toolkit for three-dimensional electrical impedance tomography: A contribution to the Electrical Impedance and Diffuse Optical Reconstruction Software project. Meas. Sci. Technol., 13:1871-1883, 2002.

L.R. Price. Electrical impedance computed tomography (ICT): a new CT imaging technique. IEEE trans. Nucl.Sci., 26:2736-2739, 1979.

B. Rigaud, L. Hamzaoui, M.R. Frikha, N. Chauveau, and J.P. Morucci. in vitro tissue characterization and modelling using electrical impedance measurements in the $100 \mathrm{~Hz}-$ $10 \mathrm{MHz}$ frequency range. Physiol. Meas., 16:A15-A28, 1995.

S.G. Sankaran and A.A. Beex. Convergence behavior of affine projection algorithms. IEEE Trans. Signal Processing, 48(4):1086-1096, 2000.

J.A. Scales, A. Gersztenkorn, and S. Treitel. Fast lp solution of large, sparse, linear systems: Application to seismic travel time tomogrphy. Journal of Computational Physics, 75(2): 314-333, 1988.

H.P. Schawn. Electrical properties of tissue and cell suspensions. Adv. Biol. Med. Phys., 5:147-209, 1957.

J.L. Schepps and K.R. Foster. The UHF and microwave dielectric properties of normal and tumour tissues: variation in dielectric properties with tissue water content. Phys. Med. Biol., 25(6):1149-1159, 1980.

U. Schmitt and A.K. Louis. Efficient algorithms for the regularization of dynamic inverse problems: I. Theory. Inverse Problems, 18(3):645-658, 2002.

U. Schmitt, A.K. Louis, C. Wolters, and M. Vauhkonen. Efficient algorithms for the regularization of dynamic inverse problems: II. Applications. Inverse Problems, 18(3): $659-676,2002$. 
B. Scholz and R. Anderson. On electrical impedance scanning: Principles and simulations. Electromedica, 68:35-44, 2000.

A.D. Seagar. Probing with low frequency electric current. PhD thesis, Universit of Canterbury, 1983.

A. Seppanen, M. Vauhkonen adn P.J. Vauhkonen, E. Somersalo, and J.P. Kaipio. State estimation with fluid dynamical models in process tomography-an application with impedance tomography. Inverse Problems, 17:467-483, 2001.

H.C. Shin and A.H. Sayed. Transient behavior of affine projection algorithms. In ICASSP, volume 6, pages 353-356, 2003.

H.C. Shin, A.H. Sayed, and W.J. Song. Variable step-size NLMS and affine projection algorithms. IEEE Signal Proc. Letters, 11(2):132-135, 2004.

S.F. Siconolfi, R.J. Gretebeck, W.W. Wong, R.A. Pietrzyk, and S.S Suire. Assessing total body water from bioelectrical response spectroscopy. J. Appl. Physiol., 82:704-710, 1997.

S. Siltanen, J. Mueller, and D. Isaacson. An implementation of the reconstruction algorithm of a nachman for the $2 \mathrm{~d}$ inverse conductivity problem. Inverse Problems, 16(3):681-699, 2000 .

R.H. Smallwood, Y.F. Mangnall, and A.D. Leathard. Transport of gastric contents. Physiol. Meas., 15:175-188, 1994.

R.H. Smallwood, A. Keshtkar, B.A. Wilkinson, J.A. Lee, and F.C. Hamdy. Electrical impedance spectroscopy (eis) in the urinary bladder: the effect of inflammation and edema on identification of malignancy. IEEE Trans. Med. Imaging, 21(6):708-710, 2002.

A. Sola, L. Palacios, J. Lopez-Marti, A. Ivorra, N. Noguera, R. Gomez, R. Villa, J. Aguilo, and G. Hotter. Multiparametric monitoring of ischemia-reperfusion in rat kidney: effect of ischemic preconditioning. Transplantation, 74(6):744-749, 2003.

M. Soleimani, C. Gómez-Laberge, and A. Adler. Imaging of conductivity changes and electrode movement in EIT. Physiol. Meas., 27:S103-S113, 2006a.

M. Soleimani, A. Adler, T. Dai, and A.J. Peyton. Application of a single step temporal imaging of magnetic induction tomography for metal flow visualization. Insight, 50(1), 2008 . 
E. Somersalo, M. Cheney, D. Isaacson, and E. Isaacson. Layer stripping, a direct numerical method for impedance imaging. Inverse Problems, 7:899-926, 1991.

A.J. Surowiec, S.S. Stuchly, J.R. Barr, and A. Swamp. Dielectric properties of breast carcinoma and the surrounding tissues. IEEE Trans. Biomed. Eng., 35(4):257-263, 1988.

A. Tamburrino and G. Rubinacci. A new non-iterative inversion method in electrical resistance tomography. Inverse Problems, 18(6):1809-1829, 2002.

B.J. Thomas, B.H. Cornish, and L.C. Ward. Bioelectrical impedance analysis for measurement of body fluid volumes: A review. J. Clin. Eng., 17:505-510, 1992.

A.T. Tidswell, A. Gibson, R.H. Bayford, and D.S. Holder. Electrical impedance tomography of human brain activity with a two-dimensional ring of scalp electrodes. Physiol. Meas., 22:167-175, 2001.

A.N. Tikhonov and V.Y. Arsenin. Solutions of Ill-Posed Problems. Winston, New York, 1977.

O.P. Tossavainen, M. Vauhkonen, L.M. Heikkinen, and T. Savolainen. Estimating shapes and freesurfaces with electrical impedance tomography. Measurem. Sci. Technol., 15: 1402-1411, 2004.

F.C. Trigo, R. Gonzalez-Lima, and M.B.P.A Amato. Electrical impedance tomography using the extended Kalman filter. IEEE Trans. Biomed. Eng., 51:72-81, 2004.

M.E. Valentinuzzi. Bioelectrical impedance techniques in medicine. part i: Bioimpedance measurement. first section:general concepts. Crit. Rev. in Biom. Eng., 24(4-6):223-255, 1996.

H.R. van Genderingen, A.J. van Vught, and J.R.C. Jansen. Regional lung volume during high-frequency oscillatory ventilation by electrical impedance tomography. Crit. Care Med., 32:787-794, 2004.

M. Vauhkonen, P.A. Karjalainen, and J.P. Kaipio. A Kalman filter approach to track fast impedance changes in electrical impedance tomography. IEEE Trans. Biomed. Eng., 45: 486-493, 1998a.

M. Vauhkonen, D. Vadàsz, P.A. Karjalainen, E. Somersalo, and J.P. Kaipio. Tikhonov regularization and prior information in electrical impedance tomography. IEEE Trans. Med. Imaging, 17:285-293, 1998b. 
P.J. Vauhkonen, M. Vauhkonen, T. Savolainen, and J.P. Kaipio. Three-dimensional electrical impedance tomography based on the complete electrode model. IEEE Trans. Med. Imaging, 46(9):1150-1160, 1999.

A. Vonk-Noordegraaf, A. Janse, J.T. Marcus, J.G. Bronzwaer, P.E. Postmus, T.J.C. Faes, and P.M. de Vries. Determination of stroke volume by means of electrical impedance tomography. Physiol. Meas., 21:285-293, 2000.

D.C. Walker, B.H. Brown, D.R. Hose, and R.H. Smallwood. Modelling the electrical impedivity of normal and premalignant cervical tissue. Electronics Letters, 36(19):1603$1604,2000$.

B. Widrow and S.D. Stearns. Adaptive Signal Processing. Englewood Cliffs, NJ: PrenticeHall, 1985.

A.J. Wilkinson, E.W. Randall, J.J. Cilliers, D.R. Durrett, T. Naidoo, and T. Long. A 1000-measurement frames/second ERT data capture system with real-time visualization. IEEE Sensors Journal, 5:300-307, 2005.

R.A. Williams and M.S. Beck. Process Tomography, Principles, Techniques and Applications. Butterworth-Heinemann Ltd, Oxford, 1995.

G.K. Wolf and J.H. Arnold. Noninvasive assessment of lung volume: Respiratory inductance plethysmography and electrical impedance tomography. Critical Care Medicine, 33:S163-S169, 2005.

T. Yamada, H. Hirose, Y. Mori, A. Onitsuka, M. Hayashi, S. Senga, N. Futamura, K. Sakamoto, T. Sago, H. Takgi, M. Yasumura, and H. Iwata. Dielectric spectrogram for evaluating ischemic microstructural changes of the liver in simple cold preservation. Surgery Today, 32:1058-1063, 2002.

K. Yamakoshi, H. Shimazu, T. Togawa, M. Fukuoka, and H. Ito. Non-invasive measurement of haematocrit by electrical admittance plethysmography technique. IEEE Trans. Biomed. Eng., 27:156-161, 1980.

T.J. Yorkey, J.G. Webster, and W.J. Tompkins. Comparing reconstruction algorithms for electrical impedance tomography. IEEE Trans. Biomed. Eng, 34:843-852, 1987.

F. Yu and M. Bouchard. Recursive least-squares algorithms with good numerical stability for multichannel active noise control. In ICASSP, volume 5, pages 3221-3224, 2001. 
J. Zhang and R.P. Patterson. EIT images of ventilation: what contributes to the resistivity changes? Physiol. Meas., 26:S81-S92, 2005.

T.X. Zhao, B. Jacobson, and T. Ribbe. Triple-frequency method for measuring blood impedance. Physiol. Meas., 14:145-156, 1993.

Y. Zou and Z. Guo. A review of electrical impedance techniques for breast cancer detection. Med. Eng. Phys., 25(2):79-90, 2003. 